\title{
$\alpha$-synuclein disrupts neuron network rhythmic activity when overexpressed in cultured neurons
}

\author{
Dissertation \\ for the award of the degree \\ "Doctor rerum naturalium" \\ of the Georg-August-Universität Göttingen
}

within the doctoral program Cellular and Molecular Physiology of the Brain of the Georg-August University School of Science (GAUSS)

submitted by

Kristian Leite

from Tallinn, Estonia

Göttingen 2020 


\section{Thesis Committee}

Dr. Sebastian Kügler (referee),

Department of Neurology, University Medical Center Göttingen

Prof. Dr. Thomas Dresbach ( $2^{\text {nd }}$ referee $)$,

Department of Anatomy and Embryology, University Medical Center Göttingen

Prof. Dr. Markus Zweckstetter,

Research Group "Protein structure determination using NMR",

Max Planck Institute for Biophysical Chemistry

\section{Members of the Examination Board}

Prof. Dr. Tiago Outeiro,

Department of Experimental Neurodegeneration, University Medical Center Göttingen

Prof. Dr. Michael Müller,

Department of Neurophysiology and Sensory Physiology, University Medical Center Göttingen

Prof. Dr. Alexander Flügel,

Institute for Neuroimmunology and Multiple Sclerosis Research, University Medical Center Göttingen

Date of thesis defense: February $9^{\text {th }}, 2021$

Printed and/or published with the support of the German Academic Exchange Service 


\section{Contents}

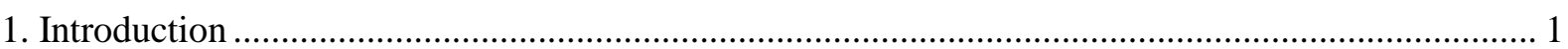

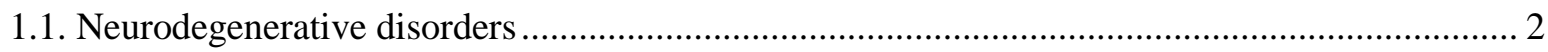

1.1.1. Parkinson's disease (PD) and Parkinson's disease dementia (PD-D) ................................... 2

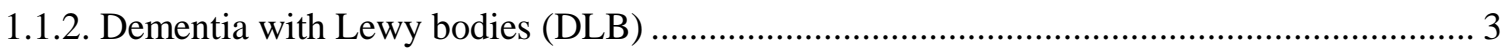

1.1.3. Alzheimer's disease (AD) and mild cognitive impairment (MCI) ...................................... 4

1.2. Neurodegenerative disorders and aggregating proteins.......................................................... 4

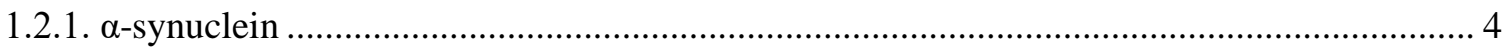

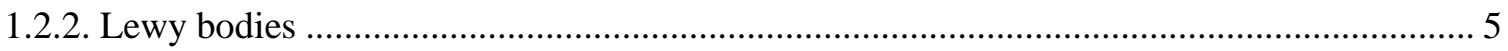

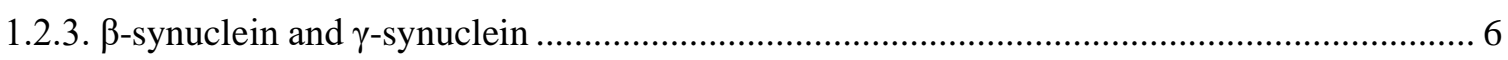

1.2.4. Microtubule-associated protein tau (tau protein) and neurofibrillary tangles ........................ 7

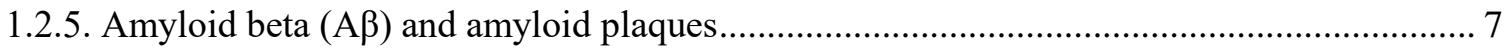

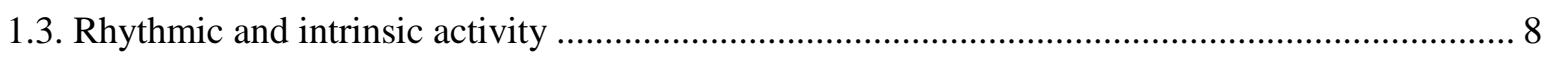

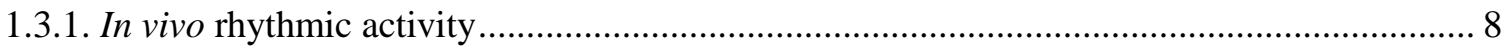

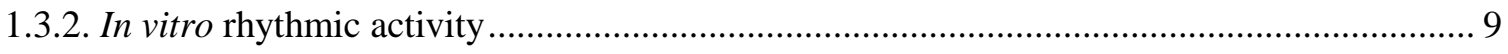

1.3.3. The role of $\alpha$-synuclein and tau protein in regulating rhythmic activity ............................ 10

1.3.4. Intrinsic activity and functional magnetic resonance imaging (fMRI) .............................. 11

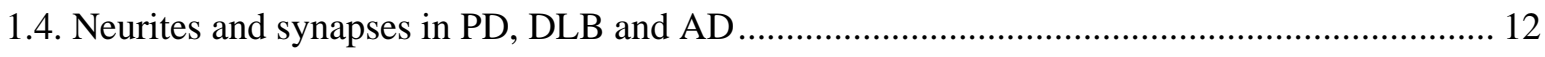

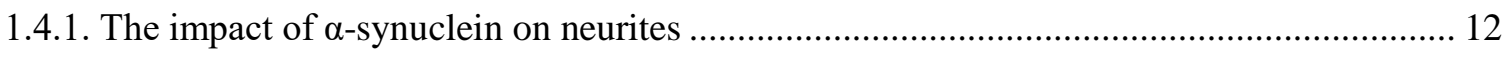

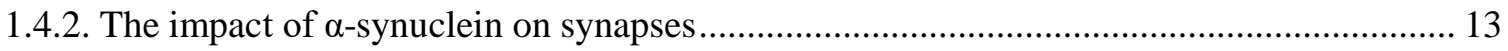

1.4.3. The impact of tau protein on neurites and synapses ....................................................... 14

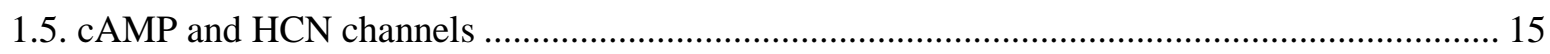

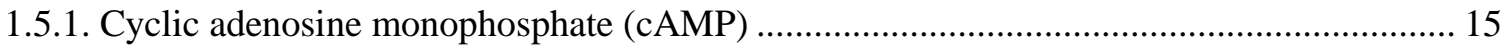

1.5.2. Hyperpolarization-activated cyclic nucleotide-gated channels (HCN channels)................ 16

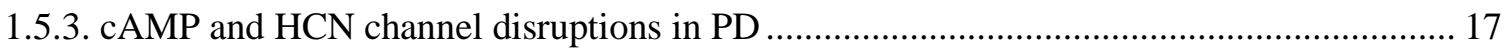

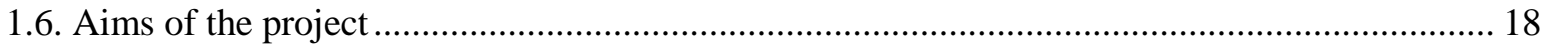

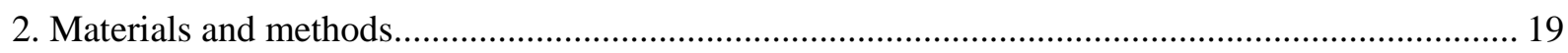


2.1. Experimental materials and their preparation.

2.1.1. Cultured neurons

2.1.2. Overexpressed proteins and sensors 20

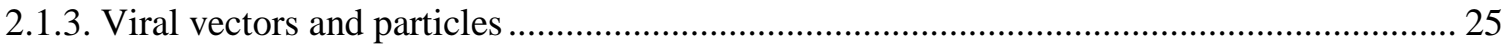

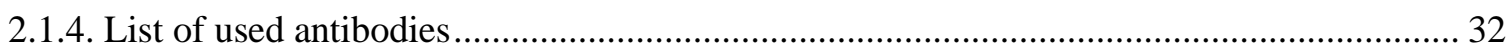

2.1.5. List of used macros, programs and calculation sheets.............................................. 33

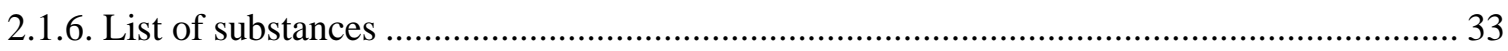

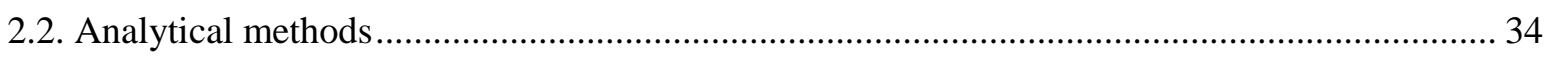

2.2.1. Recording of neuron network bursts .............................................................. 34

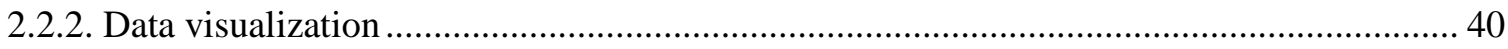

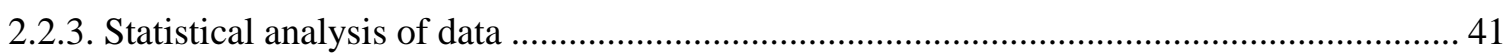

2.2.4. Quantification of protein amounts using a Western blot analysis ................................ 42

2.2.5. Studying the impact of extracellular $\alpha$-synuclein using cell culture supernatant exchange 46

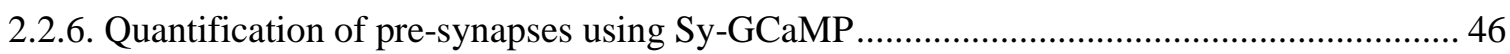

2.2.7. Determining the amount of axons and dendrites using immunocytochemistry................ 48

2.2.8. Immersion microscopy for imaging cAMP levels with AKAR4-cAMPs ...................... 51

2.2.9. Immersion microscopy for imaging cAMP levels with cAMPr .................................. 53

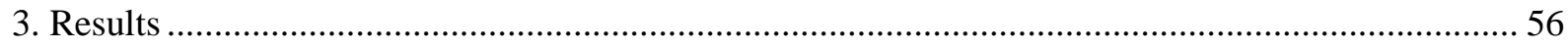

3.1. Optimization of the experimental setup ...................................................................... 56

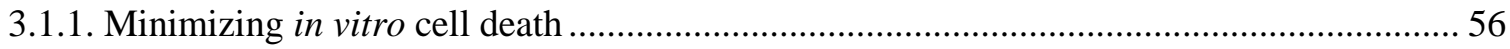

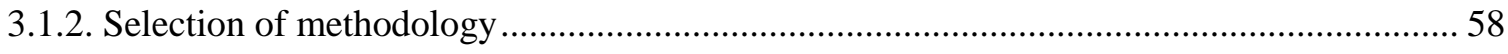

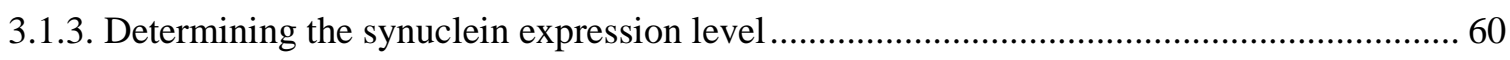

3.2. $\alpha$-synuclein overexpression reduces the frequency of neuron network bursting in culture ....... 61

3.3. The cell culture supernatant of neurons overexpressing $\alpha$-synuclein contains up to $0.36 \mu \mathrm{M}$ released extracellular $\alpha$-synuclein.

3.4. Neuronally produced extracellular $\alpha$-synuclein does not cause the reduction of neuron network bursting. 66

3.5. $\alpha$-synuclein overexpression reduces the number of active pre-synapses and axons 69

3.6. $\alpha$-synuclein overexpression reduces the level of cytosolic cyclic AMP 71 
3.7. The $\beta$-synuclein $\mathrm{P} 123 \mathrm{H}$ mutant reduces the frequency of network bursts, whereas wild-type $\beta$ synuclein and the $\beta$-synuclein V70M mutant do not.

3.8. Tau protein reduces the frequency of network bursts, reduces the level of cAMP and the amount of axons, synapses and dendrites.

4. Discussion 80

4.1. The overexpression of $\alpha$-synuclein reduces the frequency of neuron network rhythmic activity in vitro.

4.1.1 The observed reduction of rhythmic activity frequency by $\alpha$-synuclein occurs sooner than similar observations in vivo

4.2. Extracellular $\alpha$-synuclein was not involved in reducing the frequency of network bursting, contradicting previous results

4.3. The reduction in burst frequency was correlated with diminishments in structures responsible for neuronal connectivity

4.3.1 The observed reduction of axons and synapses in response to $\alpha$-synuclein overexpression is coherent with results of previous studies

4.4. $\alpha$-synuclein overexpression reduces the level of cyclic AMP, a signaling molecule which regulates the growth of axons and the formation of synapses

4.5. Overexpression of the $\mathrm{P} 123 \mathrm{H} \beta$-synuclein mutant reduces the frequency of rhythmic activity by disrupting cellular connectivity, which is similar to the observed impact of $\alpha$-synuclein overexpression.

4.5.1 The non-toxicity of wild-type $\beta$-synuclein and the V70M $\beta$-synuclein mutant is explainable in light of the culture model used in this study

4.6. Synucleinopathy-related disruptions of rhythmic activity in vivo could be explained by a reduction of connectivity, as was observed in this study in vitro

4.7. The disruption of rhythmic activity by tau protein overexpression is accompanied by reductions in connectivity......

4.7.1 The cAMP reduction in response to increased levels of tau overexpression is a novel observation in the context of mammalian neurons

5. Abstract

6. References

7. Supplementary materials .

8. List of abbreviations 


\section{Introduction}

Rhythmic activity is a type of brain activity that involves repeated depolarization of neuronal networks, which is associated with a number of important brain functions, including the regulation of movement and cognition ${ }^{1}$. Notably, rhythmic activity alterations are relevant to disease, as they have been associated with specific brain syndromes, including epileptic seizures and dementia ${ }^{2,3}$. Furthermore, rhythmic activity is also known to be impaired in patients with neurodegenerative disorders, which are nervous system disorders involving a progressive deterioration of nervous system functioning ${ }^{4}$. These include neurodegenerative disorders such as Parkinson's disease (PD), dementia with Lewy bodies (DLB) and Alzheimer's disease (AD), which are affiliated with different symptoms, with common ones including dementia, mood disruptions and motor problems ${ }^{5-7}$.

Notably, rhythmic activity can be measured using quantitative electroencephalography (qEEG) and is divisible into many forms, which differ in terms of their frequency and their relative prevalence to each other. ${ }^{4,8,9}$. Rhythmic activity can thus be divided into higher frequency waves (alpha waves, beta waves) and lower frequency waves (delta waves, theta waves). Importantly, the specific type of rhythmic activity alteration that has been observed in neurodegenerative disorders is qEEG slowing, which involves an increased prevalence of lower frequency waves (associated with sleep and low attentiveness) and a decreased prevalence of higher frequency waves (associated with attentiveness) ${ }^{3,10-12}$.

Interestingly, qEEG studies have further revealed connections between the disruption of rhythmic activity and the presence of aggregating proteins associated with neurodegenerative disorders, such as $\alpha$-synuclein and microtubule-associated protein tau (tau protein). Thus, it has been shown that increased levels of phosphorylated $\alpha$-synuclein are correlated with an increased prevalence of lower frequency rhythmic activity in PD patients ${ }^{13}$. Furthermore, it has been shown that increased levels of cerebrospinal fluid (CSF) tau protein are correlated with rhythmic activity disruptions also in AD patients ${ }^{14,15}$.

The exact explanation for the rhythmic activity disruptions is not known. However, studies of rhythmic activity in vitro as well as studies using computer modelling have shown that its generation is dependent on a number of parameters, including neuronal connectivity ${ }^{16-20}$. It has thus been observed that synchronized rhythmic activity in vitro does not occur at all when the neurite coverage is very low (10\% of the culture plate) ${ }^{18}$. Rhythmic activity in vitro can also be abolished by disrupting neurite functioning, such as when neuronal microtubules are depolarized ${ }^{17}$.

Importantly, several studies exist that have shown that disruptions of connectivity exist in neurodegenerative disorders. For instance in PD, it has been observed that connecting structures (neurites and synapses) are destroyed or exhibit a disrupted physiology ${ }^{21-33}$. Beyond this, subtle disruptions of connectivity-related cellular systems have been observed as well. Studies have thus shown 
that the metabolism of cyclic adenosine monophosphate (cAMP) is disrupted in several PD models, with cAMP being a signaling molecule that plays a role in a variety of pathways, including those regulating neuronal connectivity ${ }^{4,34-38}$.

\subsection{Neurodegenerative disorders}

Neurodegenerative disorders are a group of nervous system disorders, including dementia with Lewy bodies (DLB), Parkinson's disease (PD) and Alzheimer's disease (AD), which are characterized by a gradual degeneration of nerve cells, leading to a disruption of nervous system functioning ${ }^{4-7}$. The impact of these diseases can be severe, as their progression can lead to dementia or motor dysfunction, in turn causing disability ${ }^{5-7}$.

Dementia is a condition characterized by cognitive dysfunction and memory loss, which in most cases is progressive, increasing in severity over time. To illustrate its severity, the annual societal burden caused by dementia is thought to be the greatest of all nervous system disorders, exceeding the burden of diseases such as stroke, traumatic brain injury and epilepsy ${ }^{39}$. As a further illustration, dementia is though to affect tens of millions of people, with an annual worldwide cost of 818 billion dollars ${ }^{40}$. Importantly, the most common cause of dementia are neurodegenerative disorders, with $\mathrm{AD}$ being responsible for $70 \%$ of all cases and DLB for $5 \%$ of all cases ${ }^{41,42}$. PD causes some cases of dementia as well, as it is known to be associated with cognitive symptoms in late-stage disease ${ }^{7}$.

Motor dysfunction caused by neurodegeneration can take a variety of forms and can include problems of movement, gait or body posture. A variety of neurodegenerative disorders can cause motor dysfunction, including Huntington's disease (HD), amylotrophic lateral sclerosis (ALS) and in some cases, DLB and AD ${ }^{7,43-45}$. However, the neurodegenerative disorder that is most associated with motor dysfunction is PD, which is present in $2-3 \%$ of people aged 65 or older ${ }^{6}$. Just like dementia, motor symptoms induced by neurodegenerative disorders represent a major burden on society. This can be observed from the fact that the annual cost of PD in the USA alone has been estimated to be 15 billion dollars ${ }^{46}$.

\subsubsection{Parkinson's disease (PD) and Parkinson's disease dementia (PD-D)}

PD is a disorder that primarily impacts parts of the brain associated with motor functioning, such as the substantia nigra $(\mathrm{SN})$, but can also target other structures in late-stage $\mathrm{PD}$, such as the cerebral cortex. PD is associated with a variety of symptoms, including motor symptoms, olfactory symptoms, gastrointestinal symptoms and cognitive symptoms ${ }^{6,47}$.

Of the four types of symptoms, motor symptoms are considered to be primary and emerge earlier in disease progression. The key symptom of PD is bradykinesia, where the patient experiences slow voluntary movement initiation. Alongside bradykinesia, a second motor symptom - either muscular rigidity, rest tremor or postural instability - is required to be present for an official diagnosis ${ }^{6,47}$. 
Differently from motor symptoms, olfactory symptoms and gastrointestinal symptoms are not primary symptoms, but are instead prodormal symptoms of PD. The olfactory symptom characteristic of prodormal PD is anosmia (absence of smell). Meanwhile, several gastrointestinal symptoms are also characteristic of prodormal PD, including constipation, with PD patients exhibiting a spread of Lewy bodies throughout the enteric nervous system ${ }^{6,47,48}$.

Finally, cognitive symptoms are characteristic of late-stage PD and include dementia, memory deficits, mood disorders, pain and disruption of autonomic functions. As in the case of the other symptoms, the arrival of these symptoms is associated with the spread of Lewy bodies, in this case to the cerebral cortex. Notably, two key subtypes of PD are associated in particular with cognitive symptoms. These two subtypes are Parkinson's disease with mild cognitive impairment (PD-MCI) and Parkinson's disease dementia (PD-D), with PD-MCI being a milder precursor of PD-D ${ }^{6,47,48}$.

No cure for PD is known to exist. However, alleviation for PD can be achieved by the use of drugs targeting dopaminergic signaling, such as levodopa, catechol-o-methyl-transferase inhibitors, monoamine oxidase inhibitors and dopamine agonists. Furthermore, deep brain stimulation is an effective surgical treatment and can be used as a substitute treatment for pharmacological approaches ${ }^{6}$. For non-motor symptoms, drugs similar to those used for DLB can be effective, including drugs such as acetylcholine inhibitors, N-methyl D-aspartate (NMDA) receptor antagonists, selective serotonin reuptake inhibitors and anti-psychotics ${ }^{49}$.

\subsubsection{Dementia with Lewy bodies (DLB)}

DLB is a neurodegenerative disorder that impacts a variety of brain areas and is distinguished by a variety of symptoms. The first of these is fluctuating cognition, where patients experience delirium, which is a syndrome involving loss of attention and alertness. The second symptom is visual hallucinations, which is present in as many as $80 \%$ of the patients. The third symptom is rapid eye movement (REM) sleep behavior disorder, which often occurs earlier than the other symptoms and involves the loss of the paralysis characteristic of REM sleep. Finally, the fourth symptom of DLB is parkinsonism, meaning the presence of one or more symptoms shared with PD ${ }^{50}$.

PD and DLB thus share several symptoms - including both cognitive disruptions and parkinsonian symptoms. Nevertheless, the two disorders are distinct, as motor symptoms are primary in PD, whereas cognitive symptoms are primary in DLB. The diagnostic criteria used for differentiation between these disorders is the time of cognitive symptom onset in relation to the time of parkinsonian symptom onset. More specifically, if cognitive symptoms emerge 1 year after the onset of motor symptoms or later, the patient is diagnosed as having PD-D. Otherwise, he is diagnosed as having DLB ${ }^{49}$.

No cure for DLB is known to exist, although the alleviation of symptoms is possible. Thus, cognitive problems can be alleviated using acetylcholinesterase inhibitors, which are drugs also used for symptom 
alleviation in $\mathrm{AD}$ patients. Other symptoms, such as hallucinations and depression, can be alleviated with antipsychotics and antidepressants, respectively ${ }^{7}$.

\subsubsection{Alzheimer's disease (AD) and mild cognitive impairment (MCI)}

Alzheimer's disease is a neurodegenerative disorder that disrupts the cortex, which is characterized by three categories of dysfunctions. The first of these are cognitive disruptions, which take the form of memory loss and severe dementia. The second of these are behavioral disruptions, such as mood fluctuations, hallucinations and delusional thinking. The third of these are daily living disruptions, where patients have problems with basic everyday activities ${ }^{5,51}$.

AD cannot be cured, but can be alleviated with a variety of treatment strategies ${ }^{5}$. Of these, one approach for therapy aims to reduce the formation of amyloid beta $(A \beta)$ plaques using antibodies and has proven to be successful in human trials ${ }^{52}$. Other approaches that have been considered include inhibitors of 5-hydroxytryptamine receptors and deep brain stimulation, the same treatments as used in PD. Patient symptoms can also be alleviated by means of physician-supervised lifestyle changes, involving alteration in daily activities, diet and exercise ${ }^{53}$.

Mild cognitive impairment (MCI) is a cognitive disorder characterized by greater cognitive decline than similarly aged patients but without interference in daily life activities. MCI is closely linked to AD, with some studies even considering it to be a prodormal form of $\mathrm{AD}$. This is primarily caused by the fact that $\mathrm{MCI}$ patients have an increased risk of disease progression to $\mathrm{AD}$, with an 11-33\% risk of developing dementia over 2 years ${ }^{54,55}$

\subsection{Neurodegenerative disorders and aggregating proteins}

Notably, PD, DLB and AD are all associated with characteristic types of aggregates in patient tissue, which are formed due to aberrant protein aggregation. The aggregates considered distinctive for PD and LBD are $\alpha$-synuclein containing inclusions known as Lewy bodies ${ }^{6}$. Meanwhile, the aggregates considered distinctive for $\mathrm{AD}$ are tau protein containing neurofibrillary tangles and $\mathrm{A} \beta$-containing amyloid plaques ${ }^{5}$. Importantly however, there is an overlap between disorders - stages or subtypes of the diseases might be associated with different types of aggregates, not just the one particularly associated with that disease ${ }^{56-58}$.

\subsection{1. $\alpha$-synuclein}

$\alpha$-synuclein is a protein composed of 140 amino acids that is known to occur in synapses, the cytosol and the nucleus. Its exact role has not been demonstrated, but it is known to be overexpressed broadly in the central nervous system and is thought to be involved in modulating synaptic vesicle clustering and release, mitochondrial function and intracellular trafficking 6,33,59,60. It is also involved in signaling, as $\alpha$-synuclein has been shown to inhibit phospholipase $\mathrm{D}$, the enzyme responsible for producing the phosphatidic acid signaling molecule. Despite its variety of roles, studies of rodents have suggested that 
the presence of $\alpha$-synuclein is not required for organism survival, as $\alpha$-synuclein knockout mice have been shown to be viable, although some studies have suggested that the mice experience disruptions in synaptic functioning and cognition ${ }^{32,61}$.

In terms of structure, $\alpha$-synuclein has three domains. The first is the $\mathrm{N}$-terminal domain, which is known to contain hexamer amino acid repeats (KTKEGV) and is thought to be involved in lipid binding. The second is the non amyloid-component (NAC) domain, which is thought to be involved in aggregate formation, with characteristic synuclein aggregates including the smaller oligomers, the intermediary protofibrils and the large insoluble amyloid fibrils that make up Lewy bodies ${ }^{62-64}$. The final domain of $\alpha$-synuclein is the C-terminal domain, which is rich in acidic glutamate and aspartate residues, which are thought to be involved in interactions with metal ions and other proteins.

Importantly, the pathogenic role of $\alpha$-synuclein is indicated by an increased risk of developing PD in cases where patients contain multiplications of the $\alpha$-synuclein gene in their genome ${ }^{65}$. Mutations in the SNCA gene can also be disease-associated, such as the A53T and A30P mutations, which are thought to increase the rate of aggregate formation ${ }^{66}$.

\subsubsection{Lewy bodies}

Lewy bodies are intracellular aggregates that are composed of aggregated $\alpha$-synuclein fibrils alongside other proteins ${ }^{67}$. These proteins include autophagy or protein degradation proteins, synapse proteins, cytoskeletal proteins and mitochondrial proteins, with specific examples including ubiquitin, synaptophysin and cytochrome $\mathrm{C}^{68,69}$. Lewy neurites are aggregates related to Lewy bodies, which have a more elongated shape and are mainly localized in neurites. Lewy bodies are distinctive in the cells of both PD and LBD patients, but the distribution of these aggregates in the nervous system and the time of their emergence during disease progression is different between the two diseases ${ }^{70,71}$.

In terms of structure, Lewy bodies are composed of filaments, which are oriented radially, aligned from the center towards the edge of the structure ${ }^{72}$. Lewy bodies have been suggested to have three distinct layers, differentiated from each other by protein composition. The innermost layer is mainly composed of ubiquitin, which is surrounded by a layer of $\alpha$-synuclein and finally by the outermost layer, which is formed by neurofilaments ${ }^{73}$.

In the case of PD, Lewy bodies spread from brainstem structures to the midbrain and finally to cortical structures. More specifically, the aggregates spread from the dorsal IX/X nucleus to the locus coeruleus, then to the substantia nigra, subsequently to the mesocortex and finally to the neocortex ${ }^{70}$. In the case of DLB, early development of Lewy body pathology in the cortex is considered characteristic of the disease ${ }^{71}$. 


\subsection{3. $\beta$-synuclein and $\gamma$-synuclein}

$\beta$-synuclein is a protein of the synuclein family that is overexpressed in synapses and is present in many regions of the central nervous system. Its function is not fully known, but $\alpha$-synuclein and $\beta$-synuclein knockout mice have been shown to have mild alterations of dopamine levels, suggesting that $\beta$-synuclein is involved in the regulation of synapse function, which is similar to the role of $\alpha$-synuclein ${ }^{74}$. It is also involved in cell signaling, as it is known to be an inhibitor of phospholipase $\mathrm{D}^{75}$. In terms of structure, $\beta$-synuclein is similar in size and domain composition to $\alpha$-synuclein, however the $\beta$-synuclein protein segment which is homologous to the $\alpha$-synuclein NAC domain is altered in terms of its amino acid composition ${ }^{76}$.

Notably, $\beta$-synuclein is an aggregating protein, although it has a lower aggregation propensity than $\alpha$-synuclein ${ }^{77}$. Its role in synucleinopathies is unclear. On the one hand, $\beta$-synuclein has been shown to be neurotoxic in certain contexts, for instance when it is overexpressed in nigral dopaminergic neurons ${ }^{78}$. On the other hand, $\beta$-synuclein is absent from Lewy bodies, the distinctive type of pathological protein aggregate in $\mathrm{PD}{ }^{6,79}$. Some studies have also claimed that $\beta$-synuclein could be a neuroprotective agent and suggest that it could inhibit $\alpha$-synuclein aggregation ${ }^{80-82}$.

Unlike $\alpha$-synuclein, disease-related $\beta$-synuclein multiplications have not been discovered. However, two $\beta$-synuclein mutants have been found to be disease-associated, increasing the risk of developing DLB the V70M and P123H $\beta$-synuclein mutants ${ }^{83}$. These have been shown to have a variety of functions, including inducing cell death, causing axonal loss and precipitating alterations in mitochondrial shape and motility ${ }^{84}$.

$\gamma$-synuclein is the third member of the synuclein family and is a protein localized in the cytoplasm, the perinuclear area and the nucleus ${ }^{85}$. Its function is not fully known, but $\gamma$-synuclein knockout mice exhibit a reduction of dopaminergic neurons when compared to wild-type animals ${ }^{86} \cdot \gamma$-synuclein is also thought to have non-neuronal functions, including promotion of cancer cell survival and lipolysis ${ }^{87,88}$. In terms of structure, $\gamma$-synuclein exhibits some homology with the other synucleins, especially in the $\mathrm{N}$-terminal domain. However is more divergent from the other two synucleins than $\alpha$-synuclein is from $\beta$ synuclein ${ }^{76,89}$.

In terms of aggregation, $\gamma$-synuclein can aggregate, but does so at a reduced rate when compared to $\alpha$-synuclein ${ }^{77}$. It is also not a component of Lewy bodies ${ }^{79}$. Compared to $\alpha$-synuclein and $\beta$-synuclein, the connection between $\gamma$-synuclein genetic variants and neurodegenerative disorders is less known, although, one study has indicated that a point mutation in a non-coding region adjacent to the $\gamma$-synuclein gene $(S N C G)$ locus increases diffuse Lewy body disease risk ${ }^{90}$. 


\subsubsection{Microtubule-associated protein tau (tau protein) and neurofibrillary tangles}

Tau protein is a protein which regulates the stability of axonal microtubules and is encoded by the microtubule-associate protein tau (MAPT) gene ${ }^{91}$. MAPT knockout mice are viable, but have been shown to have a reduced axonal growth rate, alongside cognition and motor deficits ${ }^{92,93}$. Tau protein is distinguished by its ability to aggregate, which is known to be enhanced by tau protein phosphorylation and the presence of extracellular matrix proteins, such as glycosaminoglycans ${ }^{94}$.

Several types of protein modifications are associated with the tau protein of neurofibrillary tangles. The most distinctive is hyperphosphorylation, which is strongly related to tangle generation ${ }^{95}$. Glycosylation is also though to be causative of tau protein aggregation, as it makes the protein more prone to phosphorylation ${ }^{96}$. Other type of protein modifications, such as ubiquitination, glycation, polyamination and nitration have also been described for tau protein ${ }^{97}$.

Neurofibrillary tangles are fibrillar aggregates composed of tau protein that are found inside cell bodies and neurites. In terms of structure, the tangle is primarily composed of paired helical filaments and straight filaments ${ }^{98,99}$. Alongside tau protein, other proteins are present as well, such as ubiquitin and glial fibrillary acidic protein ${ }^{97}$.

\subsubsection{Amyloid beta (Aß) and amyloid plaques}

Amyloid precursor protein (APP) is an integral membrane protein composed of around 700 amino acids that is thought to play a role in synapse formation and synapse activity ${ }^{100}$. The cleavage product of APP, $\mathrm{A} \beta$, is thought to play a role as well, with released soluble $A \beta$ peptides acting as a form of signaling to nearby cells ${ }^{101,102}$. Knockouts of APP have revealed that a proportion of the animals survive into adulthood in the absence of APP, although they suffer neurological problems, such as long-term memory deficits ${ }^{103}$.

Amyloid plaques are extracellular aggregates composed primarily of aggregated amyloid beta (A $\beta$ ) peptides but also other proteins, such as extracellular matrix proteins and ubiquitin ${ }^{5,104}$. As in the case for synuclein, $A \beta$ peptides assume a fibrillar amyloid structure in this aggregate ${ }^{105}$. In terms of their evolution, amyloid plaques form around amyloid deposits, with inflammation-related components becoming associated with the aggregate after it grows in size ${ }^{106}$.

Amyloid plaque formation is also associated with $\gamma$ secretase, a protein encoded by the AD-associated presenilin-1 and presenilin- 2 genes. This protein plays a role in A $\beta$ peptide generation, as it cleaves the substrate protein of $A \beta$ - amyloid precursor protein (APP). Importantly, mutated $\gamma$-secretase can alter the amino acid composition of $A \beta$ peptides by alternative cleaving of the protein, which in some cases leads to the generation of more aggregation-prone hydrophobic peptides ${ }^{5}$. 


\subsection{Rhythmic and intrinsic activity}

\subsubsection{In vivo rhythmic activity}

Rhythmic activity is a type of activity characterized by repetitive depolarization of neuronal networks. In the brain, rhythmic activity is highly diverse and can be measured with the qEEG method, which measures electric potential fluctuations at the scalp, which are in turn generated by membrane potential changes taking place at the synapses of pyramidal neurons in the cerebral cortex ${ }^{4}$.

Using qEEG, several different types of rhythmic activity have been identified, which are differentiated from each other based on their frequency (Figure 1). From highest to lowest frequency, these are - beta waves, alpha waves, theta waves and delta waves. Beyond these, there also exist types of rhythmic activity that occur primarily in juvenile animals, such as the low frequency $(0.1 \mathrm{~Hz})$ hippocampal giant depolarizing potentials (GDP) and cortical early network oscillations (cENOs) ${ }^{107-109}$. Importantly, each type of rhythmic activity can be associated with different types of brain function - higher frequency rhythmic activity is generally associated with attentiveness, whereas lower frequency activity is associated with various sleep phases and inattentiveness ${ }^{8}$.

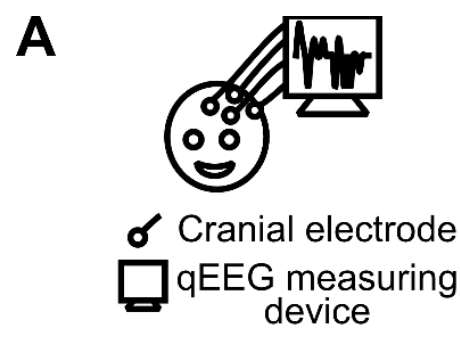

C

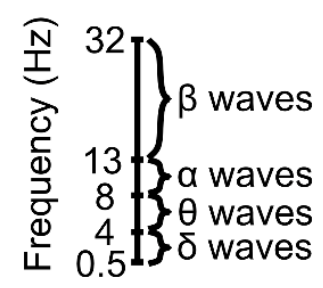

B

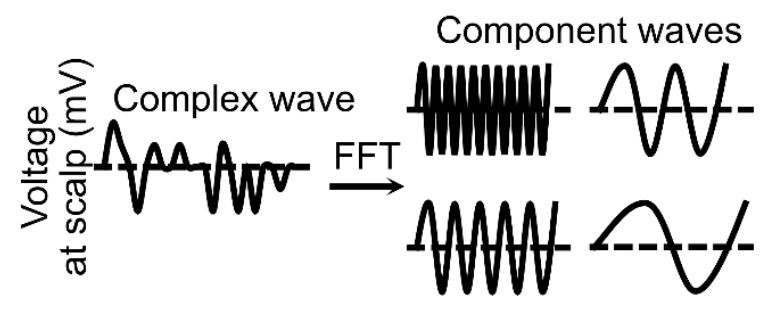

D

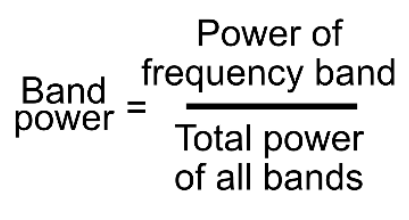

E General pattern for dementia cases

\begin{tabular}{|c|c|}
\hline Wave & Bandpower \\
\hline$\beta$ & $\downarrow$ \\
\hline$\alpha$ & $\downarrow$ \\
\hline$\theta$ & $\uparrow$ \\
\hline$\delta$ & $\uparrow$ \\
\hline
\end{tabular}

Figure 1. Basic concepts of quantitative electroencephalography (qEEG).

(A) Illustration of qEEG measurement using s cranial electrode

(B) Depiction of qEEG signal decoding. An illustration of a complex wave pattern (left panel), which is converted by a fast Fourier transform method (FFT) into component waves (right panel). Y-axis of the left panel = fluctuations of the membrane potential at the scalp

(C) Frequency ranges of different brain waves. Vertical scale $=$ rhythmic activity frequency $(\mathrm{Hz}) . \beta$ waves $=$ beta waves, $\alpha$ waves $=$ alpha waves, $\theta$ waves $=$ theta waves, $\delta$ waves $=$ delta waves.

(D) Formula used for determining the band power of qEEG signals.

(E) Table illustrating the basic pattern of bandpower changes in cases of dementia. Green arrow = increase, red arrow $=$ decrease.

Importantly, these brain activities are generated in different areas of the brain. For instance, alpha and theta waves originate in the thalamus and are generated by thalamocortical neurons. Meanwhile, delta waves and slow waves can originate in either the thalamus or the cortex ${ }^{110}$. A variety of mechanisms 
are used for the generation of these waves, such as gap junctions, hyperpolarization-activated cyclic nucleotide gated (HCN) channels and voltage-gated calcium channels ${ }^{110}$.

As one example of how rhythmic activity is generated in vivo, slow waves (cortical low frequency waves similar to delta waves) are generated by a mechanism involving alternating neuronal excitability. More specifically, these waves are thought to be induced by neurons known as layer 5 regular spiking pyramidal neurons, which are large neurons that undergo bursting. During bursting activity, these cells depolarize first and then depolarize other neurons in the cortex ${ }^{110,111}$. However, after this excitation, a high proportion of the target neurons afterhyperpolarize and becomes less excitable, thus inducing a break in the activity. Then, the afterhyperpolarization dissipates, excitatory inputs again increase in magnitude, triggering another round of depolarization, which in turn triggers another round of afterhyperpolarization, thus creating a rhythm ${ }^{110,112}$.

\subsubsection{In vitro rhythmic activity}

Neurons exhibit rhythmic activity not only in in vivo systems, but also in culture preparations, such as those prepared from embryonic rat brains, with the standard practice being to generate such cultures from rat embryos on E18 or E16 $6^{86,113,114}$. For the seeding of these cultures, disassociated cortical neurons are transferred to culture plates, following which they develop in culture from round cells with small projections into cells exhibiting many of the attributes of mature neurons, including dendrites, axons, synapses and neuronal polarity ${ }^{114}$. In vitro rhythmic activity has been named in various different ways, however one term that is frequently used to describe it is bursting or network bursting ${ }^{86,115}$.

A variety of attributes are important for determining the development of these cultures. These include both the density of the culture and its connectivity in terms of the amount of neuritic structures ${ }^{18,114,116}$. Importantly, neuron cultures are unable to develop rhythmic activity in cultures which are seeded in overly low density or with an overly sparse neuritic network. For instance, one study has claimed that no rhythmic activity is generated in cultures with less than 50 neurons per $\mathrm{mm}^{2}$ as well as cultures where neurites cover $10 \%$ or less of the plate surface ${ }^{18}$.

In vitro, the mechanism behind rhythmic activity is thought to be the spontaneous depolarization of single cells, which triggers a depolarization that is then spread to the rest of the culture ${ }^{16,117}$. A variety of factors are thought to be involved in regulating this spread, with the activity being dependent on glutamate receptors, such as $\alpha$-amino-3-hydroxy-5-methyl-4-isoxazolepropionic acid (AMPA) and NMDA receptors ${ }^{86,118,119}$. Notably, gamma-aminobutyric acid (GABA) receptors are also thought to play a role in the generation of network bursts, with these channels contributing an excitatory drive in early culture, which helps to generate network bursting ${ }^{119}$. However, in later stages of the culture, GABA receptors are not required for the maintenance of network bursting and instead shift to have an inhibitory impact ${ }^{86,118,119}$. 
A variety of membrane channels are also thought to regulate the rhythmic activity of neuron cultures. These include voltage-gated sodium and potassium channels, which are responsible for generating the depolarization and hyperpolarization that is characteristic of action potentials ${ }^{4}$. A further group are the voltage-gated calcium channels, such as L-type and T-type calcium channels, which regulate the neuron's transition from resting membrane potential to its threshold potential, which is the potential at which action potentials begin to be generated ${ }^{4,120}$. A final group of relevant channels are the cAMPregulated $\mathrm{HCN}$ channels, which regulate membrane potential at more negative values ${ }^{38,121,122}$.

Importantly, rhythmic activity is not generated in neuronal culture upon plating and instead develops over time. This is indicated by a study by Opitz et al., which showed that the level of synchronized activity in early culture is low, with about half of all active neurons being synchronously active at DIV9 118. The level of synchronized activity then increases over time, as at DIV15, all active neurons become synchronized ${ }^{118}$. Interestingly, the level of connecting structures in neuron cultures increases during the same timeframe, which possibly explains the development of synchronized network activity, due to connectivity being a key parameter of network bursting ${ }^{16-19,114,123}$.

Relevantly for this study, in vitro rhythmic activity is easy to measure accurately using several different methods. In vitro, two approaches are commonly used. In one approach, cells are prepared on plates equipped with a microelectrode array, which permit the direct measurement of the membrane potential of the neurons ${ }^{16}$. This method however also has a disadvantage, in that it is difficult to determine how many neurons produce the signals detected at the microelectrodes and where these neurons are located ${ }^{124}$.

In the second approach, calcium sensors are used instead, such as dyes or genetically encoded calcium sensors such as GCaMP ${ }^{113,118,125}$. Importantly, calcium sensors can be used because the depolarizations characteristic of rhythmic activity are followed by a calcium influx. Notably, this method has the advantage of producing one signal per neuron, which is simple to analyze and register using fluorescence microscopy. The disadvantage of this method however is that using this method, information about the membrane potential of the neuron is not recorded.

\subsubsection{The role of $\alpha$-synuclein and tau protein in regulating rhythmic activity}

As mentioned, rhythmic activity disruptions exist in neurodegenerative disorders, including PD, DLB and $\mathrm{AD}$, the disorders associated with either $\alpha$-synuclein or tau protein. These disruptions are detected using qEEG and take the form of an increased prevalence of low frequency activity (also referred to as qEEG slowing) ${ }^{3,10-12}$. In the case of PD, which is primarily a motor disorder, qEEG slowing is associated with patients that exhibit cognitive symptoms ${ }^{10,126,127}$. Interestingly, qEEG slowing has also been claimed to be predictive of disease symptoms, such as the onset of dementia ${ }^{128-130}$.

The relationship between $\alpha$-synuclein and disruptions of rhythmic activity has been most comprehensively studied in a publication by Caviness et al., which observed that increased levels of 
phosphorylated $\alpha$-synuclein were significantly correlated with an increased prevalence of lower frequency qEEG waves (delta waves, theta waves) and with a decreased prevalence of higher frequency qEEG waves (alpha waves, beta waves) ${ }^{13}$.

The impact of $\alpha$-synuclein on rhythmic activity has also been studied in test animals, such as the Thy1$\alpha$-synuclein mouse model. This model involves the use of genetically modified animals, which have been altered to express human $\alpha$-synuclein under the control of the Thy- 1 promoter, increasing the expression of $\alpha$-synuclein 1,5-5 fold ${ }^{131,132}$. Notably, McDowell et al. has observed an increase in the prevalence of lower frequency rhythmic activity using qEEG in these mice, which was similar to the disruption observed in human patients ${ }^{133}$. This study was confirmed by a subsequent study by Morris et al. which furthermore observed reduced network excitability inside Thy1- $\alpha$-synuclein mouse brains ${ }^{134}$.

In addition to in vivo studies, models have also been created to study the impact of $\alpha$-synuclein in vitro, which have focused on the potential impact of recombinant $\alpha$-synuclein artificially aggregated into fibrils. Notably, studies observed that treating the neurons with $0.13-0.50 \mu \mathrm{M}$ recombinant $\alpha$-synuclein aggregates reduces the frequency of network bursting ${ }^{135,136}$.

With regard to tau protein, a study by Smailovic et al. has observed a relationship between increased levels of tau protein and rhythmic activity disruptions in patients. In this study, a wide selection of dementia patients were measured, including patients suffering from $\mathrm{AD}$ and from MCI. More specifically, the rhythmic activity of the patients was measured using qEEG and a sample of their CSF was taken using lumbar puncture. It was thus observed that increased levels of tau were correlated with a decrease in the global field power (the signal strength) of high frequency rhythmic activity (alpha waves and beta waves). Interestingly, such a correlation was not observed in the case of increased levels of amyloid beta, another protein associated with $\mathrm{AD}^{15}$.

The impact of tau protein on rhythmic activity has also been studied in vitro. Thus, a study by Verstraelen et al. has observed that artificial overexpression of tau protein in cultured hippocampal neurons reduces the frequency of rhythmic activity in this model system. Interestingly, this study also investigated possible explanations behind the frequency reduction, which however yielded contradictory results - tau protein overexpression was observed to reduce the amount of neurites but not synapses ${ }^{17}$.

\subsubsection{Intrinsic activity and functional magnetic resonance imaging (fMRI)}

Intrinsic activity is a type of brain activity that is not triggered by an external stimulus, which can be studied using a method known as functional magnetic resonance imaging (fMRI) ${ }^{137}$. One of the most common types of fMRI is blood oxygen level dependent (BOLD) fMRI, which is a method that indirectly detects brain activity by analyzing blood flow in the head. More specifically, it measures the presence of oxygen-deprived blood, which is taken as an indicator of metabolic activity in the surrounding brain tissue. The BOLD fMRI signal is then used to detect functional connectivity (FC) 
between different brain regions, with two parts of the brain being connected if they are active simultaneously. Notably, functional connectivity can be utilized further, for instance to identify the presence of different brain networks, such as the default mode network ${ }^{4,138,139}$.

On a technical level, MRI involves exciting hydrogen ions with a magnetic field, which will cause the particles to emit radio signals that can then be recorded. Importantly, the magnetic properties of the surrounding tissue alters this phenomenon - relevantly to BOLD fMRI, oxygenated and deoxygenated blood have different magnetic properties and thus alter the fMRI signal in different ways. This difference in magnetic properties is used in BOLD fMRI to identify metabolically active brain tissue, as active brain tissue consumes oxygen and produces local increases of deoxygenated blood, which then alter the fMRI signal. After identification of metabolically active brain tissue, the activity of different areas is then temporally correlated to identify those areas that are functionally connected ${ }^{4,139}$.

Importantly, fMRI has been used to study neurodegenerative disorders as well, with the goal being to detect alterations in the functional connectivity between brain regions. In the case of PD, connectivity has been shown to be altered in various circumstances, with the default mode network (DMN) being one of the networks disrupted in PD patients ${ }^{140-142}$. In the case of DLB, disruptions of the fronto-parietal network and of the visual cortex have been observed, whereas the DMN is not impacted ${ }^{143-145}$. In the case of $\mathrm{AD}$, several studies show disruptions of the DMN and of the connectivity between the hippocampus and other brain regions ${ }^{146-150}$.

\subsection{Neurites and synapses in PD, DLB and AD}

\subsubsection{The impact of $\alpha$-synuclein on neurites}

Neurite loss has been identified as a symptom in several synucleinopathies, including PD and DLB, with one example of afflicted neurites being nigrostriatal fibers, which connect the substantia nigra and the striatum ${ }^{5-7,47,151}$. Importantly, neurite death in PD is thought to be dying-back process, which involves the death of synapses and neurites before the death of the neuronal somata ${ }^{152-154}$. Aside from PD, neurites are also impaired in DLB, with neurite damage being observed in the cerebral cortex ${ }^{27,155}$.

Beyond this, studies exist which have connected neuritic lesions to $\alpha$-synuclein in particular. For instance, increased levels of $\alpha$-synuclein have been shown to induce degradation of both cortical and dopaminergic axons in both cortical and dopaminergic neurons ${ }^{28,156,157}$. Beyond this, more subtle $\alpha$-synuclein related lesions have been described as well, such as the disruption of axonal transport, possibly through interactions with cytoskeletal proteins, including tau protein ${ }^{158-160}$. Interestingly, $\alpha$-synuclein has also been suggested to impair myelination, possibly through an interaction with oligodendrocytes ${ }^{161,162}$.

Notably, studies exist which have indicated that increased levels of $\alpha$-synuclein can impact dendrites. For instance, it has been shown that in the presence of increased levels of $\alpha$-synuclein aggregates, the 
level of oxidant stress is elevated in dendritic structures ${ }^{163}$. Beyond this, $\alpha$-synuclein aggregates have also been shown to cause the outright loss of dendritic spines, although this has been contradicted by a different study, which suggests that $\alpha$-synuclein overexpression increases dendritic spine density ${ }^{164,165}$.

\subsubsection{The impact of $\alpha$-synuclein on synapses}

Importantly, in both diseased and non-diseased animals, $\alpha$-synuclein is a predominantly synaptic protein, which is localized in presynaptic nerve terminals ${ }^{60,166,167}$. The mechanisms for the synaptic localization of synuclein are not fully known, but it is known that it is enhanced by the interaction between synucleins and synaptic lipid rafts - synuclein levels are reduced when lipid rafts are broken down artificially ${ }^{168,169}$. In the synapse, synuclein exists in a variety of forms. It has thus been claimed that $\alpha$-synuclein is monomeric when in the cytosol but adopts a multimeric form when interacting with plasma membrane components, with some studies claiming that multimerization is more specifically promoted by interaction between $\alpha$-synuclein and polyunsaturated fatty acid groups ${ }^{170-173}$.

The function of $\alpha$-synuclein in the synapse is mediated by its ability to bind and interact with a number of synaptic proteins. For instance, $\alpha$-synuclein has been shown to interact with proteins involved in vesicle fusion, including vSNARE and the vesicle-associated membrane protein 2 (VAMP2) ${ }^{174-176}$. The role of synuclein in this mechanism is thought to be involvement in the assembly of the SNARE complex, with rates of SNARE complex assembly reduced in synuclein triple knockout animals ${ }^{175}$.

$\alpha$-synuclein has also been shown to influence synaptic vesicle dynamics, for instance by modulating the size of the synaptic vesicle reserve pool, which is a storage population of vesicles that is mobilized by the synapse for release in cases of strong stimulation ${ }^{4,177,178}$. While $\alpha$-synuclein is not mandatory for the presence or maintenance of the reserve pool, one study done in synuclein knockout mice has suggested that the size of the reserve pool is reduced in these animals under certain scenarios, such as in cases of extensive neuronal stimulation, with mobilization of synapses from the reserve pool also being disrupted ${ }^{32}$. The role of $\alpha$-synuclein in reserve pool maintenance is further supported by studies indicating that $\alpha$-synuclein inhibition using antisense oligonucleotides decreases reserve pool size ${ }^{179}$.

The specific way that synuclein influences the functioning on the reserve pool is not known, but it has been suggested that it involves the interaction of $\alpha$-Synuclein with the cytoskeletal protein actin ${ }^{180}$. Studies have shown that synuclein serves to fine-tune the polymerization of actin, promoting polymerization during synaptic activity and inhibiting polymerization during synaptic inactivity ${ }^{177,181}$. This could in turn impact the mobilization of the reserve pool, as a functioning actin scaffold is required for mobilization of vesicles and for vesicle fusion ${ }^{180,182}$.

Beyond its disruption of the reserve pool of synaptic vesicles, $\alpha$-synuclein has also been shown to interfere with the recycling pool of vesicles, which is a population of vesicles that is mobilized upon moderate neuronal stimulation ${ }^{178}$. More specifically, increased levels of synuclein reduce the mobility 
of this vesicle population and also reduce the mobility of vesicles between synapses, possibly due to an interaction between $\alpha$-synuclein and the protein VAMP2 ${ }^{183,184}$.

$\alpha$-synuclein has also been shown to alter rates of neurotransmitter release, with some studies connecting this observation with an impact of synuclein on vesicle kinetics ${ }^{167}$. More specifically, synuclein knockouts have been shown to have increased levels of dopamine release, whereas animals overexpressing synuclein have reduced levels of dopamine release ${ }^{185-190}$. Studies also exist that suggest that elevated levels of synuclein can impair the release of other neurotransmitters, such as glutamate ${ }^{191}$.

There are indications that $\alpha$-synuclein induced synaptic impairment has a key role in the etiology of PD and DLB ${ }^{167}$. As one example of this, monomeric, multimeric and aggregated synuclein are abundant in the synapses compared to other cellular compartments, with the one synuclein aggregate not common in synapses being Lewy bodies ${ }^{60,192}$. Furthermore, studies have shown that the presence of synucleincontaining aggregates in synapses precedes the presence of somatic Lewy inclusions in dopaminergic neurons ${ }^{193,194}$. Importantly, PD pathology on a cellular level has also been shown to begin at synapses, and then spread to somata ${ }^{152-154}$. Beyond this, $\alpha$-synuclein overexpression induced neurotransmitter release reduction has been argued to be an explanation for the impairments in dopaminergic neurotransmission that are characteristic of $\mathrm{PD}^{6,167}$.

\subsubsection{The impact of tau protein on neurites and synapses}

In $\mathrm{AD}$, neurites are lost in a variety of areas, including the white matter of the cortex and the corpus callosum, with neurite loss being thought to involve a dying-back mechanism similar to the one observed for $\alpha$-synuclein $25,31,195$. Importantly, both types of AD aggregate are associated with neurites, with neurofibrillary tangles containing tau protein, which is associated with axonal microtubules, whereas amyloid plaques are often interlaced with dystrophic neurites ${ }^{196}$. With regard to synapses, loss of synapses in brain regions impaired by $\mathrm{AD}$ is thought to be a correlate of cognitive impairment, with synaptic dysfunction thought to be triggered by a number of mechanisms, such as oxidative stress ${ }^{197}$.

In its non-pathological form, tau protein is known to serve a number of axonal functions. These include altering the polymerization of microtubules, with tau protein thought to stabilize microtubule assemblies and thus playing a role in the genesis of neurites ${ }^{198}$. By contrast, tau protein overexpression is thought to disrupt axonal transport (although this is disputed in some studies) and lead to axon degeneration, possibly through the formation of the more toxic phosphorylated tau protein ${ }^{5,17,29,199-201}$. Interestingly, tau protein overexpression has also been shown to induce gliosis ${ }^{200}$.

Non-pathological functions for tau protein in the synapse are less known than functions for tau protein in the neurites, although tau protein is known to be contained in the synapse, with one study suggesting that neurons lacking tau exhibit a reduction in synaptic bouton size ${ }^{202}$. Beyond this, tau protein has also been suggested to have other roles, such as regulation of synaptic receptor activity ${ }^{203}$. As in the case of neurites, tau protein can also have a pathological role in the synapse. Thus for instance, the presence of 
hyperphosphorylated or oligomeric tau protein is thought to be associated with a loss of synapses ${ }^{204}$. In addition to this, tau protein is also though to interfere with synaptic functions in more subtle ways as well, such as by disrupting synaptic vesicle kinetics and neurotransmitter release rate ${ }^{205}$.

\section{5. cAMP and HCN channels}

\subsubsection{Cyclic adenosine monophosphate (cAMP)}

cAMP is a cellular secondary messenger that is involved in signaling ${ }^{206}$. In neurons, it can activate a variety of other messaging proteins, such as kinases of the protein kinase A (PKA) family, mitogenactivated protein (MAP) kinases and HCN membrane channels ${ }^{38,207}$. Notably, cAMP is also a signaling target, for instance of neurotransmitter receptor signaling pathways ${ }^{208}$. More specifically, these pathways are known to influence cAMP levels by altering the activity of enzymes involved in cAMP production and breakdown - adenyl cyclases (AC), which synthesize cAMP from adenosine triphosphate (ATP), and phosphodiesterases (PDE), which break cAMP down into adenosine monophosphate (AMP) ${ }^{207}$.

Through its role in signaling, cAMP is involved in regulating a variety of neuronal functions. As one example, cAMP is involved in the regulation of synaptic long term potentiation (LTP), which is the process where synapses are strengthened in response to recent activity. In this process, NMDA receptors in synapses are repeatedly activated, leading to a calcium influx that activates a calcium-dependent adenyl cyclase. This subsequently increases the level of cAMP, leading to the activation of PKA and cAMP response element binding protein (CREB). The activation of CREB then alters gene transcription, leading to alterations in synaptic growth and function (Figure 2) ${ }^{4}$.

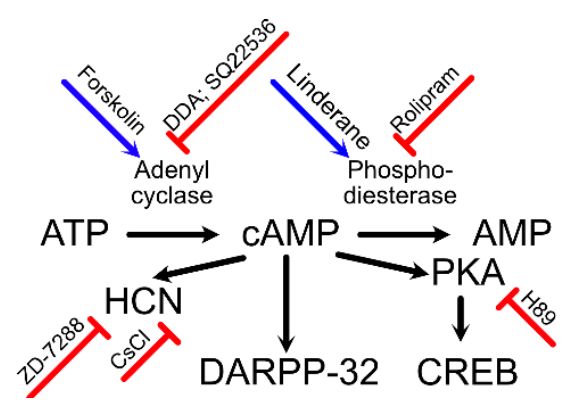

Figure 2. cAMP signaling.

Illustration of cAMP signaling and cAMP regulators. Black arrows and blue arrows = activation, red lines = inhibition, $\mathrm{ATP}=$ adenosine triphosphate, cAMP $=$ cyclic adenosine monophosphate, $\mathrm{AMP}=$ adenosine monophosphate, $\mathrm{HCN}=$ hyperpolarization activated cyclic nucleotide gated channel, PKA = protein kinase A, DARPP-32 = dopamine- and cAMP-regulated neuronal phosphoprotein, CREB $=$ cAMP response elementbinding protein.

Aside from NMDA receptors, cAMP also mediates the function of a variety of metabotropic neurotransmitter receptors. Examples of these include dopamine receptors, serotonin receptors and muscarinic acetylcholine receptors ${ }^{209-211}$. In the case of metabotropic receptors, the impact on cAMP is mediated by G proteins, which bind and activate PDE or AC enzymes ${ }^{212}$. This then leads to downstream signaling mediated by enzymes, such as PKA or DARPP-32 (Figure 2) ${ }^{210}$. 
A variety of methods can be used to measure the levels of cAMP in cells. For instance, genetically encoded cAMP sensors can be used, which are sensors that detect cAMP using cAMP-interacting domains from proteins such as PKA or EPAC ${ }^{213}$. For visualizing cAMP change, these sensors normally use fusion proteins incorporating the cAMP binding protein with a cyan fluorescent protein (CFP) and a yellow fluorescent protein (YFP). When cAMP increases in the presence of this sensor, the fusion protein changes its conformation, which in turn changes the ratio of CFP to YFP through a mechanism known as Förster resonance energy transfer (FRET) ${ }^{213}$. Examples of such cAMP FRET sensors include AKAR4-cAMPs and Epac1-cAMPs ${ }^{213,214}$. Aside from FRET sensors, other types of genetically encoded cAMP sensors also exist, such as the GFP-derived cAMPr ${ }^{215}$.

\subsubsection{Hyperpolarization-activated cyclic nucleotide-gated channels (HCN channels)}

One of the most interesting downstream targets of cAMP signaling are $\mathrm{HCN}$ channels, which are voltage-gated membrane channels that depolarize the cell upon opening and that have a higher conductivity at hyperpolarized membrane channel voltages. HCN channels are present in a variety of organs, including the central nervous system, where it fine-tunes the cellular membrane potential when it is near resting level $\mathbf{l}^{38,216}$.

As the name of the family implies, HCN channel activity can be regulated by cAMP, which binds the cyclic nucleotide binding domain of the protein. Doing this, the activation midpoint of the HCN channel is switched to be more positive, which leads to an increase of HCN channel conductance at membrane potential values near resting level ${ }^{217,218}$. Notably, the activation midpoint can also be affected by other mechanisms, including by binding the membrane-binding signaling molecule phospatidylinositol-4,5biphosphate $\left(\mathrm{PIP}_{2}\right)$, which has a similar impact on cAMP ${ }^{219,220}$.

HCN channels can be inhibited and probed with a variety of molecular factors. One approach involves targeting the cAMP levels by modulating the activity of AC or PDE enzymes. For AC regulation, forskolin can be used to activate the enzyme, whereas 2',3'-Dideoxyadenosine (DDA) and SQ-22536 can be used to inhibit the enzyme ${ }^{221-226}$. For PDE regulation, rolipram can be used to inhibit PDE4, whereas linderane can be used to indirectly activate PDE3 ${ }^{224,227}$.

$\mathrm{HCN}$ channels can also be probed with other molecular factors. For instance, $\mathrm{HCN}$ channels can be inhibited with a millimolar concentration of cesium ions, which is a pore blocker ${ }^{228-231}$. ZD-7288 can also be used to inhibit HCN channel activity by shifting its activation midpoint to a more negative value ${ }^{122,232}$. Finally, agents developed to affect HCN channels in heart tissue, such as zatebradine, ivabradine and cilobradine can be used as inhibitors also ${ }^{233,234}$.

$\mathrm{HCN}$ channels have a number of functions, one of which is involvement in the generation of network oscillations such as the thalamocortical delta waves. During generation, cells are depolarized by a current mediated by HCN channels, which depolarizes the membrane to the cell's threshold potential, which activates T-type calcium channels and thus leads to the generation of action potentials. After this, 
T-type calcium channels enter an inactive state, causing the cell to become hyperpolarized, which in turn causes the activity of $\mathrm{HCN}$ channels to increase, as they are more active at hyperpolarized membrane voltages. In reaction to this, the cell becomes more depolarized, leading the cell to again reach its threshold potential, thus repeating the cycle $38,235,236$.

HCN channels are also thought to be involved in the generation of spindle waves, which are a NREM sleep associated qEEG pattern involving an alteration between a 1-4 second period of depolarization, followed by a 5-20 second refractory period. These waves are generated similarly to thalamocortical delta waves, in that the depolarization requires hyperpolarization to activate the T-type calcium channel. In the case of spindle waves however, the depolarization causes the cell to accumulate calcium, activating a calcium-dependent adenyl cyclase and leading to cAMP production. cAMP binding then shifts the midpoint of HCN channels, causing a low-level depolarization of the cell that prevents the cell from becoming hyperpolarized. This state dissipates slowly and is responsible for the long refractory period that is characteristic of spindle waves ${ }^{237-239}$.

Finally, $\mathrm{HCN}$ channels are thought to be involved in the transition from spindle wave associated NREM sleep to wakefulness or REM sleep by acting as mediators of cAMP signaling. More specifically, in order to trigger this transition, the spindle wave pattern is abolished by a permanent increase of cAMP levels that is in turn triggered by an increase in neurotransmitter signaling 38,236 .

\subsection{3. cAMP and HCN channel disruptions in PD}

Notably, some studies have indicated a possible connection between cAMP misregulation and PD. For instance, studies in both PD models and patients have observed altered levels of adenylate cyclase activity, with both increases and decreases in activity being reported in different experimental contexts 4,34-36,212,240,241. In parallel to these results, alterations in cAMP levels have also been observed and some studies have also reported that cAMP-boosting small molecules can have a neuroprotective impact in PD models ${ }^{242-245}$. Finally, some limited data also exists from studies of PD patient CSF, which suggest that PD patients have reduced levels of cAMP - these studies however are dated and are contradicted by the results of other studies ${ }^{37,246}$

Notably, PD-related disruptions of HCN channels have also been observed. For instance, one study in 6-OHDA rats has reported that HCN-channel dependent pacemaking is reduced in the globus pallidus of these animals, which they postulate is a downstream result of dopamine depletion ${ }^{247}$. Similar reductions of $\mathrm{HCN}$ channel function in response to dopamine depletion have been observed in MitoPark rats, which are rats exhibiting disrupted mitochondria that are used in some studies as a PD model ${ }^{248}$. Finally, disruptions of hyperpolarization-activated inward rectifier current have also been observed in substantia nigra dopaminergic neurons in 1-methyl-4-phenylpyridinium (MPP+) toxicity models of $\mathrm{PD}^{249}$. 


\subsection{Aims of the project}

As mentioned, studies in both patients and test animals have observed that increased $\alpha$-synuclein levels lead to disruptions in rhythmic activity ${ }^{13,133}$. In this study, our intention was to investigate these disruptions, more specifically by identifying possible mechanisms and pathways that could explain why $\alpha$-synuclein changes the behavior of rhythmic activity. In order to investigate this, cortical neuron cultures were used, which allow for the application of several experimental modalities and also permit the analysis of extracellular $\alpha$-synuclein. With regard to specific experimental questions, our intention was to investigate the following:

1. Does $\alpha$-synuclein overexpression alter the rhythmic activity of cultured neurons?

2. How much do released $\alpha$-synuclein species play a role in regulating cultured neuron rhythmic activity?

3. Does $\alpha$-synuclein overexpression disrupt connecting structures, such as neurites and synapses?

4. Does $\alpha$-synuclein overexpression disrupt levels of cAMP, a signaling molecule that regulates synaptic potentiation and $\mathrm{HCN}$ channels?

5. Do other members of the synuclein family alter the rhythmic activity of cultured neurons when overexpressed?

6. Is in vitro rhythmic activity altered by overexpression of tau protein, a neurodegenerative disorder associated protein that is not a part of the synuclein family? 


\section{Materials and methods}

\subsection{Experimental materials and their preparation}

\subsubsection{Cultured neurons}

Cultured neurons analyzed in the experiments were harvested from embryonic day 18 (E18) embryos of Wistar rats, which are outbred experimental albino rats ${ }^{250,251}$. Notably, the age of the embryos was suitable for experiments of neuron network bursting, as various studies of this phenomenon in cultured neurons had used similarly aged embryos (e.g. E16, E18) ${ }^{86,118}$. For plating, cortices without hippocampi were harvested and the neurons were plated at a density of 250000 neurons per well ${ }^{84}$.

\subsubsection{Cultured neuron preparation}

Before preparation cell culture plates (Greiner) were coated with polyornithine (Sigma, $100 \mu \mathrm{g} / \mathrm{ml}$ ) at $4^{\circ} \mathrm{C}$ for at least one week. Following this, the plates were coated with laminin (Sigma, $1 \mu \mathrm{g} / \mathrm{ml}$ ) at $4^{\circ} \mathrm{C}$ for at least one day. On the day of plating, the laminin was extracted and the plates washed with phosphate-buffered saline (PBS). Following this, 2/3 of the final volume of the culture medium was added to the wells and the wells were incubated at $37{ }^{\circ} \mathrm{C}$ and $5 \% \mathrm{CO}_{2}$ until the neurons were ready to be plated.

Following this, pregnant rats were euthanized and their embryos extracted. Then, cortices without hippocampi were isolated from E18 rat embryos and collected into $4^{\circ} \mathrm{C}$ calcium magnesium free (CMF) medium, which was prepared by diluting Hank's balanced salt solution (HBSS) 1:9 in distilled water and adding sodium bicarbonate until 7.5\% (pH 7.2-7.4) ${ }^{84}$. The cortices were trypsinated with $0,25 \%$ trypsin (Sigma) for 15 minutes and treated with DNAse (Roche) for 10 minutes, following which trypsin was inhibited by addition of $4^{\circ} \mathrm{C}$ fetal calf serum (FCS, BioChrom). The cortices were then triturated, after which the debris was allowed to sediment without centrifugation. The supernatant from this was centrifuged, the liquid portion discarded and the cells resuspended in medium.

After resuspsension, the cells in the medium were counted using a counting chamber (Neubauer) and trypan blue to permit seeding at the desired density of 250000 neurons per well. The cells were then cultured in Neurobasal medium (Thermo Fisher Scientific), which had been supplemented with transferrin (AppliChem), penicillin (Thermo Fisher Scientific), streptomycin (Thermo Fisher Scientific), neomycin (Thermo Fisher Scientific), L-glutamine (Sigma) and 1x B27 supplement (Thermo Fisher Scientific).

Importantly, culture plates were plated in such a way that only 8 of the 24 wells of the Greiner plate were filled with neurons. More specifically, corner-wells were excluded and the non-corner wells along 
the long edge of the plate were plated. The inner wells were filled with either medium or PBS. This arrangement was performed to regularize evaporation in the culture plates.

\subsubsection{Cultured neuron preparation on coverslips}

For coverslips, $12 \mathrm{~mm}$ coverslips (Menzel) were incubated in $\mathrm{HCl}(1 \mathrm{M})$ at $20^{\circ} \mathrm{C}$ overnight. Following this, they were heated in a microwave at $180{ }^{\circ} \mathrm{C}$ for 4 hours for additional sterilization. The coverslips were then added to the plates, following which the plates were plated with neurons as normal.

\subsubsection{Cultured neuron incubation}

All neurons were incubated at $5 \% \mathrm{CO}_{2}$ and $37{ }^{\circ} \mathrm{C}$ inside a Labotect Labo $\mathrm{C} 101$ incubator for the duration of the culture's lifespan. Notably, the internal atmosphere of the incubator was hypersaturated with water vapor. For this, a large Petri dish filled with water was added to the bottom of the incubator. Additionally, the incubator itself also refreshed the water vapor atmosphere from a water supply of distilled water. The cultured neurons were specifically cultured in $750 \mu 1$ cultured medium, of which the culture medium lost 100-150 $\mu$ l through evaporation over the 4-5 week incubation period. The medium was also exchanged once on day in vitro 4 (DIV4).

\subsubsection{Overexpressed proteins and sensors}

A variety of overexpressed proteins were used over the course of our study. In all cases, these were overexpressed using AAV6 viral particles containing the cDNA of the protein, with neurons transduced with these particles on either DIV2 or DIV4. Notably, the expression of the cDNA was in all cases under the control of the neuron-specific promoter hSyn, ensuring neuron-specific expression.

\subsubsection{Bcl-xL - anti-apoptotic protein}

B-cell lymphoma extra large (Bcl-xL) is an anti-apoptotic protein localized inside the mitochondrial membrane, which inhibits the death of neurons by blocking cytochrome C release ${ }^{252}$. Notably, our group had previously used this protein to inhibit the death of neurons in cell culture ${ }^{113}$. This was done in this study as well to enable the study of neurons in the absence of apoptotic cell death.

For the expression of $\mathrm{Bcl}-\mathrm{xL}, 3 \times 10^{7}$ adeno-associated virus (AAV) viral particles containing a $\mathrm{Bcl}-\mathrm{xL}$ cDNA were pipetted on the neurons on DIV2. Notably, the Bcl-xL cDNA also included an AU1 antibody-binding tag, which was used to identify the produced Bcl-xL protein using Western Blot. $\mathrm{Bcl}-\mathrm{xL}$ was overexpressed in all cultures unless mentioned otherwise.

\subsubsection{Synucleins $-\alpha$-synuclein, $\beta$-synuclein, $\gamma$-synuclein, $\beta$-synuclein mutants}

In this study, three different wild-type synucleins were tested - wild-type $\alpha$-synuclein, $\beta$-synuclein and $\gamma$-synuclein. Alongside these, two mutant synucleins were tested - the V70M $\beta$-synuclein mutant and the $\mathrm{P} 123 \mathrm{H} \beta$-synuclein mutant. The properties of these proteins have been described more in the introductory section of this study. Briefly, $\alpha$-synuclein and the $\beta$-synuclein mutants are disease- 
associated aggregating proteins, which have been associated with diseases known as synucleinopathies ${ }^{65,83,84}$. Meanwhile, wild-type $\beta$-synuclein is claimed by some studies to also have a neurotoxic impact, whereas wild-type $\gamma$-synuclein is generally thought to be the least toxic of the synuclein variants due to the low amount of data suggesting it has a neurotoxic role ${ }^{78}$. Notably, $\gamma$-synuclein was the main control used in our study due to its close structural relationship with the other synucleins as well as due to its relative non-toxicity.

For the expression of synuclein variants, expression using AAV viral particles after transduction on DIV4 was used. However, the amount of viral particles varied, with most experiments using $2 \times 10^{8}$ AAV viral particles of synuclein-overexpressing virus. This viral titer was selected, as viral titers higher than $2 \times 10^{8}$ caused an impact on neuronal functioning due to the viral load used (Figure 3 ).
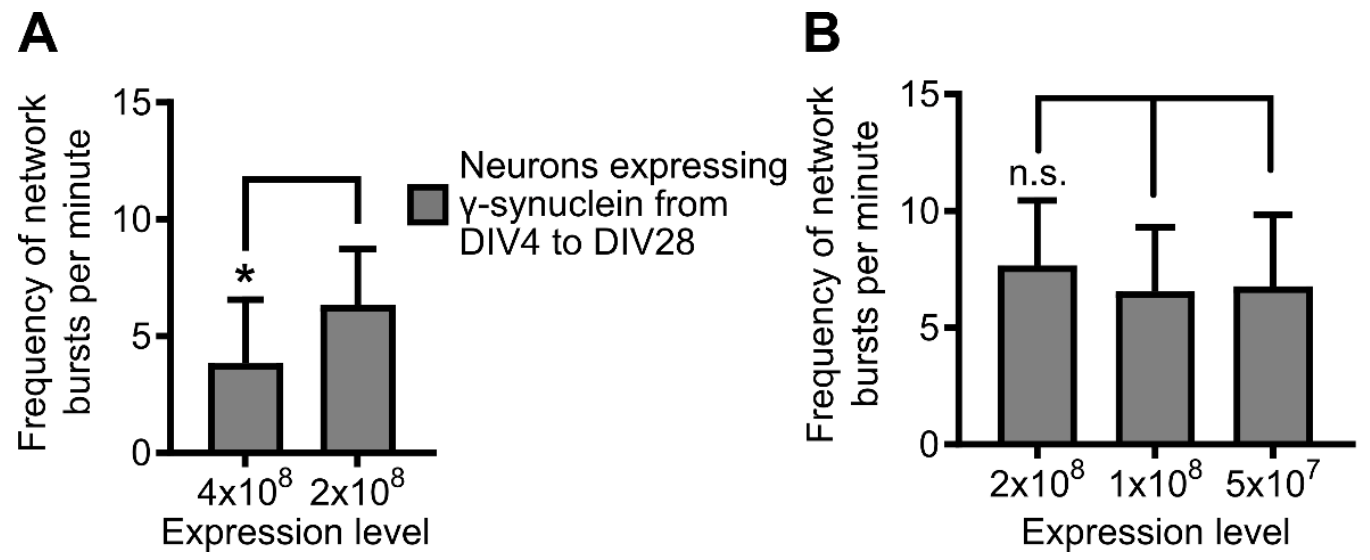

Figure 3. Inducing expression using $4 \times 10^{8} \mathrm{AAV}$ viral particles reduces the frequency of network bursting, whereas inducing expression with $2 \times 10^{8} \mathrm{AAV}$ viral particles does not.

(A-B) Quantification of the frequency of network bursts in the presence of different overexpression levels of the control protein $\gamma$-synuclein. Overexpression levels are denoted by the viral titer used to induced the expression, with the number indicating the amount of transducing viral particles. $2 \times 10^{8}$ viral particles is the same overexpression level as used in Figure 18 and this is compared with either $4 \times 10^{8}(\mathbf{A})$ or with $1 \times 10^{8}$ and $5 \times 10^{7}$ viral particles (B). Statistical analysis: a two-tailed t-test with Welch's correction (A) or a one-way ANOVA (B). Statistical power was determined with a post-hoc test. "n.s." = not significant. *: $\mathrm{p}<0.05$. Statistical power: $1-\beta$ $>63 \%$ (A), sample size: 12 independent cultures (A), 9 independent cultures (B).

Importantly, two sets of viral particles were used for testing the synucleins. The first set included a complete set of wild-type synucleins. In this set, all of the viral vectors also simultaneously overexpressed nuclear localization signal tagged mCherry (NLS-mCherry), a red fluorescent protein expressed in the nucleus. This set was used for all analyses involving wild-type synucleins.

In comparison, the second set was made up of viruses not overexpressing NLS-mCherry but only synucleins. As with the first set, the set was complete, including all wild-type synucleins as well as the $\beta$-synuclein mutants. This set was used for all analyses involving the $\beta$-synuclein mutants. A different set was used for this analysis as it was the same set that was used to analyze these proteins in a dedicated study of $\beta$-synuclein mutants prepared in our lab by Dr. Maryna Psol ${ }^{84}$. 


\subsubsection{Tau protein}

Tau protein is a cytoskeletal protein involved in regulating cytoskeletal stability, which has been implicated in Alzheimer's disease due to it being a component of neurofibrillary tangles ${ }^{253,254}$. In our study, it was used as a point of comparison for the synucleins. More specifically, if synucleins were observed to interfere with neuron functioning, the impact of tau protein was also determined to identify if the observed impacts were synuclein specific.

For the overexpression of tau protein, a similar protocol was used as for synuclein overexpression. On DIV4, $2 \times 10^{8}$ AAV viral particles were used to transduce the neurons to induce tau protein overexpression. Notably, this viral vector also expressed NLS-mCherry alongside tau protein from the same viral vector genome.

\subsubsection{GCaMP6 - calcium sensor}

GCaMP6 is a genetically-encoded calcium sensor localized in the cytosol that is used to detect calcium changes inside cells ${ }^{255,256}$. Structurally, it is composed of a green fluorescent protein (GFP) alongside a calmodulin domain and an M13 peptide sequence from a myosin light chain kinase. Upon calciumbinding, an interaction between the calcium ions, calmodulin and the M13 peptide sequence causes a conformation change of the GFP protein, increasing its fluorescence (Figure 4).

In this study, GCaMP6 was used to indirectly measure network bursts, which take the form of neuron network spanning depolarizations. This was possible, as a calcium influx is known to follow the depolarization, which can be detected using the calcium sensor. Indeed, network bursts are easily measurable using this setup and they show up as a spike in the calcium sensor fluorescence in several neurons at the same second (Figure 4). For expression of GCaMP6, neurons were transduced on DIV2 with $3 \times 10^{7}$ GCaMP6-encoding AAV viral particles.

A
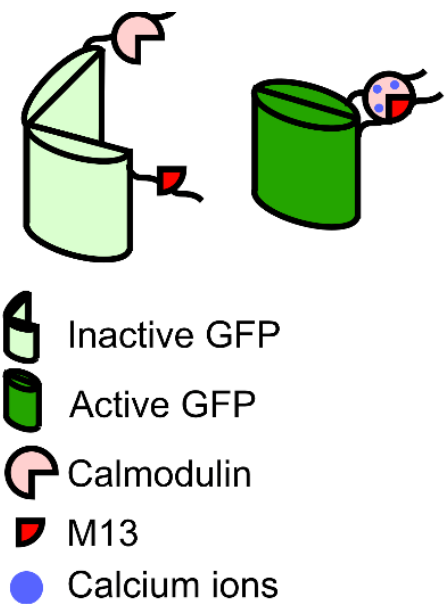

B

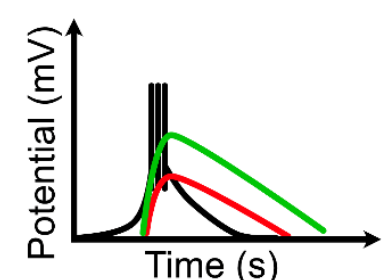

入otential change

$\sim$ Calcium influx

Fluorescence change
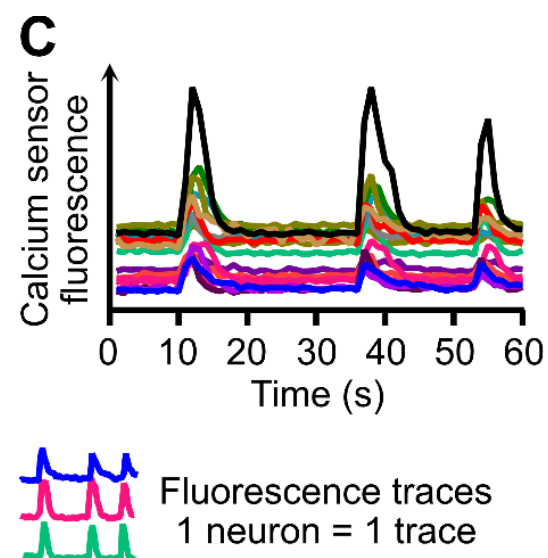

Fluorescence traces 1 neuron $=1$ trace

Figure 4. GCaMP increases in fluorescence in response to a calcium influx, such as those observed in neurons during network bursting.

(A) Illustration of the GCaMP fluorescence increase in response to calcium ions. Depicted are inactive GCaMP6 with low fluorescence (left image) and active GCaMP6 with increased fluorescence (right image).

(B) Illustration of the relationship between depolarizations, the calcium influx and the fluorescence change.

(C) Example calcium sensor fluorescence traces of neurons recorded over 60 seconds. 


\subsubsection{NLS-mCherry - nucleus-marking fluorophore}

mCherry is a red fluorescent protein derived from DsRed, which in this study was used in combination with a nuclear localization signal (NLS) for the easy quantification and identification of neurons ${ }^{257}$. This was made possible due to the simple, evenly shaped fluorescent signal that is produced by neuronal nuclei expressing this fluorophore, which in turn made it easy to identify them using an automated computer analysis procedure.

In this study, NLS-mCherry was expressed alongside either synucleins or tau protein. This was accomplished as the viral vectors used for tau protein or synuclein overexpression also contained a complementary DNA (cDNA) sequence that induced NLS-mCherry expression. In some experiments, NLS-mCherry was also expressed using a virus that only encoded NLS-mCherry. In either case, neurons were transduced with $2 \times 10^{8}$ viral particles to induce NLS-mCherry expression on DIV4.

\subsubsection{Sy-GCaMP - pre-synaptically located calcium sensor}

Synaptophysin-GCaMP (Sy-GCaMP) is a variant of the GCaMP3.5 calcium sensor, which contains a synaptophysin anchor designed to localize the GCaMP3.5 inside the presynapse ${ }^{255}$. In this study, this sensor was used to quantify presynapses using a dynamic method. More specifically, our study counted only those synapses identified to take up calcium using Sy-GCaMP, thus excluding the quantification of inactive synapses and debris (Figure 5). In this study, Sy-GCaMP was expressed by transducing neurons on DIV2 with $3 \times 10^{7}$ AAV viral particles encoding this sensor.
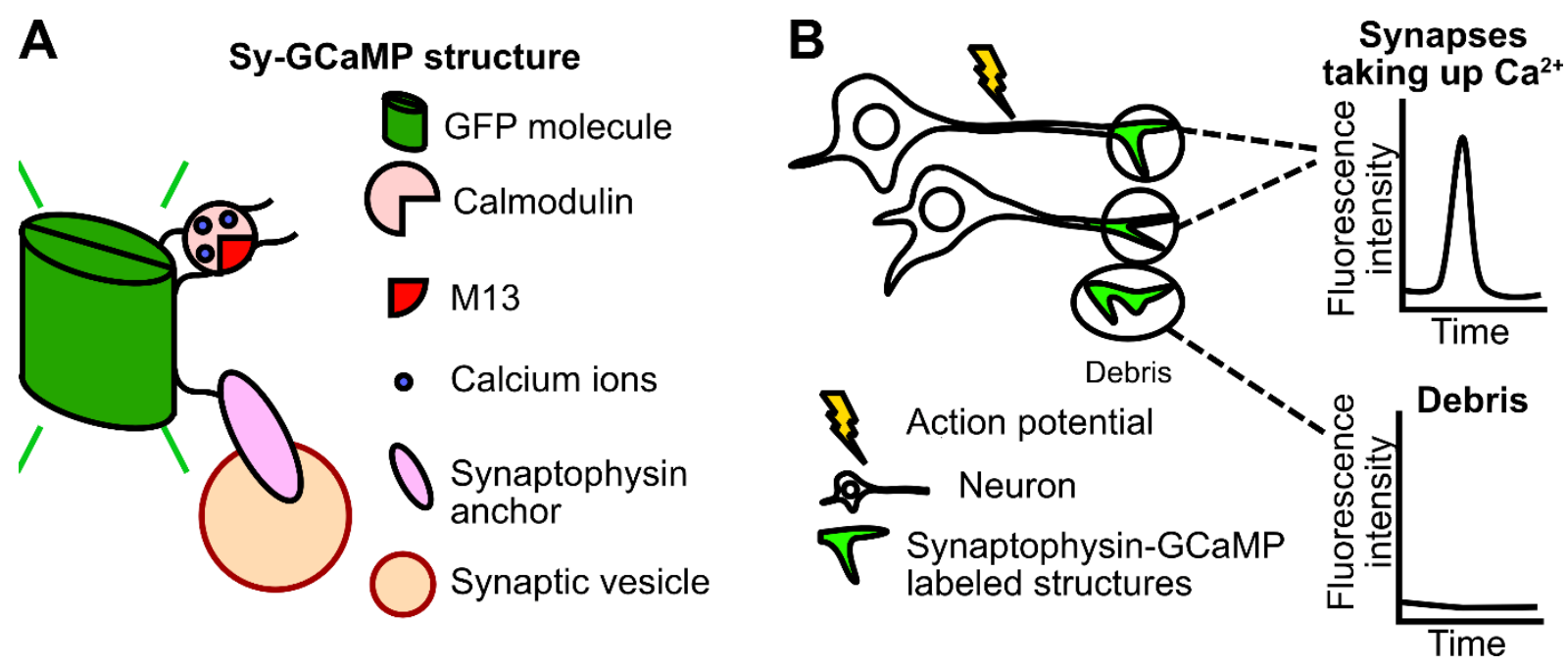

Figure 5. Synaptophysin-GCaMP is localized in synapses and provides a dynamic signal that responds to changes of calcium levels at the pre-synapse.

(A) Illustration of the Sy-GCaMP structure and its function. Sy-GcaMP = synaptophysin-GCaMP, GFP = green fluorescent protein, M13 = M13 peptide sequence.

(B) Illustration of the method used to differentiate between active synapses and debris. The graphs indicate the change of calcium sensor fluorescent intensity over time. Spikes in fluorescence intensity over time were considered to characterize active synapses, whereas flat fluorescence intensity was considered to characterize inactive synapses and debris. 


\subsubsection{7. cAMPr - cAMP sensor}

cAMPr is a cAMP sensor that contains a green fluorescent protein (GFP) as the fluorescent component, as well as two domains derived from the cAMP-binding protein kinase PKA - PKA-C and PKA-R. In the resting state, the PKA-derived domains are bound to each other. However, upon cAMP introduction, the interaction is broken and the fluorescence of cAMPr increases (Figure 6). In this study, cAMPr expression was induced by transducing neurons on DIV4 with $1 \times 10^{8}$ viral particles.

A
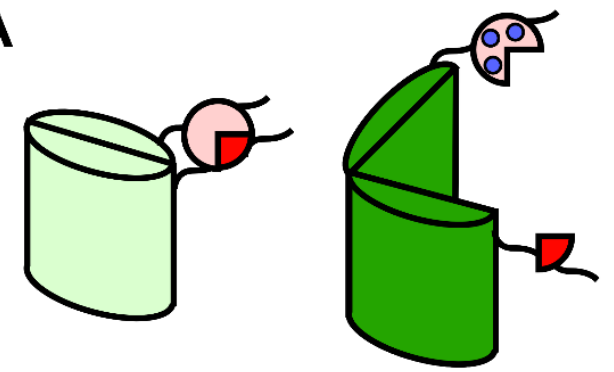

Inactive GFP
Active GFP

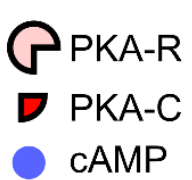

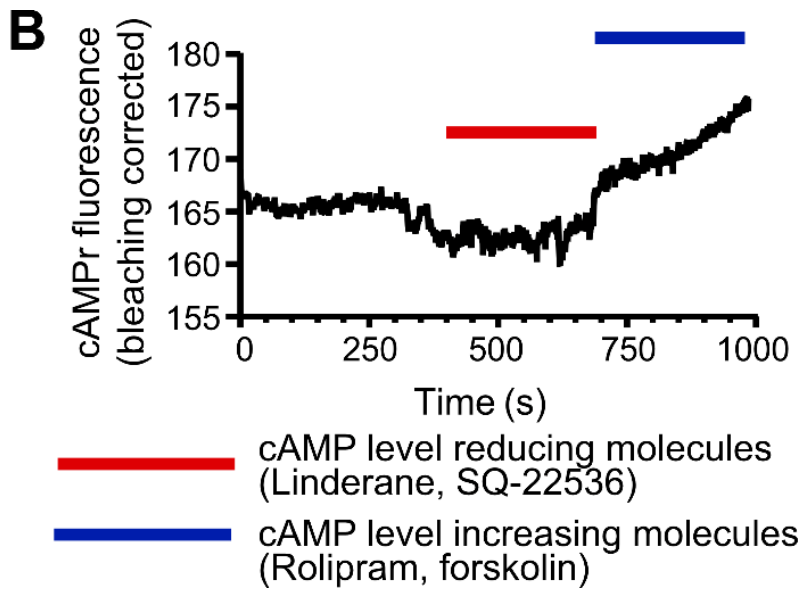

Figure 6. The cAMPr sensor directly measures cAMP level using GFP and PKA domains ${ }^{215}$.

(A) Illustration of the cAMPr mechanism during resting state (left panel) and after cAMP binding (right panel). $\mathrm{GFP}=$ green fluorescent protein, $\mathrm{PKA}-\mathrm{R}=$ regulatory subunit of PKA, PKA-C = catalytic subunit of PKA, cAMP = cyclic adenosine monophosphate, $\mathrm{PKA}=$ protein kinase $\mathrm{A}$.

(B) Sample cAMPr trace of a neuron expressing cAMPr, which is treated with cAMP level changing molecules. More specifically, a $\gamma$-synuclein overexpressing neuron was imaged on DIV15 for the generation of this image. The trace has been corrected for bleaching. Red line = time duration for applying cAMP level reducing molecules (linderane $20 \mu \mathrm{M}$, SQ-22536 $20 \mu \mathrm{M}$ ), blue line = time duration for applying cAMP level increasing molecules (rolipram $5 \mu \mathrm{M}$, forskolin $5 \mu \mathrm{M}$ ).

\subsubsection{AKAR4-cAMPs - sensor of phosphorylation by PKA, a cAMP regulated kinase}

AKAR4-cAMPs is a sensor that detects phosphorylation by protein kinase A, which is a cAMP-activated protein kinase. With regard to its components, it consists of a cyan fluorescent protein (CFP), a yellow fluorescent protein (YFP), a SUB domain and a PABD domain. In terms of its design, AKAR4-cAMPs functions as a Förster resonance energy transfer (FRET) sensor. In the resting state, the two fluorophores are separated in space, meaning that an excitation of the CFP fluorophore will trigger light emission primarily at the wavelength characteristic of CFP. After the phosphorylation of the SUB domain however, the conformation of the protein changes in such a way as to move the fluorophores closer to each other in space. As a result, excitation of the CFP fluorophore will trigger light emission also at the wavelength characteristic of YFP (Figure 7).

In this study, AKAR4-cAMPs was primarily used as a cAMP sensor. This was possible, as the signal is responsive to cAMP increases due to the close relationship between cAMP levels and PKA phosphorylation. Indeed, this close relationship was visible when using the sensor, as the AKAR-cAMPs sensor signal was responsive to small molecules that increase or decrease cAMP levels (Figure 7). In 
this study, AKAR4-cAMPs was expressed in the neurons by transducing them on DIV4 with $1 \times 10^{8}$ viral particles encoding the protein.

A

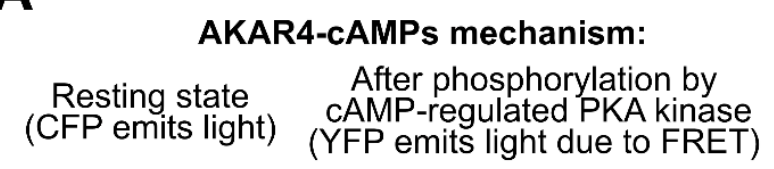

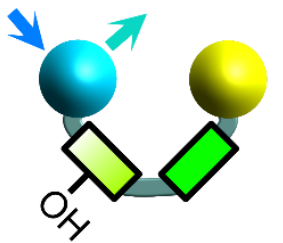

CFP

YFP

SUB domain

PABD domain
B

AKAR4-cAMPs response to cAMP regulators

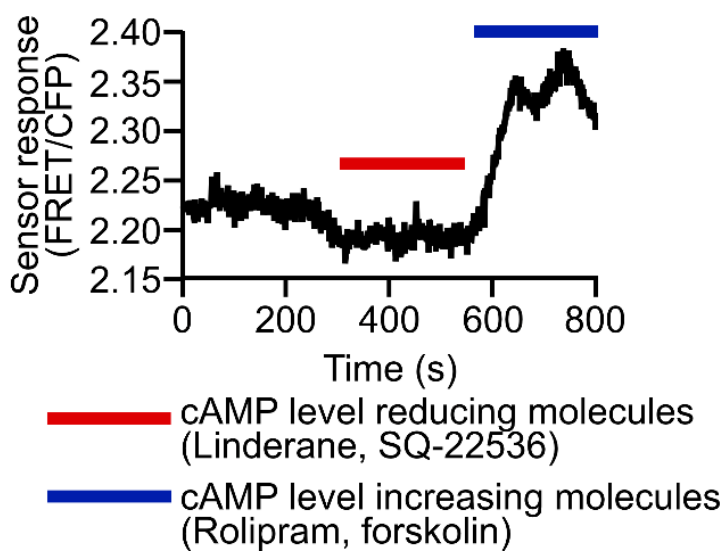

Figure 7. AKAR4-cAMPs sensor is a FRET sensor responsive to PKA phosphorylation, which itself is responsive to cAMP levels ${ }^{214}$.

(A) Illustration of the AKAR4-cAMPs mechanism in the resting state (left) and after PKA phosphorylation (right). "-OH" = free hydroxyl group of the SUB domain. "-OP" = phosphorylated hydroxyl group of the SUB domain. "CFP" = cyan fluorescent protein. "YFP" = yellow fluorescent protein. "FRET" = Förster resonance energy transfer. "PKA" = Protein kinase A. "cAMP" = cyclic adenosine monophosphate.

(B) Sample FRET/CFP trace of a neuron expressing AKAR4-cAMPs, which is treated with cAMP level changing molecules. The graph illustrates the change of the cAMP-sensitive FRET/CFP ratio of the sensor. Red line $=$ time duration for applying cAMP level reducing molecules (linderane $20 \mu \mathrm{M}$, SQ-22536 $20 \mu \mathrm{M}$ ), blue line $=$ time duration for applying cAMP level increasing molecules (rolipram $5 \mu \mathrm{M}$, forskolin $5 \mu \mathrm{M}$ ).

\subsubsection{Viral vectors and particles}

\subsubsection{List of viral vectors}

For protein expression, our study used AAV6 viral particles containing AAV vectors. For preparing these vectors, the same basic design was used. On the ends of these vectors, inverted terminal repeats (ITRs) were included, which are required for virus packaging. Each cDNA expressed from the vector was placed under the control of a human synapsin promoter (hSyn), which ensured neuron specificity. Downstream of the cDNA, most vectors also included a woodchuck hepatitis virus post-transcriptional regulatory element (WPRE) and a bovine growth hormone polyadenylation sequence (bGH pA), which were included to increase the level of cDNA expression.

Beyond this basic design, two subtypes of vectors could also be identified. The vectors used for overexpression of analytical sensors and $\mathrm{Bcl}-\mathrm{xL}$ were monocistronic, containing the cDNA of only one protein. By contrast, the vectors used for overexpression of tau protein and the synucleins were bicistronic, containing the cDNA for the protein and additionally NLS-mCherry. Importantly, the bicistronic vectors contained additional vector elements. These included the simian virus 40 polyadenylation site (SV40), used for polyadenylation, an intronic sequence (Int) and a transcription blocker (TB), used for stopping transcription at the blocker. 
The various viral vectors used are detailed more in Figure 8. Note that the bicistronic synuclein/tau protein expression viruses were used for the analysis of all wild-type synucleins and for the analysis of tau protein. By contrast, the monocistronic synuclein expression viruses were used for the analysis of only the $\beta$-synuclein mutants.

The majority of these viral vectors had been prepared in our lab over the course of previous studies. However, the cAMPr and AKAR4-cAMPs viral vectors were prepared during this study. Descriptions of their production have been included in order to demonstrate the basic concepts of molecular cloning applied for viral vector preparation.

A

Bicistronic expression virus set (wild-type Synuclein, tau protein)

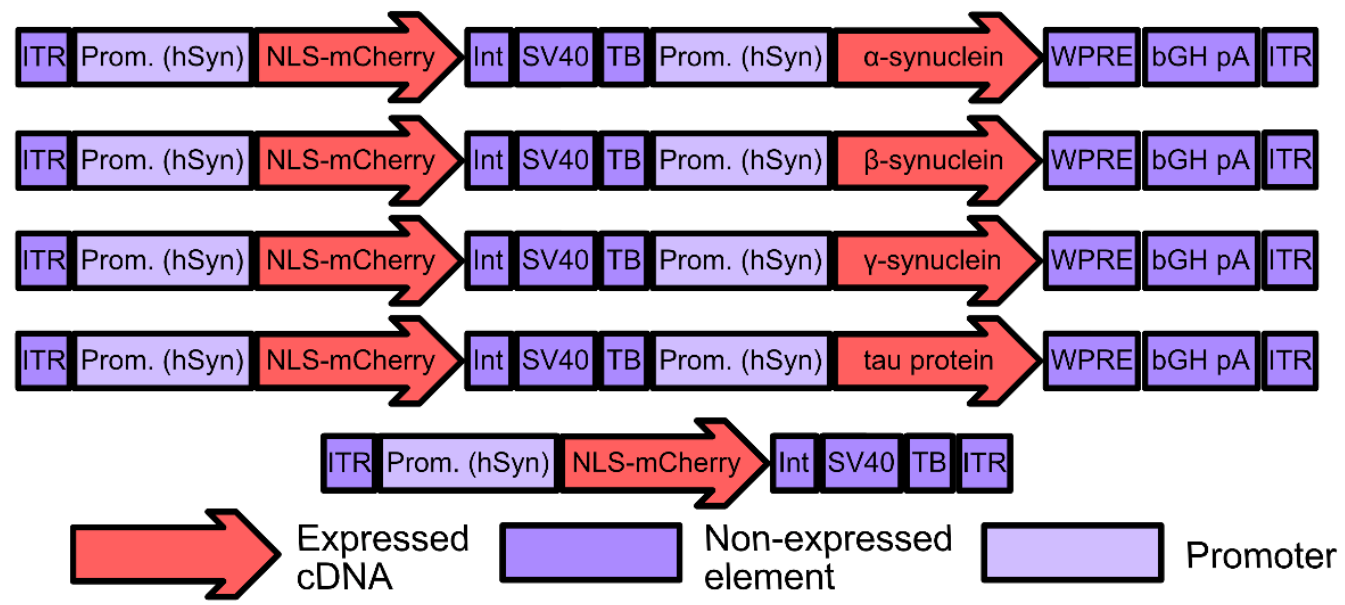

B

Monocistronic expression virus set (for $\beta$-synuclein mutant analysis only)
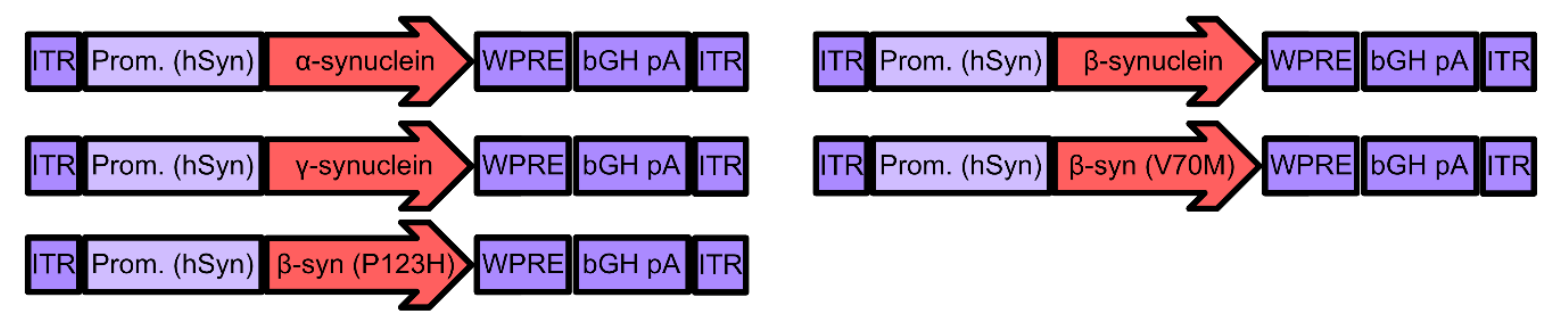

C

\section{Sensor viruses}
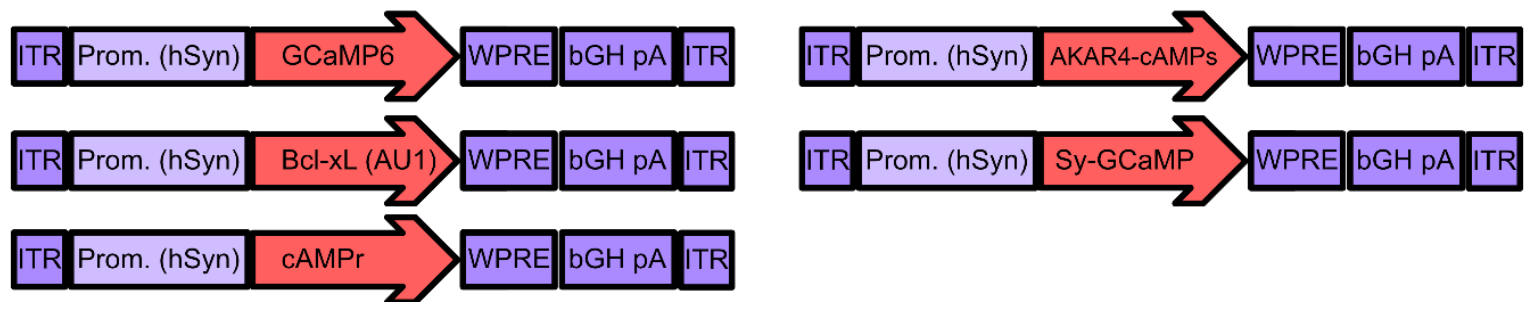

Figure 8. AAV6 viral vectors used during the study.

Illustrations of the various AAV overexpression vectors used in this study. Wide red arrows = expressed cDNAs, blue boxes $=$ non-expressed elements, light blue boxes $=$ gene promoters, hSyn $=$ human synapsin, $\mathrm{cDNA}=$ complementary cDNA, NLS-mCherry = nuclear localization signal tagged mCherry, WPRE $=$ woodchuck hepatitis virus post-transcriptional regulatory element, $\mathrm{bGH}$ polyA = bovine growth hormone polyadenylation sequence, $\mathrm{SV} 40=$ simian virus 40 polyadenylation site, $\mathrm{Int}=$ intronic sequence, $\mathrm{TB}=$ transcription blocker, $\beta$-syn $=\beta$-synuclein, ITR $=$ inverted terminal repeat, Sy-GCaMP $=$ synaptophysin-GCaMP. 


\subsubsection{Preparation of viral vectors using molecular cloning - cAMPr}

Cloning of the cAMPr AAV6 expression vector was performed in two stages. First, the sequence of interest was ordered, amplified and then cloned into an intermediary pMA vector. Second, the sequence was cloned into a pAAV vector without destroying the sensitive pAAV ITR sequences, required for virus production. The amplicon was cloned twice because direct cloning of the amplicon into pAAV vectors has a very low chance of success. This is because of the special conditions required to transform and grow AAV viruses, which are required for the preservation of its ITR sequences.

For the first step, bacteria containing the p2lox-cAMPr plasmid were ordered from Addgene, which expressed the single-wavelength fluorescent cAMP sensor $\mathrm{cAMPr}{ }^{215}$. Two primers were ordered from Sigma - cAMPr sense and cAMPr antisense (Table 1). These primers were designed for molecular cloning. More specifically, after production of the amplicon using the primers, the intention was to cut it with restrictases, producing specific sticky ends, following which the resulting fragment could be used to clone first into a pMA vector and then into a pAAV vector.

The primers were designed to have several components. The first component was a hexanucleotide sequence $\left(\mathrm{A}_{6}\right)$, which was selected to prevent disruption of the cloning site from nucleotide loss. The second component was a cloning site, which was selected to enable molecular cloning of the constructs. The third component was a non-coding segment from the AAV target plasmid. This was included to ensure that the resulting AAV plasmid would contain the same non-coding sequences as other AAV plasmids. Following this, the final component matched the cAMPr cDNA sequence, which was included to be able to hybridize the primer with the cAMPr target sequence and to be able to amplify it.

\begin{tabular}{|l|l|}
\hline Name & Primer sequence \\
\hline cAMPr sense & $\begin{array}{l}\text { AAAAAAGCTAGCAAGGATCCACCGGTCGCCACCATGGGCAACGC } \\
\text { CGCCGCCGCCAAGAA }\end{array}$ \\
\hline cAMPr antisense & AAAAAAAAGCTTGCGGCCGCTTTACATCTTCCGCTTTCTCAG \\
\hline AKAR4 sense & $\begin{array}{l}\text { AAAAAAGAATTCAAGCTGCTAGCAAGGATCCACCGGTCGCCACC } \\
\text { ATGGTGCGGGGTTCTCATCATCATCATCATCATGGTA }\end{array}$ \\
\hline AKAR4 antisense & AAAAAAAAGCTTGCGGCCGCTTTACTCGATGTTGTGGCGGAT \\
\hline
\end{tabular}

Table 1. Primers used for cloning AAV vectors.

After the arrival of the bacteria containing the p2Lox-cAMPr plasmid, the vector was extracted from the ordered bacteria using a QIAPrep Spin Miniprep Kit (Qiagen) according to manufacturer protocol. Following this, the plasmid DNA was further purified by sedimentation. For this, a tenth of the total mixture volume of $3 \mathrm{M}$ sodium acetate was added to the mixture. After acetate addition, $100 \%$ ethanol was added, the mixture stirred and stored at $-80{ }^{\circ} \mathrm{C}$ for five minutes. The mixture was then centrifuged at $13000 \mathrm{rpm}$ and $4{ }^{\circ} \mathrm{C}$ for 30 minutes, the ethanol supernatant was removed and $70 \%$ ethanol was added without mixing the pellet. The mixture was then centrifuged at $13000 \mathrm{rpm}$ and $4{ }^{\circ} \mathrm{C}$ for three minutes. 
For taking up DNA, the ethanol was removed, the DNA briefly dried and then dissolved in Trisethylenediaminetetraacetic acid (TE) buffer.

After DNA purification, the produced DNA was analyzed. For this, the concentration of the DNA was measured using a biophotometer (Eppendorf) and the identity of the DNA was confirmed using an analytical restriction. For determining the identity of the DNA, it was restricted in 1 x CutSmart buffer for 1 hour using NheI and HindIII restrictases. After restriction, the DNA fragments were analyzed on a $0.8 \%$ agarose gel containing ethidium bromide. The size of the resulting fragments was compared to fragments predicted using an in silico analysis. For electrophoresis, the E835 electrophoresis device (Consort) was used, whereas for gel visualization, the Gel Doc EQ (Bio-Rad) was used.

After the identity of the vector was confirmed, $20 \mathrm{pg} / \mathrm{l}$ of the p2Lox-cAMPr was used as a template for PCR amplification using the cAMPr sense and antisense primers. This was done to extract the cAMPr sequence so it could then be cloned into a pMA vector. For amplification, the MiniCycler PTC 150 (MJ Research) was used. Briefly, the templates were cycled 25 times with a PCR program, which consisted of a denaturation step at $98^{\circ} \mathrm{C}$ for 30 seconds, an annealing step at $60^{\circ} \mathrm{C}$ for 30 seconds and an elongation step at $72{ }^{\circ} \mathrm{C}$ for two minutes. The final elongation cycle was extended by an extra three minutes. Aside from the template, the PCR mixture contained $1 \mathrm{x}$ HF Phusion Buffer, $5 \mu \mathrm{M}$ cAMPr sense and antisense primer, $200 \mu \mathrm{M}$ deoxynucleotide triphosphates (dNTPs), $3 \%$ dimethyl sulfoxide (DMSO), $1 \mathrm{mM} \mathrm{MgCl} 2$ and $1 \mathrm{U} / \mu$ l Phusion DNA polymerase.

After the amplification was completed, the intention was to extract the amplicon using an agarose gel, following which it was to be cut for cloning into a pMA vector. In order to achieve the agarose gel extraction, the PCR mixture containing the amplicon was electrophoresed on an agarose gel using a Gel Doc EQ (Bio-Rad) chamber. After this, the band corresponding to the amplicon was cut out using a clean scalpel and then purified from the gel using the QIAquick Gel Extraction Kit according to manufacturer protocol. For this, briefly, the gel slice was dissolved, the DNA bound to the QIAquick column, washed and then precipitated. Following this, the DNA was precipitated using sodium acetate and ethanol, washed and then dissolved in TE.

After precipitation, the amplicon was cut using the NheI and HindIII restrictases for one hour in $1 \mathrm{x}$ CutSmart buffer, following which the mixture was electrophoresed again on an agarose gel, the larger fragment extracted using a scalpel and purified using the QIAquick Gel Extraction Kit. Simultaneously, the pMA-RQ-SolFscv backbone vector was cut using the NheI and HindIII restrictases for one hour in 1 x CutSmart buffer. This DNA was also electrophoresed on an agarose gel, the backbone fragment cut out using a scalpel and extracted using a QIAquick Gel Extraction Kit.

After the fragments were extracted, they were washed by sedimentation with ethanol in the presence of sodium acetate and dissolved in TE. Their concentration was then determined. After this, these fragments were ligated together using a T4 DNA Ligase in $1 \mathrm{x}$ T4 Ligase Buffer, yielding a pMA vector 
containing a cAMPr sequence. For the ligation, briefly, the DNA was heated at $45^{\circ} \mathrm{C}$ for 5 minutes, after which the ligase and buffer was added, then ligation was performed at $16{ }^{\circ} \mathrm{C}$ for 15 minutes and subsequently $25^{\circ} \mathrm{C}$ for 15 minutes. For the ligation a molar ratio of 3:1 (insert to vector) was used.

After the pMA-cAMPr vector had been ligated, the intention was to transform it into bacteria so it could be multiplied before cloning into the pAAV vector backbone. For transformation, $50 \mu \mathrm{l}$ chemically competent 5- $\alpha$ Escherichia coli bacteria were used according to manufacturer protocol. Briefly, the bacteria were thawed and mixed with DNA. The mixture was gently flicked and cooled on ice for 30 minutes. Following this, a $42^{\circ} \mathrm{C}$ heat shock was applied to the bacteria for 30 seconds using a water bath. Following this, the mixture was cooled on ice for 5 minutes, then mixed with SOC medium and shaken at $180 \mathrm{rpm}$ and $37^{\circ} \mathrm{C}$ for 1 hour. Following this, the bacteria were smeared on LB agar plates containing an ampicillin antibiotic and incubated overnight at $37{ }^{\circ} \mathrm{C}$. On the following day, bacterial colonies were seeded into liquid LB medium containing an ampicillin antibiotic.

After transformation and bacterial incubation, the resulting pMA-cAMPr vectors were harvested from the bacteria. For this, DNA was purified from the bacterial preparations using a QIAprep Spin Miniprep Kit and then precipitated. The identity of the plasmid contained in the colony was confirmed using an analytical restriction, which utilized the BamHI restrictase in a 1 x CutSmart buffer. Following the restriction, DNA samples were sent for an exact sequence confirmation to the MicroSynth SeqLab.

After the pMA-cAMPr vectors were prepared, it was possible to finally perform cloning into the pAAV vector. Notably, all of the preliminary steps were taken for two reasons. Firstly, it was desired to fully confirm the sequence of the amplicon using sequencing before it was cloned into the pAAV vector. This was done to prevent wasting resources on the cloning and preparation of faulty pAAV vectors. Secondly, it was also desired to optimize the conditions for pAAV vector ligation, which requires high concentration and pure DNA fragments, such as those that can be acquired from the restriction of a plasmid.

In order to clone the cAMPr sequence into the pAAV vector, the pAAV backbone and the pMA intermediary vector were restricted and the fragments were then ligated. For the restriction, pMA cAMPr vector was cut using the NheI and HindIII restrictases in $1 \times$ CutSmart buffer. Simultaneously, the pAAV backbone vector was also cut using the NheI and HindIII restrictases in 1 x CutSmart buffer. Following the restriction, the mixtures were electrophoresed on an agarose gel, the fragments of interest were cut out using a scalpel and purified using a QIAquick Gel Extraction Kit. The fragments were further purified by being precipitated with sodium acetate and ethanol and then taken up in TE.

Following this, the concentration of the fragments was determined using an electrophotometer. Subsequently, the fragments were ligated using T4 DNA ligase in $1 \times$ T4 Ligase Buffer. Briefly, the DNA was heated at $45^{\circ} \mathrm{C}$ for 5 minutes, ligation was performed at $16^{\circ} \mathrm{C}$ for 15 minutes and subsequently at $25{ }^{\circ} \mathrm{C}$ for 15 minutes. For the ligation, a molar ratio of 3:1 (insert:vector) was used. 
After the pAAV-cAMPr constructs had been ligated, it was then necessary to transform these into bacteria. For this, it was required to use SURE electrocompetent bacteria instead of the 5- $\alpha$ bacteria used for normal transformation. This was done to ensure the survival of the ITR sequences of the ligated pAAV vectors, which are degraded inside $5-\alpha$ bacteria.

For the transformation, the SURE cells were placed on ice for ten minutes. Following this, DNA was added to the cells and gently stirred, then incubated on ice for one minute. After incubation, the bacteria were pipetted into a cuvette, dried and pulsed using $1.7 \mathrm{kV}$ and a GenePulser device. The cells were then incubated at $37{ }^{\circ} \mathrm{C}$ in SOC medium while being shaken at $180 \mathrm{rpm}$. The bacteria were spread on LB agar plates with the ampicillin antibiotic, which were incubated overnight at $37^{\circ} \mathrm{C}$.

On the next day, bacterial colonies were seeded into liquid LB agar medium. Following this, plasmids from the colonies were purified from the bacteria using the QIAprep Spin Miniprep Kit. The plasmids were then purified using precipitation and taken up in TE. The identity of the plasmids was confirmed using analytical restriction. For this, plasmids were cut in 1 x CutSmart buffer with the SmaI restrictase for 1 hour. The resulting fragments were electrophoresed on an agarose gel and the size of the fragments compared to the size of the predicted fragments. The colonies containing the plasmids of interests were kept at $4{ }^{\circ} \mathrm{C}$ for storage, following which they were used to seed a larger volume of liquid LB medium. These bacteria were grown overnight.

Following the incubation, plasmids were extracted from bacteria using the Nucleobond PC 2000 (Macherey-Nagel) kit according to manufacturer protocol. Briefly, bacteria were centrifuged and resuspended in an RNase a mixture. Following this, the bacteria were lysed and the bacterial mass suspended. The bacteria were suspended on ice and the supernatant transferred to a Nucleobond column. After binding of DNA, the column was washed and then eluted. The DNA mixture was precipitated and pelleted. Finally, the pellet was dissolved in TE. Following DNA dissolution, an analytical restriction was carried out using SmaI in 1 x CutSmart buffer to double check the identity of the plasmids. This restriction also served the secondary advantage of enabling us to determine the integrity of the ITRs. Following verification, the DNA was ready for viral preparation.

\subsubsection{Preparation of viral vectors using molecular cloning - AKAR4-cAMPs}

Preparation of the AKAR4-cAMPs AAV6 expression vector was largely similar to the procedure used for the cloning of the cAMPr AAV6 expression vector. As a result, only the unique portions of the protocol will be described.

With regard to the plasmids used as the source for the AKAR4 sequence, a pcDNA3-AKAR4 was used after being ordered from Addgene ${ }^{214}$. The primers used for amplification were AKAR4 sense and AKAR4 antisense (Table 1) For cloning sites, instead of the cloning sites used for the cAMPr vector, the AKAR construct amplicons were designed to use the EcoRI and HindIII cloning sites instead. 
As the most crucial differentiating step, the intermediary AKAR4 vector (pMA-AKAR4) was transformed twice. This was done as immediate transformation into the pAAV vector using SURE cells was impossible and yielded mutated dimeric DNA, likely due to the presence of additional repeat sequences (the homologous CFP and YFP cDNA sequences of the AKAR4-cAMPs cDNA). As a result, the bacteria were first transformed into XL-10 gold bacteria, which degraded the ITR sequences only in some of the transformed plasmids. As a result, it was possible to harvest the plasmids from the XL-10 gold bacteria (Stratagene) and then transform the ready-made plasmids (not the ligation mixtures) directly into the SURE cells. Notably, intermediary transformation was also tried using $5-\alpha$ bacteria, however these completely degraded the ITR sequences in all plasmids.

For transformation into XL-10 gold, the bacteria were thawed and $\beta$-mercapto-ethanol was dissolved in the mixture containing the bacteria. Following this, the ligation mixture was introduced into the bacteria and the tubes were gently stirred. The mixture was then incubated on ice for 30 minutes, following which they were heat pulsed for 30 seconds on a $42{ }^{\circ} \mathrm{C}$ water bath. The mixture was incubated on ice for 2 minutes and mixed with SOC medium. Subsequently, the bacterial mixture was shaken for 1 hour at $37{ }^{\circ} \mathrm{C}$. The bacteria were then spread on plates containing ampicillin antibiotics and incubated at 37 ${ }^{\circ} \mathrm{C}$ overnight. The next day, colonies were picked for plasmid purification. Plasmids were purified one day afterwards using the QIAprep Spin Miniprep Kit and purified additionally by being precipitated in ice-cold ethanol with sodium acetate, then washed with $70 \%$ ethanol and taken up in TE.

After these plasmids had been prepared, they were transformed into SURE cells. This step and all the subsequent ones were carried out identically as for the cAMPr pAAV expression plasmid.

\subsubsection{Preparation of viral particles}

In all cases, the viral vectors were prepared in the form of AAV6 viral particles in collaboration with my supervisor, Dr. Kügler, and the lab technicians, Sonja Heyroth and Monika Zebski. Three steps were undertaken to prepare the vectors. To begin, HEK cells were transfected with the viral particles, allowed to grow and lysed. Then, the first extraction step was conducted using gradient centrifugation. Finally, the second extraction step was conducted using heparin column affinity chromatography.

For production of viral particles using HEK cells, HEK293 cells were cultured inside cell factories at a density of $1 \times 10^{8}$ cells per cell factory. These cells were grown in DMEM (10\% FCS, 1\% PS) until reaching 50\% confluency, after which their medium was replaced with DMEM (2\% FCS). The cells were then transfected using the calcium-phosphate method, after which they were again incubated in DMEM (10\% FCS, 1\% PS). Around two days after transfection, the cells were lysed using the citric saline method. A sufficient degree of confluency was also required for deciding to lyse the cells ${ }^{84,113}$.

After the lysis of viral particles, iodixanol gradient ultracentrifugation was performed. In this procedure, iodixanol is used to create a set of density gradients, which are used to isolate components of a sample, 
such as the lysate containing AAV viruses acquired after virus production. More specifically, AAV viruses separate themselves into one of the bands, which can then be extracted using a sterile needle ${ }^{258}$.

The resulting solutions were then purified subsequently using fast-protein liquid chromatography on Heparin affinity columns ${ }^{84,113}$. Briefly, the solution is run through a column filled with heparin beads, which bind the AAV6 viral particles. After this, the rest of the lysate is washed off and the AAV6 viral particles are eluted with a solution that releases the AAV6 viral particles from the heparin column. The viral particles are then gathered as part of this eluate. Finally, the eluates are dialyzed extensively against PBS and the resulting virus samples are aliquoted and frozen at $-80{ }^{\circ} \mathrm{C}^{84,113,259}$.

For verifying the purity of the viral particles, Coommassie staining and a SDS-PAGE analysis was used. The number of viral genome copies was then determined using quantitative PCR (qPCR) and divided by the number 30 to determine the total number of transduction units (infectious viral particles).

Importantly, the number of transduction units of the viral sample was calibrated by comparing it to either previous preparation of the same virus or a virus expressing a similar protein (e.g. NLS-mCherry). This was done to ensure consistency of protein expression across different preparations of the same virus. Notably, in the case of the series of viruses used for studying wild-type synucleins, the transducing units were calibrated across all the viruses, as all these viruses expressed NLS-mCherry (Figure 8A).

For this calibration, two methods were used. For viruses expressing NLS-mCherry, neurons were transduced with viruses encoding NLS-mCherry at different amounts of transducing units during the first week of culturing, after which the level of mCherry fluorescence produced by the viruses were compared and the assumed number of transducing units corrected if the mCherry fluorescence did not match between the two viruses. For viruses not expressing NLS-mCherry, neurons were also transduced with viruses during the first week of culturing. After around 1 week, the neurons were lysed and the protein levels inside the lysates were compared using a Western blot. If protein amounts between two viral preparations were different, then the number of transducing units were corrected.

\subsubsection{List of used antibodies}

\begin{tabular}{|l|l|l|}
\hline Primary antibody and dilution & Secondary antibody and dilution & Used for figure: \\
\hline $\begin{array}{l}\text { Anti- } \alpha \text {-synuclein }(\text { Syn211) } \\
(\text { ThermoFisher, 32-8100, 1:1000) }\end{array}$ & $\begin{array}{l}\text { Anti-mouse HRP } \\
\text { (Dianova, 666, 1:3000) }\end{array}$ & Figure 21 \\
\hline $\begin{array}{l}\text { Anti- } \alpha \text {-synuclein } \\
(\text { BD Biosciences, BD610787, 1:1000) }\end{array}$ & $\begin{array}{l}\text { Anti-mouse HRP } \\
\text { (Dianova, 1:3000) }\end{array}$ & Figure 22 \\
\hline $\begin{array}{l}\text { Anti-neurofilament L (anti-NFL) } \\
(\text { Cell Signaling, 2837, 1:500) }\end{array}$ & $\begin{array}{l}\text { Anti-rabbit Cy2 } \\
\text { (Dianova, 1:250) }\end{array}$ & $\begin{array}{l}\text { Figure 25, Figure 31, } \\
\text { Figure 33 }\end{array}$ \\
\hline $\begin{array}{l}\text { Anti-MAP2 } \\
\text { Sigma, ab5622, 1:500) }\end{array}$ & $\begin{array}{l}\text { Anti-rabbit Cy2 } \\
\text { (Dianova, 1:250) }\end{array}$ & $\begin{array}{l}\text { Figure 25, Figure 31, } \\
\text { Figure 33 }\end{array}$ \\
\hline $\begin{array}{l}\text { Anti-synapsin } \\
\text { Synaptic Systems, 1:500) }\end{array}$ & $\begin{array}{l}\text { Anti-rabbit AlexaFluor 350 } \\
\text { (ThermoFisher, 1:250) }\end{array}$ & Supplementary Figure 1 \\
\hline
\end{tabular}

Table 2. Table of antibodies used in different experiments.

The primary and secondary antibodies have been indicated alongside the used dilution, as well as the figure corresponding to the experiment that they were used in. HRP = horse radish peroxidase, $\mathrm{Cy} 2=$ cyanine. 


\subsubsection{List of used macros, programs and calculation sheets}

\begin{tabular}{|l|l|l|}
\hline Name & Type & Used for: \\
\hline mCherry_segmentation & ImageJ macro & $\begin{array}{l}\text { Segmenting NLS-mCherry images for identifying cell } \\
\text { locations }\end{array}$ \\
\hline stack_deflicker & ImageJ macro & $\begin{array}{l}\text { Correcting the movement artefact and the exposure } \\
\text { time variation of GCaMP6 calcium activity videos }\end{array}$ \\
\hline FluoroSNNAP 260 & Stand-alone program & $\begin{array}{l}\text { Detection of individual cell depolarizations from a } \\
\text { calcium activity video }\end{array}$ \\
\hline GraphPad Prism & Stand-alone program & Visualization of data, statistics \\
\hline Affinity Designer & Stand-alone program & Illustrations for figures \\
\hline burst_identifier & Excel spreadsheet & $\begin{array}{l}\text { Determining the number of network bursts and the } \\
\text { percentage of neurons involved in network bursts }\end{array}$ \\
\hline presynapse_quantifier & ImageJ macro & $\begin{array}{l}\text { Determining the number of active presynapses from a } \\
\text { video of presynaptic calcium activity. }\end{array}$ \\
\hline neurite_quantifier & ImageJ macro & $\begin{array}{l}\text { Determining the amount of neurites from an image of } \\
\text { ICC-stained axons or dendrites }\end{array}$ \\
\hline akar_divider & ImageJ macro & $\begin{array}{l}\text { Generating a FRET ratio image from the } \\
\text { AKAR4-cAMPs CFP and YFP signals }\end{array}$ \\
\hline scanner & ImageJ macro & $\begin{array}{l}\text { Analyzing the FRET ratio image to determine the } \\
\text { average FRET ratio per neuron (AKAR4-cAMPs) } \\
\text { Analyzing the cAMPr image to determine the average } \\
\text { level of neuronal cAMPr fluorescence (cAMPr) }\end{array}$ \\
\hline
\end{tabular}

Table 3. List of macros and programs used over the course of the doctoral thesis.

The various programs and ImageJ macros used in this study have been indicated. NLS-mCherry = nuclear localization signal tagged mCherry, CFP = cyan fluorescent protein, YFP = yellow fluorescent protein, FRET = Förster resonance energy transfer.

\subsubsection{List of substances}

\begin{tabular}{|l|l|l|}
\hline Substance & Target and function & Used for figure: \\
\hline Rolipram (Abcam, $1 \mu \mathrm{M})$ & Phosphodiesterase inhibitor & Figure 27 \\
\hline Rolipram (Abcam, $5 \mu \mathrm{M})$ & Phosphodiesterase inhibitor & Figure 6, Figure 7 \\
\hline Forskolin (Abcam, $5 \mu \mathrm{M})$ & Adenylate cyclase activator & Figure 6, Figure 7 \\
\hline Linderane (Cayman, $20 \mu \mathrm{M})$ & Phosphodiesterase activator & Figure 6, Figure 7 \\
\hline SQ-22536 (Cayman, $20 \mu \mathrm{M})$ & Adenylate cyclase inhibitor & Figure 6, Figure 7 \\
\hline ZD-7288 (Sigma, $10 \mu \mathrm{M})$ & HCN inhibitor & Figure 26 \\
\hline BDNF (Cell Signaling, 2 nM) & TrkB agonist & Figure 26 \\
\hline
\end{tabular}

Table 4. List of substances used in the various figures.

The substances used over the course of the study have been indicated, alongside their function and the figure that they were used in. $\mathrm{HCN}=$ hyperpolarization-activate cyclic nucleotide gated cation channel, TrkB = tyrosine receptor kinase $\mathrm{B}, \mathrm{BDNF}=$ brain-derived neurotrophic factor.

Substances used in the experiments were ordered from the manufacturer, following which they were dissolved in DMSO or water, aliquoted and stored at $-20{ }^{\circ} \mathrm{C}$ (Table 4). For application of the substances alongside imaging, two methods were used. When using the inverted microscope, neuron plates were treated with the substance, which was pipetted into the medium in such a volume that it would be diluted 
to the final concentration. After this, the cell plate was incubated inside the incubator for 30 minutes and then recorded.

When using the immersion microscope, the substances were diluted inside the perfusion solution. These solutions were then introduced onto the neurons over the course of normal perfusion - the normal perfusion solution was switched to the solution containing the substance.

\subsection{Analytical methods}

\subsubsection{Recording of neuron network bursts}

\subsubsection{Transduction of neurons for analyzing network bursts (wild-type synucleins, tau protein)}

For the recording of neuron network bursts, neurons were transduced on DIV2 with $3 \times 10^{7}$ AAV virus particles encoding $\mathrm{Bcl}-\mathrm{xL}$ as well as $3 \times 10^{7} \mathrm{AAV}$ virus particles encoding GCaMP6. After this, neurons were transduced on DIV4 with $2 \times 10^{8} \mathrm{AAV}$ virus particles encoding either a synuclein or tau protein cDNA (Figure 8A). The neurons were then incubated and fluorescence of these neurons was recorded on DIV10, DIV16, DIV19, DIV22, DIV25, DIV28 and DIV31.

\subsubsection{Transduction of neurons for analyzing network bursts (mutant synucleins)}

For the mutant synucleins, neurons were otherwise transduced identically as for wild-type synucleins and tau protein. However, on DIV4, a different set of AAV viruses was used (Figure 8B). Furthermore, on DIV4 the viruses were also transduced with 3x107 AAV virus particles encoding NLS-mCherry by itself. After this, the neurons were incubated and the fluorescence of these neurons was recorded on DIV16, DIV25, DIV28 and DIV31.

\subsubsection{Neuron recording setup}

For neuron recording, an Observer Z1 (Zeiss) microscope was used, which is an inverted microscope. This microscope was equipped with a plate incubator, which kept the cell plates in an environment consisting of $37{ }^{\circ} \mathrm{C}$ and $5 \% \mathrm{CO}_{2}$ while they were being recorded. For this, the atmosphere was regulated by a Temp Module $\mathrm{S}$ (Zeiss) and a $\mathrm{CO}_{2}$ Module $\mathrm{S}$ (Zeiss), whereas the plate was contained inside a heating insert M24 2000 EC (Pecon) and an Incubator PM 2000RBT (Pecon). The heating insert and incubator were furthermore placed on a Zeiss microscope stage, which allowed for the manual movement of a culture plate. In order to supply the $\mathrm{CO} 2$, a properly secured carbon dioxide canister equipped with a pressure release regulator was installed near the microscope. The microscope was also powered using a Power Supply 322 (Zeiss).

To supply the microscope with light, a Zeiss HXP 120V device was used, which was normally operated at the default setting (50\% light strength). The microscope was equipped with a variety of filter cubes, of which two were required for analyzing the network bursts of neurons - a 43HE DsRed filter (Zeiss) 
for NLS-mCherry detection and a GFP filter (Semrock) for GCaMP6 detection. For magnification, a Fluar 5x objective (Zeiss) was used with a 0.25 aperture.

\subsubsection{Recording NLS-mCherry fluorescence and the calcium activity of neurons}

In order to acquire images for the detection of neuron network bursts, two sets of fluorescent images were required. To begin, it was necessary to take a $2 \times 2$ binned static image of the NLS-mCherry fluorescence, which permitted the detection of neuron locations. Subsequently, a 2x2 binned video of the activity of the GCaMP6 calcium sensor was recorded. Importantly, for recording the calcium sensor activity, the exact same area of neurons was recorded, thus making it possible that the neurons identified using NLS-mCherry fluorescence would be the exact same neurons recorded in the GCaMP6 calcium sensor activity video (Figure 9).

A

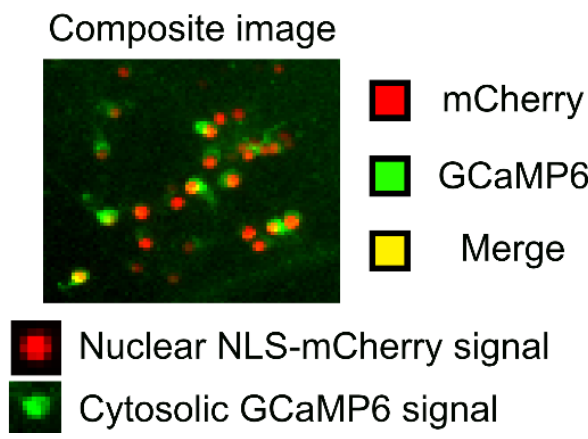

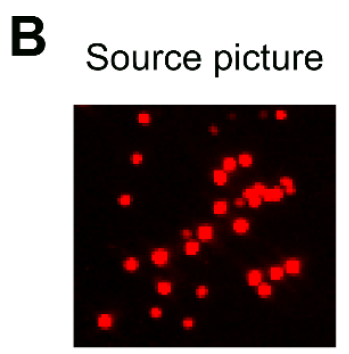

Nucleus expressing NLS-mCherry

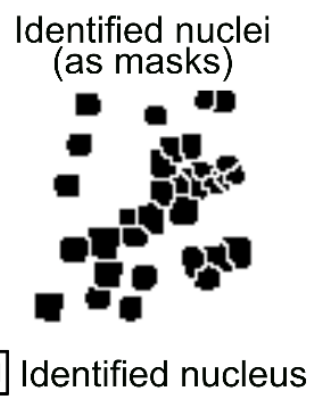

Figure 9. Segmentation of cell nuclei using the "mCherry_segmentation" macro.

(A) Composite image of NLS-mCherry expressing nuclei (red) alongside GCaMP6 expressing cytosols (green). Both images were acquired from neurons expressing $\gamma$-synuclein from DIV4 to DIV28. The green component of the image was acquired from a video of GCaMP6-expressing neurons during calcium activity. The red component was acquired by taking a separate image of mCherry-expressing nuclei.

(B) Results of an automated identification procedure for nuclei. A source picture of mCherry-expressing nuclei (red fluorescence, left panel) and a post-processing image of identified nuclei (black shapes, right panel) are indicated.

Before recording the neurons, the Greiner culture plate was extracted from the cell culture incubator and placed inside the microscope plate incubator for the duration of the measurement. During the measurement a set of standard practices was used. This included measuring the neurons in the plate incubator for no longer than 30 minutes and avoiding unnecessary illumination of the neurons using the microscope. These steps were taken in order to ensure minimal aggravation of the neurons during imaging, thus enabling the neurons to survive for multiple measurements.

For recording the NLS-mCherry fluorescence of the neurons, an area of the neuron culture was selected which was about $50 \%$ of the distance between the left edge of the culture well and the center of the well, which usually contained 2000-3000 neurons. After this, a stack of images from taken of these neurons, which consisted of several images. Firstly, the stack contained NLS-mCherry images taken at several exposure times ranging from $50 \mathrm{~ms}$ exposure to $1 \mathrm{~s}$ exposure. After this, one static image was recorded of the GCaMP6 signal using $500 \mathrm{~ms}$ exposure and one image was also recorded using brightfield illumination. 
After the NLS-mCherry image stack was taken, the exact same location was recorded to detect the calcium sensor signal. For this, every one second an image of the GCaMP6 signal was taken using a 500 $\mathrm{ms}$ exposure time (1 Hz frame rate) for a total of 60 images. Importantly, this frame rate was selected as neuron network bursting involves a long-lasting calcium influx into the neurons, which could be detected easily even when the temporal resolution was very low.

Once both the static NLS-mCherry image and the calcium sensor video had been taken, the next well was recorded using the same protocol. Only one image per culture plate well was taken as in most cases, taking more pictures was unnecessary due to the uniform character of network bursting across the entire culture. Furthermore, taking more pictures would not always have been possible because of the 30 minute time limit for imaging one neuron culture plate.

\subsubsection{Segmentation of the image using the NLS-mCherry image stack}

After the images were acquired, they were analyzed using an automated procedure designed in ImageJ (Table 3, "mCherry_segmentation") ${ }^{261}$. In the first step, the stack of static images was processed, with the NLS-mCherry frames of this image being used to identify the locations of cells the image. Notably, these frames consisted of nuclei filled with NLS-mCherry, which were observable on the image as strongly fluorescent spherical structures (Figure 9). These spherical structures were optimal for identifying cell locations, as automated computer analysis procedures identify circular structures more easily than structures with a complicated shape (such as neurons). Notably, localization of the nuclei also enable the localization of cytosols, as in neurons, the cytosol surrounds the nucleus.

For segmentation of the image, a custom-made ImageJ macro was used. This macro was designed to identify structures of interest (i.e. the cells) from the image based on three principles. Firstly, it was necessary to only identify structures, which were substantially brighter in the image than the background pixels. Secondly, it was necessary to separate clumps of structures, such as clustered neurons. Finally, it was necessary for the structure to be sufficiently large (at least 30 pixels), which would prevent bright pieces of cellular debris from being identified as neuronal nuclei.

In order to accomplish the first goal, the image was first pre-processed by several background subtraction steps (including ImageJ utility "Subtract Background"), which significantly increased the contrast between the neuronal nuclei and the background ${ }^{261}$. Following this, Phansalkar local thresholding was performed, where the program would identify neuronal nuclei based on their relatively higher brightness compared to background pixels ${ }^{262}$.

After this, the image was segmented a second time for the identification of dim structures. This was necessary because the Phansalkar thresholding method is not capable of identifying dim structures when they are located immediately next to a bright structure. In order to analyze the image a second time, all of the neuronal nuclei identified during the first round of Phansalkar thresholding were subtracted from 
the original image. After this was performed, Phansalkar thresholding was applied on the image again, thus detecting the dimmer neurons.

After structure identification, clusters of neurons were separated. For this, a maxima-based watershed method was used. This method made use of the fact that each fluorescence signal of a NLS-mCherry marked nucleus contained only one local maximum (spot of brightest fluorescence). Therefore, clustered neurons could be separated using these local maxima, as each neuron only had one. In order to achieve this, utilities included with the ImageJ platform were used, including a maxima-locating program and a maxima-based watershed ${ }^{261,263}$.

After separating the neuronal clusters into individual neurons, it was necessary to filter these neurons for size. In this stage of analysis, the image had been processed into binarized masks, with identified structures taking the form of black structures on a fully white background (Figure 9). These structures were then filtered for size using the "Analyze Particles" utility in ImageJ. Structures composed of 120 pixels or more (30 pixels or more was also used in some cases) were identified as neurons, whereas the rest were excluded as debris.

Finally, the amount of identified structures was counted to determine the number of neurons in the image. Finally, the masks of the identified neurons were slightly enlarged, having the impact of enlarging the contours of the identified neurons for the next stage of analysis. This was done to ensure that the neuronal cytosol was included inside the mask (contour).

\subsubsection{Segmentation settings}

Importantly, the macro used for segmentation enabled the use of multiple settings. Firstly, the NLSmCherry image used to segment the image could be altered. Namely, some NLS-mCherry pictures were taken with a higher exposure time, resulting in the image having a greater brightness. Others were taken with a lower exposure time, resulting in the image having a lower brightness. If higher exposure time pictures were used for segmentation, the analysis would be more sensitive but the false detection rate would be increased. If lower exposure time pictures were used for segmentation, the opposite would be the case.

Secondly, the size filter of the analysis algorithm could also be altered. Namely, it could be selected whether the analysis would require structures to be 30 pixels in size or larger to be detected as neurons or as much as 120 pixels in size or larger. If the former criterion was used, the analysis would be more sensitive but the false detection rate would be increased. If the latter criterion was used, the opposite would be the case.

In this study, NLS-mCherry images with an exposure time of $1000 \mathrm{~ms}$ were segmented when the image was acquired on DIV10. Furthermore, when processing images from this timepoint, structures 30 pixels in size or larger were detected as neurons. These settings were selected for this timepoint, as at this 
timepoint, the increased false detection rate was not particularly important - less debris was apparent in the neurons at this timepoint than the subsequent timepoints due to the culture not being aged. In contrast, the increased sensitivity was considered advantageous, as it would increase the chance of detecting the neurons, which possessed only a medium intensity of fluorescence on DIV10.

Meanwhile, NLS-mCherry images with an exposure time of $500 \mathrm{~ms}$ were segmented when the image was acquired on DIV16 or later. Furthermore, when processing images from this timepoint, structures that were 120 pixels in size or larger were detected as neurons. These more strict settings were required for this timepoint, as at this timepoint, more fluorescent debris was present in the culture medium, which were falsely detected as neurons under more lenient settings.

\subsubsection{Pre-processing of the GCaMP6 calcium activity recording}

Notably, the GCaMP6 calcium activity recording would occasionally contain artefacts caused by either faults of the imaging equipment or by experimental error. The most common artefact was a "flickering" phenomenon, where some frames of the calcium recording were brighter than other frames, which was caused by small fluctuations of the exposure time. The second most common artefact was a movement artefact, where the neurons in the video moved slightly compared to their initial position. This was likely caused by accidentally pushing the microscope or the table that it was placed on.

In order to correct this artefact, a separate ImageJ macro was created (Table 3, "stack_deflicker"). This macro performed two tasks. Firstly, the flickering artefact was corrected using a "Stack Deflicker" ImageJ utility (designed by Jesper Pedersen), which also corrected the image for bleaching ${ }^{261}$. Secondly, the movement artefact was corrected using the "StackReg" ImageJ utility (designed by Philippe Thevenaz) ${ }^{261}$.

\subsubsection{Analysis of the calcium activity of the neurons to determine individual neuron depolarizations}

After pre-processing the calcium activity recording, the next step was to determine the depolarizations of individual neurons based on the calcium activity recording, for which the program FluoroSNNAP was used ${ }^{260}$. Notably, both the identified neuron locations (in the form of binarized masks) and the preprocessed calcium activity recording were used for this analysis step.

As the first step, the neuron locations were converted to the format used by the FluoroSNNAP program. For this, the binarized masks were processed using the "Threshold" tool of the program's "Segmentation GUI" utility, which allowed the program to recognize the locations and to save them as a file compatible with Matlab (the coding language of FluoroSNNAP).

Following this, the measurement settings were modified as follows. Firstly, in the setting screen "Analysis Modules", only the basic analysis was selected, for which all verification boxes in the screen were unchecked. Secondly, in the setting screen "Acquisition Frame Rate", the frame rate was set to 1 
frame per second. Thirdly, in the settings screen "Baseline Fluorescence", 3 seconds was used as the time window, whereas $10 \%$ was used as the "Percentile for baseline fluorescence".

After this, the images were analyzed by the program as described in Patel et al. ${ }^{260}$. Briefly, the image was processed in several steps. Firstly, the program determined the average fluorescence of the neurons over the course of the calcium imaging video, after which the fluorescence was normalized by calculating the factor $\left(F-F_{0}\right) / F_{0}$, where $F$ is the fluorescence and $F_{0}$ is the lowest decile of the fluorescence values measured over the previous 3 seconds. This normalized fluorescence was then used to detect peaks using a spike detection algorithm custom-prepared by Patel et al., which involved comparing the spikes of the normalized fluorescence to a database of possible calcium spike shapes ${ }^{260}$. Based on this, the program produced a raster file, where depolarization events for each neuron over 60 seconds were recorded. These results could be visualized in a variety of ways, for instance as a raster plot (Figure 10).

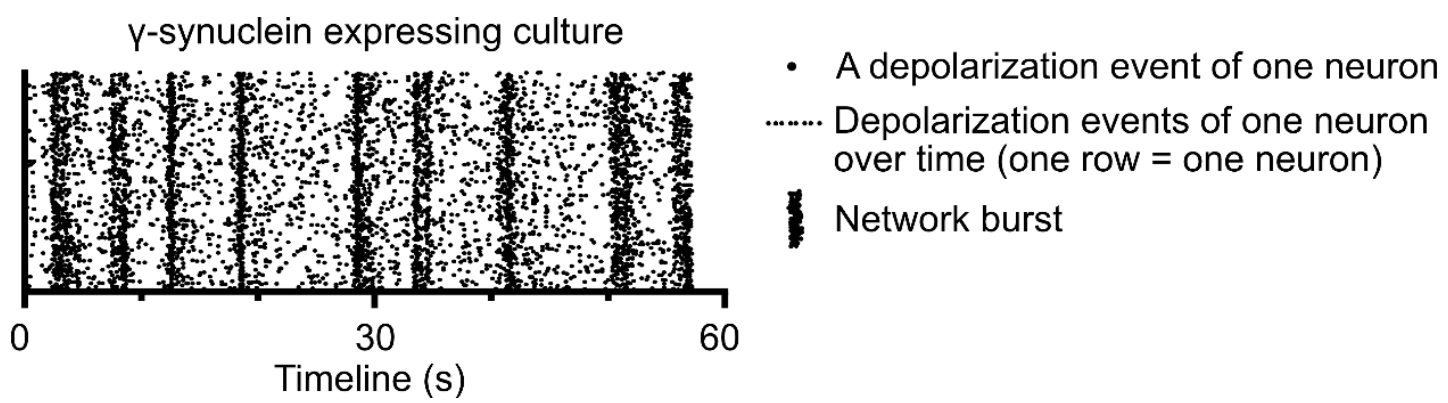

Figure 10. Example raster plot produced by the FluoroSNNAP program.

An example raster plot demonstrating non-stimulated network bursting for cultures overexpressing $\gamma$-synuclein from DIV4 to DIV28. Dots aligned in a row = depolarization events by an individual neuron. The data for this raster plot was generated by FluoroSNNAP, whereas the raster plot design was generated using GraphPad Prism.

\subsubsection{Analysis of individual neuron depolarizations to determine network bursts}

After the FluoroSNNAP program had been used to determine individual neuron depolarizations, the number of network bursts was determined alongside the amount of neurons contributing to each burst, which was done using a custom-made Excel spreadsheet ("burst_identifier", Table 3). As mentioned, network bursts are a type of neuron culture event, where a large segment of the entire culture becomes depolarized over the course of a $\sim 5$ seconds. Thus, the basic concept for identifying network bursts was to determine the rate of network activation over the recording in 5 second time windows. If this percentage of activation was significantly higher than the percentage of activation during regular activity, the event was identified as a network burst. Notably, time windows were used as neurons involved in the same network burst underwent depolarization during different seconds - some neurons would depolarize a second earlier and some a second later than others.

To detect network bursts, the FluoroSNNAP analysis data was exported in the form of a spreadsheet containing the exact time at which an individual neuron depolarization event was detected (in seconds from beginning of recording). This data was then entered into the "burst_identifier" Excel spreadsheet. In this spreadsheet, the number of neurons (as \% of total neurons) undergoing a depolarization were 
determined for each second of the recording. On the basis of these values, the number of neurons (as \% of total neurons) was determined that underwent a depolarization inside a 5-second time window.

These 5-second time windows were then used to identify network bursts. A network burst was registered when the amount of depolarizing neurons (as \% of total neurons) inside a time window was $10 \%$ higher than the amount of depolarizing neurons (as \% of total neurons) during a time window consisting of background activity. For instance, if $43 \%$ of the neurons depolarized during one time window and $12 \%$ during a time window consisting of background activity, a burst was considered to have taken place in the first time window.

After the burst was identified, the percentage of total neurons involved in this activity was also determined. For this, the amount of depolarizing neurons during the background activity was subtracted from the amount of depolarizing neurons during the network bursts. For instance, in the example inside the above paragraph, the percentage of total neurons involved in that network burst would have been determined to be $31 \%$ ( $12 \%$ subtracted from $43 \%$ ).

After the analysis had been performed, the number of network bursts was stored in GraphPad Prism software. The percentage of total neurons involved in each network burst was stored as well.

\subsubsection{Data visualization}

For data visualization, three different types of software were used - GraphPad Prism, Affinity Designer and Microsoft Word.

GraphPad Prism was used for several purposes. To begin, it was used as the main software for storing the numerical values from various experiments and furthermore, to compile the results of different repeats of the same experiment. GraphPad Prism was also used to generate a variety of different graphs. Basic graphs were generated as "Column" type graphs (e.g. Figure 3), whereas the majority of graphs were generated as "Grouped" type graphs (e.g. Figure 18). Beyond the creation of graphs, GraphPad Prism was also used to create scatter plots (e.g. the plot in Figure 18).

In all cases, data was displayed as a column graph with an error bar, with the height of the column graph indicating the mean of the sample, whereas the error bar depicted the standard deviation of the sample (e.g. Figure 18). In some cases, the means were also indicated further as values organized into a table, as in Figure 21.

Meanwhile, additional illustrations were created using vector graphics software Affinity Designer. In some cases, the illustrations were created from geometric shapes (e.g. Figure 8). In other cases, it was used to create illustrations from recordings of neurons, which were exported from the original images using ZEN software (Zeiss) or ImageJ. Finally, table figures were created with the help of Microsoft Word. 


\subsubsection{Statistical analysis of data}

\subsubsection{Unpaired two-tailed t-test with Welch's correction}

To compare whether the mean of two samples is different, our study used an unpaired two-tailed t-test with Welch's correction. For this, our study used GraphPad Prism software. An example of a figure using this type of statistical analysis is Figure 18.

In general, the t-test is a type of analysis involving two hypotheses, with one proposing that the two compared means are not different (that the means are equal), whereas the other one proposes that the two compared means are different (the means are not equal). In this test, a t-statistic is calculated from the means, standard deviations and sample sizes of the datasets. Following this, the p-value is calculated, which indicates the chance that two randomly selected sample datasets would have means as far apart as the two means in the comparison ${ }^{264}$. A low p-value thus indicates a greater likelihood that the two means being compared are indeed different from each other.

Meanwhile, our study used an unpaired t-test, as our compared samples were not matched - the neuron populations that were being compared were not the exact same population under a different treatment, but two independent neuron populations grown separately. Furthermore, Welch's correction was used, as this is the type of test that is used when it is not known if the variance (standard deviation) of the compared samples is different or identical. Finally, a two-tailed t-test was used, as this type of test is used when it is not known which of the compared samples is larger - a one-tailed t-test can only be used when it is known one of the two sample means is larger ${ }^{264}$.

In order to determine if the two calculated means were significantly different, the p-values were compared to different $\alpha$, which is a value indicating the cutoff point, below which $p$-values indicate a significant difference. For this study, the $\alpha$ was selected to be 0.05 , as per convention. Additionally, the size of the p-values were indicated on the graphs using asterisks. The meaning of the asterisks are as follows $-*$ indicates $0.01<\mathrm{p}<0.05$, ** indicates $0.001<\mathrm{p}<0.01$, *** indicates $0.0001<\mathrm{p}<0.001$, $* * * *$ indicates $\mathrm{p}<0.0001{ }^{264}$.

\subsubsection{Post-hoc statistical power test}

In order to determine the post-hoc statistical power of the sample comparisons, GPower 3.1. software was used ${ }^{265}$. In terms of what it describes, statistical power is a numerical value that indicates the chance of identifying a significant difference using the given sample size and effect size, assuming that such a difference actually exists. A higher statistical power thus indicates a higher likelihood of finding actual significant differences between two samples ${ }^{264}$.

For determining post-hoc statistical power, a two-tailed test for two independent means was used, which calculated the statistical power as a function of the size of the samples, the means of the samples and 
the standard deviations of the samples. In our study, the statistical power was determined for all statistically significant comparisons and included in the figure legend.

\subsubsection{One-way ANOVA}

To determine whether more than two population means were equal, a one-way analysis of variance (ANOVA) with Tukey's multiple comparisons test was used. For this, GraphPad Prism software was used. In terms of what it describes, the basic type of comparison performed by the ANOVA test is similar to the basic type of comparison by the t-test, except that the means of more than two samples are compared. The calculation process is also different, with the p-value determined by calculating a socalled F ratio, not a t-statistic, as in the case of the t-test ${ }^{264}$.

\subsubsection{Quantification of protein amounts using a Western blot analysis}

\subsubsection{Lysate acquisition}

For gathering lysates and culture mediums, a solution was prepared containing Tris (1 M) sodium dodecyl sulphate (SDS, 0.5\%), dithiothreitol (DTT, $100 \mathrm{mM}$ ) and protease inhibitor (PI, $1 \mathrm{x}$ ). After this, culture plates containing neurons were taken from the incubator, their medium was gathered into Eppendorf tubes and the culture wells were washed. Following this, $80 \mu 1$ of the lysis buffer was pipetted into the culture well. The lysis buffer was then applied on the neurons and the neurons were scraped from the coating with a pipette tip that had been cut to have a blunt wide end ${ }^{84}$.

Following this, the lysate was pipetted into an Eppendorf tube, quickly centrifuged and then sonicated with an ultrasonic homogenizer (UW 270 Bandelin Electronics) for 10 seconds. Following this, the neurons were centrifuged for 30 minutes at $13000 \mathrm{rpm}$ and $4{ }^{\circ} \mathrm{C}$. After the centrifugation was done, the supernatant was extracted, aliquoted and stored at $-20{ }^{\circ} \mathrm{C}{ }^{84}$. The previously gathered culture mediums were also aliquoted and stored at $-20^{\circ} \mathrm{C}$.

\subsubsection{Bicinchoninic acid (BCA) assay}

For determining the concentration of protein inside the lysates, a BCA assay was used. For this assay, the lysates were thawed and diluted, whereas to provide a point of comparison, various dilutions of a bovine serum albumin (BSA) standard were also prepared. Then, $10 \mu 1$ of the dilutions were loaded onto the plate, after which the dilutions were treated with $190 \mu \mathrm{l}$ of a mixture consisting of reagents $\mathrm{A}$ and reagents B of the Pierce BCA Protein Assay Kit (ThermoFisher). The 96-well plate was then incubated inside a $37^{\circ} \mathrm{C}$ incubator for 30 minutes.

After incubation, the 96-well plate was extracted and transferred into a Spark 10M plate reader (Tecan), which measured the absorbance of each well at a $562 \mathrm{~nm}$ wavelength. The measured absorbances were then transferred into an Excel table, where the known masses of the BSA standard and their measured absorbances were used to create the calibration curve. This calibration curve was then in turn used to 
determine the amount of protein in the sample wells, which was used to determine the concentration of the protein lysate.

\subsubsection{Preparing acrylamide gels}

Before conducting the Western blot, acrylamide gels were prepared. For this, an assembly consisting of a spacer glass and a short glass fixed using a casting frame was prepared. Following this, a mixture was prepared containing $125 \mathrm{mM}$ Tris, $0,1 \%$ SDS, $12-20 \%$ acrylamide, as well as acrylamide polymerization catalysts APS and TEMED. Before hardening of this mixture, it was transferred to the assembly so it could be hardened into a resolving gel. Before allowing the gel to harden, the top edge of the resolving gel was levelled by addition of $100 \%$ ethanol or isopropanol.

After the gel had fully hardened, the ethanol was poured off and the upper side of the gel was washed using water. After this, a mixture was prepared consisting of $375 \mathrm{mM}$ Tris, $0.1 \%$ SDS and $4 \%$ acrylamide, as well as the acrylamide polymerization catalysts APS and TEMED. The mixture was then pipetted on top of the resolving gel until reaching the edge of the two spacer and short glasses. After this, a comb was placed inside the gel, which was done to form slots for protein samples in the second layer of the gel. Subsequently, the second layer of the acrylamide gel was allowed to harden into the stacking gel. After this was complete, the acrylamide gel was wrapped inside wet tissue paper and stored in a bag at $4{ }^{\circ} \mathrm{C}$.

\subsubsection{Western blot procedure}

For performing the Western blot, lysates were extracted from the freezer and thawed, after which a precalculated amount of lysate was mixed with 6x SDS loading buffer (consisting of Tris, glycerol, SDS and bromophenol blue) and distilled water. Similar mixtures were also prepared from a protein standard (e.g. an $\alpha$-synuclein or $\gamma$-synuclein protein standard). Then, the mixture was heated at $95{ }^{\circ} \mathrm{C}$ for 5 minutes in order to ensure the denaturation of the protein's secondary structure. During this time, a pre-prepared acrylamide gel was placed inside a Western blot chamber (Bio-Rad) filled with electrophoresis buffer (25 mM Tris, $190 \mathrm{mM}$ glycine, 0.1\% SDS), following which the slots of the gel were washed with the same buffer.

After the Western blot mixtures had been heated, they were allowed to cool briefly, following which they were transferred onto the gel alongside a PageRuler protein ladder (ThermoScientific). The gel was then attached to an electrophoresis device and electrophoresed at $70 \mathrm{~V}$ for 20 minutes, after which it was electrophoresed at $100 \mathrm{~V}$ for as long as was needed for full separation of proteins on the gel. This process was tracked using the protein ladder.

After the gel had been fully run, they were moved into a room with $4{ }^{\circ} \mathrm{C}$ temperature. In this room, protein transfer from the gel to the membrane was conducted, for which a polyvinylidene fluoride (PVDF) Immobilon transfer membrane (Merck Millipore) was used. In order to facilitate the transfer, an assembly was created consisting of a sponge, blotting paper, gel, membrane, blotting paper and 
sponge. This assembly was then secured and transferred into a Bio-Rad membrane transfer device in such a way that the gel was aligned towards the cathode side of the device, whereas the membrane was aligned towards the anode side of the device, thus enabling transfer from gel to membrane.

Subsequently, the transfer device was placed inside the Western blot chamber and filled with $1 \mathrm{x}$ transfer medium (20\% methanol, $25 \mathrm{mM}$ Tris, $192 \mathrm{mM}$ glycine, $\mathrm{pH}$ 8.3). Protein transfer was then conducted at $100 \mathrm{~V}$ for 1 hour. Importantly, in order to prevent heating of the transfer buffer, a container filled with ice was added into the Western blot chamber and the chamber was surrounded on the outer side with additional ice.

After the transfer was completed, the membrane was transferred into a PBS solution containing $4 \%$ paraformaldehyde (PFA) and 0,1\% glutaraldehyde (GA). This was done to fix the proteins, thus minimizing protein loss during the subsequent experimental stages. The fixing procedure was carried out for 30 minutes at room temperature. In order to prevent spread of toxic PFA and GA gases, this experiment was conducted under a fume hood.

After the membrane was fixed, it was washed three times with a TBS-T solution (20 mM Tris, $137 \mathrm{mM}$ $\mathrm{NaCl}, 0.1 \%$ Tween-20) for 10 minutes each. Subsequently, the membrane was transferred into a blocking solution, which took the form of either milk powder (5\%) or BSA (4\%) diluted in TBS-T. In this solution, the membrane was then blocked for 60 minutes at room temperature.

During the blocking of the membrane, a dilution was prepared of the primary antibody inside the blocking solution inside a $50 \mathrm{~mL}$ Falcon tube (experiment-specific details on Table 2). After the blocking was done, the membrane was then transferred into this Falcon tube and rolled on a SRT6 rolling device (Stuart) at $4{ }^{\circ} \mathrm{C}$ overnight.

On the next day, the membrane was extracted from the Falcon tube and washed inside TBS-T three times for 10 minutes each. During the washing procedure, a dilution was prepared of a horse radish peroxidase (HRP) conjugated secondary antibody, which was selected to bind the primary antibody (experiment-specific details on Table 2). This antibody was the diluted inside the blocking solution inside a $50 \mathrm{~mL}$ Falcon tube. After the membrane was washed, it was subsequently transferred into this Falcon tube and rolled on a rolling device at room temperature for 1 hour.

After the membrane had been incubated with the secondary antibody, it was washed again inside TBS-T three times for 10 minutes each. During the washing, two solutions were heated to room temperature ECL solution 1 (2.5 mM luminol, $0.4 \mathrm{mM}$ p-coumaric acid, $100 \mathrm{mM}$ Tris) and ECL solution 2 (0.018\% $\mathrm{H}_{2} \mathrm{O}_{2}, 100 \mathrm{mM}$ Tris). After the membrane had been washed, the two ECL solutions were mixed together $1: 1$, after which the surface of the membrane was covered with the mixture and incubated at room temperature for 1 minute. Subsequently, the membrane was placed between two pieces of plastic and transferred to a PhosphoImager device. This was done to measure the light response, which is produced by the secondary antibody conjugated HRP enzyme when it oxidizes luminol in the presence of 
hydrogen peroxide. The strength of this light intensity was thus proportional to the amount of secondary antibody, which was in turn proportional to the amount of primary antibody and through that, the amount of protein inside the membrane ${ }^{84}$.

Importantly, four technical replicates were conducted for each Western blot membrane. This involved four identically loaded Western blot gels, which were treated identically.

\subsubsection{Determining the mass of protein samples}

For determining the mass of various protein samples, the strength of their Western blot signals was measured using Image Lab software (Bio-Rad), after which a calibration curve was prepared using the strength of the Western blot signals and the known masses of $\alpha$-synuclein standards (Figure 11). More specifically, a linear curve was prepared, for which the GraphPad "Simple Linear Regression" analysis modality was applied. Finally, in order to predict the mass of protein samples from this linear curve, the "interpolate unknowns from standard curve" GraphPad Prism utility was used, with the input values being the sample band Western blot signal intensities.

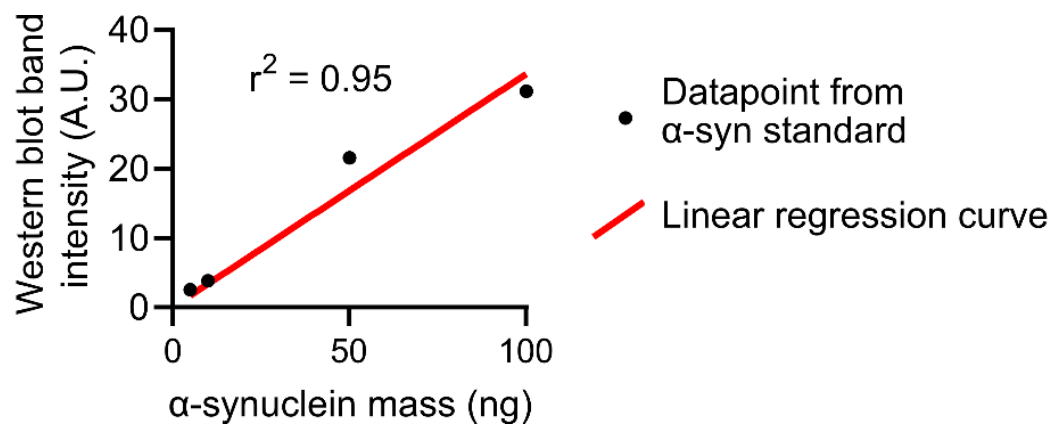

Figure 11. Example of a calibration curve used to determine $\alpha$-synuclein mass.

Visualization of a calibration curve used for $\alpha$-synuclein mass determination. Red line $=$ linear regression curve, points $=$ data points from an $\alpha$-synuclein standard. The $\mathrm{r}^{2}$ value has also been indicated.

\subsubsection{Determining the concentration of $\alpha$-synuclein inside and outside the neurons}

Notably, in Figure 21, the concentration of $\alpha$-synuclein was calculated both inside and outside the cultured neurons. For this, a formula was used for determining the molar concentration as a function of $\alpha$-synuclein mass, volume and molar mass (Figure 12).

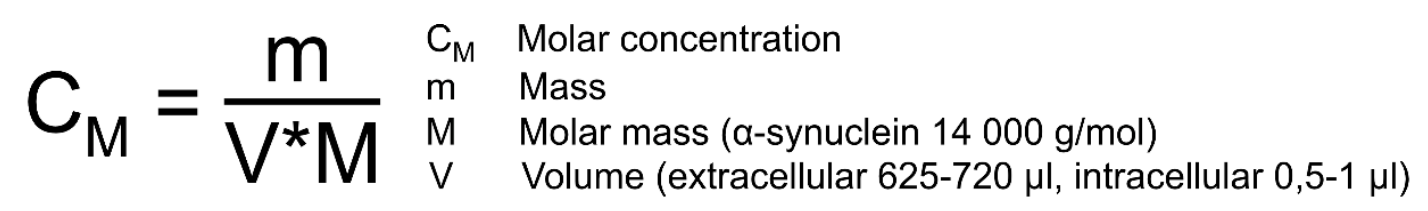

Figure 12. Formula used for calculating the intracellular and extracellular molar concentration of a-synuclein.

Image of the concentration calculation formula. $\mathrm{C}_{\mathrm{M}}=$ molar concentration $(\mathrm{M}), \mathrm{m}=$ mass $(\mathrm{g}), \mathrm{M}=$ molar mass $(\mathrm{g} / \mathrm{mol})$, volume $(\mathrm{L})$.

Of these parameters, the molar mass of $\alpha$-synuclein was acquired from the UniProt database. In order to determine the total mass of intracellular and extracellular $\alpha$-synuclein, a known fraction of the lysate or 
culture medium (e.g. $1 \%$ of the total) was loaded onto the Western blot gel alongside $\alpha$-synuclein protein standards of known mass. These were then used to determine the mass of the lysate/medium fraction, which was multiplied to determine the total mass of $\alpha$-synuclein (e.g. if $1 \%$ of the total was used, the mass was multiplied 100 times for the total mass).

For determining the volume of the cultured neurons used to prepare the lysate, the total number of neurons in the culture was determined using neuron counting, following which this number was multiplied by a previously published value of individual neuron volume ( 6 picoliters) ${ }^{266-268}$. Meanwhile, the volume of the extracellular medium was determined using pipette measurement.

\subsubsection{Studying the impact of extracellular $\alpha$-synuclein using cell culture supernatant exchange}

For conducting the medium exchange experiments, three populations of neurons were used. The first population of neurons (“donor neurons") was transduced with AAV viral particles inducing expression of synuclein, whereas the second population ("receptor neurons") was not. More specifically, the donor neurons were transduced on DIV2 with $3 \times 10^{7} \mathrm{AAV}$ transducing units for expression of $\mathrm{Bcl}-\mathrm{xL}$ and $3 \times 10^{7}$ AAV viral particles for expression of GCaMP6. Then on DIV4, the donor neurons were transduced with $2 \times 10^{8} \mathrm{AAV}$ transducing units for expression of $\alpha$-synuclein. Meanwhile, the receptor neurons was transduced only with viral particles encoding Bcl-xL or GCaMP6. The donor and receptor neurons were then cultured in parallel until the day of medium exchange.

For the experiment depicted on Figure 23, the medium exchange was conducted on DIV22. To conduct this medium exchange, the medium of the receptor neurons was removed, after which the entire medium was gathered from the donor neurons and transferred to the receptor neurons. After this, the neurons were recorded 1 day, 3 days and 9 days after the exchange in order to determine the frequency of network bursts.

For the experiment depicted on Figure 24, the medium exchange was conducted on DIV28. For this, the medium of the donor neurons was removed, after which the medium of the receptor neurons was gathered and transferred to the donor neurons. After this, the neurons were imaged 1 day and 3 days after the exchange in order to determine the frequency of network bursts.

\subsubsection{Quantification of pre-synapses using Sy-GCaMP}

\subsubsection{Transduction of neurons for analyzing network bursts}

For the quantification of presynapses, neurons were transduced on DIV2 with $3 \times 10^{7}$ AAV virus particles encoding Bcl-xL as well as $1 \times 10^{8} \mathrm{AAV}$ virus particles encoding Sy-GCaMP. After this, neurons were transduced on DIV4 with $2 \times 10^{8} \mathrm{AAV}$ virus particles encoding either a synuclein or tau protein (Figure 8). The neurons were then incubated and recorded on DIV16, DIV25, DIV28 and DIV31. 


\subsubsection{Recording synaptic calcium activity}

For recording the neurons, the same microscope and equipment was used as for the recording of network bursts, involving the inverted microscope Axio Observer Z1. However, instead of using a 5x objective, an LD-Plan 40x 0,6 aperture objective was used (Zeiss), which provided a larger magnification required for the detection of small synaptic structures.

Furthermore, to track the change of synaptic calcium activity over time, a $2 \times 2$ binned video was taken of the Sy-GCaMP3.5. signal using a GFP filter cube (Semrock). For this video, one image was taken every second for a total of 60 seconds. The exposure time for each image was $\sim 500 \mathrm{~ms}$, with the exposure time calibrated slightly at the beginning of each experiment to ensure a similar level of fluorescence as previous repeats of the same experiment. This was necessary, as the analysis algorithm was sensitive to the absolute amount of fluorescence.

\subsubsection{Analysis of the synaptic calcium activity video}

For image analysis, the "presynapse_quantifier" macro was used (Table 3). This macro used the previously acquired synaptic calcium activity video of a neuronal culture expressing Sy-GCaMP3.5. The basic design principle was to identify structures that underwent a calcium increase and then to count these structures as an indicator of the number of active presynapses (presynapses that underwent calcium activity).

More specifically, the macro first corrected the image for two types of artefacts that were also characteristic of the GCaMP6 recordings used to identify network bursts. Namely, the Sy-GCaMP3.5. videos exhibited a flickering artefact, where the exposure time of the image changed slightly, causing a one second increase of the fluorescence intensity of the image. In addition to this, the videos possessed movement artefacts caused by accidentally pushing the microscope or the desk that the microscope was stationed on. In order to correct the flickering artefact, the "Stack Deflicker" ImageJ utility was used, whereas to correct the movement artefact the "StackReg" ImageJ utility was used. Importantly, the "Stack Deflicker" method also functioned to correct the image for bleaching.

After the artefacts were corrected, ImageJ utilities were used to create a maximum projection and minimum projection image, which, respectively, were images depicting either the highest or lowest light intensity at each pixel location over the 60 second recording. After this, the minimum projection image was subtracted from the maximum projection image using the ImageJ "Image Calculator" utility ${ }^{261}$. The result was an image depicting the total change in calcium sensor fluorescence over 60 seconds at each pixel, with active synapses that underwent calcium influxes being strongly highlighted over debris and background structures.

After this, the level of background in the image was further reduced using the "Smooth", "Subtract Background" and "ROF denoise" utilities in ImageJ. Following this, two fold the average fluorescence of the image was subtracted to set the intensity values of all background pixels to 0 . The image was then 
binarized using the "Threshold" utility of ImageJ, yielding an image of black particles on a white background.

These particles were then filtered with size. More specifically, excessively small structures (structures smaller than 64 pixels) were excluded to prevent detection of artefacts. The number of the remaining structures was then counted. Subsequently, the number was divided by the size of the imaging area $\left(0.091 \mathrm{~mm}^{2}\right)$ in order to yield the number of active presynapses per $\mathrm{mm}^{2}$. This number was then recorded in GraphPad Prism for later statistical analysis.

\subsubsection{Determining the amount of axons and dendrites using immunocytochemistry}

In order to determine the amount of axons and dendrites, several steps were carried out. To begin, neurons were transduced and incubated until DIV16 or DIV28. After this, the neurons were fixed using PFA and stored at $4{ }^{\circ} \mathrm{C}$. Then, the neurons were extracted and stained with a primary antibody against either NFL (for axons) or MAP2 (for dendrites), after which they were visualized with a secondary antibody conjugated to a $\mathrm{Cy} 2$ fluorescent protein. Subsequently, the stained neurons were recorded using an Axio Imager Z2 microscope and finally, the amount of neurites was quantified using the custommade ImageJ macro "neurite_quantifier" (Table 3).

Notably, the axonal and dendritic marker were selected based on previously published studies, which indicated that NFL was abundant in axons, whereas MAP2 was abundant in dendrites ${ }^{253,269,270}$. In order to demonstrate that these markers indeed stained distinct structures, a culture of neurons were stained against both markers using ICC, revealing that distinct structures were indeed stained (Figure 13).

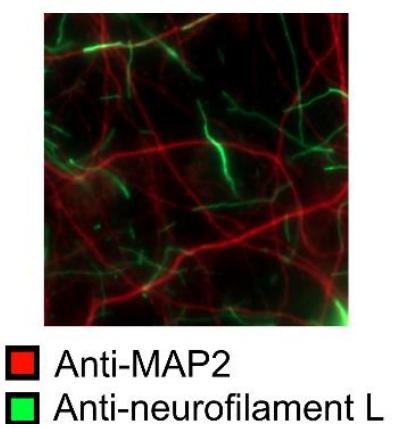

Figure 13. MAP2 and neurofilament $L$ are present in distinct neuritic structures, as determined by ICC staining.

Fluorescence image showing an immunocytochemical stain against MAP2 (red) and neurofilament L (green). The antibodies anti-neurofilament L (Cell Signaling) and anti-MAP2 (Sigma) were used.

\subsubsection{Transduction setup (wild-type synucleins, tau protein)}

For determining the amount of neurites, neurons were plated on culture plates containing coverslips. Then, neurons were transduced on DIV2 with $3 \times 10^{7} \mathrm{AAV}$ virus particles encoding Bcl-xL. After this, neurons were transduced on DIV4 with $2 \times 10^{8} \mathrm{AAV}$ virus particles encoding either a synuclein or tau protein (Figure 8A). The neurons were then incubated until DIV16 or DIV28, after which they were fixed, stained using immunocytochemistry and recorded. 


\subsubsection{Transduction setup ( $\beta$-synuclein P123H mutant)}

For determining the impact of the $\beta$-synuclein P123H mutant, a similar transduction setup was used. However, on DIV4, a different set of synuclein-encoding AAV viruses was used (Figure 8B). Furthermore, on DIV4 the viruses were also transduced with 3x107 AAV virus particles encoding NLSmCherry.

\subsubsection{Fixing plates using $4 \%$ PFA}

For fixing the plates, culture plates containing neurons were extracted from the culture incubator on DIV16 or DIV28. Subsequently, the culture medium of the neurons was removed and the neurons were washed twice with PBS. After this, the neurons were treated at room temperature for 15 minutes with a 4\% paraformaldehyde (PFA) dilution in PBS.

Once this treatment was done, the neurons were washed twice with PBS for five minutes each. Following this, PBS containing $0.02 \%$ sodium azide was pipetted on neurons, after which the culture plates were wrapped in parafilm and stored at $4{ }^{\circ} \mathrm{C}$ until immunocytochemistry.

\subsubsection{Staining neurons using immunocytochemistry}

For immunocytochemistry, the plates were brought to room temperature. Following this, the sodium azide containing PBS was removed and the neurons were treated for 5 minutes at room temperature with T-PBS (PBS containing 0.1\% Triton X100). During this step, a blocking solution was created consisting of $5 \%$ normal goat serum (NGS) and 1\% bovine serum albumin (BSA) inside T-PBS. After the neurons had been treated with T-PBS, the neurons were treated for 1 hour at room temperature with the blocking solution. In order to ensure that the neurons were blocked evenly, the neurons were shaken using a PSU-20i plate shaker (Grant bio) for the duration of this treatment.

During the blocking, an antibody dissolution mixture was created, for which 5\% NGS was diluted in T-PBS. After creating this mixture, dilutions were created of the primary and secondary antibody. For imaging axons, a 1:500 dilution of anti-NFL primary antibody was created, whereas for imaging dendrites, a 1:500 dilution of anti-MAP2 primary antibody was used. For both primary antibodies, a Cy2 conjugated anti-rabbit secondary antibody was utilized, which was diluted 1:250 (Table 2).

After the neurons had been blocked, the blocking solution was removed and the neurons were treated with the dilution of the primary antibody overnight at $4{ }^{\circ} \mathrm{C}$. During the incubation, the plate was shaken using an ST5 plate shaker (neoLab). For the duration of this incubation, the secondary antibody dilution was stored at $4{ }^{\circ} \mathrm{C}$.

The subsequent day the primary antibody dilution was removed and the neurons were washed 3 times with T-PBS for 5 minutes each. After this was complete, the neurons were treated with the secondary antibody dilution at room temperature for 1 hour, during which the plate was shaken using the PSU-20i 
plate shaker. Subsequently, the secondary antibody dilution was removed and the neurons were again washed with T-PBS for 5 minutes each.

Following this, the coverslip containing neurons was extracted using sterile pincers, washed with water, dried and mounted on microscope slides using Mowiol (Roth). Following this, the coverslips were ready for imaging.

\subsubsection{Recording ICC-stained neurites using fluorescence microscopy}

In order to record the neurites, the general intention was to acquire an image of both the neurites as well as well as image of the nuclei expressing NLS-mCherry. The second image was acquired to identify the location of cytosols (located peripherally to the nuclei), which were subtracted from the image during the analysis.

In order to acquire the images, a set of devices was used consisting of the Axio Imager Z2 microscope (Zeiss), the HXP 120V light source (Zeiss) and the microscope power supply 232 (Zeiss). The microscope furthermore utilized a Plan-Apochromat 40x 0.95 aperture objective (Zeiss) to achieve a sufficient magnification to detect neurites. For identifying the NLS-mCherry signal, a 45 Texas Red objective (Zeiss) was used, whereas for identifying the Cy2 signal, a "GFPxtra" filter was used with the following settings - emission range 473-491 nm, beam splitter $495 \mathrm{~nm}$, excitation range 506-534 nm. In both cases, the light source was operated at $48 \%$ intensity.

Thus, in order to record the neurites, microscope slides with the mounted coverslips were transferred to the Imager Z2 microscope. Following this, a stack of unbinned static images was acquired, consisting of several images of the NLS-mCherry signal with exposure times ranging from 50 to $1000 \mathrm{~ms}$ as well as several images of the Cy2 signal with exposure times ranging from 250 to $4000 \mathrm{~ms}$. For each coverslip, four image stacks were acquired.

\subsubsection{Computer analysis of neurite amount}

In order to analyze the amount of neurites, a custom-made "neurite_quantifier" macro was used (Table 3 ). The basic concept of the analysis macro was to determine the location of neurites using a Hessian transformation, which detects line-like structures. In order to avoid detection of non-neuritic structures (such as edges of neuronal cytosols), the cytosolic component of the image was subtracted using the NLS-mCherry signal (Figure 14).

For analysis of neurites, the Cy2 image acquired using $1000 \mathrm{~ms}$ exposure time was selected. After this, a Gaussian blur was applied on the image using the ImageJ "Gaussian Blur" utility. After this, the background of the image was subtracted using the ImageJ "Subtract Background" utility. Following this, the "Tubeness" utility of ImageJ was used, which is a Hessian transformation based analysis method that detects the degree to which structures in the image were "tube-like". The image was then binarized 
using the ImageJ "Threshold" utility, where the image was divided into detected neurites (with a tubeness score over 0 ) and background (tubeness score of 0 ).
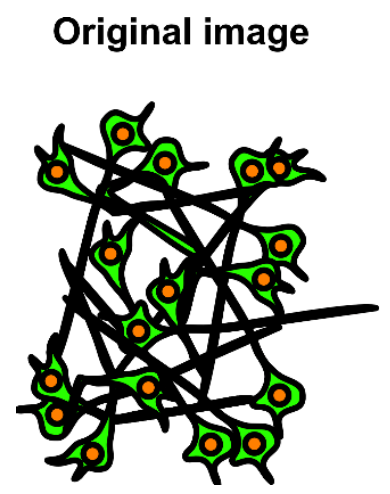

Contours identified using Hessian transformation

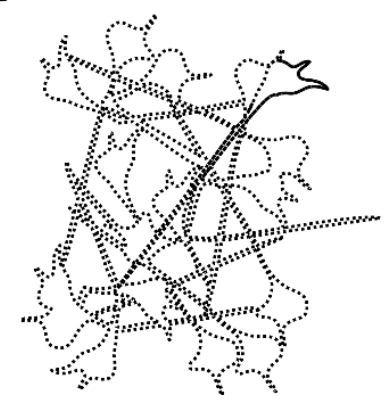

Cytosols subtracted using nuclear signal

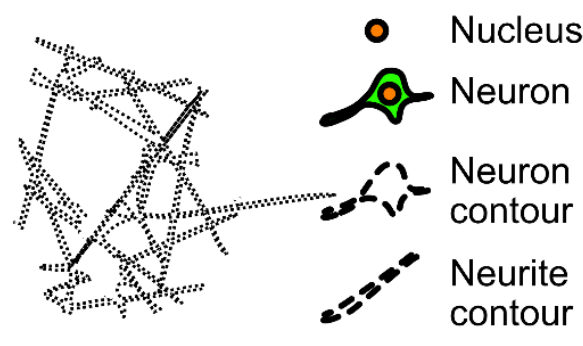

Figure 14. Summary of neurite identification algorithm.

Illustration of the neurite identification algorithm. Depicted are an illustration of an image of neurons (left panel), the contours identified from these neurons using a Hessian transformation (middle panel) and an image of neurite contours after subtraction of the cytosols using the nuclear signal (right panel).

Following this, the NLS-mCherry image acquired using $500 \mathrm{~ms}$ exposure time was selected. Subsequently, this image was segmented to identify the location of cytosols (by identifying the location of the nuclei), for which a method was used that is similar to the one used to segment images for network bursting analysis. The identified cytosols were then subtracted from the binarized image.

Following this, the amount of detected neurites was determined. For this, the size of the area covered by detected neurites was measured, following which the size of the entire area without the subtracted cytosols was measured as well. The percentage of the image area (and thus percentage of well surface) covered by neurites was determined by dividing the first area by the second one.

As mentioned, for each independent culture 4 images had been taken. The values for the 4 images were averaged and the average was recorded as the amount of neurites for that independent culture.

\subsubsection{Immersion microscopy for imaging cAMP levels with AKAR4-cAMPs}

\subsubsection{Microscope setup}

For immersion microscopy, an Axio Examiner D1 microscope was used alongside a set of devices. As light sources, a Colibri LED controller (Zeiss) was used, which permitted illumination of neurons with monochromatic light of specific wavelengths $-360 \mathrm{~nm}, 455 \mathrm{~nm}, 505 \mathrm{~nm}$ and $590 \mathrm{~nm}$ t. As a container for the coverslip during perfusion, a RC-26 GLP imaging chamber (Warner) was used. Importantly, this chamber was modified to ensure removal of the perfusion liquid using a dulled syringe needle. For intermediary storage of the cells before imaging, a C16 Incubator (Labotect) was used.

In order to run the inflow of the perfusion, two Perfusor Secura FT syringe pumps (Braun) equipped with large syringes and $1.57 \times 1.14$ polyethylene tubing (Warner) were used alongside a VC-8 Valve controller (Warner). In order to extract perfusion liquid from the chamber through the dulled syringe needle, a 2120 Varioperpex peristaltic pump (LKB) with 3/16 x 1/8 silicone 3350 tubing (Tygon) was 
utilized. For perfusion liquid, ACSF was used with the following contents $-\mathrm{NaCl}(128 \mathrm{mM}), \mathrm{KCl}(3$ $\mathrm{mM}), \mathrm{MGSO}_{4}(1 \mathrm{mM}), \mathrm{NaH}_{2} \mathrm{PO}_{4}(1 \mathrm{mM})$, HEPES (30 mM), D-Glucose (10 mM), $\mathrm{CaCl}_{2}(1.5 \mathrm{mM}), \mathrm{pH}$ 7.4. The perfusion was run at a speed of $20 \mathrm{ml}$ of liquid passing through the imaging chamber per hour. In order to ensure that the perfusate was at $37^{\circ} \mathrm{C}$ during the imaging, a TC-344B temperature controller (Warner) was used alongside a heated channel which raised the temperature of the perfusate to $37{ }^{\circ} \mathrm{C}$ immediately before it was led into the imaging chamber.

For visualizing the AKAR4-cAMPs signal, a W N-Achroplan 10x 0.3 aperture objective (Zeiss) was used. The neurons were excited with $455 \mathrm{~nm}$ monochromatic light (50\% light intensity) and were passed through a $78 \mathrm{HE}$ filter cube (Zeiss), with the beam divided into one wavelength characteristic of CFP and a second one characteristic of YFP. These beams were then separately picked up using two MRm CCD cameras (Zeiss).

Notably, the NLS-mCherry image was recorded with the same objective. However, the neurons were excited instead with $590 \mathrm{~nm}$ wavelength monochromatic light, which was passed through a $60 \mathrm{HE}$ filter cube (Zeiss).

\subsubsection{Transduction setup (wild-type synucleins, tau protein)}

For measuring the level of cAMP using the AKAR4-cAMPs sensor, neurons were plated on culture plates containing coverslips. After this, neurons were transduced on DIV2 with $3 \times 10^{7}$ AAV virus particles encoding Bcl-xL as well as $1 \times 10^{8}$ AAV virus particles encoding AKAR4-cAMPs. Subsequently, neurons were transduced on DIV4 with $2 \times 10^{8}$ AAV virus particles encoding either a synuclein or tau protein (Figure 8A). The neurons were then incubated until DIV16 or DIV28, after which they were imaged using immersion microscopy.

\subsubsection{Transduction setup ( $\beta$-synuclein P123H mutant)}

For determining the impact of the $\beta$-synuclein P123H mutant, a similar transduction setup was used. However, on DIV4, a different set of synuclein-encoding AAV viruses was used (Figure 8B). Furthermore, on DIV4 the viruses were also transduced with $3 \times 10^{7} \mathrm{AAV}$ virus particles encoding NLSmCherry.

\subsubsection{Recording the AKAR4-cAMPs signal (static)}

A static recording of the neurons was performed for most experiments, such as for the results shown on Figure 27, Figure 31 and Figure 33. For this, the culture plates were extracted from the temporary storage incubator and the coverslips containing the neurons were removed using metal tweezers. The coverslips were placed then into the perfusate which had been previously heated to $37^{\circ} \mathrm{C}$ along with the objective. After focusing, two images stacks were recorded. The first image stack was binned $2 \times 2$ and contained the CFP and YFP components of the AKAR4-cAMPs signal. It was taken at $100 \mathrm{~ms}$ exposure time with the monochromatic light being emitted at a "50\% light intensity" setting. The second image stack was 
binned 2x2, contained several frames of the NLS-mCherry signal and had exposure times ranging from 100 to $8000 \mathrm{~ms}$ with the monochromatic light still being emitted at a "50\% light intensity" setting. It also contained a frame of the CFP signal and an image acquired using brightfield illumination.

\subsubsection{Recording the AKAR4-cAMPs signal (dynamic)}

Notably, for Figure 7, a dynamic recording of AKAR4-cAMPs was performed. For this, the images were acquired using the same settings as for the static recording. However, instead of taking a single image, an 800 second video was taken instead at a sampling rate of 1 frame per second. The same objective, exposure time, monochromatic light and light intensity was used.

During this imaging, the perfusion solution was exchanged twice - once to apply an ACSF dilution containing linderane $(20 \mu \mathrm{M})$ and SQ-22536 $(20 \mu \mathrm{M})$ and a second time to apply an ACSF dilution containing forskolin $(5 \mu \mathrm{M})$ and rolipram $(5 \mu \mathrm{M})$ at times indicated in the figure (Figure 7$)$. In order to perform this exchange, the two syringe pumps were loaded with a syringe, with one of them containing the original ACSF perfusate and the other one containing the dilution. At the moment of the exchange, the first syringe pump was switched off and the second was switched off. Furthermore, the perfusion speed was increased to $80 \mathrm{ml}$ per hour during the first 30 seconds of the exchange to ensure more rapid exchange of the perfusates.

\subsubsection{Image analysis}

Subsequently, the image was processed using custom-made ImageJ macros -"mCherry_segmentation", "akar_divider" and "scanner" (Table 3). The "mCherry_segmentation" macro was the same as the one used for the network burst and neurite amount analysis and served the purpose of identifying cytosol locations via identification of nuclear locations. The second macro, "akar_divider" was used to create a ratio image from the CFP and YFP (FRET) components of the AKAR4-cAMPs signal. This was achieved by dividing the YFP component with the CFP component using the ImageJ "Image Calculator" utility.

The third macro, "scanner" used the file containing the cytosol locations from the "mCherry_segmentation" macro and the YFP/CFP ratio as inputs. In terms of its function, it served to scan the ratio image at each of the cytosol locations using the ImageJ "multi-measure" and "Measure" functions. The YFP/CFP ratio of all neurons in the image was then averaged and stored for statistical analysis.

\subsubsection{Immersion microscopy for imaging cAMP levels with cAMPr}

\subsubsection{Microscope setup}

The same microscope equipment and perfusion setup was used to record cAMPr as was used to record AKAR4-cAMPs. With regard to the optical settings, $455 \mathrm{~nm}$ monochromatic light was used to excite cAMPr, which was then passed through a 78 HE filter cube (Zeiss). Identically to AKAR4-cAMPs, a 
W N-Achroplan 10x 0.3 aperture objective (Zeiss) was used. Importantly, when using the cAMPr sensor the cAMPr signal was picked up using only one camera, not two as was done for the AKAR4-cAMPs signal.

\subsubsection{Transduction setup (wild-type synucleins, tau protein)}

In order to measure the level of cAMP using the cAMPr sensor, neurons were plated on culture plates containing coverslips. Then, neurons were transduced on DIV2 with $3 \times 10^{7}$ AAV virus particles encoding Bcl-xL as well as $1 \times 10^{8} \mathrm{AAV}$ virus particles encoding cAMPr. Subsequently, neurons were transduced on DIV4 with $2 \times 10^{8} \mathrm{AAV}$ virus particles encoding either a synuclein or tau protein (Figure 8A). The neurons were then incubated until DIV16 or DIV28, after which they were imaged using immersion microscopy.

\subsubsection{Transduction setup ( $\beta$-synuclein P123H mutant)}

In order to determine the impact of the $\beta$-synuclein $\mathrm{P} 123 \mathrm{H}$ mutant, a similar transduction setup was used. However, on DIV4, a different set of synuclein-encoding AAV viruses was used (Figure 8B). Furthermore, on DIV4 the viruses were also transduced with $3 \times 10^{7} \mathrm{AAV}$ virus particles encoding NLSmCherry.

\subsubsection{Recording the cAMPr signal (static)}

A static recording of neurons expressing cAMPr was performed for the experiment shown on Figure 27. For acquiring this image, the culture plates were removed from the temporary storage incubator and the coverslips containing the neurons were extracted using metal tweezers. These were placed then into perfusate which had been earlier heated to $37^{\circ} \mathrm{C}$ along with the objective. After focusing the image, two image stacks were recorded. The first image stack contained a single cAMPr image. It was taken at 500 ms exposure time after neurons were excited with monochromatic $455 \mathrm{~nm}$ light being emitted at a "100\% light intensity" setting. The second image stack was binned $2 \times 2$, contained several frames of the NLSmCherry signal and had exposure times ranging from 100 to $8000 \mathrm{~ms}$ with the monochromatic light being emitted at a "50\% light intensity" setting. It also contained a frame of the CFP signal and an image acquired using brightfield illumination.

\subsubsection{Recording the cAMPr signal (dynamic)}

Notably, a dynamic recording of neurons expressing cAMPr in the presence of various molecular factors was performed for the experiment shown on Figure 6. This recording was performed similarly as the dynamic recording for neurons expressing AKAR4-cAMPs, with a 1000 second video being recorded at a sampling rate of 1 frame per second. The key difference was the imaging settings - each frame for the cAMP recording was gathered with an exposure time of $500 \mathrm{~ms}$ with the monochromatic $455 \mathrm{~nm}$ light being emitted at a "100\%" light intensity setting. 


\subsubsection{Image analysis}

In order to analyze the cAMP signal recordings, the custom-made ImageJ macros "mCherry_segmentation" and "scanner" were used. The "mCherry_segmentation" functioned identically as for the network burst analysis and was used to determine the location of neuronal cytosols via location of nuclei.

Meanwhile, the "scanner" macro used the neuronal cytosol location as an input in addition to the static recording of cAMPr fluorescence. Using this images, it scanned the cAMPr fluorescence image at each of the cytosol locations using the ImageJ "multi-measure" and "Measure" utilities. The recorded cAMPr fluorescence all neurons in the image was then averaged and stored for statistical analysis. 


\section{Results}

Previous studies have indicated that in both human patients and experimental animals, increased levels of $\alpha$-synuclein interfere with rhythmic neuronal network activity, which is a type of activity involving repeated depolarizations of neuronal networks ${ }^{13,133,134}$. However, not much is known about the mechanisms leading to this phenomenon on a cellular level, which are difficult to study in vivo due to the difficulty of assaying impacted neurons.

To counteract this limitation, this study used cultured neurons to model the ways in which increased levels of $\alpha$-synuclein interfere with rhythmic neuronal network activity. This model was selected due to two factors. Firstly, the extracellular environment of neurons could be manipulated and analyzed more easily in vitro than in vivo, allowing for the study of extracellular $\alpha$-synuclein species, which have been shown to interfere with neuronal function in a variety of ways ${ }^{136,271-273}$. Secondly, using this model allowed for the easier application of several experimental methods, such as fluorescent microscopy and immunocytochemical staining.

The overall plan of the study was divided into three steps. Firstly, it was determined whether $\alpha$-synuclein overexpression changed the behavior of network bursts, which is the characteristic rhythmic activity of cultured neurons. Secondly, if this reduction was observed, the model was used to study the cellular mechanisms that triggered these changes. Thirdly, the model was used to determine whether the neuron network would be impacted by the overexpression of other disease-associated proteins, such as mutant species of $\beta$-synuclein and tau protein.

\subsection{Optimization of the experimental setup}

\subsubsection{Minimizing in vitro cell death}

However, before any experiments could be performed, it was necessary to minimize levels of cell death in the cultured neuron model. This was important to ensure the presence of network bursting, which according to a study by Ito et al., required a culture density of more than 50 neurons $/ \mathrm{mm}^{2}$. Notably, in one of our previous studies, cultures were observed to drop to this density (50 neurons $/ \mathrm{mm}^{2}$ ) and also below it when expressing proteins that induced cell death, such as $\alpha$-synuclein ${ }^{78}$. Furthermore, such levels of cell death were observable already at DIV18, only 4 days after DIV14, the timepoint on which network bursting becomes mature in vitro ${ }^{78,116}$.

Additionally, minimization of cell death was required to study how sample proteins impacted the functioning and structural integrity of neuronal structures, such as axons, dendrites and synapses, independently of cell death. This was important as previous studies had shown that the density of such structures in culture (e.g. neurite density) was correlated with the density of neurons per $\mathrm{mm}^{2}{ }^{18}$. 
In order to reduce levels of cell death, two methods were applied. Firstly, neurons were induced to overexpress Bcl-xL, which had previously been shown to be an effective inhibitor of neuronal cell death 113,274. Secondly, the water vapor levels inside the neuron culture incubator were saturated, which was done to minimize evaporation. This was necessary as evaporation of water changes the osmolarity of the culture medium, which is known to reduce the viability of cultured neurons ${ }^{275,276}$. In order to reduce evaporation, the culture incubator was changed from a HeraCell incubator (Heraeus) to a Labo C201 incubator (Labotect), which enables the active regulation of humidity levels inside the incubator.

In order to test the whether these two methods reduced cell death, neuron cultures were induced to overexpress $\alpha$-synuclein from DIV4 to DIV28 in three experimental groups. The first group was induced to overexpress Bcl-xL and was cultured in the HeraCell incubator, whereas the second group was induced to overexpress with Bcl-xL and was also cultured in the HeraCell incubator. Finally, the third group was induced to overexpress Bcl-xL and was cultured in the humidifying Labo C201 incubator. When comparing the number of surviving neurons at DIV28 between these three groups, cell survival was significantly greater in both of the groups overexpressing Bcl-xL. More specifically, 410-450 neurons per $\mathrm{mm}^{2}$ survived in cultures overexpressing Bcl-xL, whereas only 54 neurons per $\mathrm{mm}^{2}$ survived in cultures not overexpressing Bcl-xL (Figure 15).Because as many as $410-450$ neurons $/ \mathrm{mm}^{2}$ neurons survived in the two experimental groups overexpressing Bcl-xL, these two groups were further tested to determine whether these cultures exhibited network bursting. In order to analyze network bursting, neurons were induced to overexpress Bcl-xL, $\alpha$-synuclein and the calcium sensor GCaMP6. One group was cultured inside a HeraCell incubator, whereas a second group was cultured inside the humidifying Labo C201 incubator. In order to characterize the network bursts, the frequency of bursts per minute was determined. It was thus observed that on DIV28, both cultures exhibited network bursting. However, the average frequency of this activity was significantly higher in neurons cultured in the humidifying Labo C201 incubator (3.4 network bursts per minute) than in the HeraCell incubator (1.3 network bursts per minute) (Figure 15).

Based on this result, the condition where neurons were induced to overexpress Bcl-xL and were cultured in the humidifying Labo C201 incubator was selected for all subsequent experiments as the standard experimental setup. As a final test, it was determined whether the network bursting of such cultures evolved in the same way as described in literature, with a maturation of the culture characterized by an increase in culture synchronization around DIV14-15. To perform this test, neurons were induced to overexpress Bcl-xL, GCaMP6 (to detect network bursts) and the control protein $\gamma$-synuclein. In order to analyze the level of synchronization, the amount of neurons involved in network bursting (as \% of total neurons) was determined. As expected, this amount increased significantly from DIV10 to DIV16, indicating a maturation of the neuron culture between these two timepoints. Interestingly, the amount of neurons involved in network bursting declined significantly from DIV16 to DIV28. This was likely due to culture aging, as synchronized network bursting ceased by DIV39 (Figure 16). 
A

Impact of culture optimization: Number of neurons

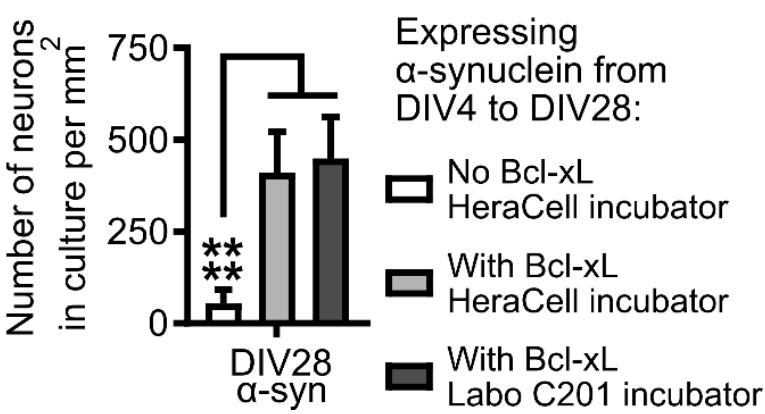

B Impact of culture optimization: Frequency of network bursts

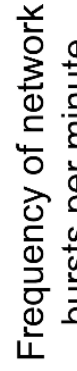
Expressing a-synuclein from DIV4 to DIV28:

With Bcl-xL HeraCell incubator

With Bcl-xL Labo C201 incubator

Figure 15. Culture optimization reduces cell death and increases the frequency of rhythmic activity.

(A) Quantification of culture density neurons in cultures that expressed $\alpha$-synuclein from DIV4 to DIV28 under three separate conditions - neurons not expressing Bcl-xL that were incubated in a HeraCell incubator (white bars), neurons overexpressing Bcl-xL that were incubated in a HeraCell incubator (light gray bars) and neurons overexpressing Bcl-xL that were incubated in a humidifying Labo C201 incubator (dark gray bars). The number of neurons in culture per $\mathrm{mm}^{2} \pm$ standard deviation is shown. Statistical analysis: two-tailed t-test with Welch's correction and a post-hoc test to determine statistical power. $* * * *$ : $p<0.0001$. Statistical power: $1-\beta>100 \%$. Sample size of independent cultures: 6 (white bars), 9 (light gray bars), 12 (dark gray bars).

(B) Quantification of the frequency of network bursts in neurons expressing $\alpha$-synuclein from DIV4 to DIV28 and Bcl-xL from DIV2 under two conditions - neurons cultured in a HeraCell incubator (Heraeus) (light gray bars) and neurons cultured in a humidifying Labo C201 incubator (Labotect) (dark gray bars). The number of network bursts per minute \pm standard deviation is shown. ${ }^{* *}: \mathrm{p}<0.01$. Statistical power: $1-\beta>84 \%$. Sample size of independent cultures: 9 (light gray bars), 12 (dark gray bars).

\section{Evolution of neuron culture synchronization}

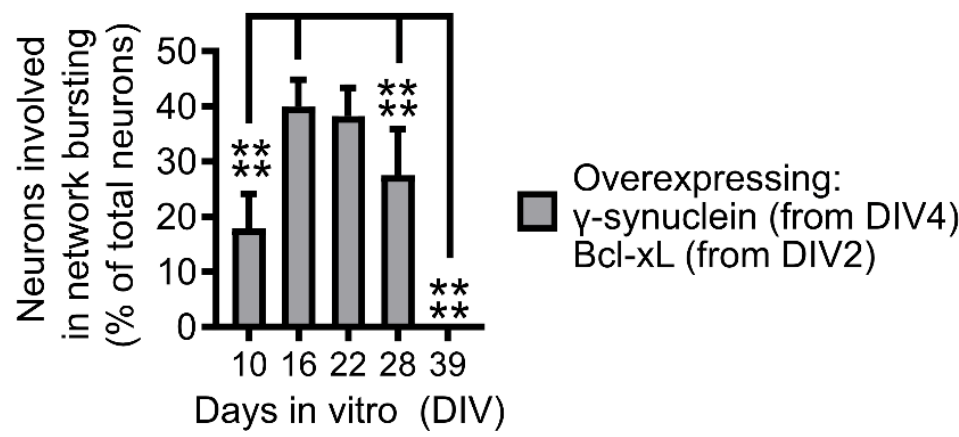

Figure 16. The synchronization of neuron network bursting increases from DIV10 to DIV16 and then declines.

Quantification of the amount of neurons involved in network bursting in cultures overexpressing $\gamma$-synuclein and Bcl-xL. The number of neurons involved in bursting (as \% of total neurons) \pm standard deviation is indicated. Statistical analysis was performed using a one-way ANOVA alongside a post-hoc power analysis. $* * * *$ : $p<0.0001$. Statistical power: $1-\beta>100 \%$. Sample size of independent cultures: 18 .

\subsubsection{Selection of methodology}

After this, the next task was to select the experimental parameters. To begin, it was necessary to determine the experimental readout that would be used to characterize the properties of neuron network bursting. Importantly, previous studies had observed that more sizable levels of $\alpha$-synuclein increase the prevalence of low frequency rhythmic activity in vivo ${ }^{13,133}$. As these studies had characterized rhythmic activity in terms of its frequency, the frequency of in vitro rhythmic activity (i.e. frequency of network bursts) was thus selected as the primary readout in our study as well. Importantly, this was done in 
acknowledgement of the fact that network bursts are far simpler than in vivo types of rhythmic activity observed in the brain, which involve several brain regions and many different activity types ${ }^{86,110,118}$.

Subsequently, it was necessary to also select a readout that could be used as a simple indicator of neuronal connectivity, as this had previously been shown to be one of the key parameters determining the behavior of neuronal networks ${ }^{16-20}$. To achieve this purpose, the amount of neurons involved in network bursting was selected as the secondary readout. It was reasoned that a presence of broken neuronal connections would prevent the depolarizing impulse of the network burst from reaching additional neurons, which would thus lead to a reduction in the number of neurons involved in network bursting.

After selecting the experimental readouts, it was necessary to determine the method that would be used to overexpress $\alpha$-synuclein and the other experimental proteins. Notably, in the study by McDowell et al., overexpression of $\alpha$-synuclein had been achieved by genetically modifying animals to contain a human $\alpha$-synuclein gene under the control of the Thy 1 promoter ${ }^{133}$. In our study, this method was ruled out, as it was necessary to overexpress more than one protein in order to establish our experimental model (e.g. control synucleins, tau protein, genetically-encoded sensors). Therefore, the experimental proteins were instead expressed by transducing neurons with AAV6 viral particles ${ }^{113}$. This was done as the AAV6 transduction method allowed us to express several different proteins and also to generate new expression vectors. Beyond this, the AAV6 method was selected as it had been used for neuronal expression in our research previously, including for researching the impact of $\alpha$-synuclein ${ }^{78,113,250}$.

Notably, our cultures were neuron-glia co-cultures, where glia were included to ensure neuronal maturation and maintenance ${ }^{276-279}$. As a result, it was necessary to ensure that expression would be neuron-specific, for which all proteins were expressed under the control of a neuron-specific hSyn promoter, which was placed in front of the cDNA sequence in the AAV expression cassette (Figure 8) 250,278. Ensuring neuron specificity was relevant, as the focus in this study was on the impact of $\alpha$-synuclein on the physiology of neurons, not glial cells .

Notably, the study by McDowell et al. observed that $\alpha$-synuclein disrupted rhythmic activity in 10 month old animals when using a model that increased $\alpha$-synuclein expression $2-5$ fold ${ }^{131,133}$. As our cultures exhibited rhythmic activity for only around 5 weeks, it was essential to increase the expression level beyond what was used by McDowell et al. to ensure that any rhythmic activity disruptions of $\alpha$-synuclein would be observable inside the 5 week timeframe. In order to achieve this, expression-boosting regulatory sequences were included in most of the constructs, such as the WPRE and bGH pA elements (Figure 8).

After determining the means of protein expression, it, was necessary to select a negative control for $\alpha$-synuclein. Notably, our previous studies had used fluorescent proteins (e.g.) EGFP as controls, which was however problematic due to these proteins not being similar to synucleins in terms of structure ${ }^{78,113}$. 
Therefore, it was necessary to select a new negative control that was structurally and phylogenetically related to $\alpha$-synuclein but less toxic and disruptive in terms of its impact. For this role, the other two members of the synuclein family were considered $-\beta$-synuclein and $\gamma$-synuclein. Of the two, $\beta$-synuclein was similar in structure to $\alpha$-synuclein but had been described to induce neuronal cell death in various articles and thus was unsuitable as a negative control ${ }^{78,84}$. In contrast, $\gamma$-synuclein was also similar in structure to $\alpha$-synuclein but had not been described to be toxic and was thus selected as the primary negative control.

Finally, it was necessary to decide which method would be used for the measurement of network bursting. Notably, previous studies of network bursting had used multielectrode arrays to measure network bursting, which has the advantage of measuring membrane potential changes directly ${ }^{280,281}$. However, this method was not selected in our study, as it is associated with characteristic problems, such as the difficulty of determining how many neurons produce the signals detected at the electrode and where these neurons are located ${ }^{124}$. As a result, network bursts were instead visualized using the GCaMP6 genetically encoded calcium sensor, which would detect the network bursts by identifying the post-depolarization calcium influx (Figure 4) ${ }^{86,113,118,125}$.

\subsubsection{Determining the synuclein expression level}

After selecting the experimental methodology, the expression rate of $\alpha$-synuclein for all subsequent experiments was determined. As mentioned, the intention was to choose the highest possible expression rate, as that would increase the likelihood of detecting neuronal network alterations using $\alpha$-synuclein during the five week lifespan of the culture. However, it was also considered important to ensure that the expression rate was not innately toxic to the neurons - although AAV6 viruses have a low toxicity, they can be toxic when very high amounts (e.g. over $10^{10}$ viral genome copies) are used ${ }^{282}$.

In order to determine the highest non-toxic level of expression, an experiment was designed where neuron cultures were induced to overexpress $\gamma$-synuclein using different amounts of viral particles$4 \times 10^{8}, 2 \times 10^{8}, 1 \times 10^{8}$ and $5 \times 10^{7}$ viral particles. The cultures also expressed Bcl-xL and GCaMP6 for measuring the frequency of network bursts. Using this experimental setup, it was determined that the frequency of network bursts was significantly reduced when $\gamma$-synuclein overexpression was induced using $4 \times 10^{8}$ as opposed to $2 \times 10^{8}$ viral particles, indicating that the higher expression level disrupted neuron network bursting. However, the frequency of network bursts was not altered when $\gamma$-synuclein overexpression was induced by $2 \times 10^{8}$ as opposed to $1 \times 10^{8}$ and $5 \times 10^{7}$ viral particles (Figure 3 ). Thus protein expression using $2 \times 10^{8}$ viral particles were determined to be the highest expression rate that did not disrupt neuron network bursting. This expression rate was therefore used in all subsequent experiments to overexpress synucleins and tau protein.

After determining the expression rate for $\alpha$-synuclein overexpression, it was necessary to ensure that the expression level of the $\alpha$-synuclein and $\gamma$-synuclein expressing viruses was equivalent to each other. 
This was done to ensure that any observed disruption of neuron network activity would be caused by the unique impacts of the overexpressed synuclein, not by a difference in the amount of viral particles used to trigger the overexpression. In order to determine whether the viral expression amounts were equal, the level of NLS-mCherry expressed by the viruses was compared, as all synuclein expressing viruses also induced the expression of NLS-mCherry (Figure 8). Doing this, comparable levels of expression were observed for the vector used for expressing $\alpha$-synuclein as for the one used for expressing $\gamma$-synuclein (Figure 17).

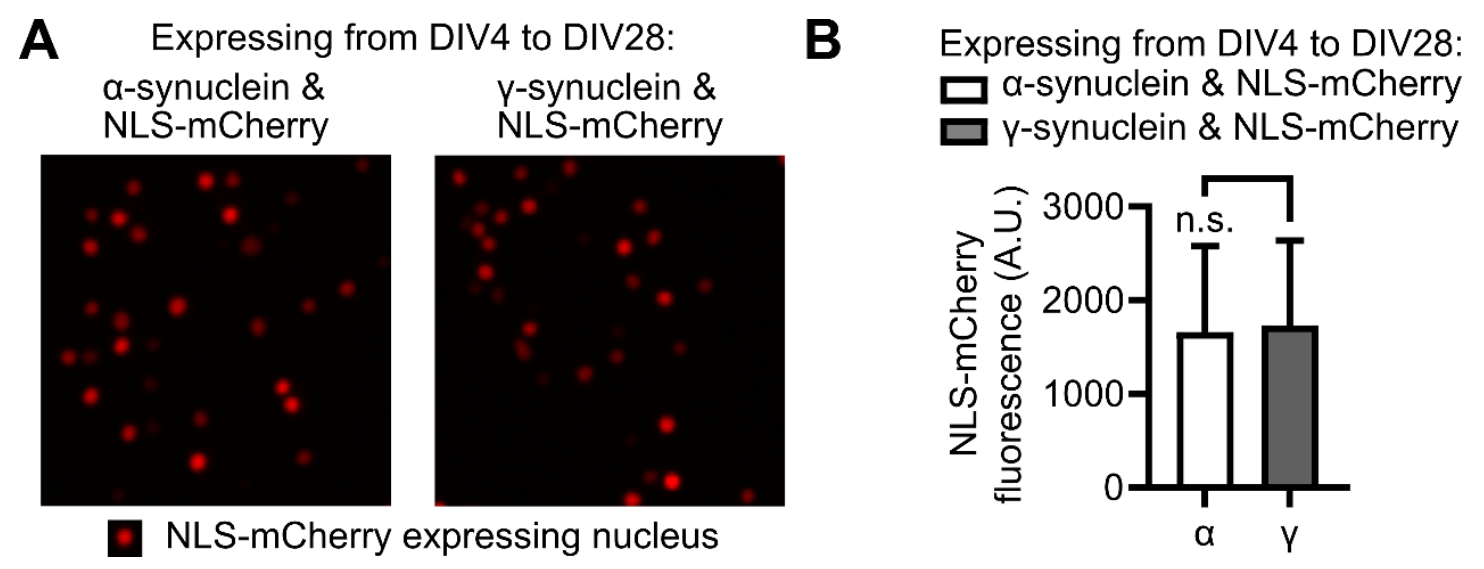

Figure 17. $\alpha$-synuclein and $\gamma$-synuclein expression levels are equal when induced using $2 \times 10^{8}$ AAV viral particles.

(A) Micrographs of neurons that were induced to express either $\alpha$-synuclein or $\gamma$-synuclein from DIV4 using $2 \times 10^{8}$ AAV viral particles. Both vectors also contained the cDNA for NLS-mCherry (Figure 8), so the expression of NLS-mCherry (red) is depicted. NLS-mCherry = nuclear localization signal tagged mCherry.

(B) Average absolute fluorescence of neurons expressing synuclein alongside NLS-mCherry. Average fluorescence \pm standard deviation is depicted. "A.U." = arbitrary units. Statistical analysis: two-tailed t-test with Welch's correction and a post-hoc power analysis. "n.s." = not significant. Sample size: 100 neuronal nuclei (per condition).

\section{2. $\alpha$-synuclein overexpression reduces the frequency of neuron network bursting in culture}

After the experimental setup had been optimized, it was necessary to show that $\alpha$-synuclein overexpression reduced the frequency of rhythmic activity in vitro, just as increased levels of $\alpha$-synuclein altered the frequency of rhythmic activity in vivo. This was required, as the prepared in vitro setup was suitable as a model for the in vivo phenomenon only if such a reduction was observed. Indeed this was the case, as neurons overexpressing $\alpha$-synuclein had a significantly reduced burst frequency compared to neurons overexpressing the control protein $\gamma$-synuclein. From DIV15-22, a small reduction of $-14 \%$ to $-16 \%$ was observed, with this reduction being significant at DIV15. In comparison, from DIV25 to DIV31, the reduction was more pronounced and grew larger over time, with the reduction of burst frequency between $\alpha$-synuclein and $\gamma$-synuclein overexpressing neurons increasing from DIV25 $(-45 \%)$ to DIV31 (-74\%) (Figure 18).

Interestingly, the reduction in frequency by $\alpha$-synuclein consisted of two distinct impacts on neuron network functioning. Firstly, $\alpha$-synuclein overexpression triggered a small but marginally significant 
reduction of burst frequency between DIV15 and DIV22. Secondly, $\alpha$-synuclein overexpression induced a much larger impact from DIV25 to DIV31 (Figure 18). This was likely caused due to the aging of the culture, as the larger impact emerged 1-2 weeks before the end of synchronized network bursting at DIV39 (Figure 16).

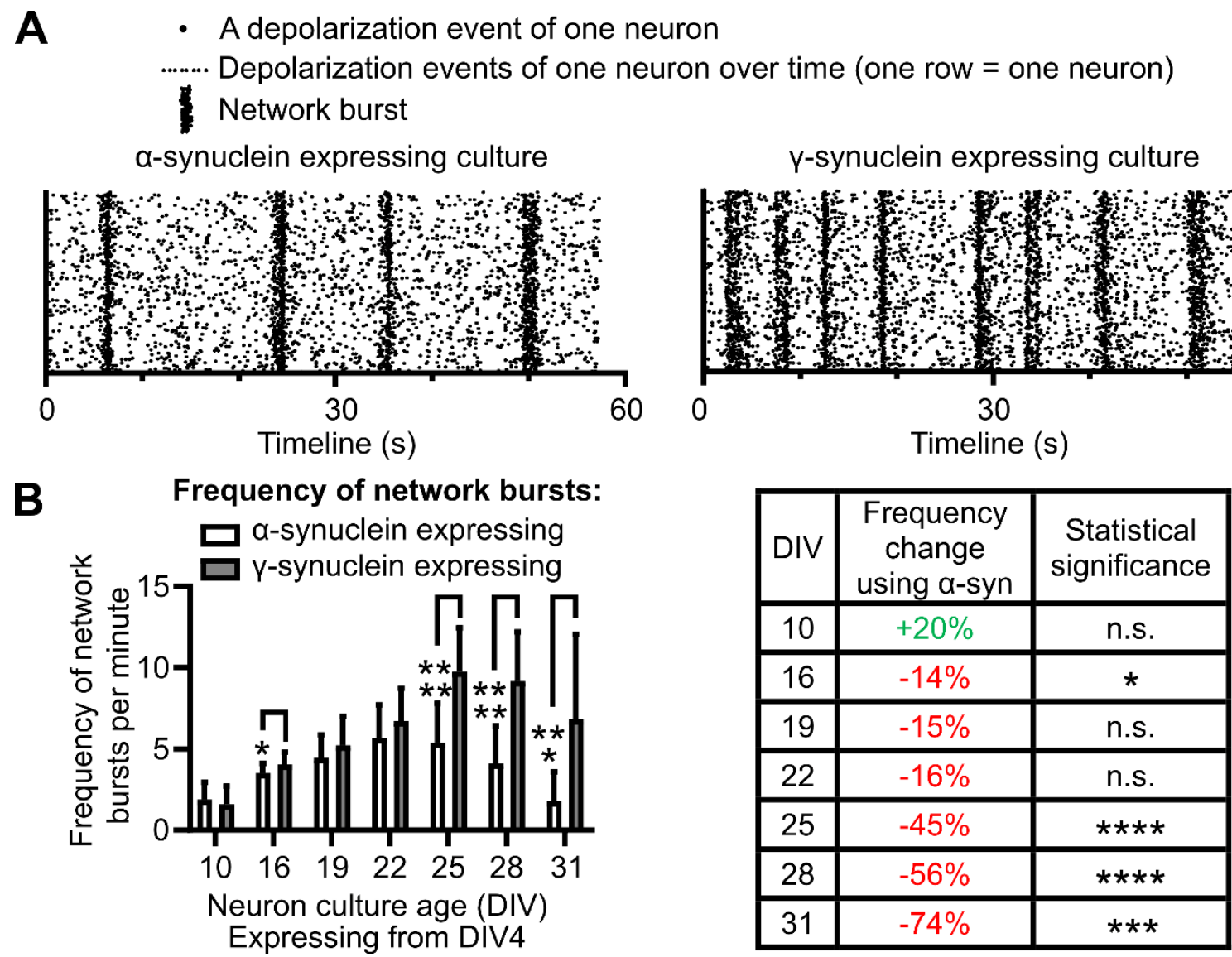

Figure 18. $\alpha$-synuclein overexpression reduces the frequency of network bursting in cortical culture.

(A) Scatter plot demonstrating non-stimulated network bursting for cultures overexpressing either $\alpha$-synuclein (left panel) or $\gamma$-synuclein (right panel) at DIV28. Dots aligned in a row $=$ depolarization events by an individual neuron. (B) (Left panel) Quantification of network burst frequency in $\alpha$-synuclein overexpressing neurons (white bars) and $\gamma$-synuclein overexpressing neurons (gray bars). The number of network bursts per minute \pm standard deviation is shown. (Right panel) Table showing the percentage reduction of burst frequency inside neurons overexpressing $\alpha$-synuclein when compared to neurons overexpressing $\gamma$-synuclein. Statistical analysis: two-tailed t-test with Welch's correction and a post-hoc power analysis. "n.s." = not significant. $*$ : p < 0.05 , ****: p $<0.0001$. Statistical power: DIV15: $1-\beta>67.2 \%$, DIV25: $1-\beta>99.9 \%$, DIV28: $1-\beta>100.0 \%$, DIV31: $1-\beta>96.5 \%$, sample size: 18 independent cultures.

After this, it was analyzed whether overexpression of $\alpha$-synuclein caused greater levels of cell death than the overexpression of control protein $\gamma$-synuclein. This was done to identify whether the observed reduction in burst frequency was caused by death of cultured neurons or by a disruption of neuron physiology not directly related to cell death (e.g. through a loss of neuritic structures or synapses). To quantify whether $\alpha$-synuclein caused increased levels of cell death, the number of neurons was quantified at the same location from DIV10-DIV32. This revealed that the same percentage of neurons survived in cultures overexpressing $\alpha$-synuclein as in cultures overexpressing $\gamma$-synuclein (Figure 19). 


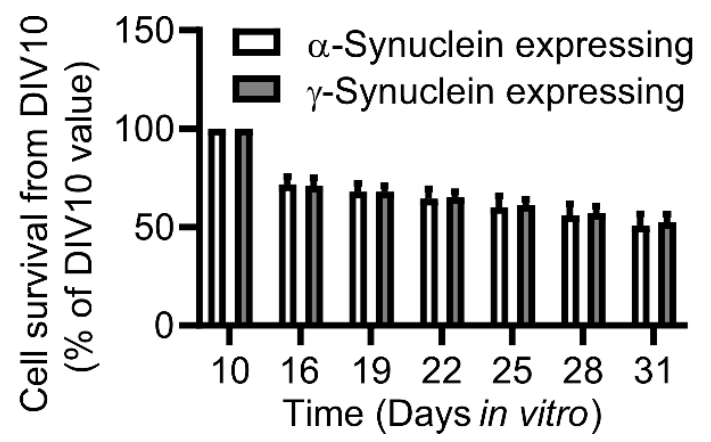

Figure 19. $\alpha$-synuclein overexpression does not induce additional cell death when expressed in neurons alongside anti-apoptotic factor Bcl-xL.

Quantification of neuron survival in neurons overexpressing either $\alpha$-synuclein (white bars) or $\gamma$-synuclein (gray bars) in the presence of Bcl-xL overexpression. The number of neurons surviving from DIV10 (as \% of the number of neurons for that condition at DIV10) \pm standard deviation is depicted. Statistical analysis: two-tailed t-test with Welch's correction and a post-hoc test for statistical power. Sample size: 18 independent cultures.

A

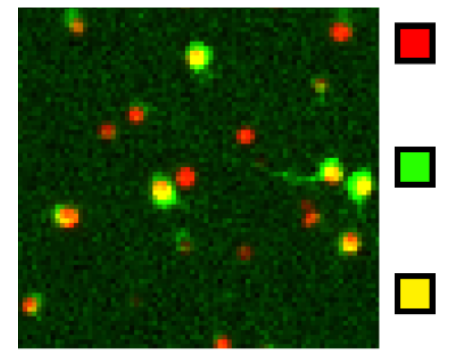

Nuclei

$\mathrm{Ca}^{2+}$ response during a burst

Merge

Neurons not involved in bursting

* Neurons involved in bursting

\section{B \% of neurons involved in bursting:}

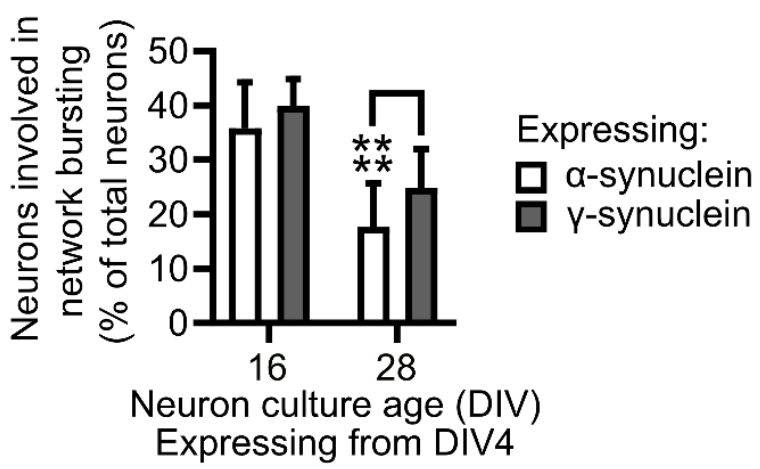

Figure 20. $\alpha$-synuclein overexpression reduces the amount of neurons involved in neuron network bursting. (A) Fluorescent image of neuron calcium response during a network burst. Cell nuclei = red (indicator - NLSmCherry), calcium sensor response = green (indicator - GCaMP6), merge of two signals = yellow.

(B) Quantification of the amount of neurons involved in network bursting in $\alpha$-synuclein overexpressing neurons (white bars) or $\gamma$-synuclein overexpressing neurons (gray bars). The average amount of neurons involved in a network burst (as \% of total neurons) \pm standard deviation is displayed. Statistical analysis: Two-tailed t-test with Welch's correction, post-hoc statistical power test. $* * * *: p<0.0001$. Statistical power of DIV28 comparison: $1-\beta$ $>$ 99\%. Sample size: 41 independent cultures.

Subsequently to this, it was investigated whether $\alpha$-synuclein was interfering with the connectivity of the neuron network, as this would be a possible explanation for the observed reduction of neuron network burst frequency. As stated earlier, it was reasoned that a disrupted neuronal connectivity would prevent the depolarizing impulse of the network burst from reaching additional neurons and would therefore reduce the amount of neurons involved in neuron network bursting. Indeed, overexpression of $\alpha$-synuclein significantly reduced the amount of neurons involved in neuron network bursting by $29 \%$ when compared to $\gamma$-synuclein overexpressing neurons (Figure 20). This therefore suggested that $\alpha$-synuclein indeed interfered with the connectivity of neurons. 


\subsection{The cell culture supernatant of neurons overexpressing $\alpha$-synuclein contains up to $0.36 \mu \mathrm{M}$ released extracellular $\alpha$-synuclein.}

Our experiments therefore showed that the frequency of network bursts and the amount of neurons involved in neuron network bursting were reduced by $\alpha$-synuclein overexpression. However, it remained unclear as to what amount of $\alpha$-synuclein had triggered this reduction and whether that $\alpha$-synuclein was intracellular or extracellular. It was thus decided to determine the amount of $\alpha$-synuclein both in the cell culture supernatant (for extracellular synuclein) and in cell lysates (for intracellular synuclein). Determining this was important, as it would further explain why $\alpha$-synuclein overexpression reduced the frequency of network bursting. This was done under the premise that if the cell culture supernatant was observed to contain large amounts of $\alpha$-synuclein, then this would be a suggestion of a possible role of extracellular $\alpha$-synuclein in reducing the frequency of neuron network bursting.

In order to determine this, an experiment was designed to identify the amount (in $\mu \mathrm{g}$ ) and concentration of extracellular and intracellular $\alpha$-synuclein. Briefly, neurons expressed $\alpha$-synuclein from DIV4 onward - then, on DIV10, DIV16, DIV22 and DIV28, the cell culture supernatant of the neurons was collected to determine the level of extracellular $\alpha$-synuclein and lysates of the cells were gathered for determining the level of intracellular $\alpha$-synuclein. The mass of $\alpha$-synuclein was then determined by Western Blot, where the lysate and medium samples were loaded alongside 4-5 lanes of a commercial $\alpha$-synuclein standard with a known mass. For determining the mass, the standards were used to create a calibration curve, which described the relationship between the intensity of the $\alpha$-synuclein bands and the mass of $\alpha$-synuclein. This curve was then used to calculate the mass of the sample bands (Figure 11).

After determining the mass of the Western blot samples, the total mass of extracellular and intracellular $\alpha$-synuclein in the culture or lysate was calculated. This was possible, as for loading the samples, a known fraction of the total lysate (e.g. $1 \%$ of the total volume) had been used. The mass of this fraction could thus be multiplied (e.g. multiplied 100 times for a $1 \%$ fraction) to determine the total mass of $\alpha$-synuclein in the whole lysate or medium (in $\mu \mathrm{g}$ ).

Beyond determining the mass of $\alpha$-synuclein, the intra- and extracellular synuclein concentration of the samples was also determined. This was done to compare the molar concentration of $\alpha$-synuclein with previously published data. For determining the molar concentration of the samples, a formula was used, where the concentration could be determined based on the calculated mass, the molar mass of $\alpha$-synuclein and either the volume of the culture medium or the total volume of the neurons (Figure 12). Notably, the volume of the culture medium was determined to be 625-720 $\mu 1$ depending on the timepoint. For determining the volume of the neurons, the total number of neurons at the various timepoints was experimentally determined, following which the number was multiplied with a value for individual neuron volume derived from previous publications, i.e. $6 \mathrm{pl}$ per neuron ${ }^{266-268}$. Doing this, the total 
volume of the cells was calculated to be $0.5-1.0 \mu 1$ depending on the total number of neurons, which varied from 450 to 900 cells per $\mathrm{mm}^{2}$ depending on the timepoint and the experimental condition.

This procedure was thus carried out to determine the amount of extracellular and intracellular $\alpha$-synuclein. When this was done, it was observed that the amount of intracellular $\alpha$-synuclein increased from $7 \mu \mathrm{g}(0.5 \mathrm{mM})$ at DIV10 to $10.5 \mu \mathrm{g}(1.4 \mathrm{mM})$ at DIV28, plateauing at DIV22. Meanwhile, the amount of extracellular $\alpha$-synuclein was lower than intracellular $\alpha$-synuclein and increased from $0.2 \mu \mathrm{g}$ $(0.02 \mu \mathrm{M})$ at DIV10 to $3.3 \mu \mathrm{g}(0.36 \mu \mathrm{M})$ at DIV28, with this value also plateauing at DIV22 (Figure 21). Although the cause of the plateauing could not be determined exactly, it was explainable in light of previous studies, which indicated that the expression rate induced by AAV viruses could level off at later timepoints ${ }^{283}$. Interestingly, the most rapid increase happened from DIV10 to DIV16, with the amount of extracellular $\alpha$-synuclein increasing around 8 fold $(0.2 \mu \mathrm{g}$ to $1.7 \mu \mathrm{g})$ (Figure 21$)$. To explain this rise, it is possible that increased levels of $\alpha$-synuclein were released after initiation of mature network bursting around DIV16, as increased neuronal activity is thought to be one of the parameters determining the release rate of $\alpha$-synuclein ${ }^{284}$.
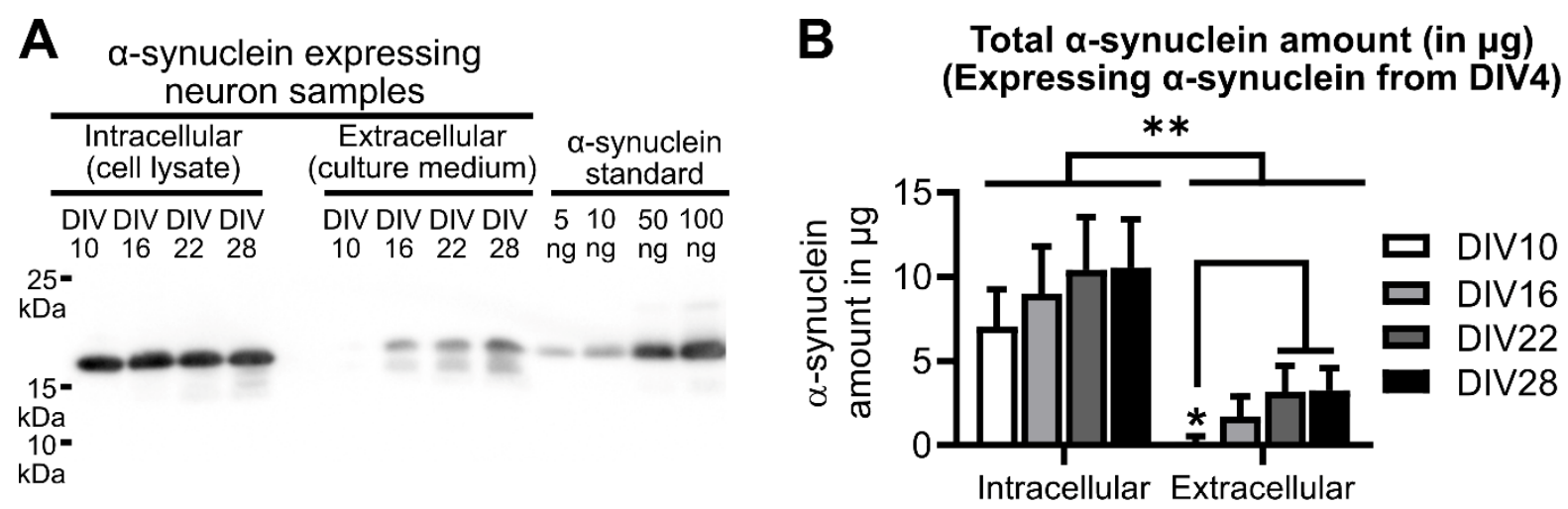

\begin{tabular}{|c|c|c|c|c|}
\hline 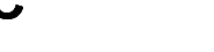 & $\overline{\text { DIV10 }}$ & $\overline{\text { DIV16 }}$ & $\overline{\text { DIV22 }}$ & $\overline{\text { DIV28 }}$ \\
\hline Intracellular & $7 \mu \mathrm{g} ; 0.5 \mathrm{mM}$ & $9 \mu \mathrm{g} ; 0.9 \mathrm{mM}$ & $10.4 \mu \mathrm{g} ; 1.1 \mathrm{mM}$ & $10.5 \mu \mathrm{g} ; 1.4 \mathrm{mM}$ \\
\hline Extracellular & $0.2 \mu \mathrm{g} ; 0.02 \mu \mathrm{M}$ & $1.7 \mu \mathrm{g} ; 0.17 \mu \mathrm{M}$ & $3.2 \mu \mathrm{g} ; 0.33 \mu \mathrm{M}$ & $3.3 \mu \mathrm{g} ; 0.36 \mu \mathrm{M}$ \\
\hline
\end{tabular}

Figure 21. An increase of extracellular $\alpha$-synuclein from $0,2 \mu \mathrm{g}$ to $3,3 \mu \mathrm{g}(0,02 \mu \mathrm{M}$ to $0,36 \mu \mathrm{M})$ was observed in cultures overexpressing $\alpha$-synuclein.

(A) Western blot image used to determine the amount (in $\mu \mathrm{g}$ ) and concentration of extracellular and intracellular $\alpha$-synuclein from DIV10 to DIV28. $\alpha$-synuclein was visualized using anti- $\alpha$-synuclein (human-specific, Syn211, ThermoFisher).

(B) Quantification of $\alpha$-synuclein amount from DIV10 to DIV28. $\alpha$-synuclein amount (in $\mu \mathrm{g}$ ) in the lysate or culture medium \pm standard deviation is displayed. Statistical analysis: Two-tailed t-test with Welch's correction (comparison of extracellular to intracellular) or one-way ANOVA (comparison of timepoints), post-hoc statistical power test. $* *$ : $\mathrm{p}<0.01, *$ : $<<0.05$. Statistical power (comparison of extracellular to intracellular): $1-\beta>99,8 \%$ (DIV10), $1-\beta>97,8 \%$ (DIV16), $1-\beta>93,2 \%$ (DIV22), $1-\beta>96,6 \%$ (DIV28). Statistical power (comparison of extracellular synuclein amounts across timepoints): $1-\beta>88,3 \%$ (DIV10 vs DIV22), $1-\beta>96,0 \%$ (DIV10 vs DIV28). Sample size: cell culture lysates/culture media from 4 independent cultures.

(C) Table summarizing the observed amount and concentration of total extracellular and intracellular $\alpha$-synuclein.

Subsequently, an additional experiment was performed that compared the levels of $\alpha$-synuclein in $\alpha$-synuclein overexpressing neurons with the levels of endogenous $\alpha$-synuclein in control neurons. This was done to gather a point of comparison to other studies that had investigated the impact of increased 
levels of $\alpha$-synuclein, such as the study by McDowell et al. which had observed a disruption of rhythmic activity in transgenic rats that expressed 2-5 fold more $\alpha$-synuclein than wild-type rats ${ }^{131,133}$. Thus, to determine the fold increase of $\alpha$-synuclein in $\alpha$-synuclein overexpressing neurons, one population of neurons overexpressed $\alpha$-synuclein, whereas the second population of neurons overexpressed the control protein $\gamma$-synuclein. After determining the amount of $\alpha$-synuclein in these neurons, it was observed that in neurons overexpressing $\alpha$-synuclein, the intracellular level of $\alpha$-synuclein was 18,7 times higher than the endogenous level of $\alpha$-synuclein and that the extracellular level was 23,7 times higher than the endogenous level of $\alpha$-synuclein (Figure 22). This indicated that the expression level was substantially higher in our cultures than in the animals used by McDowell et al. ${ }^{131,133}$.

A

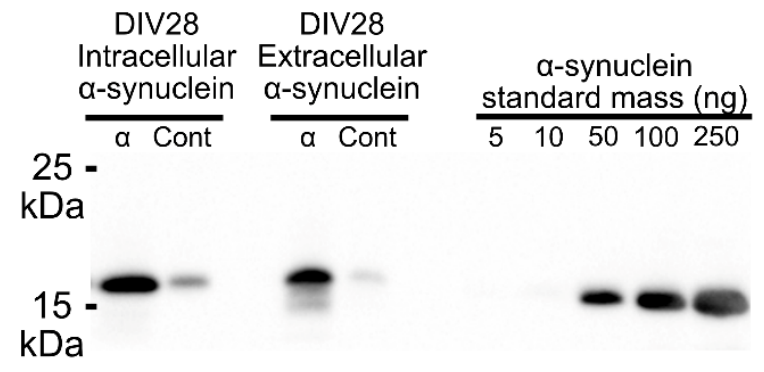

Expressing from DIV4 to DIV28:

$\alpha \quad-\alpha$-synuclein

Cont - Control protein ( $\gamma$-synuclein)
B Intracellular (lysates)

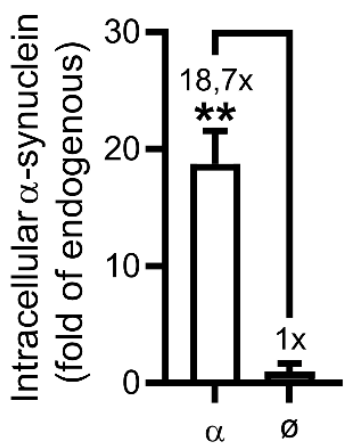

Expressing from DIV4 to DIV28:

$\square$ a-synuclein $\square$ Control protein ( $\gamma$-synuclein)
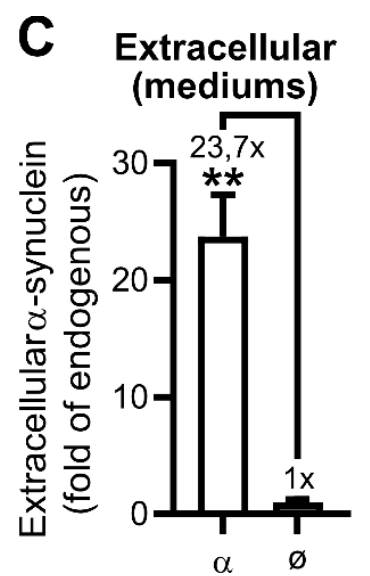

Figure 22. Overexpression of $\alpha$-synuclein increases $\alpha$-synuclein amount in the culture by 18- to 24-fold compared to the level of endogenous $\alpha$-synuclein.

(A) Western blot image for quantifying intracellular and extracellular $\alpha$-synuclein amount in neurons overexpressing $\alpha$-synuclein $(\alpha)$ or control protein $\gamma$-synuclein (Cont/ $\varnothing$ ). $\alpha$-synuclein visualization was performed using anti- $\alpha$-synuclein (BD610787, BD Biosciences) which has the ability to bind human, mouse and rat $\alpha$ synuclein (epitope - rat $\alpha$-synuclein amino acids 15 to 123 ).

(B-C) Quantification of intracellular (B) and extracellular (C) $\alpha$-synuclein amount. Synuclein amount as fold increase of endogenous $\alpha$-synuclein \pm standard deviation is displayed, alongside the numerical value of the fold increase above the error bar. Statistical analysis: Two-tailed t-test with Welch's correction, post-hoc statistical power test. **: $\mathrm{p}<0.01$, statistical power of comparison: $1-\beta>100 \%$. Sample size: cell culture lysates/media from 3 independent cultures.

\subsection{Neuronally produced extracellular $\alpha$-synuclein does not cause the reduction of neuron network bursting}

As our cultures contained $\alpha$-synuclein in the cell culture supernatant, it opened up the possibility that the reduction of network burst frequency was caused by extracellular $\alpha$-synuclein. Indeed, previous studies had already shown that extracellular $\alpha$-synuclein could alter the functioning of cultured neuron networks in a variety of ways, including by reducing the frequency of network bursts 135,136,273.

Notably however, previous studies probed the impact of $\alpha$-synuclein using aggregated recombinant $\alpha$-synuclein, which is a problematic sample due to two reasons. Firstly, the purity of these samples is a known problem, as producing the samples in bacteria leads to the presence of contaminants, including 
lipopolysaccharide endotoxins that change the properties of $\alpha$-synuclein ${ }^{285}$. Secondly, it is not clear whether artificially produced and aggregated recombinant $\alpha$-synuclein is a relevant analogue to the types of $\alpha$-synuclein in patients, as they are produced in bacteria, aggregated artificially and sonicated before application on neurons ${ }^{286}$.

In contrast, the extracellular $\alpha$-synuclein in our study avoided several of these issues. Unlike recombinant $\alpha$-synuclein, the $\alpha$-synuclein in our cultures was free of bacterial contaminants, as it was produced by neurons and not bacteria. Additionally, the $\alpha$-synuclein in our cultures was a more relevant analogue to pathological species of $\alpha$-synuclein in patients because the protein in our study was produced inside mammalian neurons using mammalian protein production machinery.

Importantly, the cell culture supernatant in our cultures contained similar amounts of $\alpha$-synuclein as the amounts that had been shown to trigger an impact on neuron networks in previous studies. More specifically, our cultures contained $0.33-0.36 \mu \mathrm{M}$ extracellular $\alpha$-synuclein, whereas previous studies had used 0,13 or $0,5 \mu \mathrm{M}$ recombinant aggregated $\alpha$-synuclein ${ }^{135,136}$. Therefore, our study was suitable to verify whether extracellular $\alpha$-synuclein really reduced neuron network burst frequency or not.

In order to test this, an assay was designed where the culture medium containing the extracellular species was transferred from donor neurons overexpressing $\alpha$-synuclein to receptor neurons, which did not overexpress $\alpha$-synuclein. More specifically, the first group of neurons was induced to express $\alpha$-synuclein from DIV4 to DIV22. At DIV22, the level of extracellular $\alpha$-synuclein was around 0,33 $\mu \mathrm{M}$ $(3,2 \mu \mathrm{g})$, at which point the medium was transferred to similarly aged receptor neurons. The receptor neurons were imaged before, 1 day, 6 days and 9 days after medium exchange. As a control, untreated neurons that did not receive the $\alpha$-synuclein containing medium were used.

When these medium exchanges were conducted, no significant change of the frequency of network bursting was observed. This was the case 1, 6 and 9 days after the tested neurons received $0,33 \mu \mathrm{M}(3,2$ $\mu \mathrm{g})$ neuronally produced extracellular $\alpha$-synuclein (Figure 23). As mentioned - previous studies had indicated that a treatment with comparable concentrations $(0,13-0,5 \mu \mathrm{M})$ of recombinant $\alpha$-synuclein and for a similar duration ( 1 or 7 days post treatment) produced a reduction in bursting frequency ${ }^{135,136}$. Our results contradicted these observations.

The results thus showed that extracellular $\alpha$-synuclein could not trigger the reduction of burst frequency by itself, but it did not show whether the presence of extracellular $\alpha$-synuclein was necessary to maintain an already present reduction in burst frequency. In order to answer this question, it was therefore tested whether the reduction of burst frequency in $\alpha$-synuclein overexpressing neurons would be rescued when the extracellular $\alpha$-synuclein present in the cell culture supernatant was removed. 
A produce and release $0,33 \mu \mathrm{M} \alpha$-synuclein

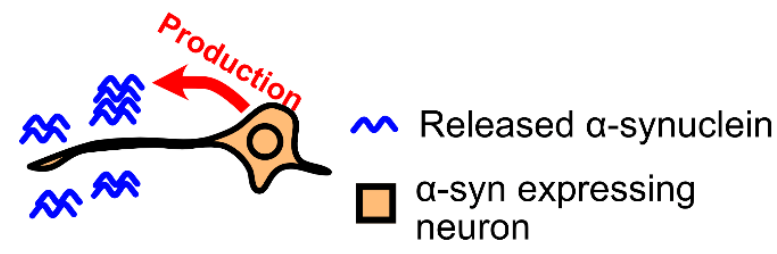

On DIV22, untreated neurons receive medium from the donor neurons with $\alpha$-synuclein

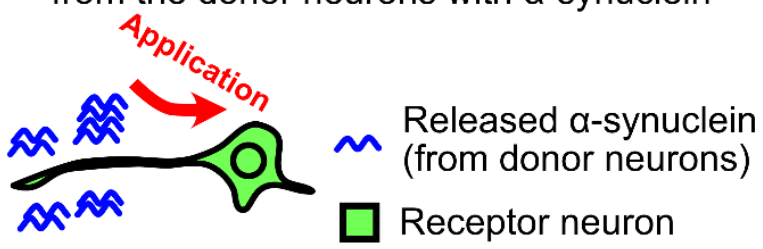

The network activity of receptor neurons was recorded pre-treatment, as well as 1,6 and 9 days post-treatment
B

Impact of medium from donor neurons with extracellular $\alpha$-synuclein

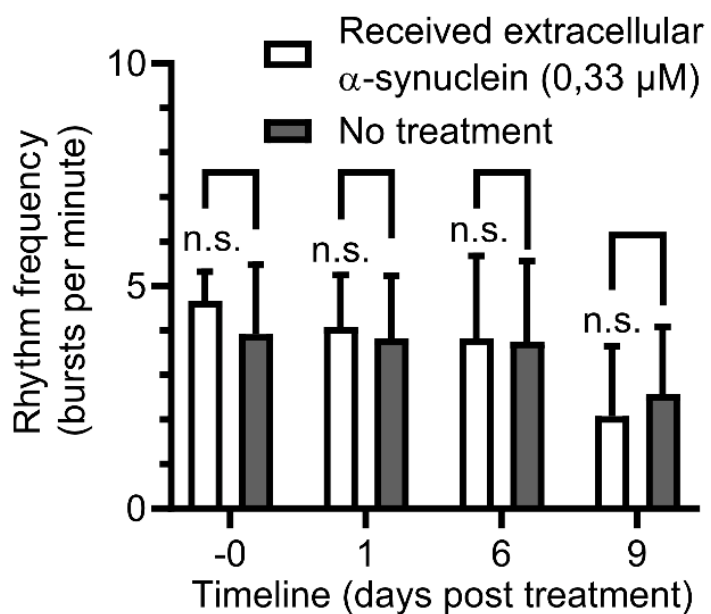

Treatment carried out DIV22

\section{The impact of extracellular $\alpha$-synuclein - summary of studies}

\begin{tabular}{|c|c|c|c|c|}
\hline Study: & $\begin{array}{l}\text { Type of extracellular } \\
\alpha-\text {-synuclein tested: }\end{array}$ & $\begin{array}{c}\text { Concentration of } \\
\text { tested extracellular } \\
\alpha-s y n u c l e i n:\end{array}$ & $\begin{array}{l}\text { Does treatment } \\
\text { reduce bursting } \\
\text { frequency? }\end{array}$ & $\begin{array}{l}\text { Timepoint of first } \\
\text { burst frequency } \\
\text { reduction }\end{array}$ \\
\hline This study & $\begin{array}{l}\alpha \text {-synuclein species } \\
\text { released from } \\
\text { mammalian neurons }\end{array}$ & $\begin{array}{l}0,33 \mu \mathrm{M} \\
\text { (DIV22) }\end{array}$ & No & - \\
\hline Gribaudo et al. & $\begin{array}{l}\text { Artificially aggregated } \\
\text { recombinant } \\
\text { a-synuclein fibrils }\end{array}$ & $0,5 \mu \mathrm{M}$ & Yes & $\begin{array}{l}1 \text { day post treatment } \\
\text { with } \alpha \text {-synuclein }\end{array}$ \\
\hline Froula et al. & $\begin{array}{c}\text { Artificially aggregated } \\
\text { recombinant } \\
\alpha \text {-synuclein fibrils }\end{array}$ & $\begin{array}{l}0,13 \mu \mathrm{M} \\
(2 \mu \mathrm{g} / \mathrm{ml})\end{array}$ & $\begin{array}{c}\text { Yes } \\
\text { (of individual neurons) }\end{array}$ & $\begin{array}{c}7 \text { days post treatment } \\
\text { with } \alpha \text {-synuclein }\end{array}$ \\
\hline
\end{tabular}

Figure 23. Treatment of neurons using culture medium that contains $0,33 \mu \mathrm{M}$ extracelullar $\alpha$-synuclein does not reduce the frequency of network bursting.

(A) Schematic summarizing the experimental concept. Extracellular $\alpha$-synuclein illustrated as blue lines.

(B) Quantification of the frequency of neuron network bursting in the presence of $0,33 \mu \mathrm{M}$ extracellular $\alpha$-synuclein produced by donor neurons. The frequency of network bursts \pm standard deviation is depicted. The timepoints are: pre-treatment ("-0"), 1, 6 and 9 days post treatment. Statistical analysis: Two-tailed t-test with Welch's correction, post-hoc statistical power test. "n.s" = not significant. Sample size: 12 independent cultures. (C) Comparison of results in subfigure $\mathrm{C}$ with previously published results ${ }^{135,136}$.

(A-C) This figure was created in collaboration with Dr. Pretty Garg.

In order to test this, $\alpha$-synuclein was overexpressed in neurons from DIV4 to DIV28, at which point the level of extracellular $\alpha$-synuclein was determined to be $0,36 \mu \mathrm{M}$ (Figure 21). As mentioned, at this timepoint, neurons already exhibited a robust reduction of burst frequency (Figure 18). At DIV28, the medium containing $0,36 \mu \mathrm{M}$ extracellular $\alpha$-synuclein was washed off by removing the culture medium and replacing it with medium from control neurons that had not overexpressed $\alpha$-synuclein. To assess the impact on neuronal network activity, the frequency of network bursts was determined before, 1 day after and 3 days after medium exchange. Doing this however, the reduction of burst frequency induced by $\alpha$-synuclein overexpression was not rescued by washing off extracellular $\alpha$-synuclein (Figure 24 ).

This therefore suggested that the presence of extracellular $\alpha$-synuclein was not required to maintain the reduction of burst frequency. 
A a-synuclein expressing neurons produce and release $0,36 \mu \mathrm{M} \alpha$-synuclein

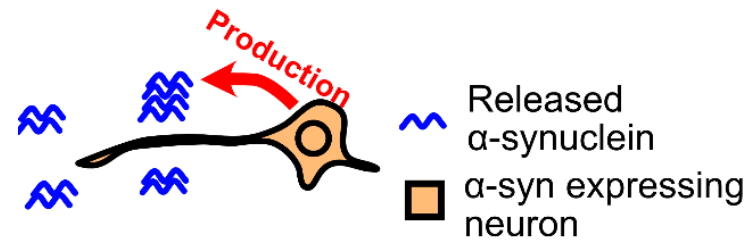

On DIV28, the extracellular $\alpha$-synuclein was washed off by replacing the medium

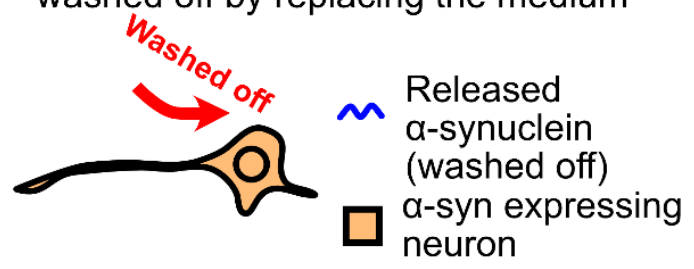

The receptor neurons were imaged pre-treatment, as well as 1 and 3 days post-treatment

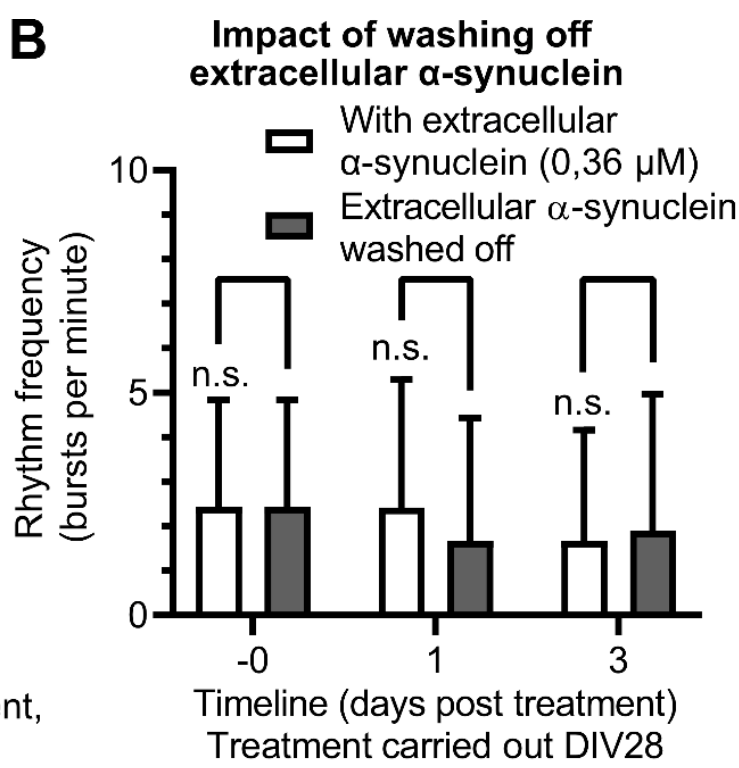

Figure 24. Washing off extracellular $\alpha$-synuclein does not rescue the reduction of neuron network burst frequency by $\alpha$-synuclein overexpression.

(A) Schematic summarizing experimental concept. Extracellular $\alpha$-synuclein illustrated as blue lines.

(B) Quantification of the impact of washing off the extracellular $\alpha$-synuclein on neuron network burst frequency. The frequency of network bursts \pm standard deviation is depicted. The timepoints are: pre-treatment $(-0), 1$ and 3 days post treatment. Statistical analysis: Two-tailed t-test with Welch's correction, post-hoc statistical power test. "n.s" = not significant. Sample size: 12 independent cultures.

(A-C) This figure was created in collaboration with Dr. Pretty Garg.

\section{5. $\alpha$-synuclein overexpression reduces the number of active pre-synapses and axons}

The previous experiments indicated that overexpression of $\alpha$-synuclein reduced the frequency of network bursting and that this reduction did not depend on extracellular $\alpha$-synuclein. Figure 20 shows that the number of neurons participating in network activity was also reduced, which was taken as a possible indicator of diminished neuronal connectivity. Thus, reduction of connectivity by $\alpha$-synuclein overexpression was considered a promising topic of investigation. In line with this consideration, a variety of studies suggested that connectivity between cells is a key parameter impacting on rhythmic activity, including studies using computer modelling to describe the behavior of neuronal networks ${ }^{16-20}$.

Therefore, in the next experiment it was investigated whether $\alpha$-synuclein overabundance caused lesions on structures important for keeping the neuronal network in a functional state of connectivity, such as pre-synapses and neurites. For characterizing pre-synapses, the fluorescent sensor synaptophysinGCaMP was used, as it allowed for unambiguous discrimination of active synapses from inactive synapses and debris - only active synapses take up calcium and thus have a dynamic signal (Figure 5). Dynamic imaging provided an advantage over the use of more static detection methods like immunocytochemistry, where separating debris from active synapses would be difficult. Indeed, our initial attempts of synaptic quantification using a synapsin stain revealed a very densely stained neurite 
network, where reliable separation of synapses and debris was determined to be complicated (Supplementary Figure 1).

A

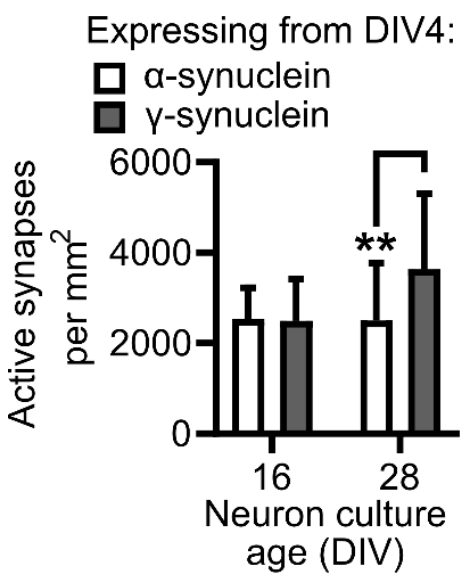

D

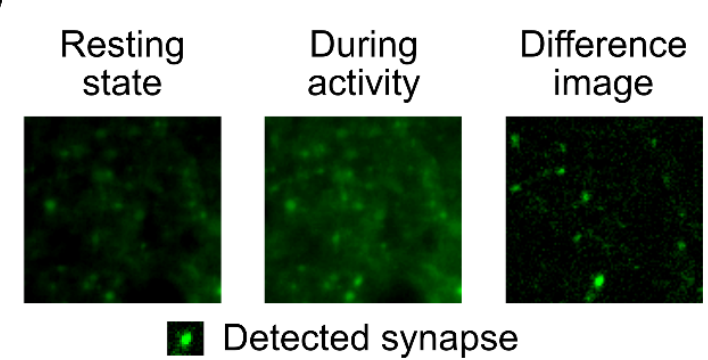

B

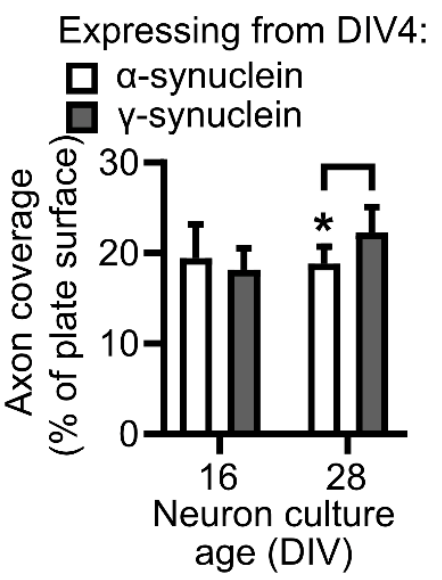

E

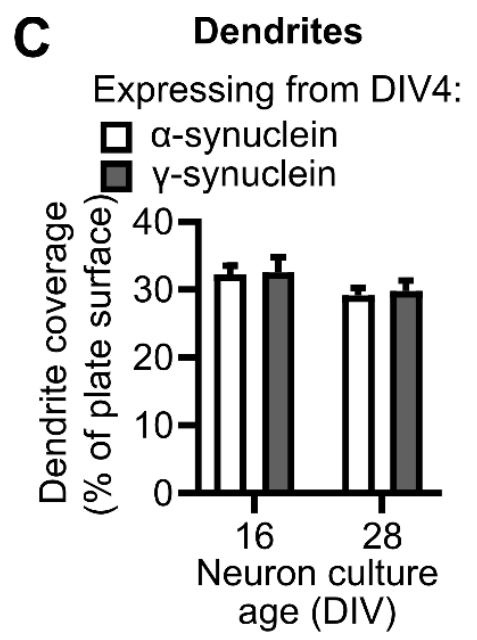

$\mathbf{F}$

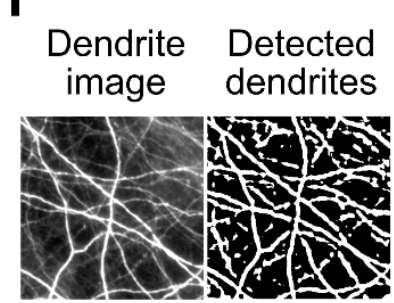

$\square$ Detected areas

Figure 25. $\alpha$-synuclein overexpressing neurons have fewer active pre-synapses and a lower axon coverage. (A-C) Quantification of the amount of active pre-synapses (A), axons (B) and dendrites (C). The figure displays the amount of pre-synapses per $\mu \mathrm{m}^{2} \pm$ standard deviation $(\mathbf{A})$ or the amount of well surface covered by neurites \pm standard deviation (B-C). Comparison between: $\alpha$-synuclein (white bars) and neurons overexpressing $\gamma$-synuclein (gray bars). Statistical analysis: two-tailed t-test with Welch's correction and a post-hoc power test. ****: 0.0001, $*_{*}: \mathrm{p}<0.01, *: \mathrm{p}<0.05$. Statistical power: $1-\beta>81 \%$ (A), $1-\beta>75,9 \%$ (B), sample size of independent cultures at DIV28: 28 (A), 8 (B, C). At DIV15: 9 (A), 6 (B), 4 (C).

(D) Pre-synapse detection images. Image of neurons overexpressing synaptophysin-GCaMP during resting state (left image) and activity (middle image). Calculated difference between left and center image, with intensity in the image corresponding to the degree of difference (right image).

(E-F) Neurite coverage detection. Representative images (left panels) of either axons stained for neurofilament L (E) or dendrites stained for MAP2 (F) alongside an image of neurites detected using a computer analysis (right panels).

For the characterization and quantification of neuritic structures, the axons and dendrites were analyzed separately by making use of immunocytochemical staining using axon and dendrite specific markers with neurofilament $\mathrm{L}$ being used as an axonal marker and MAP2 as a dendritic marker ${ }^{253,269,270}$. To prove that these markers were indeed specific for axons and dendrites, a co-stain was performed, showing that the markers were located in distinctive structures (Figure 13). For computational analysis of the neurites, an ImageJ-based computer algorithm was used (Figure 14).

After establishing the imaging methods, the next step was to determine whether $\alpha$-synuclein impacted on the number of active pre-synapses, axons and dendrites on DIV16 and DIV28. DIV16 was chosen because on this timepoint, $\alpha$-synuclein did not yet impact on neuronal network activity, whereas DIV28 was chosen because $\alpha$-synuclein demonstrated a full-blown reduction in neuronal bursting. Indeed, 
$\alpha$-synuclein overexpression caused a significant reduction in both number of active pre-synapses and amount of axons on DIV28 when compared to the overexpression of $\gamma$-synuclein, whereas no reduction was observed on DIV15. In the case of pre-synapses at DIV28, a $28 \%$ reduction was observed, whereas for axons, a $15 \%$ reduction was observed. In the case of dendrites, no reduction of the number of active synapses or amount of axons was observed at DIV16 (Figure 25). These results thus demonstrated that $\alpha$-synuclein overabundance resulted in a concomitant loss of axonal and pre-synaptic integrity, providing a reasonable explanation for the proposed loss of network connectivity.

\section{6. $\alpha$-synuclein overexpression reduces the level of cytosolic cyclic AMP}

Results obtained so far demonstrated that $\alpha$-synuclein overabundance significantly diminished structurally important network connectors, i.e. axons and especially the number of active presynapses. However, it was also determined that a reduction in axons and pre-synapses was unlikely to be a full explanation for the reduction of neuron network burst frequency by $\alpha$-synuclein overexpression. This was based on two reasons. Firstly, reduction of axons (-15\%) and pre-synapses (-28\%) at DIV28 was smaller in effect size than the reduction in burst frequency at DIV28 (-56\%) (Figure 18, Figure 25). This could indicate that additional factors were involved. Secondly, there was no reduction of axons and presynapses observed at DIV15, despite a small significant reduction of burst frequency at this timepoint. As a result, the investigation was continued, with the hope of finding other neuronal physiology impairments induced by $\alpha$-synuclein overexpression.

Beyond the structural integrity of axons and synapses, a variety of mechanisms are known to impact neuronal connectivity. Among these is synaptic potentiation, which is a phenomenon where repeated activity causes the strengthening of synapses ${ }^{4}$. This in turn is regulated by several pathways, one of which involves the signaling molecule cyclic AMP, which was a promising target for our investigation due to three reasons ${ }^{4,212}$. Firstly, it could impact neuronal connectivity not only by regulating synaptic potentiation but also by activating HCN channels, which are known to regulate the cellular excitability of neurons. Secondly, various studies had shown that in the case of synucleinopathies, the activity of the cAMP-producing enzyme adenylyl cyclase is altered ${ }^{34-36,240,241}$. Thirdly, any results gathered on cAMP in the context of $\alpha$-synuclein would be novel, as previous studies had not addressed the connection between $\alpha$-synuclein and cAMP.

Before this could be done however, it was necessary to show that neuron network bursting was impacted by the two downstream targets of cAMP, synaptic potentiation and $\mathrm{HCN}$ channel functioning. To determine this, cultures prepared using the standard experimental setup were treated on DIV28 with either an activator of synaptic potentiation (BDNF) or an inhibitor of HCN channels (ZD-7288). Doing this, it was observed that application of BDNF significantly increased neuron network burst frequency, whereas application of ZD-7288 significantly reduced neuron network burst frequency (Figure 26). This showed that neuron network bursting was indeed impacted by pathways regulated by cAMP. 


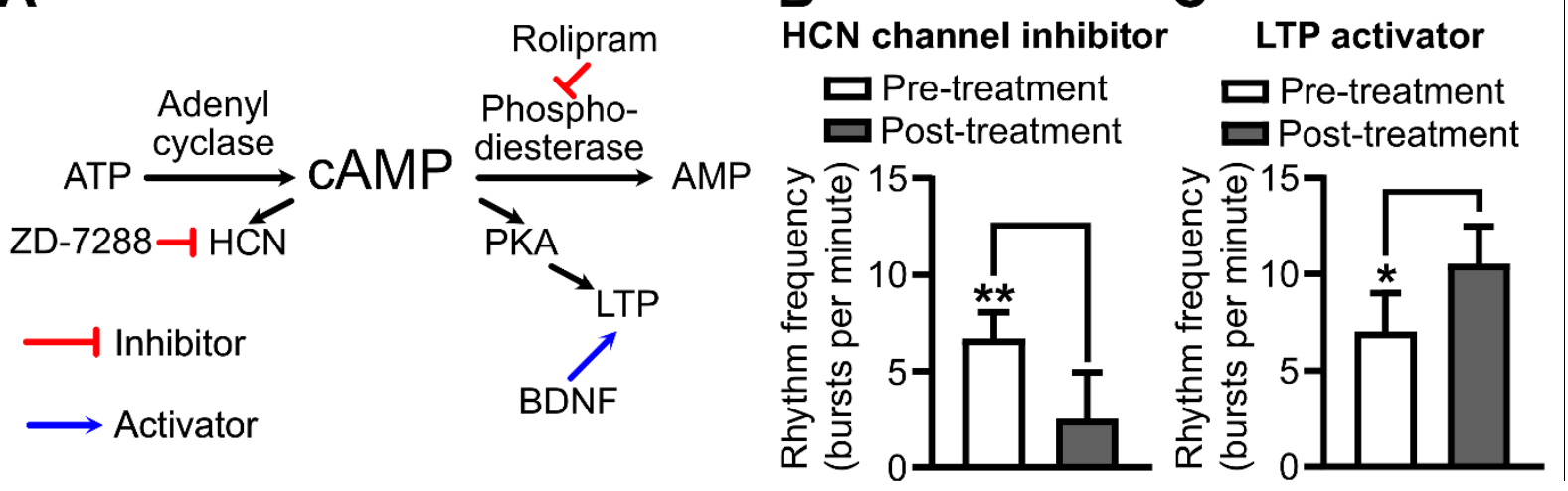

Figure 26. HCN channels and synaptic potentiation, which are downstream targets of cAMP signaling, regulate neuron network bursting frequency inside cultured neurons.

(A) Illustration of cyclic AMP metabolism. cAMP = cyclic AMP, inhibitors = red T-shaped lines, activators = blue arrows, ATP $=$ adenosine triphosphate, $\mathrm{AMP}=$ adenosine monophosphate, $\mathrm{HCN}=$ hyperpolarization-activated cyclic nucleotide gated channels, $\mathrm{BDNF}=$ brain-derived neurotrophic factor, $\mathrm{PKA}=$ protein kinase $\mathrm{A}, \mathrm{LTP}=\mathrm{long}$ term potentiation,

(B) Quantification of neuronal network burst frequency at DIV 28 before (white bar) and 30 min after (grey bar) application of the HCN channel inhibitor ZD-7288 $(10 \mu \mathrm{M})$.

(C) Quantification of neuronal network burst frequency at DIV 28 before (white bar) and 30 min after (grey bar) application of BDNF (2 nM).

(B-C) Statistical analysis by two-tailed t-test with Welch's correction and a post-hoc power test. **: p < 0.01, *: p $<0.05$. Statistical power $(1-\beta$ error probability $)=90.9 \%$ for $(\mathbf{B})$ and $78.4 \%$ for $(\mathbf{C})$. $N=6$ independent neuron cultures for each time point (B-C).

After this, it was analyzed whether adding a compound that increases cellular cyclic AMP levels would change the frequency of neuron network bursting. This test was carried out using rolipram, which inhibits the cAMP-degrading enzyme phosphodiesterase. Indeed, when $\gamma$-synuclein overexpressing neurons were treated with rolipram at DIV28, the frequency of network bursting significantly increased (Figure 27A). In contrast, when $\alpha$-synuclein overexpressing neurons were subjected to an identical treatment with rolipram, no increase of neuron network bursting was observed (Figure 27A).

This result therefore indicated a possible reduction of cAMP levels induced by $\alpha$-synuclein overexpression. However, this needed to be investigated further by determining whether $\alpha$-synuclein overexpression changed the levels of cytosolic cAMP. For this, two sensors were used. The first sensor that was selected was cAMPr, which is a GFP-based sensor that directly measures cAMP (Figure 6). The second sensor selected was AKAR4-cAMPs, which is a FRET sensor that measures the activity of PKA, a type of kinase directly regulated by levels of cAMP (Figure 7). Two sensors were used so they could be used as an internal control for each other.

It was observed that when using the cAMPr sensor, neurons overexpressing $\alpha$-synuclein exhibited a statistically significant $6 \%$ reduction of cAMPr fluorescence compared to neurons overexpressing $\gamma$-synuclein on DIV28 (Figure 27B). In agreement with this finding, a statistically significant 6\% reduction of the PKA phosphorylation rate was observed on DIV28 in neurons overexpressing $\alpha$-synuclein compared to neurons overexpressing $\gamma$-synuclein when using the AKAR4-cAMPs sensor (Figure 27C). No differences were found using either sensor on DIV15. As both sensors demonstrated a reduction identical in effect size, these results supported the claim that $\alpha$-synuclein reduced the level 
of cAMP compared to the overexpression of $\gamma$-synuclein (Figure 27). The observed reduction in cAMP levels on DIV28 was thus an additional explanation for the robust reduction of neuron network burst frequency by $\alpha$-synuclein overexpression on DIV 28 .

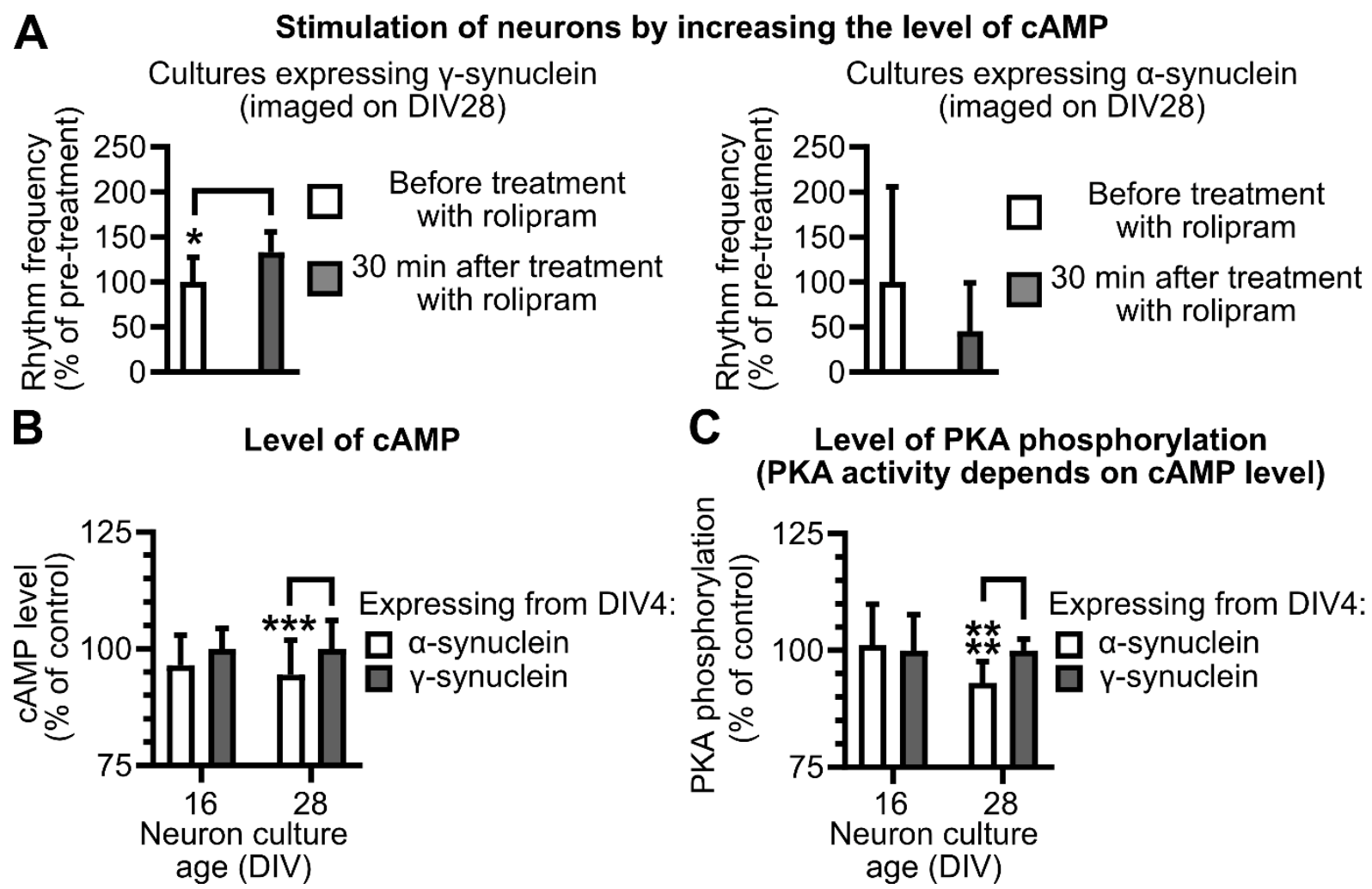

Figure 27. $\alpha$-synuclein overexpression reduces the ability of calcium bursting to be activated by molecules increasing the level of cAMP and reduces cytosolic levels of cAMP.

(A) Quantification of the frequency of network bursting before (white bars) and after (gray bars) treatment with a cAMP level increasing molecule ( $1 \mu \mathrm{M}$ rolipram) alongside $\alpha$-synuclein overexpression (left panel) or $\gamma$-synuclein overexpression (right panel). Network burst frequency as $\%$ of control \pm standard deviation is indicated. Statistical analysis (used for all subfigures): two-tailed t-test with Welch's correction and a post-hoc power test. *: $\mathrm{p}<0.05$, $* * *: \mathrm{p}<0.0001, * * * * \mathrm{p}<0.0001$. Statistical power: $1-\beta>54.5 \%$. Sample size: 6 independent cultures.

(B-C) Quantification of the levels of cAMP (B) or cAMP-regulated PKA phosphorylation (C). The level of cAMP as $\%$ of control \pm standard deviation (B) or the level of PKA phosphorylation as $\%$ of control \pm standard deviation is depicted (C). The sensors cAMPr (B) and AKAR4-cAMPs (C) were used. Comparison between: neurons overexpressing $\alpha$-synuclein (white bars) or $\gamma$-synuclein (gray bars). Statistical power: $1-\beta>93.2 \%$ (B), $1-\beta>$ 100\% (C). Sample size of independent cultures: 6 for DIV15, 36 for DIV28 (B), 12 for DIV15, 18 for DIV28 (C).

\subsection{The $\beta$-synuclein P123H mutant reduces the frequency of network bursts, whereas wild-type $\beta$-synuclein and the $\beta$-synuclein V70M mutant do not}

Subsequently, it was investigated whether the reduction of burst frequency was unique to $\alpha$-synuclein overexpression or whether it could also be triggered by the overexpression of other synuclein variants variants such as wild-type $\beta$-synuclein, the $\beta$-synuclein $\mathrm{P} 123 \mathrm{H}$ mutant and the $\beta$-synuclein V70M mutant ${ }^{78,83,84}$. For this, an identical experimental setup was used as detailed earlier for $\alpha$-synuclein, i.e. including co-expression of $\mathrm{Bcl}-\mathrm{xL}$ in order to prevent loss of neurons from the network. It was thus observed that wild-type $\beta$-synuclein overexpression did not significantly reduce the frequency of network bursting compared to overexpression of wild-type $\gamma$-synuclein, except for a small reduction at DIV15. In contrast, 
it was found that neurons overexpressing the $\beta$-synuclein $\mathrm{P} 123 \mathrm{H}$ mutant reduced the frequency of network bursting by $28-56 \%$ between DIV16 and DIV31 in comparison to neurons overexpressing $\gamma$ synuclein. Finally, it was observed that the $\beta$-synuclein V70 mutant did not significantly reduce burst frequency compared to neurons overexpressing $\gamma$-synuclein (Figure 28).
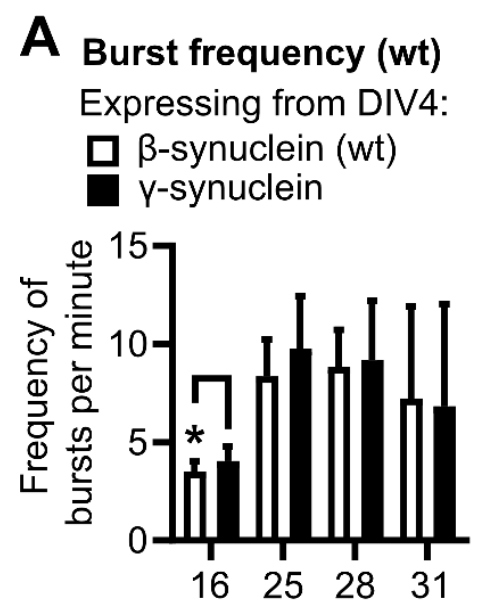

Neuron culture age (DIV)
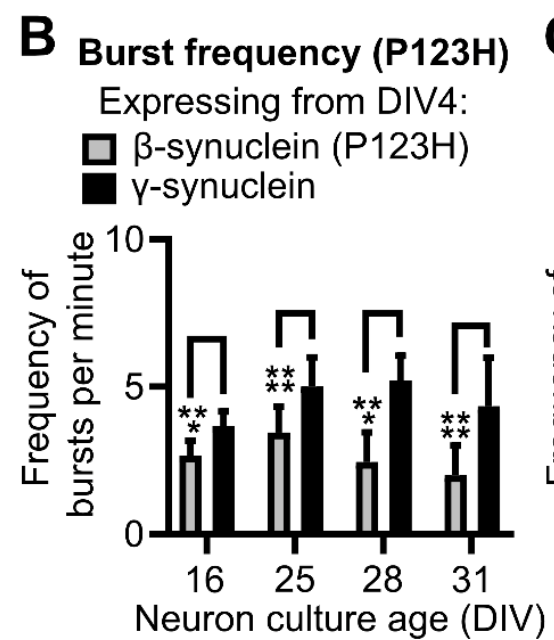
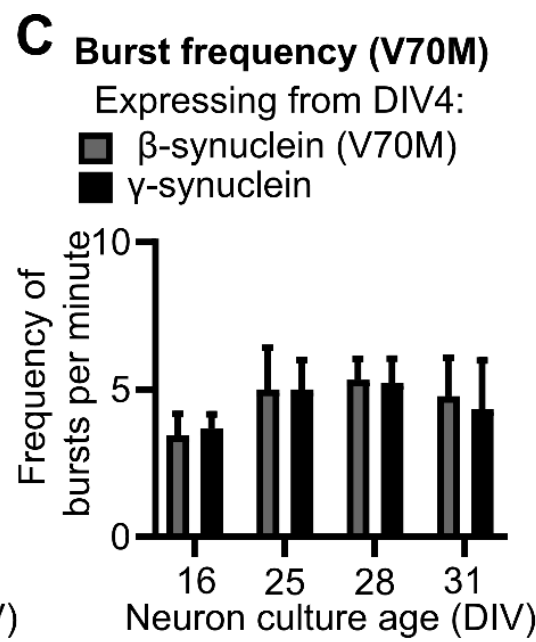

Figure 28. The P123H $\beta$-synuclein mutant reduces the frequency of network bursting at four different timepoints, whereas the V70M mutant and wild-type $\beta$-synuclein induce no consistent reduction of burst frequency.

(A-C) Quantification of network burst frequency in wild-type $\beta$-synuclein overexpressing neurons (white bars) (A), P123H $\beta$-synuclein mutant overexpressing neurons (light gray bars) (B) or V70M $\beta$-synuclein mutant overexpressing neurons (dark gray bars) (C), with all three compared to $\gamma$-synuclein overexpressing neurons (black bars). The figure indicates the average number of network bursts per minute \pm standard deviation. $\mathrm{wt}=\beta$-synuclein (wt), $\mathrm{P} 123 \mathrm{H}=\mathrm{P} 123 \mathrm{H} \beta$-synuclein mutant, V70M = V70M $\beta$-synuclein mutant. Statistical analysis (used for all subfigures): two-tailed t-test with Welch's correction and a post-hoc power test. *: p < 0.05 . $* * *$ : p < 0.001 , $* * * *: p<0.0001$. Statistical power: $1-\beta>73.1 \%$ (DIV16) (A), $1-\beta>100 \%$ (DIV16, DIV25, DIV28, DIV32) (B). Sample size of independent cultures: 18 (A), 9 (B-C).

This result was contrary to expectations, as previous studies had suggested that all of the $\beta$-synuclein variants were neurotoxic in terms of causing neuronal cell death ${ }^{78,84}$. To determine whether these mutants still caused cell death, neurons were induced to overexpress either one of the $\beta$-synuclein variants or $\gamma$-synuclein from DIV2. Notably, overexpression of Bcl-xL was omitted, as it inhibits cell death and thus does not permit the assessment of cell survival. It was thus observed that of the three variants only the $\beta$-synuclein $\mathrm{P} 123 \mathrm{H}$ mutant moderately but significantly reduced cell survival when it was overexpressed from DIV4 to DIV28. In contrast, the overexpression of the V70M $\beta$-synuclein mutant or of wild-type $\beta$-synuclein did not reduce cell survival (Figure 29). The overexpression of the $\mathrm{P} 123 \mathrm{H} \beta$-synuclein mutant was therefore neurotoxic in terms of its impact on network bursting and cell survival, whereas the overexpression of the other $\beta$-synuclein variants was not.

As the overexpression of the $\beta$-synuclein $\mathrm{P} 123 \mathrm{H}$ mutant was neurotoxic when overexpressed without $\mathrm{Bcl}-\mathrm{xL}$, it was determined whether it was neurotoxic in the presence of $\mathrm{Bcl}-\mathrm{xL}$. This experiment was performed because if $\beta$-synuclein $\mathrm{P} 123 \mathrm{H}$ mutant overexpression induced cell death in the presence of $\mathrm{Bcl}-\mathrm{xL}$, then this would be one explanation as to why overexpression of this mutant reduced the frequency of network bursting (which was recorded in cultures expressing Bcl-xL). However, 
overexpression of the $\beta$-synuclein $\mathrm{P} 123 \mathrm{H}$ mutant did not increase levels of cell death in the presence of Bcl-xL overexpression (Figure 30).

A

Cell survival (wt)

Expressing from DIV4:

을-synuclein (wt)

y-synuclein

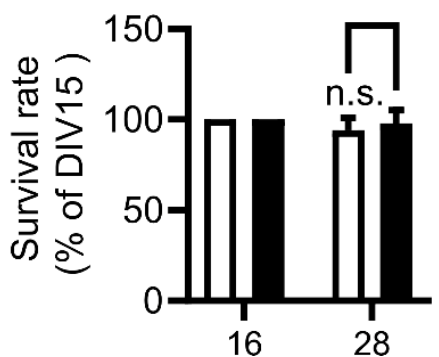

B Cell survival (P123H)

Expressing from DIV4:

口 $\beta$-synuclein (P123H)

Y-synuclein

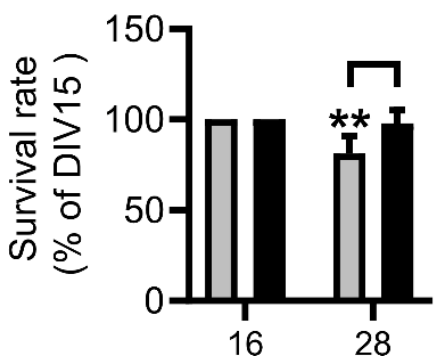

C Cell survival (V70M)

Expressing from DIV4:

$\square$ B-synuclein (V70M)

y-synuclein

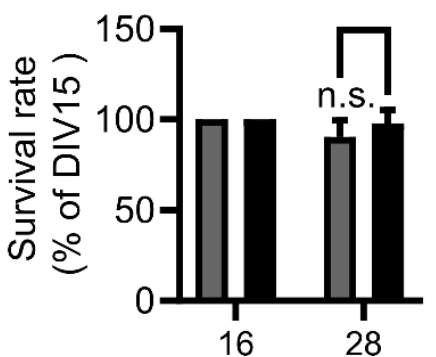

Figure 29. Overexpression of the P123H $\beta$-synuclein mutant reduces the survival rate of cells at DIV28, whereas the overexpression of the V70M $\beta$-synuclein mutant or of wild-type $\beta$-synuclein does not.

(A-C) Quantification of cell survival in wild-type $\beta$-synuclein overexpressing neurons (white bars) (A), P123H $\beta$-synuclein mutant overexpressing neurons (light gray bars) (B) or V70M $\beta$-synuclein mutant overexpressing neurons (dark gray bars) (C), with all three compared to $\gamma$-synuclein overexpressing neurons (black bars). Antiapoptotic factor Bcl-xL was not expressed in these cultures. The figure indicates the average percentage of surviving cells from DIV16 to DIV28 as \% of the number of cells on DIV15. "wt" $=\beta$-synuclein (wt), P123H = $\mathrm{P} 123 \mathrm{H} \beta$-synuclein mutant, $\mathrm{V} 70 \mathrm{M}=\mathrm{V} 70 \mathrm{M} \beta$-synuclein mutant. Statistical analysis (used for all subfigures): two-tailed t-test with Welch's correction and a post-hoc power test. "n.s." = not significant $* *: p<0.01$. Statistical power: $1-\beta>97.8 \%(\mathbf{B})$, Sample size of independent cultures: 9 (A-C).

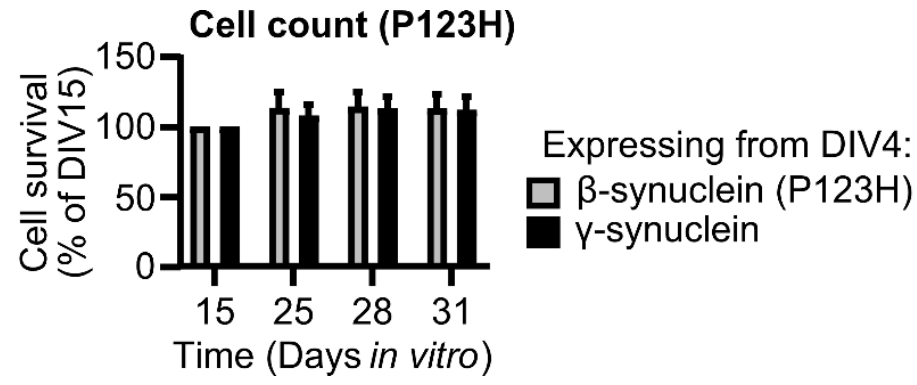

Figure 30. Overexpression of the P123H $\beta$-synuclein mutant is not toxic to neurons when overexpressed alongside Bcl-xL.

Quantification of cell survival in neurons overexpressing P123H $\beta$-synuclein (light gray bars) compared to neurons overexpressing $\gamma$-synuclein (black bars) in the presence of $\mathrm{Bcl}$-xL overexpression. The figure indicates the average percentage of surviving cells from DIV16 to DIV28 as \% of the number of cells on DIV15. P123H $=\beta$-synuclein P123H mutant. Statistical analysis: two-tailed t-test with Welch's correction and a post-hoc power test. Sample size of independent cultures: 9 .

As cell death was not present in $\beta$-synuclein $\mathrm{P} 123 \mathrm{H}$ mutant overexpressing neurons (when also expressing Bcl-xL), other explanations for the frequency reduction were investigated. Specifically, it was investigated whether overexpressing the $\beta$-synuclein $\mathrm{P} 123 \mathrm{H}$ mutant destroyed neurites or synapses or reduced cellular levels of cAMP, as was observed in $\alpha$-synuclein overexpressing neurons. To provide a point of comparison, the impact of wild-type $\beta$-synuclein overexpression on these parameters was determined as well. It was thus observed that when compared to the overexpression of $\gamma$-synuclein, the overexpression of the $\mathrm{P} 123 \mathrm{H} \beta$-synuclein mutant from DIV4 to DIV28 reduced the amount of axons by $49 \%$ and the amount of dendrites by $8.3 \%$. A non-significant reduction of pre-synapses by $18 \%$ was also 
observed. Finally, a reduction in the level of cAMP-activated PKA phosphorylation was observed by $5 \%$, whereas no reduction of any kind was observed when comparing the overexpression of wild-type $\beta$-synuclein to the overexpression of $\gamma$-synuclein (Figure 31 ).

A

cAMP-activated PKA phosph. level Expressing from DIV4 to DIV28:

口 $\beta$-synuclein (wt) Y-synuclein

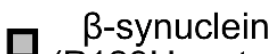
(P123H mutant)
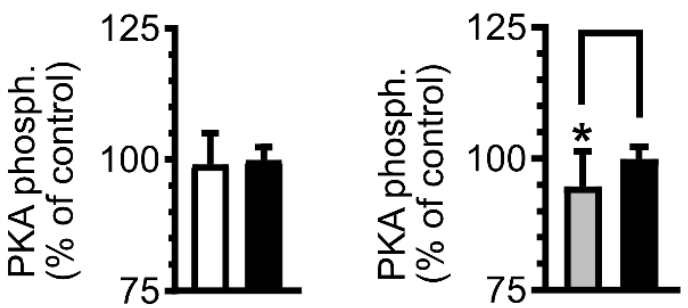

C

$$
\begin{aligned}
& \text { Expressing from DIV4 to DIV28: } \\
& \square \begin{array}{l}
\beta \text {-synuclein }(\mathrm{wt}) \\
\mathrm{y} \text {-synuclein }
\end{array}{ }_{(\mathrm{P} 123 \mathrm{H} \text { m }}^{\beta}
\end{aligned}
$$

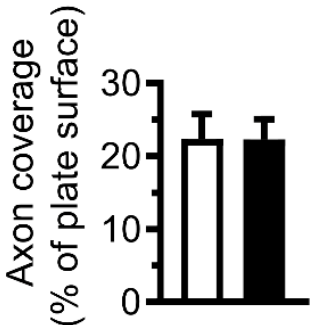

B
Presynapses

Expressing from DIV4 to DIV28:

$\square \beta$-synuclein (wt) $\beta$-synuclein

$\gamma$-synuclein

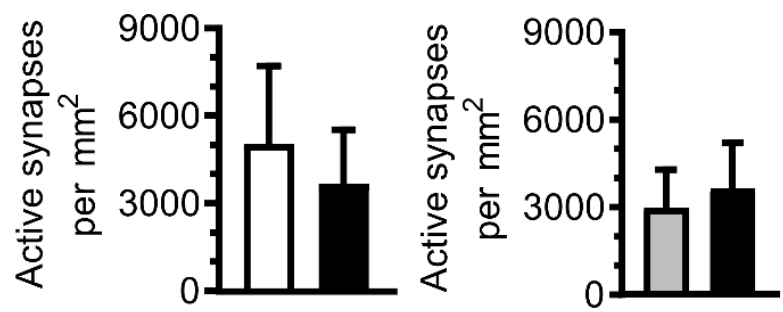

D Dendrites

Expressing from DIV4 to DIV28:

$\square \begin{aligned} & \beta \text {-synuclein (wt) } \\ & \text { y-synuclein }\end{aligned}{ }_{(\mathrm{P} 123 \mathrm{H} \text { mutant })}^{\beta \text {-synuclein }}$
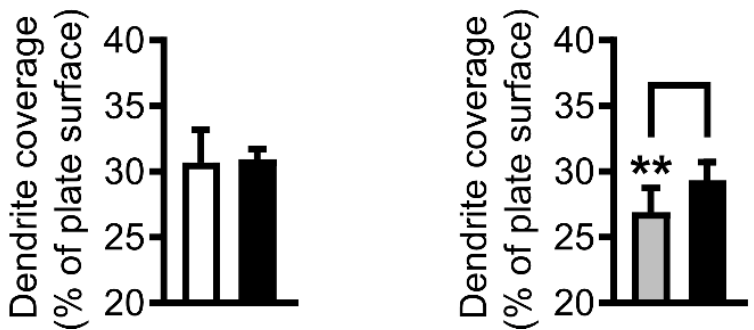

Figure 31. Overexpression of the P123H $\beta$-synuclein mutant reduces the level of cAMP-activated PKA phosphorylation and the amount of neurites, whereas overexpression of wild-type $\beta$-synuclein does not.

(A-D) Quantification of the level of cAMP-regulated PKA phosphorylation (A), amount of pre-synapses (B), axons (C) and dendrites (D) in the presence of either wild-type $\beta$-synuclein overexpression (white bars), mutant P123H $\beta$-synuclein overexpression (light gray bars) or $\gamma$-synuclein overexpression (black bars). The level of PKA phosphorylation as \% of control \pm standard deviation is depicted in (A), the amount of pre-synapses per $\mathrm{mm}^{2} \pm$ standard deviation is depicted in (B), the percentage of plate surface covered by neurites \pm standard deviation is depicted in $(\mathbf{C}, \mathbf{D})$. cAMP = cyclic adenosine monophosphate, $\mathrm{PKA}=$ protein kinase A. Statistical analysis (used for all subfigures): two-tailed t-test with Welch's correction and a post-hoc power test. *: $\mathrm{p}<0.05$. **: $\mathrm{p}<0.01$, ****: $\mathrm{p}<0.0001$. Statistical power: $1-\beta>56.4 \%$ (A), $1-\beta>100 \%$ (C), $1-\beta>91.4 \%$ (D). Sample size of independent cultures: 18 for the left panel and 9 for the right panel (A), 9 for the left panel and 19 for the right panel (B), 8 for the left panel and 10 for the right panel (C), 4 for the left panel and 11 for the right panel (D).

Overall, the overexpression of the $\beta$-synuclein $\mathrm{P} 123 \mathrm{H}$ mutant caused lesions in several structures related to intercellular connectivity, as was observed in neurons overexpressing $\alpha$-synuclein. This therefore supported the case that a reduction in connectivity was the explanation for the reduction of burst frequency in neurons overexpressing the $\mathrm{P} 123 \mathrm{H} \beta$-synuclein mutant.

\subsection{Tau protein reduces the frequency of network bursts, reduces the level of cAMP and the amount of axons, synapses and dendrites}

Next, it was analyzed whether overexpression of the AD-associated tau protein reduced the frequency of network bursting, which was done to determine whether disease-related proteins outside of the 
synuclein family could also impact network bursting in culture ${ }^{5,287}$. Tau protein was a particularly interesting target as studies had shown that it could disrupt rhythmic activity in vivo, which was a similar observation as had been made for $\alpha$-synuclein ${ }^{13-15}$. More specifically, studies had observed that increased CSF levels of tau protein were correlated with disruptions of rhythmic activity in AD patients 14,15

For determining whether tau protein impacted the frequency of rhythmic activity, a similar experimental setup was used as was implemented for the synucleins. However, instead of using one control for tau protein overexpression, two controls were used, with comparisons carried out against $\gamma$-synuclein and NLS-mCherry overexpression. It was observed that compared to overexpression of $\gamma$-synuclein, overexpression of tau protein significantly reduced the frequency of network bursting on DIV16 and from DIV22 to DIV31 (Figure 32). The smallest observed reduction was 18\% at DIV16, whereas the largest observed reduction was 79\% at DIV31. A reduction in the frequency of network bursting was also observed when the overexpression of tau protein was compared to the expression of the secondary negative control, NLS-mCherry (Supplementary Figure 2).

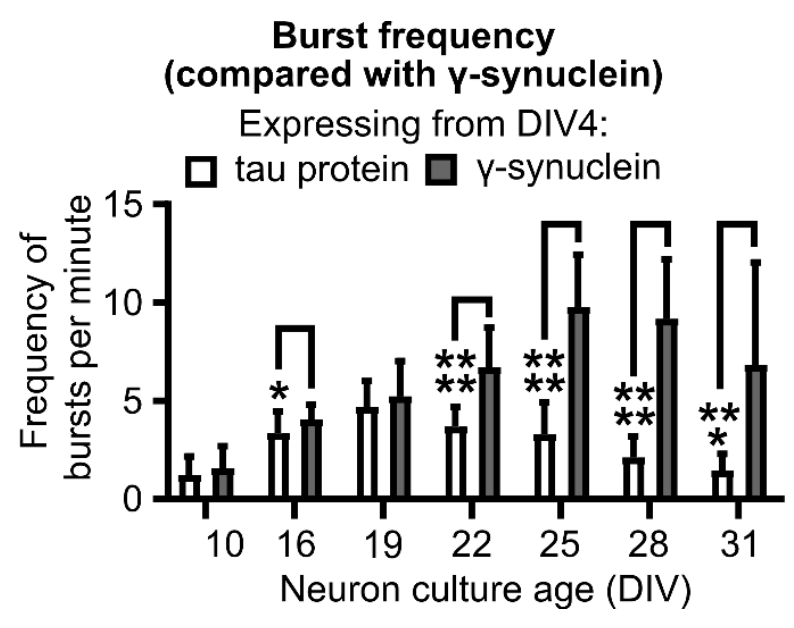

Figure 32. Tau protein overexpression reduces the frequency of calcium bursting.

Quantification of the frequency of calcium bursting in neurons overexpressing tau protein (white bars) compared to neurons overexpressing $\gamma$-synuclein (gray bars). The frequency of bursts per minute \pm standard deviation is depicted. $*: \mathrm{p}<0.05, * * *: \mathrm{p}<0.001, * * * *: \mathrm{p}<0.0001$. Statistical analysis (used for all subfigures): two-tailed t-test with Welch's correction and a post-hoc power test. Statistical power: $1-\beta>75.5 \%$ (DIV16), $1-\beta>100 \%$ (DIV22, DIV25, DIV28), $1-\beta>98.8 \%$ (DIV31). Sample size: 18 independent cultures.

After observing that tau protein reduced the frequency of neuron network bursting, it was further determined whether tau protein overexpression reduced the amount of synapses, neurites or levels of cAMP-activated PKA phosphorylation. This was done to provide an explanation for the reduction of burst frequency by tau protein overexpression. Indeed, tau protein overexpression reduced the amount of pre-synapses by $53 \%$, the amount of axons by $56 \%$, the amount of dendrites by $19 \%$ and the level of cAMP-dependent PKA phosphorylation by $15 \%$ when compared to neurons overexpressing $\gamma$-synuclein. All of the reductions were calculated to be statistically significant (Figure 33). Importantly, similar reductions were observed when the comparison was carried out against NLS-mCherry (Supplementary Figure 3). 
Finally, it was also determined whether tau protein overexpression decreased cell survival, which was done to assess whether the loss of neurites and synapses was a consequence of increased levels of cell death. Furthermore, the impact of tau protein on both burst frequency and on the number of connecting structures was the largest of all the proteins that had been tested, which suggested that it could also be neurotoxic in terms of causing cell death, even when Bcl-xL was overexpressed.

A Presynapses

Expressing from DIV4: tau protein r-synuclein

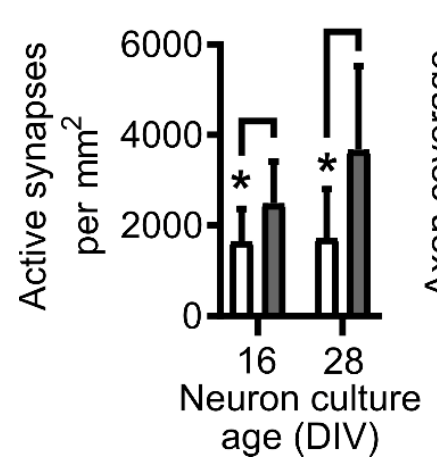

B

Axons

Expressing from DIV4:

$\square$ tau protein

y-synuclein

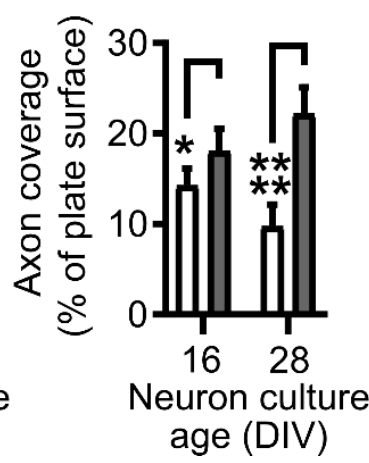

C

Dendrites
D Level of PKA phosphorylation

Expressing from DIV4: Expressing from DIV4:

$\square$ tau protein

y-synuclein

tau protein

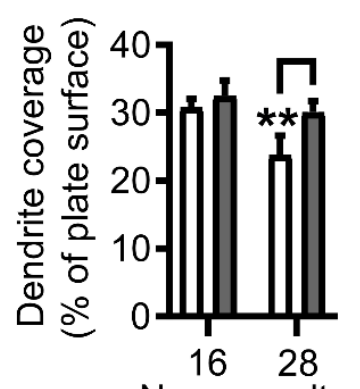

Neuron culture age (DIV)

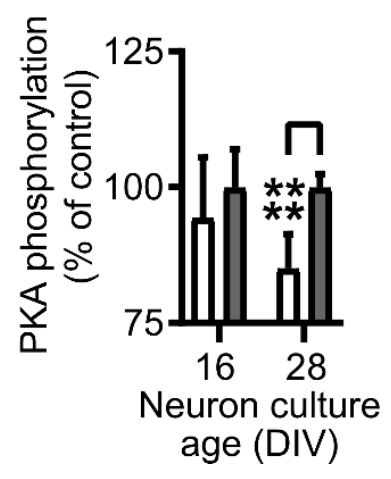

Figure 33. Tau protein overexpression reduces the amount of active pre-synapses, axons and dendrites, as well as the level of cAMP-dependent PKA phosphorylation.

(A-D) Quantification of the amount of pre-synapses (A), axons (B) and dendrites (C) as well as the level of cAMPdependent PKA phosphorylation (D), in the presence of either tau protein overexpression (white bars) or $\gamma$-synuclein overexpression (gray bars). The amount of pre-synapses per $\mathrm{mm}^{2} \pm$ standard deviation is depicted in (A), the percentage of plate surface covered by neurites \pm standard deviation is depicted in (B, C) and the level of PKA phosphorylation as $\%$ of control \pm standard deviation is depicted in $(\mathbf{D})$. $*: \mathrm{p}<0.05 * *: \mathrm{p}<0.01$, ****: $\mathrm{p}<0.0001$. Statistical analysis (used for all subfigures): two-tailed t-test with Welch's correction and a post-hoc power test. Statistical power: $1-\beta>52.6 \%$ for DIV16 and $1-\beta>72.9 \%$ for DIV28 (A), $1-\beta>81.7 \%$ for DIV16 and $1-\beta>100 \%$ for DIV28 (B), $1-\beta>99.9 \%$ (C) $1-\beta>100 \%$ (D). Sample size of independent cultures: 9 (A), 6 for DIV16 and 8 for DIV28 (B), 4 for DIV16 and 6 for DIV28 (C) and 11 for DIV16 and 16 for DIV28 (D).

Indeed, it was observed that tau protein overexpression reduced cell survival by $7-13 \%$ in the presence of Bcl-xL overexpression (Figure 34). Notably however, the loss of axons and pre-synapses was much larger than the loss of cells, with over $50 \%$ of axons and synapses being lost in neurons overexpressing tau protein. These results thus suggested that tau protein overexpression had a cell death independent impact on these structures, although a small portion was also explainable as an outcome of cell death. Notably, the reduction in cytosolic cAMP by tau protein overexpression was also likely cell death independent, as this readout represented the average of all neurons and was thus independent of how many neurons were present in culture. Finally, the loss of dendrites in response to tau protein overexpression was likely induced by cell death in part but not completely, as this loss (19\%) was slightly larger than the observed reduction in cell survival (7-13\%).

Generally, the reduction of the frequency of bursts alongside the loss of synapses and neurites in response to tau protein overexpression permitted two conclusions to be drawn. Firstly, overexpression of tau protein impacted the same mechanisms as the overexpression of $\alpha$-synuclein or the overexpression 
of the $\mathrm{P} 123 \mathrm{H} \beta$-synuclein mutant - it reduced neuronal connectivity and the level of cyclic AMP. As all three also reduced the frequency of network bursting, this reduction was likely caused by an impairment of the same mechanism in the case of all three proteins. Secondly, it was clear that synucleins were not the only proteins that could impact the bursting of neuron networks, as this reduction could also be induced by overexpression of tau protein.

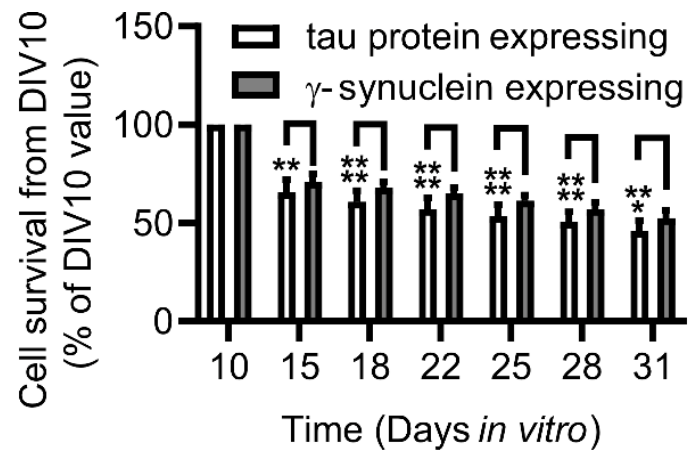

Figure 34. Tau protein overexpression reduces cell survival by $7-13 \%$ in the presence of Bcl-xL overexpression.

Quantification of neuron survival in neurons overexpressing either tau protein (white bars) or $\gamma$-synuclein (gray bars) in the presence of Bcl-xL overexpression. The number of neurons surviving from DIV10 (as \% of the number of neurons at DIV10) \pm standard deviation is depicted. Statistical analysis: two-tailed t-test with Welch's correction and a post-hoc test for statistical power. Statistical power: 85.2\% (DIV16), 99.5\% (DIV19), 99.9\% (DIV22), 99.8\% (DIV25), 99.4\% (DIV28), 97.5\% (DIV31). Sample size: 18 independent cultures. 


\section{Discussion}

In synucleinopathies, a direct connection is thought to exist between the disruptions observed in patients and $\alpha$-synuclein, as evidenced by the existence of synucleinopathy cases that were caused by $\alpha$-synuclein duplication or triplication ${ }^{65,288}$. Further evidence is provided by a variety of experiments in experimental animals that have shown that artificially inducing increased levels of $\alpha$-synuclein expression can lead to disruptions of nervous system functioning, such as neuronal cell death and altered brain rhythmic activity ${ }^{133,156}$.

Of these, the disruption most directly relevant to this project is the reduction of the brain's rhythmic activity frequency in response to increased levels of $\alpha$-synuclein ${ }^{13,133}$. This reduction in rhythmic activity is a relevant property of brain functioning because of two reasons. To begin, in human patients this type of reduction is correlated with the emergence of dementia, such as the dementia associated with late-stage Parkinson's disease or dementia with Lewy bodies ${ }^{3,10,11,127}$. Secondly, rhythmic activity is involved in the regulation of a large variety of brain functions. As an example of this, brain rhythms have been argued to contribute to various cognitive operations ${ }^{1,289}$.

In this project, a study on the impact of increased levels of $\alpha$-synuclein on rhythmic activity was conducted in cultured neurons, which exhibit their own simple type of rhythmic activity, the so-called network bursts. A question could be posed, as to why studying rhythmic activity in this type of model would be relevant, when models for studying this in vivo already exist ${ }^{133}$. The answer to this can be explained in two points.

Firstly, neuronal culture allows for the easy analysis and manipulation of extracellular and intracellular protein species. This is important in the context of $\alpha$-synuclein, as this protein is known to have extracellular forms, which have been suggested in literature to disrupt or otherwise alter neuron functioning ${ }^{135,136,271,273}$.

Secondly, neuronal cultures are easier to probe experimentally than animal models, allowing for easier use of tools such as fluorescent sensors or various types of staining. Notably, several of these methods can also be performed in animal models, but require the use of more complicated methods (e.g. cranial windows for fluorescence imaging), as well as the investment of more time, materials and financial resources for the purchase and maintenance of experimental animals. As such levels of investments are not required in cell culture, it is possible to perform several different analyses as part of the same project. 


\subsection{The overexpression of $\alpha$-synuclein reduces the frequency of neuron network rhythmic activity in vitro.}

Importantly however, for the usability of this model to be established fully, it was necessary for us to determine whether $\alpha$-synuclein changed the properties of rhythmic activity in vitro similarly as it did in vivo. Indeed, it was observed that increased levels of intracellular $\alpha$-synuclein reduced the frequency of neuron network bursting in our neuron culture. This was somewhat similar to the results of an in vivo study by McDowell et al., which observed that lower frequency rhythmic activity became more prevalent in mice expressing increased levels of $\alpha$-synuclein ${ }^{133}$. It was furthermore similar to the study by Caviness et al., which observed that larger levels of phosphorylated $\alpha$-synuclein were correlated with an increased prevalence of lower frequency rhythmic activity in patients ${ }^{13}$.

A basic similarity between the model and the in vivo situation thus existed, as in both cases, an increased level of $\alpha$-synuclein induced a shift of rhythmic activity towards lower frequencies. It is important however to emphasize that the rhythmic activity of the brain is vastly more complicated and diverse than the simple rhythmic activity of neuronal culture. This is because in vivo rhythmic activity is not unitary, but is instead composed of a diverse array of different activities, which summate and are detected as a range of activities with different frequencies by qEEG. This activity furthermore has several different generators, including circuits in both the cortex and the thalamus ${ }^{110}$. In contrast, the bursting inside neuron cultures is a unitary activity, composed of one large repeating depolarization of the neuron culture ${ }^{86}$.

Nevertheless, even with this caveat, it can be argued that enough parallels exist between the in vitro and in vivo activity that it is worthwhile to study rhythmic activity in the context of $\alpha$-synuclein in vitro. Of the different parallels, two in particular are noteworthy. Firstly, both our model and the in vivo model observed a similar type of change in response to increased levels of $\alpha$-synuclein - a shift of the rhythmic activity towards a lower frequency. Secondly, both model systems were composed of the same neuron type - cortical neurons.

Beyond the parallels with in vivo models, studying rhythmic activity using in vitro models also provided unique benefits that were not shared with the in vivo models. Notably, the study of Caviness et al. observed that there was an in vivo correlation between increased levels of phosphorylated $\alpha$-synuclein and a disruption in rhythmic activity. However, this study did not prove that the increased levels of $\alpha$-synuclein caused the disruption - only that these increased levels correlated with the disruption ${ }^{13}$. In contrast, the study by McDowell et al. went further and indicated that $\alpha$-synuclein overexpression in Thy1- $\alpha$-synuclein mice could directly cause the disruption of rhythmic activity ${ }^{133}$. However, this study did not investigate the mechanistic grounds for impacts on rhythmic activity, such as a potential loss in connectivity or a reduction in cytosolic cAMP. The unique advantage of our cell culture model was that these mechanisms would be comparatively easy and cost-efficient to determine in vitro, due to the 
greater invasiveness possible in neuron culture along with the shorter time required to express $\alpha$-synuclein in neurons for observation of rhythmic activity disruptions ${ }^{133}$.

\subsubsection{The observed reduction of rhythmic activity frequency by $\alpha$-synuclein occurs sooner than similar observations in vivo}

Interestingly, the frequency reduction caused by increased levels of $\alpha$-synuclein occurred sooner in our in vitro model than it did in the in vivo studies. Although this could be expected due to the vastly shorter lifespan of neuron cultures compared to living organisms, it is still interesting, as in vivo, increased levels of $\alpha$-synuclein led to rhythmic activity disruptions only after being overexpressed for 9 months or more in the case of experimental mice ${ }^{133}$. In comparison, in vitro increased levels of $\alpha$-synuclein caused a relatively large reduction in as little as 3-5 weeks of overexpression (Figure 18).

This discrepancy can be explained by the fact that $\alpha$-synuclein expression was much higher in this study than in the in vivo studies, being around twenty times higher than levels of normal neuronal expression (Figure 22). In comparison, the method used to overexpress $\alpha$-synuclein in experimental mice induced an expression increase of 1,5-5 fold (depending on brain region) over wild-type expression levels $13,131,133$

\subsection{Extracellular $\alpha$-synuclein was not involved in reducing the frequency of network bursting, contradicting previous results}

Notably, previous studies had observed that application of recombinant $\alpha$-synuclein fibrils could disrupt the functioning of in vitro neuron networks ${ }^{135,136,273}$. Most relevantly to our study, two studies had observed that the application of $0,13-0,50 \mu \mathrm{M}$ recombinant fibrils reduced the frequency of network bursting in neuron culture. Importantly however, these studies used recombinant $\alpha$-synuclein that was aggregated artificially and processed using sonication before use, which cast some doubt on their results 135,136 . This is because the type of $\alpha$-synuclein used in their study does not naturally exist and it is thus questionable whether it is a relevant parallel to $\alpha$-synuclein in patients.

Notably, our study was well-positioned to verify these results using neuronally-produced $\alpha$-synuclein, which arguably provided a closer parallel to patient $\alpha$-synuclein, which is also produced and released by mammalian neurons. Furthermore, our cell culture supernatant contained 0,33-0,36 $\mu \mathrm{M}$ extracellular $\alpha$-synuclein at the later timepoints (Figure 21), which was comparable to the amount used in these studies $(0,13-0,50 \mu \mathrm{M}){ }^{135,136}$. Therefore, our study could be used to investigate whether neuronallyproduced extracellular $\alpha$-synuclein impaired rhythmic activity in the same way as recombinant $\alpha$-synuclein.

In order to answer this question, a medium exchange was carried out where medium containing released synuclein from $\alpha$-synuclein overexpressing neurons was applied to control neurons. Notably however, no reduction in the frequency of rhythmic activity was observed after this medium exchange was 
performed (Figure 23). Other results also showed that extracellular $\alpha$-synuclein did not play a role in regulating the frequency of neuron network bursting. For instance, when an attempt was made to wash off the extracellular species of $\alpha$-synuclein from the neurons using a medium exchange, the neuron network bursting frequency did not change (Figure 24). These observations thus suggested that in our model extracellular $\alpha$-synuclein did not alter the network bursting of in vitro neurons.

As mentioned, this contradiction between our results and previously published results is most likely explained by the different types of samples used in our study compared to those used in previous studies. More specifically, the samples used in our study are of a neuronal origin and undergo folding and production using mammalian cellular machinery. In contrast, the samples used in previous studies were produced recombinantly using E.coli bacteria and aggregated artificially, which could lead to an artificial reduction in burst frequency due to the presence of non-biological types of aggregates or bacterial contaminants ${ }^{135,136}$.

Notably, the presence of bacterial contaminants is a particularly concerning problem, as these have been shown to alter the properties of $\alpha$-synuclein. For instance, LPS species have been shown to alter the aggregation kinetics and the aggregated forms of $\alpha$-synuclein, as well as to change the rate of aggregated $\alpha$-synuclein internalization ${ }^{285,290}$. Beyond this, E.coli bacteria also produce other proteins that have been shown to alter the properties of $\alpha$-synuclein, such as the bacterial amyloid protein curli, which has been shown to enhance $\alpha$-synuclein aggregation ${ }^{291,292}$.

Our results thus suggest that extracellular $\alpha$-synuclein does not disrupt rhythmic activity as has been described in previous studies. Nevertheless, further experiments would need to be performed to confirm this observation. For instance, it would be interesting to determine whether there exist possible differences between neuronally produced and artificially produced extracellular $\alpha$-synuclein, such as on the level of fibril structure or size.

Beyond this, it would also be beneficial to investigate whether neuronally produced synuclein aggregates exhibit other traits that have been shown to exist for recombinant synuclein aggregates. For example, it should be investigated whether neuronally-produced aggregates have the ability to seed intracellular $\alpha$-synuclein aggregation when taken up into neurons using endocytosis ${ }^{273}$. This would be relevant, as this has been observed in the case of recombinant $\alpha$-synuclein. Moreover, this phenomenon has important implications for a variety of larger theories of $\alpha$-synuclein pathology, such as the theory of $\alpha$-synuclein spreading in a prion-like fashion, spreading misfolded synuclein forms as it is spread from neuron to neuron ${ }^{293}$.

Finally, further research on whether recombinantly produced $\alpha$-synuclein contains contaminants would be helpful - although this has been done extensively for lipopolysaccharide endotoxins, it would also need to be done for other potentially relevant contaminants, such as the amyloid fibril forming bacterial protein curli, which - similarly to lipopolysaccharide endotoxins - has been suggested to change the 
aggregation properties of $\alpha$-synuclein ${ }^{286}$. Notably, the studies on curli performed thus far have not investigated its potential role as a contaminant, but have instead analyzed its role as a component of the gut proteome that can alter $\alpha$-synuclein functioning ${ }^{291,292}$.

\subsection{The reduction in burst frequency was correlated with diminishments in structures responsible for neuronal connectivity}

In terms of investigating the reasons why $\alpha$-synuclein reduces the frequency of neuron network bursting, a key hypothesis was that this was mediated by a reduction in connectivity. The basis for this supposition were a variety of studies, including studies using computer modelling, which claimed that connectivity is one of the key parameters for determining the behavior of rhythmic activity in neuronal networks ${ }^{16-20,115}$.

As a readout for determining the level of connectivity, the structural integrity of various structures responsible for neuronal connectivity was investigated - structures such as pre-synapses, axons and dendrites. The level of cAMP was also determined in context of this, as cAMP is a signaling molecule that plays a role in synaptic potentiation signaling pathways and is thus a possible molecular mediator of connectivity ${ }^{4,212}$.

Indeed, when the structural integrity of these structures was evaluated, it was determined that overexpression of either $\alpha$-synuclein, tau protein or the P123H $\beta$-synuclein mutant caused a reduction in at least three of the four analyzed parameters (synapses, axons, dendrites, cAMP). In contrast, overexpression of wild-type $\beta$-synuclein or $\gamma$-synuclein did not change the level of any of the four parameters (Table 5).

The follow-up question to this was whether the reduction in these connecting structures was what caused the reduction of neuron network burst frequency. If this was the case, then a reduction in both connectivity and neuron network burst frequency would have been expected at the same timepoint. Indeed, overexpression of wild-type $\alpha$-synuclein, wild-type tau protein and the $\beta$-synuclein $\mathrm{P} 123 \mathrm{H}$ mutant all caused a substantial reduction of neuron-connecting structures and of neuron network burst frequency at DIV28. In comparison, overexpression of wild-type $\beta$-synuclein induced neither a reduction in neuron network burst frequency nor a reduction in neuron-connecting structures at DIV28 (Table 5).

Thus, a correlation between the reduction of neuron-connecting structures and the reduction of burst frequency was clearly demonstrated. The presence of this correlation was further supported by the fact that the effect size of the connectivity reductions and the effect size of the burst frequency reduction was similar for two of the three proteins. In the case of the tau protein-overexpressing neurons, a $-53 \%$ reduction of axons and a $-56 \%$ reduction of pre-synapses was observed, which was be similar to the $-71 \%$ reduction of burst frequency. In the case of the $\mathrm{P} 123 \mathrm{H} \beta$-synuclein mutant overexpressing neurons, a $-49 \%$ reduction of axons was observed, which was similar to the $-53 \%$ burst frequency reduction. 


\begin{tabular}{|l|c|c|c|c|c|}
\hline Protein tested: & Burst freq. & Synapses & Axons & Dendrites & cAMP/PKA \\
\hline $\begin{array}{l}\alpha \text {-synuclein } \\
\text { (wild-type) }\end{array}$ & $* * * *(-56 \%)$ & $* *(-28 \%)$ & $*(-15 \%)$ & n.s. & $* * * *(-6 \%)$ \\
\hline $\begin{array}{l}\text { tau protein } \\
\text { (wild-type) }\end{array}$ & $* * * *(-71 \%)$ & $*(-53 \%)$ & $*(-56 \%)$ & $* *(-21 \%)$ & $* * *(-15 \%)$ \\
\hline $\begin{array}{l}\beta \text {-synuclein } \\
\text { (P123H mutant) }\end{array}$ & $* * * *(-53 \%)$ & n.s. & $* * * *(-49 \%)$ & $* *(-8 \%)$ & $*(-5 \%)$ \\
\hline $\begin{array}{l}\beta \text {-synuclein } \\
\text { (wild-type) }\end{array}$ & n.s. & n.s. & n.s. & n.s. & n.s. \\
\hline
\end{tabular}

Table 5. Summary of observed connectivity and neuron network bursting differences on DIV28.

Compilation of the various reductions related to either connectivity or neuron network bursting. Statistical significances of the observed differences are indicated. If the result is significant, the percentage size of the reduction compared to $\gamma$-synuclein overexpressing neurons is also indicated. Statistical analysis (used for all subfigures): two-tailed t-test with Welch's correction and a post-hoc power test. *: $\mathrm{p}<0.05, * *$ : $\mathrm{p}<0.01$, $* * *: \mathrm{p}<0.001$, ****: $\mathrm{p}<0.0001$. "n.s." = not significant. "Burst freq." = frequency of network bursting, "Synapses" = amount of functional pre-synapses per $\mathrm{mm}^{2}$, "Axons", "Dendrites" = amount of well surface covered by axons or dendrites. "cAMP/PKA" = level of cAMP-activated PKA phosphorylation. For more details, see Figures 18, 25, 27, 28, 31, 32, 33 .

Nevertheless some discrepancies were also evident. Notably, in the case of the $\alpha$-synuclein overexpressing neurons, no drastic reduction of one single connectivity parameter was observed - burst frequency was reduced by $56 \%$ whereas the highest reduction of a connectivity-related parameter was the reduction in presynapses, which were reduced by $28 \%$. This therefore suggests that although the reduction in burst frequency in neurons overexpressing $\alpha$-synuclein can be explained by a reduction in connecting structures to a significant degree, it is also possible that other disruptions of neuron physiology could be involved, such as alterations in the rate of synaptic or neurotransmitter release.

Furthermore, overexpression of both wild-type $\alpha$-synuclein and mutant P123H $\beta$-synuclein lead to a disruption of three connectivity parameters and not all four. This is the most confusing for the $\mathrm{P} 123 \mathrm{H}$ $\beta$-synuclein mutant, as a reduction was observed in the amount of axons but not in pre-synapses. This is odd, as pre-synapses are structurally connected to axons and thus a reduction of both would be expected. The confusion was strange particularly as overexpression of both wild-type $\alpha$-synuclein and wild-type tau protein led to a reduction of both pre-synapses and axons, whereas the overexpression of wild-type $\beta$-synuclein led to a reduction of neither pre-synapses nor axons (Table 5). Although the cause of this discrepancy is unclear, one possibility is that the neurons compensated for the particular type of axonal lesion induced by the $\mathrm{P} 123 \mathrm{H} \beta$-synuclein mutant, for instance by activating inactive or silent synapses.

Despite the discrepancies however, overall the results suggested that there exists a correlation between connectivity and burst frequency. Nevertheless, future studies should be performed that confirm the presence of this correlation. For instance, connectivity lesions similar to ours could be modelled in in silico models of network bursting in order to determine if this would induce a reduction of network bursting frequency comparable to the one observed in our study. 


\subsubsection{The observed reduction of axons and synapses in response to $\alpha$-synuclein overexpression is coherent with results of previous studies}

Notably, our results on the impact of $\alpha$-synuclein overexpression on neurites and synapses were coherent with previous studies. With regard to axons, numerous studies had observed the presence of axonal pathology and axonal loss in response to increased levels of $\alpha$-synuclein, which was coherent with our observation that $\alpha$-synuclein overexpressing neurons caused a $15 \%$ reduction in the amount of axons (Figure 25) ${ }^{6,28,156,157,294}$. With regard to synapses, a variety of studies had observed disruptions in synaptic functioning and also synaptic loss, which was coherent with our observation that $\alpha$-synuclein caused a -28\% reduction of functional pre-synapses (Figure 25) ${ }^{174-176,179,185-190,295,296}$.

Beyond this, our results were also internally coherent, as a reduction in pre-synapses would be expected to occur alongside a reduction in axons, as the two are structurally linked to one another. Indeed, in our dataset, the overexpression of $\alpha$-synuclein induced both a reduction in axons and in pre-synapses. A slight discrepancy however was that $\alpha$-synuclein overexpression induced an apparent loss of axons $(-15 \%)$ that was lower in magnitude than the apparent loss of synapses (-28\%) (Figure 25). This could be explained by the fact that the number of synapses quantified in this study was not the number of structural synapses but the number of functioning synapses. It is thus possible that an additional mechanism was responsible that involved not a structural destruction of the pre-synapse along with the axon but instead a more subtle functional disruption, such as a reduction of the excitability of the neuron attached to the pre-synapse.

\section{4. $\alpha$-synuclein overexpression reduces the level of cyclic AMP, a signaling molecule which regulates the growth of axons and the formation of synapses}

Notably, our study is the first which has observed that increased levels of $\alpha$-synuclein lead to reduced levels of cAMP in the cytosol. This is relevant as previous studies had only shown that cAMP metabolism was altered in various PD models, but had not tied it specifically to $\alpha$-synuclein ${ }^{34-36,240,241}$. Furthermore, our study also suggested that a reduction in cAMP levels could be one of the explanations behind the reduction of rhythmic activity in response to $\alpha$-synuclein overexpression, suggesting a possible pathway through which the $\alpha$-synuclein induced cAMP reduction could be pathological.

Beyond this however, our study also suggests an interesting hypothesis, namely that $\alpha$-synuclein induces a reduction in pre-synapses and axons by first triggering a reduction in cAMP. This is because a distinguishing feature of cAMP is that cAMP promotes axon growth and regeneration and is involved in synapse formation - it is a regulator of the two of the other connectivity factors observed in our study 297-301. Therefore if $\alpha$-synuclein reduces levels of cAMP, it is plausible that this could trigger a reduction in axons and presynapses (similar in type to the one observed in our study at DIV28).

When compared against our results however, arguments both for and against this hypothesis emerge. To begin, $\alpha$-synuclein does reduce cAMP levels alongside reducing the amount of axons and pre-synapses 
at DIV28 in our cultures (Table 5). However, problematically, no reduction of cAMP levels was observed at DIV16 in our cultures - if a reduction of cAMP were to explain the reduction in axons and pre-synapses, then such a reduction would be expected in earlier culture (Figure 27). By contrast, if cAMP is first reduced in late culture, it is unclear as to how it could reduce the amount of axons and synapses after these structures are already fully formed.

Our results are thus insufficient by themselves to prove that $\alpha$-synuclein overexpression reduces the levels of axons and synapses by first reducing the levels of cAMP. Nevertheless, this remains an attractive possibility that could be researched further in future studies. For instance, it is possible that $\alpha$-synuclein overexpression could reduce cAMP levels or alter cAMP metabolism in cellular subcompartments in early culture.

Two examples of such compartments that could be studied include axonal growth cones and presynapses. Of these, axonal growth cones are important for both axon growth and synapse formation, which are two of the processes that are regulated by cAMP ${ }^{297-301}$. An impairment of cAMP metabolism at this compartment in early culture could thus trigger the reductions of connectivity that were observed in our study. Furthermore, an $\alpha$-synuclein impairment of this compartment is plausible, as growth cones have been shown to natively contain $\alpha$-synuclein ${ }^{302}$. Meanwhile, presynapses would also be an interesting compartment to study as they also contain high levels of $\alpha$-synuclein ${ }^{166,303}$. Furthermore, these compartments are also associated with synaptic potentiation, which is another connectivity-related cellular process regulated by cAMP ${ }^{304}$.

\subsection{Overexpression of the $\mathrm{P} 123 \mathrm{H} \beta$-synuclein mutant reduces the frequency of rhythmic activity by disrupting cellular connectivity, which is similar to the observed impact of $\alpha$-synuclein overexpression}

Notably, $\alpha$-synuclein is not the solitary member of the synuclein family that is associated with neuropathology, as $\beta$-synuclein has been shown to induce cell death when overexpressed in dopaminergic neurons and has also been shown to exhibit altered levels in various cortical areas in DLB patients ${ }^{305-307}$. Beyond this, two $\beta$-synuclein variants (the P123H and V70 $\beta$-synuclein mutants) are associated with DLB and have been shown to be toxic in dopaminergic neurons when overexpressed ${ }^{83,84}$. As these $\beta$-synuclein variants had been observed to have a variety of neurotoxic impacts (e.g. in terms of cell death, mitochondrial disruption), our study decided to investigate whether overexpression of these various $\beta$-synuclein variants impaired neuronal networks in a similar manner as was observed for wild-type $\alpha$-synuclein overexpression ${ }^{84}$. Notably, the study of these $\beta$-synuclein variants was prioritized over the study of $\alpha$-synuclein mutants, as the $\beta$-synuclein variants are much less broadly studied than $\alpha$-synuclein variants, yielding the possibility of discovering novel results.

Indeed, novel results were observed when using one of the $\beta$-synuclein variants, the $\mathrm{P} 123 \mathrm{H} \beta$-synuclein mutant. It was thus observed that the $\mathrm{P} 123 \mathrm{H} \beta$-synuclein mutant was the only synuclein variant aside 
from wild-type $\alpha$-synuclein to substantially reduce the frequency of network bursts when it was overexpressed by cultured neurons. Interestingly, our results suggested that the $\mathrm{P} 123 \mathrm{H} \beta$-synuclein mutant and wild-type $\alpha$-synuclein shared a common pathway for disrupting the functioning of neuron network rhythmic activity, as reductions in connecting structures were observed both in neurons overexpressing the $\mathrm{P} 123 \mathrm{H} \beta$-synuclein mutant as well as wild-type $\alpha$-synuclein. More specifically, a reduction in both axons and levels of cytosolic cyclic AMP were observed in both cases (Table 5).

Our results thus suggest the interesting possibility that a certain degree of commonality exists between the impact of wild-type $\alpha$-synuclein and the impact of the $\mathrm{P} 123 \mathrm{H} \beta$-synuclein mutant. Indeed, commonalities have been suggested before in previous articles, which have shown that the $\mathrm{P} 123 \mathrm{H}$ $\beta$-synuclein mutant's aggregation properties under acidic (but not physiological) conditions as well as the conformation of its $\mathrm{C}$-terminus exhibit similarities to that of $\alpha$-synuclein ${ }^{84,308,309}$. Notably however, the $\mathrm{P} 123 \mathrm{H} \beta$-synuclein mutant also possessed one impact that was unique from the impact of $\alpha$-synuclein. More specifically, overexpression of the $\mathrm{P} 123 \mathrm{H} \beta$-synuclein mutant in neurons reduced the amount of axons by $49 \%$, whereas overexpression of wild-type $\alpha$-synuclein reduced the amount of axons only by $15 \%$ (Table 5 ).

In terms of prospective future studies, it would be interesting to determine if increased levels of the $\mathrm{P} 123 \mathrm{H} \beta$-synuclein mutant induce rhythmic activity disruptions in vivo just as they did in vitro in our study. Indeed, reductions in vivo would be not surprising, as increased levels of $\alpha$-synuclein and tau protein reduced the frequency of rhythmic activity not only in our model but also in various in vivo studies ${ }^{13-15}$. An investigation of the impact of the P123H $\beta$-synuclein mutant could also be relevant to disease, as importantly DLB, the disease associated with the $\mathrm{P} 123 \mathrm{H} \beta$-synuclein mutant, involves rhythmic activity disruptions in patients ${ }^{3}$.

\subsubsection{The non-toxicity of wild-type $\beta$-synuclein and the V70M $\beta$-synuclein mutant is explainable in light of the culture model used in this study}

Notably, in our study increased levels of the P123H $\beta$-synuclein mutant diminished cell survival and also reduced the frequency of neuron network bursting (Figure 28, Figure 29). This was coherent with previous studies, which had similarly shown that increased levels of the $\beta$-synuclein P123H mutant could cause cell death and also induce additional neuronal lesions (such as mitochondrial disruptions) when compared against a non-synuclein control (such as the fluorescent protein EGFP) ${ }^{84}$.

In contrast, our results involving the other two variants are not coherent with previous studies. This is because our results showed that increased levels of the V70M $\beta$-synuclein mutant and wild-type $\beta$-synuclein were not neurotoxic in terms of either cell death or reduction of the frequency of neuron network bursting (Figure 28, Figure 29). Larger-scale studies however contradict these results and indicate that these two variants cause a variety of neurotoxic impacts on neurons, including induction of cell death, disruption of mitochondrial structure and neurite loss ${ }^{78,84}$. Furthermore, these studies 
observed that overexpression of the V70M $\beta$-synuclein mutant was more toxic than overexpression of the $\mathrm{P} 123 \mathrm{H} \beta$-synuclein mutant in certain cases, such as in terms of inducing more neurite loss and a greater disruption of mitochondrial morphology ${ }^{84}$.

This discrepancy between results can be explained by two factors. To begin, dopaminergic neurons were used in several of the experiments that reported increased levels of toxicity in cases of V70M $\beta$-synuclein mutant or wild-type $\beta$-synuclein overexpression ${ }^{78,84}$. This is important, because dopaminergic neurons differ greatly from cortical neurons. For instance, these cells are thought to have a greatly increased energy expenditure due to their sizable axonal arbor, which renders them vulnerable to cellular stressors 310-312. As a result it is plausible they would also be more vulnerable to the impact of neurotoxic proteins, such as the impact of the $\beta$-synuclein variants.

Beyond this, our experiment used a cell culture model, which had been extensively optimized to ensure minimal levels of neurotoxicity in terms of cell death and in terms of the disruption of neuron network bursting. Notably, these optimizations significantly decreased the neurotoxic impact of $\alpha$-synuclein, the most toxic wild-type synuclein - in the presence of the optimizations $\alpha$-synuclein overexpression induced less cell mortality and a smaller reduction on the frequency of network (Figure 15) ${ }^{78}$. It is thus possible that the optimized culture conditions reduced the neurotoxicity of increased levels of wild-type $\beta$-synuclein and the V70 $\beta$-synuclein mutant below the detection threshold.

\subsection{Synucleinopathy-related disruptions of rhythmic activity in vivo could be explained by a reduction of connectivity, as was observed in this study in vitro}

Our study thus suggests that $\alpha$-synuclein overexpression reduces the frequency of rhythmic activity in vitro by reducing connectivity (Table 5). Notably however, the purpose of our study was not to study an in vitro phenomenon but to use the in vitro culture as a model for in vivo rhythmic activity disruptions, such as those observed in synucleinopathies. This therefore invites the question of whether a reduction in connectivity could explain in vivo rhythmic activity disruptions, just as it plausibly explains them in vitro.

On the surface, this is possible, as both disruptions of rhythmic activity and reductions in connecting structures have been reported in synucleinopathy patients. With regard to rhythmic activity, disruptions have been observed using qEEG that take the form of a generalized slowing of cortical rhythmic activity - lower frequency activity becomes more prevalent in relation to higher frequency activity $3,10,313$. Meanwhile, with regard to connecting structures, synucleinopathies are associated with the loss of neurites and synapses in a variety of structures, including the cerebral cortex ${ }_{6,21-23,155,314,315}$.

On a mechanistic level, it is also possible to see how a reduction in connectivity could lead to a disruption in rhythmic activity. This is because rhythmic activity in the brain is dependent on a spread of the rhythm from thalamocortical pacemaking regions to other structures in the cortex, which is mediated by connecting structures - neurites and synapses. Beyond this, generation of the rhythm requires regulation 
and modulation from other brain regions - it is not a single group of neurons which generates a rhythm, but an interconnected network of neurons from different brain regions. This process is also reliant on functioning neuronal connections ${ }^{1,110}$. It is therefore plausible that disruptions of connectivity could lead to disruptions of either rhythmic activity spread or generation.

A relationship between connectivity and rhythmic activity is also suggested by the fact that a worsening of symptoms in late-stage Parkinson's disease leads to both increased disruptions of rhythmic activity and increased loss of connecting structures. More specifically, in patients with Parkinson's disease dementia (PD-D) and Parkinson's disease with mild cognitive impairment (PD-MCI), the generalized slowing of PD-associated qEEG patterns increases in size - lower frequency waves are even more prevalent in PD-D and PD-MCI patients than in PD patients without cognitive symptoms ${ }^{10,127}$. Notably, the transition from PD without cognitive impairment to PD-MCI and PD-D is also associated with a loss of connecting structures, for instance in cortical gray matter, which has been shown to atrophy in increasing amounts in PD-D and PD-MCI patients ${ }^{21-24}$.

Based on these observations, it is thus plausible that a reduction in connectivity could be explained by a reduction in connectivity in vivo. Nevertheless, one flaw of the existing body of data is that it consists of separate studies on either synuclein, connectivity or rhythmic activity, with only a few studies simultaneously studying two topics with the same model (e.g. rhythmic activity in patients and increased levels of $\alpha$-synuclein were studied together by Caviness et al.) ${ }^{13}$.

Crucially, this highlights one of the benefits of our study - due to the simplicity of the in vitro model, it was possible for us to investigate the relationship between $\alpha$-synuclein, connectivity and rhythmic activity in one study. Now that our study has established this relationship in vitro, a logical step in the future would be to establish it in vivo, for instance by simultaneously studying the connection between increased levels of $\alpha$-synuclein, connectivity and rhythmic activity in $\alpha$-synuclein overexpressing animals, such as the Thy $1-\alpha$-synuclein mice used by McDowell et al. ${ }^{133}$.

Beyond this, a more distant goal could be to connect the disruptions in rhythmic activity observed in neuropathological disorders with disruptions of cognition (such as those observed in dementia). Notably, although this would be an extremely complicated task requiring multiple dedicated projects, the basic foundations for such a connection exist, as a variety of studies have claimed that rhythmic activity contributes to several cognitive functions ${ }^{1,289}$. Examples of such cognitive functions include signal filtering, signal coordination and attention ${ }^{289}$.

\subsection{The disruption of rhythmic activity by tau protein overexpression is accompanied by reductions in connectivity}

As mentioned, tau protein was included in our study to test whether a disease-associated protein that was not a member of the synuclein family could disrupt rhythmic activity in vitro in a way similar to $\alpha$-synuclein. Indeed, this was the case, as tau protein overexpression reduced the frequency of rhythmic 
activity (Table 5). Interestingly, the rhythmic activity reduction was also associated with similar reductions in connecting structures, with amounts of axons and synapses being diminished (Table 5). The novel discovery with regard to tau protein is therefore the same as for $\alpha$-synuclein - that tau protein overexpression reduces rhythmic activity in vitro in a process that likely involves connecting structures. Notably, just as in the case of $\alpha$-synuclein, the connection between tau protein overexpression, connectivity reduction and rhythmic activity disruption could also exist in vivo. This is because in the case of $\mathrm{AD}$ patients, there exists a similar kind of basic correlation - both reductions in connecting structures (for instance as part of cortical atrophy) and rhythmic activity are observed in AD patients 14,15,316-318. Furthermore, in AD patients progression of the disease was associated with both an increased level of rhythmic activity disruption as well as increased loss of connecting structures, which was also observed in the case of PD patients ${ }^{10,14,15,127,319,320}$.

Notably, tau protein overexpression reduced the frequency of rhythmic activity by $71 \%$, whereas $\alpha$-synuclein overexpression reduced rhythmic activity only by $56 \%$. On the surface, this suggests that tau protein (and therefore by extension, AD) should be associated with greater disruptions of rhythmic activity than $\alpha$-synuclein (and by extension, PD/DLB). Importantly however, this inference does not match reality. This is because several studies exist which have determined the rhythmic activity of patients suffering from $\mathrm{AD}$ and patients suffering from synucleinopathy associated with cognitive symptoms in parallel - these studies reveal that the disruptions of rhythmic activity in AD patients were not more dramatic than those in synucleinopathy patients with cognitive symptoms ${ }^{3,11,126}$.

Regardless of this however, our study shows that in vitro, tau protein overexpression leads to a reduction in connecting structures, which then likely leads further to a reduction in rhythmic activity frequency. The achievement of our study is therefore determining a relationship between these three parameters in the context of the same study not just for $\alpha$-synuclein but also for tau protein. In the future it would be beneficial, if these connections would now additionally be studied in vivo, for instance in an animal model study that simultaneously images connectivity and rhythmic activity patterns in the presence of increased levels of tau protein.

\subsubsection{The cAMP reduction in response to increased levels of tau overexpression is a novel observation in the context of mammalian neurons}

Notably, our observation that tau overexpression leads to a reduction in cytosolic cyclic AMP is relatively novel. The closest parallel to this is a study conducted in fruit flies, which indicated disruptions of cAMP production in response to dopamine stimulation in Drosophila ventrolateral clock neurons ${ }^{321}$. Nevertheless the large evolutionary distance between Drosophila and humans renders the relevance of this discovery to mammalian biology questionable at face value. It is thus relevant that our study confirmed this phenomenon in specifically mammalian neurons. 
Beyond this, our study also observed a reduction in pre-synapses and neurites in response to tau overexpression. These results however are not novel but instead replicate the results of previous studies, such as the observations of extensive neurite and synapse loss as part of cortical atrophy in Alzheimer's disease, the disorder associated with tau protein ${ }^{316-318}$. Beyond this, parallels also exist with studies conducted in cell culture - a reduction in neurites in response to increased levels of tau was also observed in a study conducted in hippocampal neurons by Verstraelen et al ${ }^{17}$. Notably, Verstraelen et al. also observed a reduction in the frequency of rhythmic activity, although the relationship with connectivity was not as strongly established due to internal inconsistencies in the results - the amount of neurites was reduced in response to increased levels of tau protein, but not the amount of synapses ${ }^{17}$. 


\section{Abstract}

Synucleinopathies are associated with many different types of disruptions, one of which is as a generalized slowing of the brain's rhythmic activity, which co-occurs with dementia in several neurodegenerative disorders. Notably, this slowing has also been connected with increased levels of $\alpha$-synuclein in both patients as well as test animals.

In this study, the impact of increased levels of $\alpha$-synuclein on rhythmic activity was analyzed, with the intention of identifying explanations for the disruptive role of $\alpha$-synuclein. For this, an in vitro model was used to enable the use of a variety of different analytical methods for identifying possible mechanisms.

Indeed, a possible explanation for the $\alpha$-synuclein related rhythmic activity disruption was identified, as $\alpha$-synuclein overexpression not only reduced rhythmic activity in vitro but also simultaneously reduced the amount of structures involved in neuronal connectivity, including axons and synapses. Notably, a novel reduction of the cytosolic levels of cAMP was also observed, with cAMP being a small molecule associated with a variety of signaling pathways, including those related to connectivity.

In addition to this discovery, our study also observed that the rhythmic activity in our in vitro model was not altered when neurons were treated with neuronally-produced $\alpha$-synuclein. This result is relevant as previous studies had claimed that neuronal rhythmic activity could be disrupted when treated with artificially aggregated recombinant $\alpha$-synuclein.

Finally, our study observed that in vitro rhythmic activity was disrupted in cases of tau protein or $\mathrm{P} 123 \mathrm{H}$ $\beta$-synuclein mutant overexpression. Interestingly, the same connectivity disruptions were observed in neurons overexpressing these proteins as in those overexpressing $\alpha$-synuclein, potentially suggesting that the three proteins disrupted rhythmic activity through a common pathway involving a loss of connectivity. 


\section{References}

1 Buzsaki, G. Rhythms of the Brain. (Oxford University Press, 2006).

2 Haider, H. A. et al. Sensitivity of quantitative EEG for seizure identification in the intensive care unit. Neurology 87, 935-944 (2016).

3 Andersson, M., Hansson, O., Minthon, L., Rosén, I. \& Londos, E. Electroencephalogram variability in dementia with Lewy bodies, Alzheimer's disease and controls. Dementia and geriatric cognitive disorders 26, 284-290 (2008).

4 Kandel, E., Schwartz, J., Jessell, T., Siegelbaum, S. \& Hudspeth, A. Principles of Neural Science, Fifth Edition. (McGraw-Hill Professional, 2012).

$5 \quad$ Scheltens, P. et al. Alzheimer's disease. The Lancet 388, 505-517 (2016).

6 Poewe, W. et al. Parkinson disease. Nature reviews Disease primers 3, 17013 (2017).

7 Walker, Z., Possin, K. L., Boeve, B. F. \& Aarsland, D. Lewy body dementias. The Lancet 386, 1683-1697 (2015).

8 Mecarelli, O. Clinical electroencephalography. (Springer, 2019).

9 Kirschstein, T. \& Köhling, R. What is the source of the EEG? Clinical EEG and neuroscience 40, 146-149 (2009).

10 Caviness, J. et al. Differential spectral quantitative electroencephalography patterns between control and Parkinson's disease cohorts. European journal of neurology 23, 387-392 (2016).

11 Bonanni, L. et al. EEG comparisons in early Alzheimer's disease, dementia with Lewy bodies and Parkinson's disease with dementia patients with a 2-year follow-up. Brain 131, 690-705 (2008).

12 Jelic, V. et al. Quantitative electroencephalography in mild cognitive impairment: longitudinal changes and possible prediction of Alzheimer's disease. Neurobiology of aging 21, 533-540 (2000).

13 Caviness, J. N. et al. Cortical phosphorylated $\alpha$-Synuclein levels correlate with brain wave spectra in Parkinson's disease. Movement Disorders 31, 1012-1019 (2016).

14 Jelic, V. et al. EEG slowing and cerebrospinal fluid tau levels in patients with cognitive decline. Neuroreport 9, 157-160 (1998).

15 Smailovic, U. et al. Quantitative EEG power and synchronization correlate with Alzheimer's disease CSF biomarkers. Neurobiology of aging 63, 88-95 (2018).

16 Pasquale, V., Massobrio, P., Bologna, L., Chiappalone, M. \& Martinoia, S. Self-organization and neuronal avalanches in networks of dissociated cortical neurons. Neuroscience 153, 13541369 (2008).

17 Verstraelen, P. et al. Dysregulation of microtubule stability impairs morphofunctional connectivity in primary neuronal networks. Frontiers in cellular neuroscience 11, 173 (2017).

18 Ito, D. et al. Minimum neuron density for synchronized bursts in a rat cortical culture on multielectrode arrays. Neuroscience 171, 50-61 (2010).

19 Hesse, J. \& Gross, T. Self-organized criticality as a fundamental property of neural systems. Frontiers in systems neuroscience 8, 166 (2014).

20 Orlandi, J. G., Soriano, J., Alvarez-Lacalle, E., Teller, S. \& Casademunt, J. Noise focusing and the emergence of coherent activity in neuronal cultures. Nature Physics 9, 582-590 (2013).

21 Song, S. K. et al. The pattern of cortical atrophy in patients with Parkinson's disease according to cognitive status. Movement Disorders 26, 289-296 (2011).

22 Melzer, T. R. et al. Grey matter atrophy in cognitively impaired Parkinson's disease. Journal of Neurology, Neurosurgery \& Psychiatry 83, 188-194 (2012).

23 Chen, F.-X. et al. Gray matter atrophy associated with mild cognitive impairment in Parkinson's disease. Neuroscience Letters 617, 160-165 (2016).

24 Purves, D. Neuroscience, Sixth Edition. (Oxford University Press, 2018).

25 Bozzali, M. et al. White matter damage in Alzheimer's disease assessed in vivo using diffusion tensor magnetic resonance imaging. Journal of Neurology, Neurosurgery \& Psychiatry 72, 742746 (2002). 
Nikolajsen, G. N., Jensen, M. S. \& West, M. J. Cholinergic axon length reduced by 300 meters in the brain of an Alzheimer mouse model. Neurobiology of aging 32, 1927-1931 (2011).

27 Barber, R. et al. White matter lesions on magnetic resonance imaging in dementia with Lewy bodies, Alzheimer's disease, vascular dementia, and normal aging. Journal of Neurology, Neurosurgery \& Psychiatry 67, 66-72 (1999).

28 Koch, J. et al. Alpha-Synuclein affects neurite morphology, autophagy, vesicle transport and axonal degeneration in CNS neurons. Cell death \& disease 6, e1811 (2015).

Terwel, D., Dewachter, I. \& Van Leuven, F. Axonal transport, tau protein, and neurodegeneration in Alzheimer's disease. Neuromolecular medicine 2, 151-165 (2002).

Schulz-Schaeffer, W. J. The synaptic pathology of $\alpha$-synuclein aggregation in dementia with Lewy bodies, Parkinson's disease and Parkinson's disease dementia. Acta neuropathologica 120, 131-143 (2010).

31 Selkoe, D. J. Alzheimer's disease is a synaptic failure. Science 298, 789-791 (2002).

32 Cabin, D. E. et al. Synaptic vesicle depletion correlates with attenuated synaptic responses to prolonged repetitive stimulation in mice lacking $\alpha$-synuclein. Journal of Neuroscience 22, 8797 8807 (2002).

33 Nemani, V. M. et al. Increased expression of $\alpha$-synuclein reduces neurotransmitter release by inhibiting synaptic vesicle reclustering after endocytosis. Neuron 65, 66-79 (2010).

34 Park, H.-Y. et al. Inhibition of adenylyl cyclase type 5 prevents L-DOPA-induced dyskinesia in an animal model of Parkinson's disease. Journal of Neuroscience 34, 11744-11753 (2014). Rangel-Barajas, C. et al. L-DOPA-induced dyskinesia in hemiparkinsonian rats is associated with up-regulation of adenylyl cyclase type V/VI and increased GABA release in the substantia nigra reticulata. Neurobiology of disease 41, 51-61 (2011).

Tong, J. et al. Brain dopamine-stimulated adenylyl cyclase activity in Parkinson's disease, multiple system atrophy, and progressive supranuclear palsy. Annals of Neurology: Official Journal of the American Neurological Association and the Child Neurology Society 55, 125129 (2004).

37 Belmaker, R. et al. The effect of L-dopa and propranolol on human CSF cyclic nucleotides. Psychopharmacology 58, 307-310 (1978).

38 Wahl-Schott, C. \& Biel, M. HCN channels: structure, cellular regulation and physiological function. Cellular and molecular life sciences 66, 470 (2009).

39 Gooch, C. L., Pracht, E. \& Borenstein, A. R. The burden of neurological disease in the United States: a summary report and call to action. Annals of neurology 81, 479-484 (2017).

40 Wimo, A. et al. The worldwide costs of dementia 2015 and comparisons with 2010. Alzheimer's \& Dementia 13, 1-7 (2017).

41 Reitz, C., Brayne, C. \& Mayeux, R. Epidemiology of Alzheimer disease. Nature Reviews Neurology 7, 137 (2011).

42 Sosa-Ortiz, A. L., Acosta-Castillo, I. \& Prince, M. J. Epidemiology of dementias and Alzheimer's disease. Archives of medical research 43, 600-608 (2012).

43 Walker, F. O. Huntington's disease. The Lancet 369, 218-228 (2007).

van Es, M. A. et al. Amyotrophic lateral sclerosis. The Lancet 390, 2084-2098 (2017).

Scarmeas, N. et al. Motor signs during the course of Alzheimer disease. Neurology 63, 975-982 (2004).

46 Kowal, S. L., Dall, T. M., Chakrabarti, R., Storm, M. V. \& Jain, A. The current and projected economic burden of Parkinson's disease in the United States. Movement Disorders 28, 311-318 (2013).

47 Kalia, L. V. \& Lang, A. E. Parkinson's disease. The Lancet 386, 896-912 (2015).

48 Schapira, A. H., Chaudhuri, K. R. \& Jenner, P. Non-motor features of Parkinson disease. Nature Reviews Neuroscience 18, 435 (2017).

49 Gomperts, S. N. Lewy body dementias: dementia with Lewy bodies and Parkinson disease dementia. Continuum: Lifelong Learning in Neurology 22, 435 (2016).

50 McKeith, I. G. et al. Diagnosis and management of dementia with Lewy bodies: Fourth consensus report of the DLB Consortium. Neurology 89, 88-100 (2017).

51 Burns, A. \& Iliffe, S. Alzheimer's disease. BMJ 338, b158, doi:10.1136/bmj.b158 (2009).

52 Sevigny, J. et al. The antibody aducanumab reduces A $\beta$ plaques in Alzheimer's disease. Nature 537, 50 (2016). 
Ngandu, T. et al. A 2 year multidomain intervention of diet, exercise, cognitive training, and vascular risk monitoring versus control to prevent cognitive decline in at-risk elderly people (FINGER): a randomised controlled trial. The Lancet 385, 2255-2263 (2015).

54 Gauthier, S. et al. Mild cognitive impairment. Lancet 367, 1262-1270, doi:10.1016/s01406736(06)68542-5 (2006).

55 Ritchie, K. Mild cognitive impairment: an epidemiological perspective. Dialogues in clinical neuroscience 6, 401 (2004).

56 Kurosinski, P., Guggisberg, M. \& Götz, J. Alzheimer's and Parkinson's disease-overlapping or synergistic pathologies? Trends in molecular medicine 8, 3-5 (2002).

57 Kotzbauer, P. T., Trojanowski, J. Q. \& Lee, V. M.-Y. Lewy body pathology in Alzheimer's disease. Journal of Molecular Neuroscience 17, 225-232 (2001).

58 Zhang, X. et al. Tau pathology in Parkinson's Disease. Frontiers in neurology 9, 809 (2018).

59 George, J. M. The synucleins. Genome Biology 3, reviews3002.3001, doi:10.1186/gb-2001-31-reviews3002 (2001).

60 Iwai, A. et al. The precursor protein of non-A $\beta$ component of Alzheimer's disease amyloid is a presynaptic protein of the central nervous system. Neuron 14, 467-475 (1995).

61 Kokhan, V., Afanasyeva, M. \& Van'kin, G. $\alpha$-Synuclein knockout mice have cognitive impairments. Behavioural brain research 231, 226-230 (2012).

62 Vilar, M. et al. The fold of $\alpha$-synuclein fibrils. Proceedings of the National Academy of Sciences 105, 8637-8642 (2008).

63 Uversky, V. N. Neuropathology, biochemistry, and biophysics of $\alpha$-synuclein aggregation. Journal of neurochemistry 103, 17-37 (2007).

64 Emamzadeh, F. N. Alpha-synuclein structure, functions, and interactions. J Res Med Sci 21, 2929, doi:10.4103/1735-1995.181989 (2016).

65 Singleton, A. et al. $\alpha$-Synuclein locus triplication causes Parkinson's disease. science 302, 841841 (2003).

66 Conway, K. A., Harper, J. D. \& Lansbury, P. T. Accelerated in vitro fibril formation by a mutant $\alpha$-synuclein linked to early-onset Parkinson disease. Nature medicine 4, 1318-1320 (1998).

67 Spillantini, M. G. et al. $\alpha$-Synuclein in Lewy bodies. Nature 388, 839 (1997).

68 Lowe, J. et al. Ubiquitin is a common factor in intermediate filament inclusion bodies of diverse type in man, including those of Parkinson's disease, Pick's disease, and Alzheimer's disease, as well as Rosenthal fibres in cerebellar astrocytomas, cytoplasmic bodies in muscle, and mallory bodies in alcoholic liver disease. The Journal of pathology 155, 9-15 (1988).

69 Wakabayashi, K. et al. The Lewy body in Parkinson's disease and related neurodegenerative disorders. Molecular neurobiology 47, 495-508 (2013).

70 Braak, H. et al. Staging of the intracerebral inclusion body pathology associated with idiopathic Parkinson's disease (preclinical and clinical stages). Journal of neurology 249, iii1-iii5 (2002).

71 Parkkinen, L., Pirttilä, T. \& Alafuzoff, I. Applicability of current staging/categorization of $\alpha$ synuclein pathology and their clinical relevance. Acta neuropathologica 115, 399-407 (2008).

72 Duffy, P. E. \& Tennyson, V. M. Phase and electron microscopic observations of Lewy bodies and melanin granules in the substantia nigra and locus caeruleus in Parkinson's disease. Journal of Neuropathology \& Experimental Neurology 24, 398-414 (1965).

73 Kanazawa, T. et al. Three-Layered Structure Shared Between Lewy Bodies and Lewy Neurites-Three-Dimensional Reconstruction of Triple-Labeled Sections. Brain pathology 18, 415-422 (2008).

74 Chandra, S. et al. Double-knockout mice for $\alpha$-and $\beta$-synucleins: effect on synaptic functions. Proceedings of the National Academy of Sciences 101, 14966-14971 (2004).

75 Jenco, J. M., Rawlingson, A., Daniels, B. \& Morris, A. J. Regulation of phospholipase D2: selective inhibition of mammalian phospholipase $\mathrm{D}$ isoenzymes by $\alpha$-and $\beta$-synucleins. Biochemistry 37, 4901-4909 (1998).

76 Lavedan, C. The synuclein family. Genome research 8, 871-880 (1998).

77 Biere, A. L. et al. Parkinson's disease-associated $\alpha$-synuclein is more fibrillogenic than $\beta$-and $\gamma$ synuclein and cannot cross-seed its homologs. Journal of Biological Chemistry 275, 3457434579 (2000).

78 Taschenberger, G. et al. $\beta$-synuclein aggregates and induces neurodegeneration in dopaminergic neurons. Annals of neurology 74, 109-118 (2013). 
Xia, Q. et al. Proteomic identification of novel proteins associated with Lewy bodies. Frontiers in bioscience: a journal and virtual library 13, 3850 (2008).

80 Shaltiel-Karyo, R. et al. Inhibiting $\alpha$-synuclein oligomerization by stable cell-penetrating $\beta$ synuclein fragments recovers phenotype of Parkinson's disease model flies. PLoS One 5 (2010).

81 Brown, J. W. et al. $\beta$-Synuclein suppresses both the initiation and amplification steps of $\alpha$ synuclein aggregation via competitive binding to surfaces. Scientific reports 6, 36010 (2016).

82 Windisch, M., Hutter-Paier, B., Schreiner, E. \& Wronski, R. $\beta$-synuclein-derived peptides with neuroprotective activity. Journal of Molecular Neuroscience 24, 155-165 (2004).

83 Ohtake, H. et al. Beta-synuclein gene alterations in dementia with Lewy bodies. Neurology 63, 805-811, doi:10.1212/01.wnl.0000139870.14385.3c (2004).

84 Psol, M. Neurotoxicity and aggregation of $\beta$-synuclein and its P123H and V70M mutants associated with dementia with Lewy bodies, Georg-August-University, (2018).

85 Surgucheva, I., McMahon, B. \& Surguchov, A. $\gamma$-synuclein has a dynamic intracellular localization. Cell motility and the cytoskeleton 63, 447-458 (2006).

86 Robinson, H. et al. Periodic synchronized bursting and intracellular calcium transients elicited by low magnesium in cultured cortical neurons. Journal of neurophysiology 70, 1606-1616 (1993).

87 Millership, S. et al. Increased lipolysis and altered lipid homeostasis protect $\gamma$-synuclein-null mutant mice from diet-induced obesity. Proceedings of the National Academy of Sciences 109, 20943-20948 (2012).

88 Ahmad, M., Attoub, S., Singh, M. N., Martin, F. L. \& El-Agnaf, O. M. $\gamma$-Synuclein and the progression of cancer. The FASEB Journal 21, 3419-3430 (2007).

89 Clayton, D. F. \& George, J. M. The synucleins: a family of proteins involved in synaptic function, plasticity, neurodegeneration and disease. Trends in neurosciences 21, 249-254 (1998).

90 Nishioka, K. et al. Association of $\alpha$-, $\beta$-, and $\gamma$-synuclein with diffuse Lewy body disease. Archives of neurology 67, 970-975 (2010).

91 Drubin, D. G. \& Kirschner, M. W. Tau protein function in living cells. The Journal of cell biology 103, 2739-2746 (1986).

92 Morris, M. et al. Age-appropriate cognition and subtle dopamine-independent motor deficits in aged tau knockout mice. Neurobiology of aging 34, 1523-1529 (2013).

93 Dawson, H. N. et al. Inhibition of neuronal maturation in primary hippocampal neurons from $\tau$ deficient mice. Journal of cell science 114, 1179-1187 (2001).

94 Spillantini, M. G. \& Goedert, M. Tau protein pathology in neurodegenerative diseases. Trends in neurosciences 21, 428-433 (1998).

95 Alonso, A. d. C., Grundke-Iqbal, I. \& Iqbal, K. Alzheimer's disease hyperphosphorylated tau sequesters normal tau into tangles of filaments and disassembles microtubules. Nature medicine 2, 783 (1996).

96 Liu, F. et al. Role of glycosylation in hyperphosphorylation of tau in Alzheimer's disease. FEBS letters 512, 101-106 (2002).

97 Armstrong, R. A. Plaques and tangles and the pathogenesis of Alzheimer's disease. Folia Neuropathologica 44 (2006).

98 Goedert, M., Sisodia, S. S. \& Price, D. L. Neurofibrillary tangles and $\beta$-amyloid deposits in Alzheimer's disease. Current opinion in neurobiology 1, 441-447 (1991).

99 Wiśniewski, H. M., Narang, H. K. \& Terry, R. D. Neurofibrillary tangles of paired helical filaments. Journal of the neurological sciences 27, 173-181 (1976).

100 Priller, C. et al. Synapse formation and function is modulated by the amyloid precursor protein. Journal of Neuroscience 26, 7212-7221 (2006).

101 Busciglio, J., Lorenzo, A., Yeh, J. \& Yankner, B. A. $\beta$-Amyloid fibrils induce tau phosphorylation and loss of microtubule binding. Neuron 14, 879-888 (1995).

102 Balleza-Tapia, H. \& Pena, F. Pharmacology of the intracellular pathways activated by amyloid beta protein. Mini reviews in medicinal chemistry 9, 724-740 (2009).

103 Dawson, G. et al. Age-related cognitive deficits, impaired long-term potentiation and reduction in synaptic marker density in mice lacking the $\beta$-amyloid precursor protein. Neuroscience $\mathbf{9 0}$, 1-13 (1999). 
104 Atwood, C. S., Martins, R. N., Smith, M. A. \& Perry, G. Senile plaque composition and posttranslational modification of amyloid- $\beta$ peptide and associated proteins. Peptides 23, 1343 1350 (2002).

105 Chiti, F. \& Dobson, C. M. Protein misfolding, functional amyloid, and human disease. Annu. Rev. Biochem. 75, 333-366 (2006).

106 Dickson, D. W. The pathogenesis of senile plaques. Journal of Neuropathology \& Experimental Neurology 56, 321-339 (1997).

107 Ben-Ari, Y. Developing networks play a similar melody. Trends in neurosciences 24, 353-360 (2001).

108 Garaschuk, O., Linn, J., Eilers, J. \& Konnerth, A. Large-scale oscillatory calcium waves in the immature cortex. Nature neuroscience 3, 452-459 (2000).

109 Sipilä, S. T., Huttu, K., Soltesz, I., Voipio, J. \& Kaila, K. Depolarizing GABA Acts on Intrinsically Bursting Pyramidal Neurons to Drive Giant Depolarizing Potentials in the Immature Hippocampus. The Journal of Neuroscience 25, 5280 (2005).

110 Crunelli, V. et al. Dual function of thalamic low-vigilance state oscillations: rhythm-regulation and plasticity. Nature Reviews Neuroscience 19, 107 (2018).

111 Lörincz, M. L. et al. A distinct class of slow $(\sim 0.2-2 \mathrm{~Hz})$ intrinsically bursting layer 5 pyramidal neurons determines UP/DOWN state dynamics in the neocortex. $J$ Neurosci 35, 5442-5458 (2015).

112 Sanchez-Vives, M. V. \& McCormick, D. A. Cellular and network mechanisms of rhythmic recurrent activity in neocortex. Nature Neuroscience 3, 1027-1034, doi:10.1038/79848 (2000).

113 Tolö, J. et al. Pathophysiological Consequences of Neuronal $\alpha$-Synuclein Overexpression: Impacts on Ion Homeostasis, Stress Signaling, Mitochondrial Integrity, and Electrical Activity. Frontiers in molecular neuroscience 11, 49 (2018).

114 De Lima, A. D., Merten, M. D. \& Voigt, T. Neuritic differentiation and synaptogenesis in serum-free neuronal cultures of the rat cerebral cortex. Journal of Comparative Neurology 382, 230-246 (1997).

115 Gritsun, T. A., Le Feber, J., Stegenga, J. \& Rutten, W. L. Network bursts in cortical cultures are best simulated using pacemaker neurons and adaptive synapses. Biological cybernetics 102, 293-310 (2010).

116 Voigt, T., Baier, H. \& de Lima, A. D. Synchronization of neuronal activity promotes survival of individual rat neocortical neurons in early development. European Journal of Neuroscience 9, 990-999 (1997).

117 Beggs, J. M. \& Plenz, D. Neuronal avalanches in neocortical circuits. Journal of neuroscience 23, 11167-11177 (2003).

118 Opitz, T., De Lima, A. D. \& Voigt, T. Spontaneous development of synchronous oscillatory activity during maturation of cortical networks in vitro. Journal of neurophysiology 88, 21962206 (2002).

119 Voigt, T., Opitz, T. \& de Lima, A. D. Synchronous oscillatory activity in immature cortical network is driven by GABAergic preplate neurons. Journal of Neuroscience 21, 8895-8905 (2001).

120 McCormick, D. A. \& Huguenard, J. R. A model of the electrophysiological properties of thalamocortical relay neurons. Journal of neurophysiology 68, 1384-1400 (1992).

121 Andreasen, M., Skov, J. \& Nedergaard, S. Inwardly rectifying K+ (Kir) channels antagonize ictal-like epileptiform activity in area CA1 of the rat hippocampus. Hippocampus 17, 10371048 (2007).

122 Klueva, J., de Lima, A. D., Meis, S., Voigt, T. \& Munsch, T. Hyperpolarization-activated cation current contributes to spontaneous network activity in developing neocortical cultures. Neurosignals 20, 35-47 (2012).

123 Maeda, E., Robinson, H. \& Kawana, A. The mechanisms of generation and propagation of synchronized bursting in developing networks of cortical neurons. Journal of Neuroscience 15, 6834-6845 (1995).

124 Buccino, A. P. et al. Combining biophysical modeling and deep learning for multielectrode array neuron localization and classification. Journal of neurophysiology 120, 1212-1232 (2018).

125 Akerboom, J. et al. Genetically encoded calcium indicators for multi-color neural activity imaging and combination with optogenetics. Frontiers in molecular neuroscience 6, 2 (2013). 
126 Fonseca, L. C., Tedrus, G. M., Carvas, P. N. \& Machado, E. C. Comparison of quantitative EEG between patients with Alzheimer's disease and those with Parkinson's disease dementia. Clinical Neurophysiology 124, 1970-1974 (2013).

127 Caviness, J. N. et al. Longitudinal EEG changes correlate with cognitive measure deterioration in Parkinson's disease. Journal of Parkinson's disease 5, 117-124 (2015).

128 Klassen, B. et al. Quantitative EEG as a predictive biomarker for Parkinson disease dementia. Neurology 77, 118-124 (2011).

129 Bonanni, L. et al. Quantitative electroencephalogram utility in predicting conversion of mild cognitive impairment to dementia with Lewy bodies. Neurobiology of aging 36, 434-445 (2015).

130 Lopez, O. et al. EEG spectral abnormalities and psychosis as predictors of cognitive and functional decline in probable Alzheimer's disease. Neurology 48, 1521-1525 (1997).

131 Chesselet, M. F. et al. A progressive mouse model of Parkinson's disease: the Thy1-aSyn ("Line 61") mice. Neurotherapeutics : the journal of the American Society for Experimental NeuroTherapeutics 9, 297-314, doi:10.1007/s13311-012-0104-2 (2012).

132 Rockenstein, E. et al. Differential neuropathological alterations in transgenic mice expressing $\alpha$-synuclein from the platelet-derived growth factor and Thy-1 promoters. Journal of neuroscience research $\mathbf{6 8}, 568-578$ (2002).

133 McDowell, K. A., Shin, D., Roos, K. P. \& Chesselet, M.-F. Sleep dysfunction and EEG alterations in mice overexpressing alpha-synuclein. Journal of Parkinson's disease 4, 531-539 (2014).

134 Morris, M. et al. Network dysfunction in $\alpha$-synuclein transgenic mice and human Lewy body dementia. Annals of clinical and translational neurology 2, 1012-1028 (2015).

135 Froula, J. M. et al. $\alpha$-Synuclein fibril-induced paradoxical structural and functional defects in hippocampal neurons. Acta Neuropathologica Communications 6, 35, doi:10.1186/s40478-0180537-x (2018).

136 Gribaudo, S. et al. Propagation of $\alpha$-Synuclein Strains within Human Reconstructed Neuronal Network. Stem Cell Reports 12, 230-244 (2019).

137 Raichle, M. E. The restless brain: how intrinsic activity organizes brain function. Philosophical Transactions of the Royal Society B: Biological Sciences 370, 20140172 (2015).

138 Van Den Heuvel, M. P. \& Pol, H. E. H. Exploring the brain network: a review on resting-state fMRI functional connectivity. European neuropsychopharmacology 20, 519-534 (2010).

139 Huettel, S., Song, A. \& McCarthy, G. Functional Magnetic Resonance Imaging, Second Edition. (2008).

140 Stebbins, G. et al. Altered cortical visual processing in PD with hallucinations: an fMRI study. Neurology 63, 1409-1416 (2004).

141 Dubbelink, K. T. O. et al. Functional connectivity and cognitive decline over 3 years in Parkinson disease. Neurology 83, 2046-2053 (2014).

142 Tessitore, A. et al. Default-mode network connectivity in cognitively unimpaired patients with Parkinson disease. Neurology 79, 2226-2232 (2012).

143 Peraza, L. R. et al. fMRI resting state networks and their association with cognitive fluctuations in dementia with Lewy bodies. NeuroImage: clinical 4, 558-565 (2014).

144 Franciotti, R. et al. Default network is not hypoactive in dementia with fluctuating cognition: an Alzheimer disease/dementia with Lewy bodies comparison. Neurobiology of aging $\mathbf{3 4}, 1148$ 1158 (2013).

145 Taylor, J.-P. et al. Visual cortex in dementia with Lewy bodies: magnetic resonance imaging study. The British Journal of Psychiatry 200, 491-498 (2012).

146 Agosta, F. et al. Resting state fMRI in Alzheimer's disease: beyond the default mode network. Neurobiology of Aging 33, 1564-1578 (2012).

147 Greicius, M. D., Srivastava, G., Reiss, A. L. \& Menon, V. Default-mode network activity distinguishes Alzheimer's disease from healthy aging: evidence from functional MRI. Proceedings of the National Academy of Sciences 101, 4637-4642 (2004).

148 Zhang, H.-Y. et al. Resting brain connectivity: changes during the progress of Alzheimer disease. Radiology 256, 598-606 (2010).

149 Wang, L. et al. Changes in hippocampal connectivity in the early stages of Alzheimer's disease: evidence from resting state fMRI. Neuroimage 31, 496-504 (2006). 
150 Golby, A. et al. Memory encoding in Alzheimer's disease: an fMRI study of explicit and implicit memory. Brain 128, 773-787 (2005).

151 Bagetta, V., Ghiglieri, V., Sgobio, C., Calabresi, P. \& Picconi, B. (Portland Press Ltd., 2010).

152 Burke, R. E. \& O'Malley, K. Axon degeneration in Parkinson's disease. Experimental neurology 246, $72-83$ (2013).

153 Schulz-Schaeffer, W. J. Is cell death primary or secondary in the pathophysiology of idiopathic Parkinson's disease? Biomolecules 5, 1467-1479 (2015).

154 Tagliaferro, P. \& Burke, R. E. Retrograde axonal degeneration in Parkinson disease. Journal of Parkinson's disease 6, 1-15 (2016).

155 Overk, C. R. \& Masliah, E. Pathogenesis of synaptic degeneration in Alzheimer's disease and Lewy body disease. Biochemical pharmacology 88, 508-516 (2014).

156 Taschenberger, G. et al. Aggregation of $\alpha$ Synuclein promotes progressive in vivo neurotoxicity in adult rat dopaminergic neurons. Acta Neuropathologica 123, 671-683, doi:10.1007/s00401011-0926-8 (2012).

157 Games, D. et al. Axonopathy in an $\alpha$-Synuclein Transgenic Model of Lewy Body Disease Is Associated with Extensive Accumulation of C-Terminal-Truncated $\alpha$-Synuclein. The American journal of pathology 182, 940-953 (2013).

158 Volpicelli-Daley, L. A. Effects of $\alpha$-synuclein on axonal transport. Neurobiology of disease 105, 321-327 (2017).

159 Carnwath, T., Mohammed, R. \& Tsiang, D. The direct and indirect effects of $\alpha$-synuclein on microtubule stability in the pathogenesis of Parkinson's disease. Neuropsychiatric disease and treatment 14, 1685 (2018).

160 Prots, I. et al. $\alpha$-Synuclein oligomers induce early axonal dysfunction in human iPSC-based models of synucleinopathies. Proceedings of the National Academy of Sciences 115, 7813-7818 (2018).

161 Ettle, B. et al. $\alpha$-Synuclein-induced myelination deficit defines a novel interventional target for multiple system atrophy. Acta neuropathologica 132, 59-75 (2016).

162 Grigoletto, J. et al. Higher levels of myelin phospholipids in brains of neuronal $\alpha$-Synuclein transgenic mice precede myelin loss. Acta neuropathologica communications 5, 1-16 (2017).

163 Dryanovski, D. I. et al. Calcium entry and $\alpha$-synuclein inclusions elevate dendritic mitochondrial oxidant stress in dopaminergic neurons. Journal of Neuroscience 33, 1015410164 (2013).

164 Blumenstock, S. et al. Seeding and transgenic overexpression of alpha-synuclein triggers dendritic spine pathology in the neocortex. EMBO molecular medicine 9, 716-731 (2017).

165 Wagner, L., Nathwani, S., Ten Eyck, T. \& Aldridge, G. Local cortical overexpression of human wild-type alpha-synuclein leads to increased dendritic spine density in mouse. Neuroscience Letters, 135051 (2020).

166 Withers, G. S., George, J. M., Banker, G. A. \& Clayton, D. F. Delayed localization of synelfin (synuclein, NACP) to presynaptic terminals in cultured rat hippocampal neurons. Developmental brain research 99, 87-94 (1997).

167 Calo, L., Wegrzynowicz, M., Santivañez-Perez, J. \& Grazia Spillantini, M. Synaptic failure and $\alpha$-synuclein. Movement Disorders 31, 169-177 (2016).

168 Kubo, S.-i. et al. A combinatorial code for the interaction of $\alpha$-synuclein with membranes. Journal of Biological Chemistry 280, 31664-31672 (2005).

169 Fortin, D. L. et al. Lipid rafts mediate the synaptic localization of $\alpha$-synuclein. Journal of Neuroscience 24, 6715-6723 (2004).

170 Burré, J., Sharma, M. \& Südhof, T. C. $\alpha$-Synuclein assembles into higher-order multimers upon membrane binding to promote SNARE complex formation. Proceedings of the National Academy of Sciences 111, E4274-E4283 (2014).

171 Burré, J. et al. Properties of native brain $\alpha$-synuclein. Nature 498, E4-E6 (2013).

172 Perrin, R. J., Woods, W. S., Clayton, D. F. \& George, J. M. Exposure to long chain polyunsaturated fatty acids triggers rapid multimerization of synucleins. Journal of Biological Chemistry 276, 41958-41962 (2001).

173 Iljina, M. et al. Arachidonic acid mediates the formation of abundant alpha-helical multimers of alpha-synuclein. Scientific reports 6, 33928 (2016). 
174 Lou, X., Kim, J., Hawk, B. J. \& Shin, Y.-K. $\alpha$-Synuclein may cross-bridge v-SNARE and acidic phospholipids to facilitate SNARE-dependent vesicle docking. Biochemical Journal 474, 2039 2049 (2017).

175 Burré, J. et al. $\alpha$-Synuclein promotes SNARE-complex assembly in vivo and in vitro. Science 329, 1663-1667 (2010).

176 Chandra, S., Gallardo, G., Fernández-Chacón, R., Schlüter, O. M. \& Südhof, T. C. $\alpha$-Synuclein cooperates with CSP $\alpha$ in preventing neurodegeneration. Cell 123, 383-396 (2005).

177 Bellani, S. et al. The regulation of synaptic function by $\alpha$-synuclein. Communicative \& integrative biology 3, 106-109 (2010).

178 Denker, A. \& Rizzoli, S. O. Synaptic vesicle pools: an update. Front Synaptic Neurosci 2, 135135, doi:10.3389/fnsyn.2010.00135 (2010).

179 Murphy, D. D., Rueter, S. M., Trojanowski, J. Q. \& Lee, V. M.-Y. Synucleins are developmentally expressed, and $\alpha$-synuclein regulates the size of the presynaptic vesicular pool in primary hippocampal neurons. Journal of Neuroscience 20, 3214-3220 (2000).

180 Sankaranarayanan, S., Atluri, P. P. \& Ryan, T. A. Actin has a molecular scaffolding, not propulsive, role in presynaptic function. Nature neuroscience 6, 127-135 (2003).

181 Sousa, V. L. et al. $\alpha$-Synuclein and its A30P mutant affect actin cytoskeletal structure and dynamics. Molecular biology of the cell 20, 3725-3739 (2009).

182 Levitan, E. S. Signaling for vesicle mobilization and synaptic plasticity. Molecular neurobiology 37, 39 (2008).

183 Scott, D. \& Roy, S. $\alpha$-Synuclein inhibits intersynaptic vesicle mobility and maintains recyclingpool homeostasis. Journal of Neuroscience 32, 10129-10135 (2012).

184 Sun, J. et al. Functional cooperation of $\alpha$-synuclein and VAMP2 in synaptic vesicle recycling. Proceedings of the National Academy of Sciences 116, 11113-11115 (2019).

185 Abeliovich, A. et al. Mice lacking $\alpha$-synuclein display functional deficits in the nigrostriatal dopamine system. Neuron 25, 239-252 (2000).

186 Al-Wandi, A. et al. Absence of $\alpha$-synuclein affects dopamine metabolism and synaptic markers in the striatum of aging mice. Neurobiology of aging 31, 796-804 (2010).

187 Anwar, S. et al. Functional alterations to the nigrostriatal system in mice lacking all three members of the synuclein family. Journal of Neuroscience 31, 7264-7274 (2011).

188 Senior, S. L. et al. Increased striatal dopamine release and hyperdopaminergic-like behaviour in mice lacking both alpha-synuclein and gamma-synuclein. European Journal of Neuroscience 27, 947-957 (2008).

189 Lundblad, M., Decressac, M., Mattsson, B. \& Björklund, A. Impaired neurotransmission caused by overexpression of $\alpha$-synuclein in nigral dopamine neurons. Proceedings of the National Academy of Sciences 109, 3213-3219 (2012).

190 Daher, J. P. L. et al. Conditional transgenic mice expressing C-terminally truncated human $\alpha$ synuclein ( $\alpha$ Syn119) exhibit reduced striatal dopamine without loss of nigrostriatal pathway dopaminergic neurons. Molecular neurodegeneration 4, 34 (2009).

191 Guatteo, E. et al. Functional alterations of the dopaminergic and glutamatergic systems in spontaneous $\alpha$-synuclein overexpressing rats. Experimental neurology 287, 21-33 (2017).

192 Kramer, M. L. \& Schulz-Schaeffer, W. J. Presynaptic alpha-synuclein aggregates, not Lewy bodies, cause neurodegeneration in dementia with Lewy bodies. J Neurosci 27, 1405-1410, doi:10.1523/JNEUROSCI.4564-06.2007 (2007).

193 Tanji, K. et al. Proteinase K-resistant $\alpha$-synuclein is deposited in presynapses in human Lewy body disease and A53T $\alpha$-synuclein transgenic mice. Acta neuropathologica 120, 145-154 (2010).

194 Orimo, S. et al. Axonal $\alpha$-synuclein aggregates herald centripetal degeneration of cardiac sympathetic nerve in Parkinson's disease. Brain 131, 642-650 (2008).

195 Kanaan, N. M. et al. Axonal degeneration in Alzheimer's disease: when signaling abnormalities meet the axonal transport system. Experimental neurology 246, 44-53 (2013).

196 Hardiman, O., Doherty, C. P., Elamin, M. \& Bede, P. Neurodegenerative disorders. (Springer, 2011).

197 Tönnies, E. \& Trushina, E. Oxidative stress, synaptic dysfunction, and Alzheimer's disease. Journal of Alzheimer's Disease 57, 1105-1121 (2017). 
198 Ávila, J., Lim, F., Moreno, F., Belmonte, C. \& Cuello, A. C. Tau function and dysfunction in neurons. Molecular neurobiology 25, 213-231 (2002).

199 Spittaels, K. et al. Prominent axonopathy in the brain and spinal cord of transgenic mice overexpressing four-repeat human tau protein. The American journal of pathology 155, 21532165 (1999).

200 Adams, S. J. et al. Overexpression of wild-type murine tau results in progressive tauopathy and neurodegeneration. The American journal of pathology 175, 1598-1609 (2009).

201 Yuan, A., Kumar, A., Peterhoff, C., Duff, K. \& Nixon, R. A. Axonal transport rates in vivo are unaffected by tau deletion or overexpression in mice. Journal of Neuroscience 28, 1682-1687 (2008).

202 Pooler, A. M., Noble, W. \& Hanger, D. P. A role for tau at the synapse in Alzheimer's disease pathogenesis. Neuropharmacology 76, 1-8 (2014).

203 Arendt, T., Stieler, J. T. \& Holzer, M. Tau and tauopathies. Brain research bulletin 126, 238292 (2016).

204 Takashima, A. Tauopathies and tau oligomers. Journal of Alzheimer's Disease 37, 565-568 (2013).

205 Zhou, L. et al. Tau association with synaptic vesicles causes presynaptic dysfunction. Nature communications 8, 1-13 (2017).

206 Nelson, D. L. \& Cox, M. M. Lehninger Principles of Biochemistry, Sixth Edition. (MacMillan, 2013).

207 Fimia, G. M. \& Sassone-Corsi, P. Cyclic AMP signalling. Journal of cell science 114, 1971$1972(2001)$.

208 Vizi, E. S. \& Lajtha, A. Handbook of neurochemistry and molecular neurobiology: Neurotransmitter systems. (Springer Science \& Business Media, 2008).

209 Siegel, G., Agranoff, B., Albers, R., Fisher, S. \& Uhler, M. Basic Neurochemistry: Molecular, Cellular and Medical Aspects, Sixth Edition. (Lippincott-Raven, 1999).

210 Rondou, P., Haegeman, G. \& Van Craenenbroeck, K. The dopamine D4 receptor: biochemical and signalling properties. Cellular and Molecular Life Sciences 67, 1971-1986, doi:10.1007/s00018-010-0293-y (2010).

211 Eglen, R. M. Muscarinic receptor subtypes in neuronal and non-neuronal cholinergic function. Autonomic and Autacoid Pharmacology 26, 219-233, doi:10.1111/j.1474-8673.2006.00368.x (2006).

212 Alberts, B., Johnson, A. \& Lewis, J. Molecular Biology of the Cell, Sixth Edition. (2015).

213 Nikolaev, V. O., Bünemann, M., Hein, L., Hannawacker, A. \& Lohse, M. J. Novel single chain cAMP sensors for receptor-induced signal propagation. Journal of Biological Chemistry 279, 37215-37218 (2004).

214 Depry, C., Allen, M. D. \& Zhang, J. Visualization of PKA activity in plasma membrane microdomains. Molecular BioSystems 7, 52-58 (2011).

215 Hackley, C. R., Mazzoni, E. O. \& Blau, J. cAMPr: A single-wavelength fluorescent sensor for cyclic AMP. Sci Signal 11 (2018).

216 Noam, Y., Bernard, C. \& Baram, T. Z. Towards an integrated view of HCN channel role in epilepsy. Current opinion in neurobiology 21, 873-879, doi:10.1016/j.conb.2011.06.013 (2011).

217 Zagotta, W. N. et al. Structural basis for modulation and agonist specificity of HCN pacemaker channels. Nature 425, 200-205 (2003).

218 Wainger, B. J., DeGennaro, M., Santoro, B., Siegelbaum, S. A. \& Tibbs, G. R. Molecular mechanism of cAMP modulation of HCN pacemaker channels. Nature 411, 805-810 (2001).

219 Zolles, G. et al. Pacemaking by HCN channels requires interaction with phosphoinositides. Neuron 52, 1027-1036 (2006).

220 Pian, P., Bucchi, A., Robinson, R. B. \& Siegelbaum, S. A. Regulation of gating and rundown of HCN hyperpolarization-activated channels by exogenous and endogenous PIP2. The Journal of general physiology 128, 593-604 (2006).

221 Jiang, Z.-G., Pessia, M. \& North, R. Dopamine and baclofen inhibit the hyperpolarizationactivated cation current in rat ventral tegmental neurones. The Journal of physiology 462, 753764 (1993). 
222 Alberi, S., Dreifuss, J. J. \& Raggenbass, M. The Oxytocin-induced Inward Current in Vagal Neurons of the Rat is Mediated by G Protein Activation but not by an Increase in the lntracellular Calcium Concentration. European Journal of Neuroscience 9, 2605-2612 (1997).

223 Connelly, W. M. et al. GABAB Receptors Regulate Extrasynaptic GABAA Receptors. J Neurosci 33, 3780-3785 (2013).

224 Mironov, S. L. et al. Imaging cytoplasmic cAMP in mouse brainstem neurons. BMC Neuroscience 10, 29, doi:10.1186/1471-2202-10-29 (2009).

225 Seamon, K. \& Daly, J. Forskolin: a unique diterpene activator of cyclic AMP-generating systems. Journal of cyclic nucleotide research 7, 201-224 (1981).

226 Harris, D., Asaad, M., Phillips, M., Goldenberg, H. \& Antonaccio, M. Inhibition of adenylate cyclase in human blood platelets by 9 -substituted adenine derivatives. Journal of cyclic nucleotide research 5, 125-134 (1979).

227 Xie, W. et al. Linderane suppresses hepatic gluconeogenesis by inhibiting the cAMP/PKA/CREB pathway through indirect activation of PDE 3 via ERK/STAT3. Frontiers in pharmacology 9, 476 (2018).

228 Hille, B. Ionic channels in excitable membranes. Current problems and biophysical approaches. Biophysical Journal 22, 283-294 (1978).

229 Mistrík, P. et al. The murine HCN3 gene encodes a hyperpolarization-activated cation channel with slow kinetics and unique response to cyclic nucleotides. Journal of Biological Chemistry 280, 27056-27061 (2005).

230 Ludwig, A. et al. Two pacemaker channels from human heart with profoundly different activation kinetics. The EMBO journal 18, 2323-2329 (1999).

231 Moroni, A. et al. Kinetic and ionic properties of the human HCN2 pacemaker channel. Pflĭgers Archiv 439, 618-626 (2000).

232 Harris, N. C. \& Constanti, A. Mechanism of block by ZD 7288 of the hyperpolarizationactivated inward rectifying current in guinea pig substantia nigra neurons in vitro. Journal of Neurophysiology 74, 2366-2378 (1995).

233 Savelieva, I. \& Camm, A. J. I f Inhibition with Ivabradine. Drug safety 31, 95-107 (2008).

234 Van Bogaert, P. P. \& Pittoors, F. Use-dependent blockade of cardiac pacemaker current (If) by cilobradine and zatebradine. European journal of pharmacology 478, 161-171 (2003).

235 Pape, H.-C. Queer current and pacemaker: the hyperpolarization-activated cation current in neurons. Annual review of physiology 58, 299-327 (1996).

236 McCormick, D. A. \& Bal, T. Sleep and arousal: thalamocortical mechanisms. Annual review of neuroscience 20, 185-215 (1997).

237 Steriade, M. \& Deschenes, M. The thalamus as a neuronal oscillator. Brain Research Reviews 8, 1-63 (1984).

238 Steriade, M., McCormick, D. A. \& Sejnowski, T. J. Thalamocortical oscillations in the sleeping and aroused brain. Science 262, 679-685 (1993).

239 Bal, T. \& McCormick, D. A. What stops synchronized thalamocortical oscillations? Neuron 17, 297-308 (1996).

240 Shibuya, M. Dopamine-sensitive adenylate cyclase activity in the striatum in Parkinson's disease. Journal of neural transmission 44, 287-295 (1979).

241 Herve, D. et al. Gaolf levels are regulated by receptor usage and control dopamine and adenosine action in the striatum. Journal of Neuroscience 21, 4390-4399 (2001).

242 Sancesario, G. et al. Down-regulation of nitrergic transmission in the rat striatum after chronic nigrostriatal deafferentation. European Journal of Neuroscience 20, 989-1000 (2004).

243 Giorgi, M. et al. Lowered cAMP and cGMP signalling in the brain during levodopa-induced dyskinesias in hemiparkinsonian rats: new aspects in the pathogenetic mechanisms. European Journal of Neuroscience 28, 941-950 (2008).

244 Reglődi, D., Lubics, A., Tamás, A., Szalontay, L. \& Lengvári, I. Pituitary adenylate cyclase activating polypeptide protects dopaminergic neurons and improves behavioral deficits in a rat model of Parkinson's disease. Behavioural brain research 151, 303-312 (2004).

245 Lamine-Ajili, A. et al. Effect of the pituitary adenylate cyclase-activating polypeptide on the autophagic activation observed in in vitro and in vivo models of Parkinson's disease. Biochimica et Biophysica Acta (BBA)-Molecular Basis of Disease 1862, 688-695 (2016). 
246 Čovičković-Šternić, N. et al. Cyclic nucleotides in cerebrospinal fluid of drug-free Parkinson patients. European neurology 27, 24-28 (1987).

247 Chan, C. S. et al. HCN channelopathy in external globus pallidus neurons in models of Parkinson's disease. Nature neuroscience 14, 85 (2011).

248 Good, C. H. et al. Impaired nigrostriatal function precedes behavioral deficits in a genetic mitochondrial model of Parkinson's disease. The FASEB journal 25, 1333-1344 (2011).

249 Masi, A., Narducci, R., Landucci, E., Moroni, F. \& Mannaioni, G. MPP+-dependent inhibition of Ih reduces spontaneous activity and enhances EPSP summation in nigral dopamine neurons. British journal of pharmacology 169, 130-142 (2013).

250 Kügler, S., Lingor, P., Schöll, U., Zolotukhin, S. \& Bähr, M. Differential transgene expression in brain cells in vivo and in vitro from AAV-2 vectors with small transcriptional control units. Virology 311, 89-95 (2003).

251 Brower, M., Grace, M., Kotz, C. M. \& Koya, V. Comparative analysis of growth characteristics of Sprague Dawley rats obtained from different sources. Laboratory animal research 31, 166173 (2015).

252 Kharbanda, S. et al. Role for Bcl-xL as an inhibitor of cytosolic cytochrome C accumulation in DNA damage-induced apoptosis. Proceedings of the National Academy of Sciences 94, 69396942 (1997).

253 Dehmelt, L. \& Halpain, S. The MAP2/Tau family of microtubule-associated proteins. Genome biology 6, 204-204, doi:10.1186/gb-2004-6-1-204 (2005).

254 Wood, J. G., Mirra, S. S., Pollock, N. J. \& Binder, L. I. Neurofibrillary tangles of Alzheimer disease share antigenic determinants with the axonal microtubule-associated protein tau (tau). Proceedings of the National Academy of Sciences 83, 4040-4043 (1986).

255 Akerboom, J. et al. Optimization of a GCaMP calcium indicator for neural activity imaging. Journal of neuroscience 32, 13819-13840 (2012).

256 Tian, L. et al. Imaging neural activity in worms, flies and mice with improved GCaMP calcium indicators. Nature methods 6, 875-881 (2009).

257 Shaner, N. C. et al. Improved monomeric red, orange and yellow fluorescent proteins derived from Discosoma sp. red fluorescent protein. Nature biotechnology 22, 1567-1572 (2004).

258 Hermens, W. T. et al. Purification of recombinant adeno-associated virus by iodixanol gradient ultracentrifugation allows rapid and reproducible preparation of vector stocks for gene transfer in the nervous system. Human gene therapy 10, 1885-1891 (1999).

259 Kügler, S., Hahnewald, R., Garrido, M. \& Reiss, J. Long-term rescue of a lethal inherited disease by adeno-associated virus-mediated gene transfer in a mouse model of Molybdenumcofactor deficiency. The American Journal of Human Genetics 80, 291-297 (2007).

260 Patel, T. P., Man, K., Firestein, B. L. \& Meaney, D. F. Automated quantification of neuronal networks and single-cell calcium dynamics using calcium imaging. Journal of neuroscience methods 243, 26-38 (2015).

261 Ferreira, T. \& Rasband, W. ImageJ user guide. ImageJ/Fiji 1, 155-161 (2012).

262 Phansalkar, N., More, S., Sabale, A. \& Joshi, M. in 2011 International Conference on Communications and Signal Processing. 218-220 (IEEE).

263 Legland, D., Arganda-Carreras, I. \& Andrey, P. MorphoLibJ: integrated library and plugins for mathematical morphology with ImageJ. Bioinformatics 32, 3532-3534 (2016).

264 Motulsky, H. Prism 4 statistics guide-statistical analyses for laboratory and clinical researchers. GraphPad Software Inc., San Diego, CA, 122-126 (2003).

265 Faul, F., Erdfelder, E., Buchner, A. \& Lang, A.-G. Statistical power analyses using G* Power 3.1: Tests for correlation and regression analyses. Behavior research methods 41, 1149-1160 (2009).

266 Healy-Stoffel, M., Ahmad, S. O., Stanford, J. A. \& Levant, B. A novel use of combined tyrosine hydroxylase and silver nucleolar staining to determine the effects of a unilateral intrastriatal 6hydroxydopamine lesion in the substantia nigra: a stereological study. Journal of neuroscience methods 210, 187-194 (2012).

267 Hosseini-Sharifabad, M. \& Nyengaard, J. R. Design-based estimation of neuronal number and individual neuronal volume in the rat hippocampus. Journal of neuroscience methods 162, 206214 (2007). 
268 Howard, C. V. et al. Measurement of total neuronal volume, surface area, and dendritic length following intracellular physiological recording. Neuroprotocols 2, 113-120 (1993).

269 Yuan, A., Rao, M. V. \& Nixon, R. A. (The Company of Biologists Ltd, 2012).

270 Caceres, A., Banker, G., Steward, O., Binder, L. \& Payne, M. MAP2 is localized to the dendrites of hippocampal neurons which develop in culture. Developmental Brain Research 13, 314-318 (1984).

271 Diógenes, M. J. et al. Extracellular alpha-synuclein oligomers modulate synaptic transmission and impair LTP via NMDA-receptor activation. Journal of Neuroscience 32, 11750-11762 (2012).

272 Borghi, R. et al. Full length $\alpha$-synuclein is present in cerebrospinal fluid from Parkinson's disease and normal subjects. Neuroscience letters 287, 65-67 (2000).

273 Volpicelli-Daley, L. A. et al. Exogenous $\alpha$-synuclein fibrils induce Lewy body pathology leading to synaptic dysfunction and neuron death. Neuron 72, 57-71 (2011).

274 Malik, J., Shevtsova, Z., Bähr, M. \& Kügler, S. Long-term in vivo inhibition of CNS neurodegeneration by Bcl-XL gene transfer. Molecular Therapy 11, 373-381 (2005).

275 Brewer, G. J. Isolation and culture of adult rat hippocampal neurons. Journal of Neuroscience Methods 71, 143-155 (1997).

276 Millet, L. J. \& Gillette, M. U. Over a century of neuron culture: from the hanging drop to microfluidic devices. The Yale journal of biology and medicine 85, 501 (2012).

277 Majumdar, D., Gao, Y., Li, D. \& Webb, D. J. Co-culture of neurons and glia in a novel microfluidic platform. Journal of neuroscience methods 196, 38-44 (2011).

278 Kügler, S. et al. Neuron-specific expression of therapeutic proteins: evaluation of different cellular promoters in recombinant adenoviral vectors. Molecular and Cellular Neuroscience 17, 78-96 (2001).

279 Pfrieger, F. W. \& Barres, B. A. Synaptic efficacy enhanced by glial cells in vitro. Science 277, 1684-1687 (1997).

280 Masquelier, T. \& Deco, G. Network bursting dynamics in excitatory cortical neuron cultures results from the combination of different adaptive mechanism. PloS one 8, e75824 (2013).

281 Van Pelt, J., Corner, M., Wolters, P., Rutten, W. \& Ramakers, G. Longterm stability and developmental changes in spontaneous network burst firing patterns in dissociated rat cerebral cortex cell cultures on multielectrode arrays. Neuroscience letters 361, 86-89 (2004).

282 Royo, N. C. et al. Specific AAV serotypes stably transduce primary hippocampal and cortical cultures with high efficiency and low toxicity. Brain research 1190, 15-22 (2008).

283 Palomeque, J. et al. Efficiency of eight different AAV serotypes in transducing rat myocardium in vivo. Gene therapy 14, 989-997 (2007).

284 Yamada, K. \& Iwatsubo, T. Extracellular $\alpha$-synuclein levels are regulated by neuronal activity. Molecular neurodegeneration 13, 1-8 (2018).

285 Kim, C. et al. Exposure to bacterial endotoxin generates a distinct strain of $\alpha$-synuclein fibril. Scientific reports 6, 1-12 (2016).

286 Polinski, N. K. et al. Best practices for generating and using alpha-synuclein pre-formed fibrils to model Parkinson's disease in rodents. Journal of Parkinson's disease 8, 303-322 (2018).

287 Šerý, O., Povová, J., Míšek, I., Pešák, L. \& Janout, V. Molecular mechanisms of neuropathological changes in Alzheimer's disease: a review. Folia Neuropathol 51, 1-9 (2013).

288 Chartier-Harlin, M.-C. et al. $\alpha$-synuclein locus duplication as a cause of familial Parkinson's disease. The Lancet 364, 1167-1169 (2004).

289 Cannon, J. et al. Neurosystems: brain rhythms and cognitive processing. European Journal of Neuroscience 39, 705-719 (2014).

290 Bhattacharyya, D. et al. Lipopolysaccharide from gut microbiota modulates $\alpha$-synuclein aggregation and alters its biological function. ACS chemical neuroscience 10, 2229-2236 (2019).

291 Sampson, T. R. et al. A gut bacterial amyloid promotes $\alpha$-synuclein aggregation and motor impairment in mice. Elife 9, e53111 (2020).

292 Chen, S. G. et al. Exposure to the functional bacterial amyloid protein curli enhances alphasynuclein aggregation in aged Fischer 344 rats and Caenorhabditis elegans. Scientific reports 6 , $1-10$ (2016). 
293 Angot, E., Steiner, J. A., Hansen, C., Li, J.-Y. \& Brundin, P. Are synucleinopathies prion-like disorders? The Lancet Neurology 9, 1128-1138 (2010).

294 Dickson, D. W. Neuropathology of non-Alzheimer degenerative disorders. International journal of clinical and experimental pathology 3, 1 (2010).

295 Bridi, J. C. \& Hirth, F. Mechanisms of $\alpha$-synuclein induced synaptopathy in Parkinson's disease. Frontiers in neuroscience 12, 80 (2018).

296 Dauer, W. \& Przedborski, S. Parkinson's disease: mechanisms and models. Neuron 39, 889-909 (2003).

297 Murray, A. J. \& Shewan, D. A. Epac mediates cyclic AMP-dependent axon growth, guidance and regeneration. Molecular and Cellular Neuroscience 38, 578-588 (2008).

298 Qiu, J. et al. Spinal axon regeneration induced by elevation of cyclic AMP. Neuron 34, 895-903 (2002).

299 Nirenberg, M. et al. Modulation of synapse formation by cyclic adenosine monophosphate. Science 222, 794-799 (1983).

300 Yamamoto, M., Urakubo, T., Tominaga-Yoshino, K. \& Ogura, A. Long-lasting synapse formation in cultured rat hippocampal neurons after repeated PKA activation. Brain research 1042, 6-16 (2005).

301 Tojima, T., Kobayashi, S. \& Ito, E. Dual role of cyclic AMP-dependent protein kinase in neuritogenesis and synaptogenesis during neuronal differentiation. Journal of neuroscience research 74, 829-837 (2003).

302 Petersen, K., Olesen, O. \& Mikkelsen, J. Developmental expression of $\alpha$-synuclein in rat hippocampus and cerebral cortex. Neuroscience 91, 651-659 (1999).

303 Bayer, T. A. et al. Neural expression profile of $\alpha$-synuclein in developing human cortex. Neuroreport 10, 2799-2803 (1999).

304 Kaneko, M. \& Takahashi, T. Presynaptic mechanism underlying cAMP-dependent synaptic potentiation. Journal of Neuroscience 24, 5202-5208 (2004).

305 Beyer, K. et al. New brain-specific beta-synuclein isoforms show expression ratio changes in Lewy body diseases. Neurogenetics 13, 61-72 (2012).

306 Beyer, K. et al. The decrease of $\beta$-synuclein in cortical brain areas defines a molecular subgroup of dementia with Lewy bodies. Brain 133, 3724-3733 (2010).

307 Evans, T., Kok, W. L., Cowan, K., Hefford, M. \& Anichtchik, O. Accumulation of betasynuclein in cortical neurons is associated with autophagy attenuation in the brains of dementia with Lewy body patients. Brain research 1681, 1-13 (2018).

308 Sharma, K. et al. Effect of Disease-Associated P123H and V70M Mutations on $\beta$-Synuclein Fibrillation. ACS Chemical Neuroscience 11, 2836-2848 (2020).

309 Janowska, M. K. \& Baum, J. The loss of inhibitory C-terminal conformations in disease associated P123H $\beta$-synuclein. Protein Science 25, 286-294 (2016).

310 Pissadaki, E. K. \& Bolam, J. P. The energy cost of action potential propagation in dopamine neurons: clues to susceptibility in Parkinson's disease. Frontiers in computational neuroscience 7, 13 (2013).

311 Matsuda, W. et al. Single nigrostriatal dopaminergic neurons form widely spread and highly dense axonal arborizations in the neostriatum. Journal of Neuroscience 29, 444-453 (2009).

312 Surmeier, D. J., Guzman, J. N. \& Sanchez-Padilla, J. Calcium, cellular aging, and selective neuronal vulnerability in Parkinson's disease. Cell calcium 47, 175-182 (2010).

313 Briel, R. et al. EEG findings in dementia with Lewy bodies and Alzheimer's disease. Journal of Neurology, Neurosurgery \& Psychiatry 66, 401-403 (1999).

314 Choi, S.-A., Evidente, V. G. H. \& Caviness, J. N. Comparing cerebral white matter lesion burdens between Parkinson's disease with and without dementia. Journal of movement disorders 3, 6 (2010).

315 Mukaetova-Ladinska, E. B. et al. Synaptic proteins and choline acetyltransferase loss in visual cortex in dementia with Lewy bodies. Journal of Neuropathology \& Experimental Neurology 72, 53-60 (2013).

316 Sabuncu, M. R. et al. The Dynamics of Cortical and Hippocampal Atrophy in Alzheimer Disease. Archives of Neurology 68, 1040-1048, doi:10.1001/archneurol.2011.167 (2011).

317 McDonald, C. R. et al. Regional rates of neocortical atrophy from normal aging to early Alzheimer disease. Neurology 73, 457-465, doi:10.1212/WNL.0b013e3181b16431 (2009). 
318 de la Monte, S. M. Quantitation of cerebral atrophy in preclinical and end-stage Alzheimer's disease. Annals of Neurology: Official Journal of the American Neurological Association and the Child Neurology Society 25, 450-459 (1989).

319 Claus, J. J. et al. Slowing on quantitative spectral EEG is a marker for rate of subsequent cognitive and functional decline in early Alzheimer disease. Alzheimer disease and associated disorders 12, 167-174 (1998).

320 Van der Hiele, K. et al. EEG correlates in the spectrum of cognitive decline. Clinical neurophysiology 118, 1931-1939 (2007).

321 Dissel, S. et al. Enhanced sleep reverses memory deficits and underlying pathology in drosophila models of Alzheimer's disease. Neurobiology of sleep and circadian rhythms 2, 1526 (2017). 


\section{Supplementary materials}

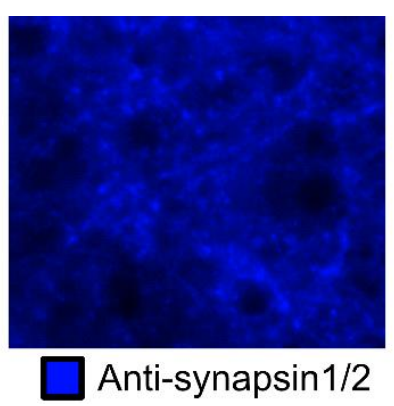

Supplementary Figure 1. Example stain using an antibody staining for synapse-specific protein synapsin1/2. Image of a neuronal culture immunocytochemically stained on DIV28 using anti-synapsin1/2 (blue, Synaptic Systems).

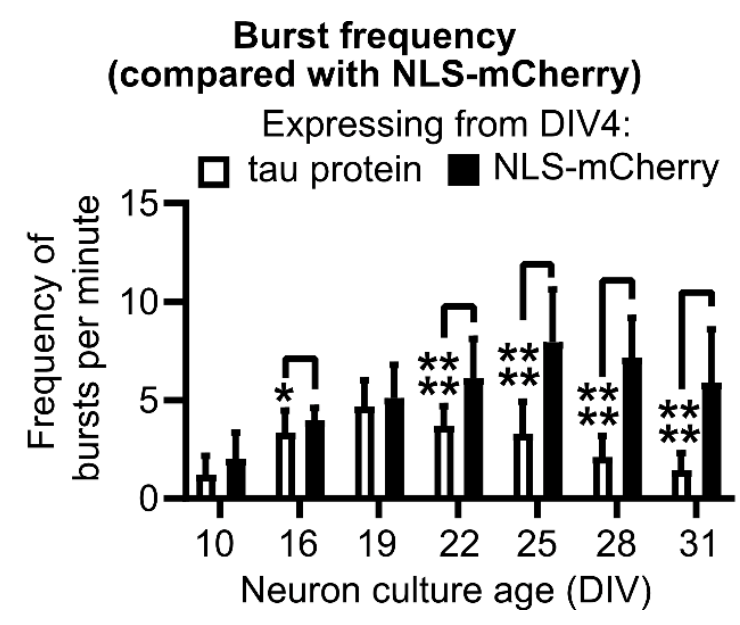

Supplementary Figure 2. Tau protein overexpression reduces the frequency of calcium bursting compared to expression of negative control NLS-mCherry.

Quantification of the frequency of calcium bursting in neurons overexpressing tau protein (white bars), which is compared to neurons expressing NLS-mCherry (black bars). NLS-mCherry = nuclear localization signal tagged mCherry. The frequency of bursts per minute \pm standard deviation is depicted. $*$ : $\mathrm{p}<0.05, * * * *: \mathrm{p}<0.0001$. Statistical analysis (used for all subfigures): two-tailed t-test with Welch's correction and a post-hoc power test. Statistical power: $1-\beta>57.6 \%$ (DIV16), $1-\beta>100 \%$ (DIV22, DIV25, DIV28, DIV31). Sample size: 18 independent cultures. 

A
Presynapses
B
Axons
C Dendrites
D Level of PKA phosphorylation

Expressing from DIV4: Expressing from DIV4: Expressing from DIV4: Expressing from DIV4:
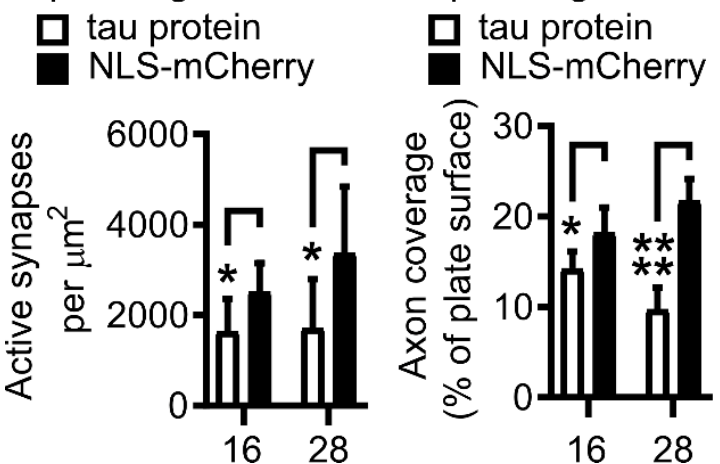

ㄴ tau protein

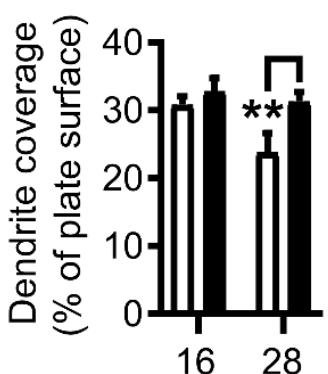

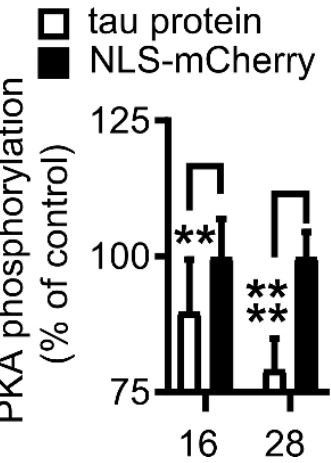

Supplementary Figure 3. Tau protein overexpression reduces the amount of active synapses, axons and dendrites, as well as the level of cAMP-dependent PKA phosphorylation in comparison to expression of NLS-mCherry.

(A-D) Quantification of the amount of pre-synapses (A), axons (B) and dendrites (C) as well as the level of PKA phosphorylation (D) in the presence of either tau protein overexpression (white bars) or NLS-mCherry expression (gray bars). The amount of pre-synapses per $\mathrm{mm}^{2} \pm$ standard deviation is depicted in (A), the percentage of plate surface covered by neurites \pm standard deviation is depicted in $(\mathbf{B}, \mathbf{C})$ and the level of PKA phosphorylation as $\%$ of control \pm standard deviation is depicted in (D). NLS-mCherry = nuclear localization signal tagged mCherry. $*: \mathrm{p}<0.05 * *: \mathrm{p}<0.01, * * * *: \mathrm{p}<0.0001$. Statistical analysis (used for all subfigures): two-tailed t-test with Welch's correction and a post-hoc power test. Statistical power: $1-\beta>75.2 \%$ for DIV16 and $1-\beta>73.5 \%$ for DIV28 (A), $1-\beta>76.9 \%$ for DIV16 and $1-\beta>100 \%$ for DIV28 (B), $1-\beta>100 \%$ (C), $1-\beta>77.6 \%$ for DIV16 and $1-\beta>100 \%$ for DIV28 (D). Sample size of independent cultures: 9 (A), 6 for DIV16 and 8 for DIV28 (B), 4 for DIV16 and 6 for DIV28 (C) and 11 for DIV16 and 16 for DIV28 (D). 


\section{List of abbreviations}

\begin{tabular}{|c|c|}
\hline$\alpha$ waves & Alpha waves \\
\hline$\alpha ; \alpha$-syn & $\alpha$-synuclein \\
\hline AAV & Adeno-associated virus \\
\hline $\mathrm{AC}$ & Adenyl cyclases \\
\hline $\mathrm{AD}$ & Alzheimer's disease \\
\hline ALS & Amylotrophic lateral sclerosis \\
\hline AMP & Adenosine monophosphate \\
\hline AMPA & $\alpha$-amino-3-hydroxy-5-methyl-4-isoxazolepropionic acid \\
\hline APP & Amyloid precursor protein \\
\hline ATP & Adenosine triphosphate \\
\hline $\mathrm{A} \beta$ & Amyloid beta \\
\hline$\beta$ waves & Beta waves \\
\hline$\beta$ & $\beta$-synuclein \\
\hline$\beta$-syn & $\beta$-synuclein \\
\hline BCA & Bicinchoninic acid \\
\hline Bcl-xL & B-cell lymphoma extra large \\
\hline BDNF & Brain-derived neurotrophic factor \\
\hline $\mathrm{bGH} \mathrm{pA}$ & Bovine growth hormone polyadenylation sequence \\
\hline BOLD & Blood oxygen level dependent \\
\hline BSA & Bovine serum albumin \\
\hline cAMP & Cyclic adenosine monophosphate \\
\hline cDNA & Complementary DNA \\
\hline cENOs & Cortical early network oscillations \\
\hline CFP & Cyan fluorescent protein \\
\hline $\mathrm{C}_{\mathrm{M}}$ & Molar concentration \\
\hline CREB & cAMP response element binding protein \\
\hline $\mathrm{CSF}$ & Cerebrospinal fluid \\
\hline Cy2 & Cyanine \\
\hline Cyclic AMP & Cyclic adenosine monophosphate \\
\hline$\delta$ waves & Delta waves \\
\hline DARPP-32 & Dopamine- and cAMP-regulated neuronal phosphoprotein \\
\hline$\overline{\mathrm{DDA}}$ & 2',3'-Dideoxyadenosine \\
\hline DIV & Day in vitro \\
\hline
\end{tabular}




\begin{tabular}{|c|c|}
\hline DLB & Dementia with Lewy bodies \\
\hline $\mathrm{DMN}$ & Default mode network \\
\hline DMSO & Dimethyl sulfoxide \\
\hline $\mathrm{dNTP}$ & Deoxynucleotide triphosphate \\
\hline DTT & Dithiothreitol \\
\hline E18 & Embryonic day 18 \\
\hline FCS & Fetal calf serum \\
\hline FFT & Fast Fourier transform method \\
\hline fMRI & Functional magnetic resonance imaging \\
\hline FRET & Förster resonance energy transfer \\
\hline GA & Glutaraldehyde \\
\hline GABA & Gamma-Aminobutyric acid \\
\hline GDP & Giant depolarizing potentials \\
\hline GFP & Green fluorescent protein \\
\hline HCN channels & Hyperpolarization-activated cyclic nucleotide gated channels \\
\hline HD & Huntington's disease \\
\hline HRP & Horse radish peroxidase \\
\hline hSyn & Human synapsin \\
\hline Int & Intronic sequence \\
\hline ITRs & Inverted terminal repeats \\
\hline LTP & Long-term potentiation \\
\hline $\mathrm{m}$ & Mass \\
\hline $\mathrm{M}$ & Molar mass \\
\hline M13 & M13 peptide sequence \\
\hline MAP kinase & Mitogen-activated protein kinase \\
\hline MAP2 & Microtubule-associated protein 2 \\
\hline$M A P T$ & Human tau protein (microtubule-associated protein tau) gene \\
\hline MCI & Mild cognitive impairment \\
\hline MPP+ & 1-methyl-4-phenylpyridinium \\
\hline "n.s" & Not significant \\
\hline NAC & Non amyloid-component \\
\hline NFL & Neurofilament L \\
\hline NFT & Neurofibrillary tangles \\
\hline NLS-mCherry & Nuclear localization signal tagged mCherry \\
\hline NMDA & N-methyl-D-aspartate \\
\hline$-\mathrm{OH}$ & Hydroxyl group \\
\hline
\end{tabular}




\begin{tabular}{|c|c|}
\hline $\mathrm{P} 123 \mathrm{H}$ & P123H $\beta$-synuclein mutant \\
\hline PBS & Phosphate-buffered saline \\
\hline PD & Parkinson's disease \\
\hline PD-D & Parkinson's disease dementia \\
\hline PDE & Phosphodiesterase \\
\hline PD-MCI & Parkinson's disease with mild cognitive impairment \\
\hline PFA & Paraformaldehyde \\
\hline PI & Protease inhibitor \\
\hline $\mathrm{PIP}_{2}$ & Phospatidylinositol-4,5-biphosphate \\
\hline PKA & Protein kinase A \\
\hline PKA-C & Catalytic subunit of PKA \\
\hline PKA-R & Regulatory subunit of PKA \\
\hline PVDF & Polyvinylidene fluoride \\
\hline qEEG & Quantitative encephalography \\
\hline qPCR & Quantitative PCR \\
\hline REM sleep & Rapid eye movement sleep \\
\hline SDS & Sodium dodecyl sulphate \\
\hline $\mathrm{SN}$ & Substantia nigra \\
\hline SNCA & Human $\alpha$-synuclein gene \\
\hline SNCG & $\gamma$-synuclein gene \\
\hline SV40 & Simian virus 40 polyadenylation site \\
\hline Sy-GCaMP & Synaptophysin-GCaMP \\
\hline$\tau$ & Microtubule-associated protein tau \\
\hline tau protein & Microtubule-associated protein tau \\
\hline TB & Transcription blocker \\
\hline $\mathrm{TE}$ & Tris-ethylenediaminetetraacetic acid \\
\hline$\theta$ waves & Theta waves \\
\hline TrkB & Tyrosine receptor kinase $\mathrm{B}$ \\
\hline V & Volume \\
\hline V70 & V70M $\beta$-synuclein mutant \\
\hline VAMP2 & Vesicle-associated membrane protein 2 \\
\hline WPRE & Woodchuck hepatitis virus post-transcriptional regulatory element \\
\hline YFP & Yellow fluorescent protein \\
\hline
\end{tabular}




\section{Acknowledgements}

I would firstly like to thank my supervisor, Dr. Sebastian Kügler, for guiding me through my doctoral project. I am especially appreciative of his constructive advice with regard to preparing this thesis, which was absolutely vital for its improvement to a sufficient level. In addition to this, I also believe that his assistance has been very beneficial in terms of helping me to advance my analytical as well as writing skills in a general sense.

I would furthermore like to thank Dr. Pretty Garg for collaborating with me in the creation of Figures 23 and 24, as well as Sonja Heyroth for collaborating with me in the creation of Figures 21 and Figure 22.

I would like to thank Monika Zebski for interesting conversations as well as helping me in a very large number of ways in performing my experiments.

I would also like to thank the DAAD for very generously providing me with funding during my doctoral thesis as well as opportunities for networking during my time here.

I would like to thank Prof. Dr. Mathias Bähr for allowing me to work in AG Kügler during my stay here, as well as his constructive advice during the seminars of the Department of Neurology.

I would like to thank Dr. Johan Tolö and Dr. Grit Taschenberger, who taught me a lot of the methods that were later expanded on to prepare the methodology of this project.

I would also like to thank Dr. Maryna Psol for allowing me to contribute to her project with regard to the impacts of the variants of $\beta$-synuclein.

I would additionally like to thank all other current and previous lab members for interesting discussions and help with my project, including Sofia Guerin, Claudia Fokken, Fabian Hobbie, Dr. Shi Cheng, Dr. Anupam Raina, Dr. Sameehan Mahajani and Dr. Julia Tereschenko.

I would also like to thank everyone of the Cellular and Molecular Physiology of the Brain program for the interesting retreats and student conferences, as well as the speakers of the program, Prof. Michael Hörner and Prof. Thomas Dresbach for their work in organizing the program.

I would like to also thank the members of my thesis committee for providing advice and constructive criticism during the thesis advisory committee meetings.

Finally, I would like to thank my family for supporting me during the course of my doctoral project. 